\title{
Sistemas de Equações de Reação-Difusão com Estrutura Anti-gradiente
}

\section{Carlos Henrique dos Santos}

Tese apresentada

ao

Instituto de Matemática e Estatística

da

Universidade de São Paulo

para

obtenção do grau de doutor

em

Matemática

Área de Concentração: Matemática Aplicada

Orientador: Prof. Dr. Luiz Augusto Fernandes de Oliveira

- São Paulo, novembro de 2005 - 


\section{Sistemas de Equações de Reação-Difusão com Estrutura Anti-gradiente}

Este exemplar corresponde à redação final da tese devidamente corrigida e defendida por Carlos Henrique dos Santos e aprovada pela comissão.

São Paulo, novembro de 2005.

Banca examinadora:

- Prof. Dr. Luiz Augusto Fernandes de Oliveira (orientador)

IME-USP

- Prof. Dr. Sérgio Muniz Oliva Filho

IME-USP

- Prof. Dr. Jair Silvério dos Santos FFCLRP-USP

- Prof. Dr. Hildebrando Munhoz Rodrigues

ICMC-USP

- Prof. Dr. Arnaldo Simal do Nascimento

UFSCar 
$\grave{A}$

Sra. Odete de Campos Alves, minha mãe,

exemplo de resistência, perseverança e humildade. 


\section{Agradecimentos}

Pelas normas vigentes, o número de páginas dedicadas aos agradecimentos não deve exceder o número daquelas relativas à tese. Não fosse isso, o registro dos agradecimentos a todas as pessoas a quem os devo constituiria uma volume muito maior do que o do trabalho. Entretanto, alguns são obrigatórios.

Inicialmente agradeço ao Prof. Dr. Luiz Augusto Fernandes de Oliveira pela orientação segura, pela paciência, compreensão e, sobretudo, pelo estímulo nos momentos adequados.

Gostaria de agradecer de modo especial aos amigos do Departamento de Matemática da UFPR pela inestimável ajuda e incentivo à conclusão deste trabalho. Em particular, agradeço aos profs. Elizabeth Karas, Edson Ribeiro Álvares e Sérgio Drumond Ventura que "carregaram o piano", aos profs. Alexandre Kirilov e João Batista Xavier pela retaguarda técnica, à profa. Florinda Miyaòka pelo apoio e encorajamento e ao prof. J. Yuan pelos "empurrões".

Agradeço à Simone Kugeratski Souza pela amizade e pelo apoio logístico e ao Alfredo Benedito Kugeratski Souza pelo suporte doméstico de informática. Agradeço ao prof. Marco Antônio Rodrigues Fernandes, amigo de longa data, pelas conversas estimulantes e pelas idéias peculiares da Física Médica. Especial agradecimento aos amigos José Carlos Corrêa Eidam e Raphaela, a ela pelos incentivos e a ele tanto pelas conversas ilustrativas e esclarecedoras sobre matemática quanto pelas dicas para diminuir os overfulls. Muito obrigado aos amigos do IME-USP, em especial, à Maria do Carmo, ao Marcone e à Rita de Cássia pela saudável convivência.

Finalmente, agradecimentos à minha família pelo apoio, carinho afetuoso e, principalmente, pela compreensão nas ausências inevitáveis. 


\section{Resumo}

Neste trabalho obtemos alguns critérios para decidir a instabilidade de equilíbrios não-constantes para algumas equações diferenciais parciais do tipo reação-difusão, expressas na forma de divergência, com coeficientes de difusão variáveis, a partir de algumas propriedades dos coeficientes e de certas propriedades geométricas dos domínios. Em seguida, adaptamos aos sistemas de equações de reação-difusão com acoplamento antigradiente resultados então obtidos. A razão para a escolha desse tipo de sistema é que, embora os sistemas anti-gradientes não preservem a ordem, eles podem ser decompostos parcialmente em dois sistemas do tipo gradiente acoplados de forma anti-simétrica. Essa particularidade permite dar aos sistemas com estrutura anti-gradiente um tratamento mais sistematizado com o uso das ferramentas de linearização usuais. 


\begin{abstract}
In this work we study some instability properties of non-constant equilibria for a class of reaction-diffusion partial differential equations with variable diffusive coefficients given in divergence form related to some properties of the coefficients of the equation and to some geometrical properties of the domain. The conclusions thus obtaind are extended to skew-gradient systems of equations due their particular property of being systems of gradient-type equations coupled in an anti-symmetric way. Though skew-gradient systems are not order preserving, the particular skew-gradient property of these systems allows the use of the usual tools of linearization to cope with this kind of problem.
\end{abstract}




\section{Sumário}

Introdução

1 A equação em meio heterogêneo 6

1.1 Formulação Geral . . . . . . . . . . . . . . . . . . . 6

1.2 Aspectos Geométricos do Problema . . . . . . . . . . . . . . . . . . . . . 11

1.3 Equações com Coeficientes Constantes . . . . . . . . . . . . . . . . . . . 14

1.4 Coeficientes Variáveis . . . . . . . . . . . . . . . . . . . . . . . . . 17

1.4.1 Caso Bidimensional . . . . . . . . . . . . . . . . . . 20

1.4 .2 O caso tridimensional . . . . . . . . . . . . . . . . 31

1.5 Coeficientes do Tipo $k(x) I_{N} \ldots \ldots \ldots \ldots$. . . . . . . . . 35

1.6 O Problema Escalar Heterogêneo . . . . . . . . . . . . . . . . . . 38

1.7 Domínios Convexos . . . . . . . . . . . . . . . . . . . . . 40

1.8 Domínios Simétricos . . . . . . . . . . . . . . . . . . . . 43

1.8.1 Domínios Anulares . . . . . . . . . . . . . . . . . . 46

2 Sistemas Unidimensionais $\quad 52$

2.1 Preliminares . . . . . . . . . . . . . . . . . . . . 52

2.2 Sistemas parciais . . . . . . . . . . . . . . . 58

2.3 Aplicações . . . . . . . . . . . . . . . . . . . . . . 64

2.4 As Equações de FitzHugh-Nagumo . . . . . . . . . . . . . . . . 76

2.4 .1 Estabilidade . . . . . . . . . . . . . . . . 77

3 Sistemas Anti-Gradientes $\quad 88$

3.1 Formulação Geral . . . . . . . . . . . . . . . . . . . . . . . . . . . . 89

3.2 Sistemas Parciais . . . . . . . . . . . . . . . . . . . . . 92 
3.3 O Sistema Original $\ldots \ldots \ldots \ldots \ldots$

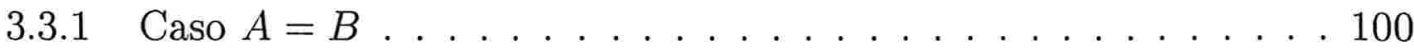

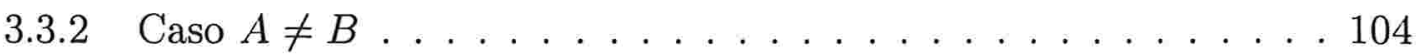

3.4 Uma Classe de Exemplos . . . . . . . . . . . . . . . . . 105

$\begin{array}{ll}\text { Conclusões } & 110\end{array}$

Referências Bibliográficas $\quad 113$ 


\section{Introdução}

O estudo dos sistemas de equações diferenciais parciais do tipo reação-difusão tem sido de grande interesse, tradicionalmente pelas bem conhecidas equações da Física-Matemática e, ultimamente, pela diversidade de aplicações na modelagem de fenômenos físicoquímicos, biológicos e de outras ciências aplicadas. Do ponto de vistá da Matemática a importância das equações e dos sistemas de equações diferenciais parciais se deve ao desenvolvimento de diversas áreas do próprio conhecimento matemático. Embora muitos desses sistemas tivessem origem na modelagem de fenômenos em áreas distintas do conhecimento científico, o interesse matemático por eles surgiu da riqueza de estruturas que as soluções podem apresentar. Para alguns dos sistemas já bem conhecidos ainda não há, atualmente, uma descrição completa de todos os aspectos envolvidos.

Com relação às aplicações práticas os equilíbrios representam situações limites de comportamentos a longo prazo dos fenômenos estudados. A razão principal para a sua descrição vem da necessidade de se fazer previsões e ou de se tomar decisões para longo prazo. Ainda sob esse aspecto, a estabilidade de soluções significa, por exemplo, a pouca influência que os erros experimentais podem ter nas conclusões ou descrições dos fenômenos modelados. Um outro aspecto importante em questão refere-se à validade dos modelos escolhidos e à coerência das soluções com os resultados experimentais.

Do ponto de vista matemático, o estudo dos equilíbrios e da estabilidade destes, originou problemas de grande importância e colaborou para o surgimento de novas áreas do conhecimento como, por exemplo, a topologia.

É interessante notar que diferentes fenômenos podem ser descritos por equações classificadas matematicamente como sendo da mesma natureza. A caracterização dos distintos comportamentos das soluções é um motivo de pesquisa e alguns aspectos em especial têm se mostrado influentes nessas variações tais como o papel dos coeficientes de 
difusividade, a influência da geometria do domínio e o papel do termo de não linearidade.

O papel que os coeficientes de difusividade desempenham na estabilidade ou na instabilidade dos equilíbrios tem despertado interesse desde 1975 quando Chafee, ver [8] mostrou que a equação $u_{t}=u_{x x}+f(u)$ definida num intervalo limitado I da reta com condições de Neumann homogêneas na fronteira não admite equilíbrio não-constante estável.

Casten e Holland, [7] e H. Matano, [33] mostraram que, quando $N \geq 1$, para as equações com coeficientes constantes do tipo $u_{t}=\Delta u+f(u)$ definidas em domínios convexos $\Omega \subset \mathbb{R}^{N}$, com condições de Neumann homogêneas na fronteira somente os equilíbrios constantes são estáveis. Matano mostrou que um resultado análogo ainda é válido para domínios não-convexos, mas com geometria especial como os obtidos por rotações de conjuntos convexos em torno de um eixo e os delimitados por esferas concêntricas. Neste mesmo trabalho Matano dá uma construção de domínio $\Omega$ nãoconvexo e de uma classe de funções $f$ para os quais o problema admite equilíbrio não constante estável.

Yanagida [42], Chipot e Hale [9], Fusco e Hale [19] consideraram equações unidimensionais com coeficientes variáveis do tipo $u_{t}=\left(a^{2}(x) u_{x}\right)_{x}+f(u), x \in(0,1), t>0$; tanto com condição de Neumann quanto com condição de Dirichlet homogêneas na fronteira mostrando que não existe equilíbrio não-constante estável quando $a^{\prime \prime}(x) \leq 0$ em $(0,1)$. Yanagida mostrou a existência de equilíbrios não-constantes estáveis quando $a^{\prime \prime}\left(x_{0}\right)>0$ para algum $x_{0} \in(0,1)$.

A existência de equilíbrios não-constantes estáveis para equações do tipo parabólico com coeficientes variáveis, independentemente da geometria do domínio, foi demonstrada por Nascimento [35] para uma classe de difusividades variáveis $k$ com termos não-lineares $f$ especificados.

O problema da instabilidade de equilíbrios para sistemas de equações diferenciais parciais com coeficientes constantes do tipo gradiente, definidos em domínios convexos, foi abordado por Kishimoto, [27], Kishimoto e Weinberger, [28] e Lopes, [31]. Em Yanagida [43] há uma abordagem dos sistemas com coeficientes constantes e estrutura anti-gradiente com a conclusão de que, em domínios convexos, não é possível, em geral, a existência de equilíbrios não-constantes estáveis.

Algumas propriedades dos equilíbrios das equações não são compartilhadas pelos 
equilíbrios dos sistemas de equações da mesma natureza. É sabido que, para as equações com coeficientes constantes definidas em domínios convexos, não é possível a existência de equilíbrios não-constantes estáveis, entretanto, existem sistemas de equações diferenciais parciais com coeficientes constantes com equilíbrios não-constantes estáveis como o caso do Exemplo 2.3.2. É de se esperar, portanto, que nos casos de sistemas com coeficientes variáveis também se possam obter equilíbrios não-constantes estáveis em analogia ao resultados apresentados em Yanagida [42], para $N=1$, e Nascimento [35], para $N \geq 2$.

Percebemos que há uma relação entre a geometria do domínio $\Omega$, o tipo de difusividade $D(x)$, a não-linearidade $f$ e a estabilidade de equilíbrios das equações dadas na forma de divergência e dos sistemas de equações dessa natureza. Em Yanagida [42] temos um resultado sobre a instabilidade de equilíbrios de uma equação definida num domínio convexo, um intervalo $\Omega$, com difusividade satisfazendo $a^{\prime \prime}(x) \leq 0$ em $\Omega$. Para que a equação admitisse equilíbrio não-constante estável, a hipótese admitida foi a existência de $x_{0} \in \Omega$ com $a^{\prime \prime}\left(x_{0}\right)>0$. Em Nascimento [35], para que haja estabilidade do equilíbrio, independentemente da geometria do domínio $\Omega$, a difusividade $k=k(x)$ deve satisfazer a condição $\Delta k(x)>0$ num subconjunto de $\Omega$. Portanto, como umạ generalização do resultado de Yanagida [42] para equações em dimensões mais altas, e também para os sistemas com difusividades variáveis em dimensão $N \geq 2$ definidos em domínios convexos, parece natural exigir que o Laplaciano da difusividade seja negativo ou nulo no domínio $\Omega$. Entretanto, como se verá neste trabalho, para as equações com coeficientes variáveis em dimensão $N \geq 2$, somente essa condição não é suficiente.

Este trabalho foi realizado com o propósito de estudar o problema da estabilidade e da instabilidade de equilíbrios para uma classe particular de equações diferenciais parciais do tipo reação-difusão com coeficientes de difusão variáveis, os chamados casos de difusividade não-homogênea. Para tanto, foram estabelecidas algumas condições sobre a matriz $D$ dos coeficientes de difusividades, sobre a geometria do domínio $\Omega$ e, eventualmente, sobre a função $f$ de modo a garantir resultados sobre a estabilidade ou a instabilidade dos equilíbrios, que generalizassem alguns dos resultados já bem conhecidos sobre as equações e os sistemas de coeficientes constantes. 
Mostramos, por exemplo, que, para problemas do tipo

$$
(P N E)\left\{\begin{array}{l}
u_{t}=\operatorname{div}(D(x) \nabla u)+f(u), x \in \Omega, t>0 \\
\frac{\partial u}{\partial \nu}=0, x \in \partial \Omega, t>0
\end{array}\right.
$$

definidos em domínios convexos $\Omega \subset \mathbb{R}^{2}$ particulares, com matriz de difusividade na forma diagonal $D(x)=\operatorname{diag}\left\{a^{2}, b^{2}\right\}$ satisfazendo $D \Delta a \leq 0$ e $\Delta b \leq 0$ em $\Omega, \frac{\partial a}{\partial \nu} \geq 0$ $\frac{\partial b}{\partial \nu} \geq 0$ em $\partial \Omega$, mais uma condição de não-positividade de uma forma quadrática em $\mathbb{R}^{2}$ definida a partir dos coeficientes de difusividade $a$ e $b$, só existem equilíbrios estáveis se forem constantes. Exibimos alguns exemplos de problemas com coeficientes variáveis definidos em domínios convexos para os quais vale o resultado.

Para os sistemas anti-gradientes da forma

$$
(P N S)\left\{\begin{aligned}
R u_{t} & =\operatorname{div}(A(x) \nabla u)+f(u, v), x \in \Omega, t>0 \\
S v_{t} & =\operatorname{div}(B(x) \nabla v)+g(u, v), x \in \Omega, t>0 \\
\frac{\partial u}{\partial \nu} & =0, x \in \partial \Omega, t>0 \\
\frac{\partial v}{\partial \nu} & =0, x \in \partial \Omega, t>0
\end{aligned}\right.
$$

definidos no domínio limitado convexo $\Omega \subset \mathbb{R}^{N}$, mostramos que, se as matrizes de difusividade $A, B$ forem do tipo diagonal e satisfizerem condições análogas à satisfeita pela matriz $D$ do problema (PNE) e, se as funções $f, g$ satisfizerem a condição $f_{v}^{T}(\phi, \psi) A+B^{T} g_{u}(\phi, \psi)=0$ em $\Omega$, então existem matrizes $R, S$ tais que um equilíbrio não-constante $(\phi, \psi)$ do sistema (PNS) correspondente, é instável.

O estudo da estabilidade ou instabilidade dos equilíbrios dos problemas em consideração será feito por meio da estabilidade linear seguindo Hale [22], Henry [23] ou Smoller [39]. O método da linearização para o estudo da estabilidade de um equilíbrio de um sistemas não-lineares de equações ordinárias do tipo $\frac{\mathrm{d} u}{\mathrm{~d} t}=f(u)$, onde $f: \mathbb{R}^{N} \longrightarrow \mathbb{R}^{N}$ é uma função diferenciável não-linear já é bem conhecido desde Poincaré. Consiste em decidir sobre a estabilidade do equilíbrio $u_{0} \in \mathbb{R}^{N}$ do sistema dado, por meio da estabilidade do equilíbrio 0 do sistema linear associado $\frac{\mathrm{d} u}{\mathrm{~d} t}=D f\left(u_{0}\right) u$. Vamos usar um resultado análogo para os sistemas de equações diferenciais parciais considerando o sistema dado como um sistema de equações diferenciais ordinárias do tipo $u_{t}=A u+f(u)$ num espaço de Banach adequado. O estudo da estabilidade de equilíbrios do sistema dado será feito considerando-se o espectro do operador linear $A$. No caso considerado neste trabalho, $A$ é um operador linear fortemente elíptico, auto-adjunto, com espectro 
discreto real composto por auto-valores. Uma das formas de se obter informações sobre o sinal do autovalor principal do operador linear correspondente é usar os teoremas de comparação, baseados no princípio do máximo como foi feito em Matano [33] ou em Kishimoto/Weinberger [28]. Entretanto, seguindo Jimbo e Morita [24], usaremos um método variacional para investigar o sinal do autovalor principal do operador correspondente ao sistema linearizado em torno do equilíbrio $\phi$ como apresentado em Gilbarg e Trudinger [21] ou em Smoller [39], por exemplo.

Para dar uma visão sobre a organização do trabalho citamos que no Capítulo 1, abordamos primordialmente o problema da estabilidade dos equilíbrios para as equações diferenciais parciais com coeficientes variáveis dadas na forma de divergência, com condições de Neumann homogêneas na fronteira. Nesse Capítulo provamos o Teorema 1.2.1 de caráter geométrico que é uma generalização de um resultado obtido em Matano [33] e que também aparece em Payne [36]. Esse Teorema é decisivo na conclusão sobre a instabilidade de equilíbrios não-constantes das equações diferenciais definidas em domínios limitados convexos; no Capítulo 2, usando os resultados obtidos no Capítulo 1 , tratamos o caso dos sistemas de equações com coeficientes variáveis, definidos em intervalos reais, como generalização natural dos resultados obtidos em Yanagida [42] para as equações escalares. No Capítulo 3, aplicamos alguns dos resultados dos Capítulos anteriores para estudar os sistemas anti-gradientes com coeficientes variáveis definidos em domínios convexos e limitados em dimensão $N \geq 2$. 


\section{Capítulo 1}

\section{A equação em meio heterogêneo}

Neste capítulo estudaremos o problema da estabilidade de equilíbrios da equação diferencial parcial de reação-difusão com coeficientes variáveis, dada na forma de divergência, definida em $\Omega \times(0, \infty)$ onde $\Omega$ é um domínio do espaço $\mathbb{R}^{N}$ com condições de Neumann homogêneas na fronteira $\partial \Omega$. Interessa-nos estudar a influência dos coeficientes de difusividade da equação, da geometria do domínio $\Omega$ sobre a estabilidade ou a instabilidade dos eventuais equilíbrios não-constantes. Para tanto vamos procurar estabelecer condições sobre os coeficientes de difusividade e sobre a geometria do domínio $\Omega$. O termo não-linear $f$ é decisivo na instabilidade dos equilíbrios no sentido de que se for uma função convexa então, independentemente da geometria do domínio, os equilíbrios serão instáveis. É claro que os zeros da função $f$ são os únicos equilíbrios constantes do problema. O estudo da estabilidade dos equilíbrios do problema dado será feito por meio da estabilidade do problema linearizado seguindo Hale [22], Henry [23] ou Smoller [39].

\subsection{Formulação Geral}

Consideremos o problema formulado num contexto mais geral de modo que possa ser tratado com as bem conhecidas ferramentas disponíveis conforme Hale [22] e Henry [23], por exemplo, mesmo que porteriormente se restrinja a alguns casos particulares. 
Seja o problema de valor inicial

$$
\left\{\begin{array}{l}
u_{t}=\operatorname{div}(A(x) \nabla u)+f(u), \quad x \in \Omega \quad t>0 \\
\frac{\partial u}{\partial \nu}=\langle A \nabla u, \vec{n}\rangle=0, \quad x \in \partial \Omega, \quad t>0,
\end{array}\right.
$$

onde $\Omega \subset \mathbb{R}^{N}$ é um domínio limitado, isto é, um subconjunto aberto, conexo e limitado com bordo $\partial \Omega$ classe $\mathscr{C}^{2}, f: \mathbb{R} \longrightarrow \mathbb{R}$ é uma função de classe $\mathscr{C}^{2}$ e a matriz $A(x)$ dada pela aplicação $A: \Omega \longrightarrow \mathbb{M}_{N}^{+}(\mathbb{R})$ de classe $\mathscr{C}^{2}$ que, a cada $x \in \Omega$, associa uma $N \times N$ matriz $A(x)=\left(a_{i j}(x)\right)_{1 \leq i, j \leq N}$ simétrica positiva-definida, isto é, para cada par $(i, j)$ as funções $a_{i j}: \Omega \rightarrow \mathbb{R}$ são de classe $\mathscr{C}^{2}$ e satisfazem as condições $a_{j i}(x)=a_{i j}(x), \forall x \in \Omega$ e para todo $i, j \in\{1, \ldots, N\}$. Além disso, existe $\alpha>0$ tal que, para todo $x \in \Omega$ e todo $\xi \in \mathbb{R}^{N}$, vale $\langle A(x) \xi, \xi\rangle \geq \alpha\|\xi\|^{2}$. O vetor $\vec{n}$ indica a direção normal exterior a $\Omega$ no ponto $x \in \partial \Omega$ e $\frac{\partial u}{\partial \nu}=\langle A \nabla u, \vec{n}\rangle$ indica a derivada co-normal de $u$ em $x$.

Admitiremos que a função $f$ satisfaça algumas condições de crescimento de modo que seja possível garantir suficiente dissipatividade como, por exemplo, quando existem constantes positivas $\beta, \gamma, \delta, \varepsilon$ satisfazendo

$$
u f(u) \leq-\varepsilon u^{2}+c_{\varepsilon}, \quad|f(u)| \leq \beta+\delta|u|^{\gamma}, \quad \forall u \in \mathbb{R}
$$

Definição 1.1.1 Uma solução clássica do problema (1.1) é uma função $u=u(x, t)$ $u \in \mathscr{C}^{0}(\bar{\Omega} \times[0, T]) \cap \mathscr{C}^{2}(\Omega \times(0, T))$ satisfazendo a equação cada $(x, t) \in \Omega \times(0, T)$ e as condições de fronteira para cada $x \in \partial \Omega$.

Mediante uma caracterização adequada dos espaços funcionais envolvidos, o problema dado pode ser representado por uma equação diferencial ordinária definida num espaço de Banach $\mathbf{X}$, da seguinte forma

$$
u_{t}=\mathbb{A} u+f^{e}(u)
$$

onde $\mathbb{A}: \mathscr{D}(\mathbb{A}) \subset \mathrm{X} \longrightarrow \mathrm{X}$ é um operador setorial para o qual os expoentes fracionários $\mathbb{A}^{\alpha}, 0<\alpha<1$, estão definidos nos espaços fracionários correspondentes, ver Henry [23]. No caso, $\mathbf{X}=\mathbf{L}^{2}(\Omega)$ e $\mathscr{D}(\mathbb{A})=\left\{u \in \mathrm{H}^{2}(\Omega) ; \frac{\partial u}{\partial \nu}=0, x \in \partial \Omega\right\}$ com $\mathbb{A}(w)=-\operatorname{div}(A(x) \nabla w)$. Pelas hipóteses admitidas sobre $f$ a função $f^{e}: X^{\alpha} \longrightarrow X$ é localmente Lipschitz contínua. 
Lembramos que um equilíbrio de (1.1) é uma função $\phi: \bar{\Omega} \longrightarrow \mathbb{R}$, pertencente a $\mathscr{C}^{1}(\bar{\Omega}) \cap \mathscr{C}^{2}(\Omega)$ satisfazendo o seguinte problema elíptico:

$$
\left\{\begin{array}{rll}
\operatorname{div}(A(x) \nabla \phi)+f(\phi) & =0, \quad x \in \Omega \\
\langle A(x) \nabla \phi, \vec{n}\rangle & =0, \quad x \in \partial \Omega
\end{array}\right.
$$

Um equilíbrio do problema (1.1) é um ponto crítico do funcional de energia $E: \mathbf{H}^{1}(\Omega) \longrightarrow$ $\mathbb{R}$ dado por

$$
E[u]=\int_{\Omega}\left\{\frac{1}{2}\langle A(x) \nabla u, \nabla u\rangle-F(u(x))\right\} \mathrm{d} x, \quad \forall u \in \mathbf{H}^{1}(\Omega),
$$

onde $F(u)=\int_{0}^{u} f(z) \mathrm{d} z$. Pelas hipóteses sobre a função $f$ o funcional de energia $E$ está bem definido e é de classe $\mathscr{C}^{1}$, pelo menos.

Dizemos que $\phi \in \mathbf{H}^{1}(\Omega)$ é um minimizador local do funcional (1.3) se existe $\delta>0$ tal que $E[\phi] \leq E[u]$, para todo $u \in \mathbf{H}^{1}(\Omega)$ satisfazendo $\|u-\phi\|_{\mathbf{H}^{1}(\Omega)}<\delta$. Notamos que se $u(x, t)$ é uma solução do sistema (1.1), então a função $[0, T) \ni t \longmapsto E(u(t))$ é diferenciável e vale

$$
\frac{\mathrm{d}}{\mathrm{d} t} E(u(t))=-\int_{\Omega}\left(u_{t}(x, t)\right)^{2} \mathrm{~d} x \leq 0 .
$$

Observamos que $\phi$ é um equilíbrio de (1.1) se, e somente se, é um ponto crítico do funcional (1.3) e o sistema (1.2) é chamado equação de Euler-Lagrange para esse funcional. A equação (1.1) descreve um fluxo gradiente do funcional $E$.

Definição 1.1.2 Um equilíbrio $\phi$ do problema (1.1) é dito estável (na topologia uniforme) se, para todo $\varepsilon>0$, existir $\delta>0$ tal que, para toda função $\psi=\psi(x)$ satisfazendo $\|\psi-\phi\|_{L^{\infty}(\Omega)}<\delta$, a solução $v=v(x, t)$ de $(1.1)$ com condição inicial $u(x, 0)=\psi(x)$ existe para todo $t \geq 0$ e satisfaz a condição

$$
\|v(x, t)-\phi(x)\|_{L^{\infty}(\Omega)}<\varepsilon, \quad \forall t>0 .
$$

Além disso, dizemos que o equilíbrio $\phi$ é assintoticamente estável se a solução $v(x, t)$ satisfizer a condição

$$
\lim _{t \rightarrow \infty}\|v(., t)-\phi\|_{L^{\infty}(\Omega)}=0 .
$$

A estabilidade de um equilíbrio $\phi$ do problema (1.1) está relacionada à estabilidade de 0 como equilíbrio de um problema linear associado conforme veremos a seguir. Seja 
$\phi=\phi(x)$ um equilíbrio do problema (1.1) e consideremos a linearização em torno de $\phi$ expressa por:

$$
\left\{\begin{aligned}
U_{t} & =\operatorname{div}(A(x) \nabla U)+f^{\prime}(\phi) U, \quad x \in \Omega, \quad t>0 \\
\frac{\partial U}{\partial \nu} & =\langle A(x) \nabla U, \vec{n}\rangle=0, \quad x \in \partial \Omega, \quad t>0 .
\end{aligned}\right.
$$

Observemos que os resultados estão sendo enunciados em espaços de energia mas, os cálculos, por uma razão de densidade, estão sendo realizadas com as funções suaves.

O estudo (local) da estabilidade dos equilíbrios do problema (1.1) via estabilidade do problema linear (1.4) correspondente será desenvolvido avaliando-se o sinal do autovalor principal, isto é, do menor autovalor do operador linear associado $\mathscr{L}: \mathbf{H}^{2}(\Omega) \longrightarrow \mathbf{L}^{2}(\Omega)$ dado por

$$
\mathscr{L}[v]=-\operatorname{div}(A(x) \nabla v)-f^{\prime}(\phi) v, \quad v \in \mathbf{H}^{2}(\Omega), \quad \frac{\partial v}{\partial \nu}=0 \text { em } \partial \Omega .
$$

Como o operador $\mathscr{L}$ é auto-adjunto, seu espectro é real e discreto, composto unicamente por uma seqüência $\left(\lambda_{n}\right)_{n \in \mathbb{N}}$ de autovalores satisfazendo $\lambda_{n} \rightarrow \infty$ quando $n \rightarrow \infty$. As correspondentes autofunções $\phi_{n}=\phi_{n}(x)$ formam um sistema ortonormal completo em $\mathbf{L}^{2}(\Omega)$. Designando por $\lambda_{1}$ o autovalor principal do operador $\mathscr{L}$, temos que se $\lambda_{1}<0$, então $\phi$ é instável como equilíbrio de (1.1), conforme Lions [30], Maginu [32], Matano [33], por exemplo.

Consideremos o problema de autovalores para o operador linearizado (1.5) expresso por:

$$
\left\{\begin{aligned}
\operatorname{div}(A(x) \nabla v)+f^{\prime}(\phi) v+\lambda v & =0, \quad x \in \Omega \\
\langle A(x) \nabla v, \vec{n}\rangle & =0, \quad x \in \partial \Omega .
\end{aligned}\right.
$$

Para avaliação do sinal do autovalor principal do operador (1.5) podemos considerar a equação do problema de autovalores (1.6). Multiplicando por $v \in \mathbf{H}^{2}(\Omega)$ e integrando sobre $\Omega$ obtemos

$$
\langle\mathscr{L}[v], v\rangle=\int_{\Omega}\left(-v \operatorname{div}(A(x) \nabla v)-f^{\prime}(\phi) v^{2}\right)=\lambda\|v\|_{\mathbf{L}^{2}}^{2} .
$$

Considerando que $\operatorname{div}(v A(x) \nabla v)=v \operatorname{div}(A(x) \nabla v)+\langle A(x) \nabla v, \nabla v\rangle$, podemos escrever

$$
\langle\mathscr{L}[v], v\rangle=\int_{\Omega}\left(-\operatorname{div}(v A(x) \nabla v)+\langle A(x) \nabla v, \nabla v\rangle-f^{\prime}(\phi) v^{2}\right) .
$$

Com as hipóteses sobre $\partial \Omega$ podemos aplicar o Teorema da Divergência, obtendo

$$
\langle\mathscr{L}[v], v\rangle=\int_{\Omega}\left(\langle A(x) \nabla v, \nabla v\rangle-f^{\prime}(\phi) v^{2}\right)-\int_{\partial \Omega} v \frac{\partial v}{\partial \nu} d \sigma .
$$


Da condição de fronteira $\frac{\partial v}{\partial \nu}=0$ segue que

$$
\langle\mathscr{L}[v], v\rangle=\int_{\Omega}\left(\langle A(x) \nabla v, \nabla v\rangle-f^{\prime}(\phi) v^{2}\right) .
$$

Conforme Smoller [39], os autovalores do operador $\mathscr{L}$ podem ser obtidos por meio do funcional $Q: \mathbf{H}^{1}(\Omega) \longrightarrow \mathbb{R}$ definido por

$$
Q[v]=\int_{\Omega}\left(\langle A \nabla v, \nabla v\rangle-f^{\prime}(\phi) v^{2}\right), \quad \forall v \in \mathbf{H}^{1}(\Omega)
$$

Notemos que se $v \in \mathscr{D}(\mathscr{L})$, isto é, se $v$ for uma função satisfazendo $\frac{\partial v}{\partial \nu}=0 \mathrm{em} \partial \Omega$, então

$$
Q[v]=\langle\mathscr{L}[v], v\rangle .
$$

Sabemos ainda que o ínfimo de $Q$ é atingido em uma função $v_{1}$ que é uma autofunção de $\mathscr{L}$ associada ao autovalor principal $\lambda_{1}=Q\left[v_{1}\right]$ satisfazendo $\frac{\partial v_{1}}{\partial \nu}=0$ em $\partial \Omega$.

Definição 1.1.3 Um equilíbrio $\phi$ do problema (1.1) é chamado linearmente estável quando existe $\delta>0$ tal que, para todo auto-valor $\lambda$ do operador linear $\mathscr{L}$, se tem $\operatorname{Re}(\lambda) \geq \delta$. Se existir um auto-valor $\lambda$ do operador $\mathscr{L}$ tal que $\operatorname{Re}(\lambda) \leq-\delta<0$, então diremos que $\phi$ será um equilíbrio linearmente instável do problema (1.1).

Notamos que se $\phi$ é linearmente estável (resp. instável) como equilíbrio de (1.1), então é estável (resp. instável) no sentido de Liapunov.

Definição 1.1.4 Dizemos que um equilíbrio $\phi$ do problema (1.1) é não-degenerado se o seu operador linear $\mathscr{L}$ associado é invertível.

Proposição 1.1.1 Um equilibrio não-degenerado $\phi$ de (1.1) é linearmente estável se, $e$ somente se, $Q[u] \geq 0, \forall u \in \mathbf{H}^{1}(\Omega)$.

Demonstração. Se o equilíbrio $\phi$ é linearmente estável, então o autovalor principal é caracterizado por $\lambda_{1}=\inf \left\{Q[u] ; u \in \mathbf{H}^{1}(\Omega) ;\|u\|_{\mathbf{H}^{1}}=1\right\}>0$, donde $Q[u] \geq 0 \forall u \in$ $\mathbf{H}^{1}(\Omega)$. Reciprocamente, se $\phi$ é equilíbrio não degenerado tal que $Q[u] \geq 0$ para todo $u \in \mathbf{H}^{1}(\Omega)$ então $\lambda_{1} \geq 0$. Se ocorresse $\lambda_{1}=0$, então $\lambda_{1}$ seria auto-valor do operador $\mathscr{L}$ associada à auto-função $u_{1}$ e o ínfimo de $Q$ seria atingido em $u_{1}$, isto é, $Q\left[u_{1}\right]=\lambda_{1}=0$. Neste caso, teríamos $\mathscr{L}\left(u_{1}\right)=0$, o que seria absurdo pela injetividade do operador. Logo, $\lambda_{1}>0$ e $\phi$ é linearmente estável. 
Proposição 1.1.2 Um equilíbrio linearmente instável de (1.1) não pode ser um mínimo local do funcional $E$.

Demonstração. Basta observar que se $\phi$ é linearmente instável, então $\lambda_{1} \leq-\delta<0$.

Observamos que um equilíbrio $\phi$ do sistema (1.1) é estável se, e somente se, o operador linear em torno de $\phi$ é injetivo. Um ponto crítico do funcional $E$ é equilíbrio estável de (1.1) se, e somente se, é não-degenerado.

Usaremos o seguinte resultado cuja demonstração pode ser encontrada em Fleming [15], em Matano [33] ou em Smoller [39].

Teorema 1.1.1 Se existe $\xi \in \mathbf{H}^{1}(\Omega)$ tal que $Q[\xi]<0$, então $\phi$ é instável como equilíbrio de (1.1).

Observação 1.1.1 Pela definição do funcional $Q$ dada em (1.9) temos que, se $f: \mathbb{R} \longrightarrow$ $\mathbb{R}$ é tal que $\int_{\Omega} f^{\prime}(\phi(x)) \mathrm{d} x \geq 0$, então $\phi$ é instável como equilíbrio de (1.1).

\subsection{Aspectos Geométricos do Problema}

Alguns resultados sobre a instabilidade de equilíbrios das equações dependem tanto de propriedades geométricas do domínio $\Omega$ como do comportamento geométrico do equilíbrio $\phi$ no bordo $\partial \Omega$. Vamos explorar alguns desses aspectos euUm resultado geral que podemos enunciar nessa direção é o seguinte teorema.

Teorema 1.2.1 Sejam $\Omega \subset \mathbb{R}^{N}$ um aberto limitado com bordo de classe $\mathscr{C}^{2}$ e $D=$ $\operatorname{diag}\left\{a_{1}^{2}(x), \ldots, a_{N}^{2}(x)\right\}$ uma matriz onde $a_{j}: \bar{\Omega} \rightarrow \mathbb{R}$ é diferenciável com $a_{j}(x)>0$ para todo $x \in \Omega, 1 \leq j \leq N$. Seja $\phi: \Omega \longrightarrow \mathbb{R}$ de classe $\mathscr{C}^{2}(\bar{\Omega})$ com derivada co-normal nula, em $\partial \Omega$. Se $\Omega$ é convexo, então, em $\partial \Omega$, vale a seguinte desigualdade

$$
\frac{\partial}{\partial \nu}(\langle D \nabla \phi, \nabla \phi\rangle)+2\left\langle\left(\sum_{j=1}^{N} a_{j}^{2} \phi_{x_{j}} D_{x_{j}}\right) \nabla \phi, \vec{n}\right\rangle-\left\langle\sum_{j=1}^{N} \phi_{x_{j}}^{2} D \nabla a_{j}^{2}, \vec{n}\right\rangle \leq 0 .
$$

Demonstração. Como $\partial \Omega$ é uma superfície regular de classe $\mathscr{C}^{2}$ podemos considerar localmente uma função $g: U \longrightarrow \mathbb{R}$ de classe $\mathscr{C}^{2}$ definida no aberto $U \subset \mathbb{R}^{N}$, com $\bar{\Omega} \cap U \neq \emptyset$ tal que $\partial \Omega \cap U=\{x \in U ; g(x)=0\}$ e $\nabla g(x) \neq 0, \forall x \in \partial \Omega \cap U$. 
Nessas condições, $\frac{\partial \phi}{\partial \nu}=\left\langle D \nabla \phi, \frac{\nabla g}{\|\nabla g\|}\right\rangle=0$ em $\partial \Omega \cap U$. Observamos que $\langle D \nabla \phi, \nabla g\rangle$ está definida para todo ponto $x \in \bar{\Omega} \cap U$. Seja $\xi: \bar{\Omega} \cap U \longrightarrow \mathbb{R}$ de classe $\mathscr{C}^{1}$, dada por $\langle D \nabla \phi(x), \nabla g(x)\rangle=\xi(x) g(x)$ para todo $x \in \bar{\Omega} \cap U$. Derivando essa expressão em relação a $x_{j}$, para $1 \leq j \leq N$, temos

$$
\left\langle D_{x_{j}} \nabla \phi, \nabla g\right\rangle+\left\langle D \nabla \phi_{x_{j}}, \nabla g\right\rangle+\left\langle D \nabla \phi, \nabla g_{x_{j}}\right\rangle=\xi_{x_{j}} g+\xi g_{x_{j}} .
$$

Multiplicando a igualdade membro a membro por $a_{j}^{2} \phi_{x_{j}}$ obtemos $\left\langle a_{j}^{2} \phi_{x_{j}} D_{x_{j}} \nabla \phi, \nabla g\right\rangle+\left\langle D a_{j}^{2} \phi_{x_{j}} \nabla \phi_{x_{j}}, \nabla g\right\rangle+a_{j}^{2} \phi_{x_{j}}\left\langle D \nabla \phi, \nabla g_{x_{j}}\right\rangle=g a_{j}^{2} \phi_{x_{j}} \xi_{x_{j}}+\xi a_{j}^{2} \phi_{x_{j}} g_{x_{j}}$.

Observamos que, para cada $j, 1 \leq j \leq N$, vale $a_{j}^{2} \phi_{x_{j}} \nabla \phi_{x_{j}}=\frac{1}{2} \nabla\left(a_{j}^{2} \phi_{x_{j}}\right)-\frac{1}{2} \phi_{x_{j}}^{2} \nabla a_{j}^{2}$, substituindo na expressão acima nos dá

$$
\begin{array}{r}
\left\langle a_{j}^{2} \phi_{x_{j}} D_{x_{j}} \nabla \phi, \nabla g\right\rangle+\frac{1}{2}\left\langle D \nabla\left(a_{j} \phi_{x_{j}}\right)^{2}, \nabla g\right\rangle-\frac{1}{2}\left\langle\phi_{x_{j}}^{2} D \nabla\left(a_{j}\right)^{2}, \nabla g\right\rangle+a_{j}^{2} \phi_{x_{j}}\left\langle D \nabla \phi, \nabla g_{x_{j}}\right\rangle \\
=g a_{j}^{2} \phi_{x_{j}} \xi_{x_{j}}+\xi a_{j}^{2} \phi_{x_{j}} g_{x_{j}} .
\end{array}
$$

Somando termo a termo a igualdade acima para $j, 1 \leq j \leq N$, obtemos

$$
\begin{aligned}
\left\langle\sum_{j=1}^{N} a_{j}^{2} \phi_{x_{j}} D_{x_{j}} \nabla \phi, \nabla g\right\rangle+ & \frac{1}{2}\left\langle\sum_{j=1}^{N} D \nabla\left(a_{j} \phi_{x_{j}}\right)^{2}, \nabla g\right\rangle-\frac{1}{2}\left\langle\sum_{j=1}^{N} \phi_{x_{j}}^{2} D \nabla\left(a_{j}\right)^{2}, \nabla g\right\rangle+ \\
& \sum_{j=1}^{N} a_{j}^{2} \phi_{x_{j}}\left\langle D \nabla \phi, \nabla g_{x_{j}}\right\rangle=g\langle D \nabla \phi, \nabla \xi\rangle+\xi\langle D \nabla \phi, \nabla g\rangle .
\end{aligned}
$$

Dividindo a igualdade, membro a membro, por $\|\nabla g\|$ e avaliando em $x \in \partial \Omega$ temos

$$
\begin{aligned}
\frac{\partial}{\partial \nu}(\langle D \nabla \phi, \nabla \phi\rangle)+2\left\langle\left(\sum_{j=1}^{N} a_{j}^{2} \phi_{x_{j}} D_{x_{j}}\right) \nabla \phi, \vec{n}\right\rangle & -\left\langle\sum_{j=1}^{N} \phi_{x_{j}}^{2} D \nabla a_{j}^{2}, \vec{n}\right\rangle \\
& =\frac{-2}{\|\nabla g\|}\langle G(x) \cdot D \nabla \phi, D \nabla \phi\rangle,
\end{aligned}
$$

onde estamos considerando $\sum_{j=1}^{N} a_{j}^{2} \phi_{x_{j}}^{2}=\langle D \nabla \phi, \nabla \phi\rangle$,

$$
\frac{\partial}{\partial \nu}(\langle D \nabla \phi, \nabla \phi\rangle)=\left\langle\sum_{j=1}^{N} D \nabla\left(a_{j} \phi_{x_{j}}\right)^{2}, \frac{\nabla g}{\|\nabla g\|}\right\rangle=\langle D \nabla(\langle D \nabla \phi, \nabla \phi\rangle), \vec{n}\rangle
$$

e $G(x) \cdot u=\sum_{i=1}^{N}\left(\sum_{j=1}^{N} \frac{\partial^{2} g(x)}{\partial x_{i} \partial x_{j}} u_{j}\right) e_{j}$, onde $e_{1}, \ldots, e_{N}$ são os vetores da base canônica do $\mathbb{R}^{N}$. Da convexidade de $\Omega$ decorre que a forma bilinear $G$, restrita ao espaço tangente 
a $\partial \Omega$ é positiva semi-definida. Da condição sobre $\phi$ no bordo, $\frac{\partial \phi}{\partial \nu}=\langle D \nabla \phi, \vec{n}\rangle=0$, temos que $D \nabla \phi$ pertence ao espaço tangente a $\partial \Omega$. Portanto, $\langle G \cdot(D \nabla \phi), D \nabla \phi\rangle \geq 0$ para todo $x \in \partial \Omega$, e, da expressão (1.10), segue que

$$
\frac{\partial}{\partial \nu}(\langle D \nabla \phi, \nabla \phi\rangle)+2\left\langle\left(\sum_{j=1}^{N} a_{j}^{2} \phi_{x_{j}} D_{x_{j}}\right) \nabla \phi, \vec{n}\right\rangle-\left\langle\sum_{j=1}^{N} \phi_{x_{j}}^{2} D \nabla a_{j}^{2}, \vec{n}\right\rangle \leq 0 .
$$

Em particular, para $N=2$, considerando $D(x, y)=\operatorname{diag}\left\{a^{2}(x, y), b^{2}(x, y)\right\}$ num domínio limitado $\Omega \subset \mathbb{R}^{2}$, para uma função $\phi: \bar{\Omega} \rightarrow \mathbb{R}$ verificando $\frac{\partial \phi}{\partial \nu}=0$ em $\partial \Omega$, vale a seguinte desigualdade em $\partial \Omega$

$$
\frac{\partial}{\partial \nu}(\langle D \nabla \phi, \nabla \phi\rangle)+2\left\langle\left(a^{2} \phi_{x} D_{x}+b^{2} \phi_{y} D_{y}\right) \nabla \phi, \vec{n}\right\rangle-\left\langle D\left(\phi_{x}^{2} \nabla a^{2}+\phi_{y}^{2} \nabla b^{2}\right), \vec{n}\right\rangle \leq 0 .
$$

Corolário 1.2.1 Suponha que $D=\operatorname{diag}\left\{d_{1}, \ldots, d_{N}\right\}$ seja uma matriz constante positiva, $\Omega \subset \mathbb{R}^{N}$ um aberto limitado com bordo de classe $\mathscr{C}^{2}$ e $\phi: \Omega \longrightarrow \mathbb{R}$ de classe $\mathscr{C}^{1}(\bar{\Omega})$ com derivada co-normal nula, em $\partial \Omega$. Se $\Omega$ é convexo, então

$$
\frac{\partial}{\partial \nu}(\langle D \nabla \phi, \nabla \phi\rangle) \leq 0 \quad \text { em } \quad \partial \Omega \text {. }
$$

Demonstração. Como $D$ é uma matriz com coeficientes constantes, a expressão (1.10) da demonstração do Teorema 1.2.1 se reduz a

$$
\frac{\partial}{\partial \nu}(\langle D \nabla \phi, \nabla \phi\rangle)=\frac{-2}{\|\nabla g\|}\langle G \cdot(D \nabla \phi), D \nabla \phi\rangle .
$$

Da convexidade de $\Omega$ decorre que a forma bilinear $G$, restrita ao espaço tangente a $\partial \Omega$ é positiva semi-definida. Da condição sobre $\phi$ no bordo, $\frac{\partial \phi}{\partial \nu}=\langle D \nabla \phi, \vec{n}\rangle=0$, temos que $D \nabla \phi$ pertence ao espaço tangente a $\partial \Omega$. Portanto, $\langle G(D \nabla \phi), D \nabla \phi\rangle \geq 0$ para todo $x \in \partial \Omega$, donde se conclui o resultado.

O Teorema 1.2.1 generaliza um resultado obtido por Matano, ver [33], e por Payne, ver [36], para um problema correspondente envolvendo ao operador Laplaciano referente à derivada normal da expressão $\|\nabla \phi\|^{2}$ em $\partial \Omega$, a saber, o Corolário seguinte. 
Corolário 1.2.2 Se $\Omega$ é convexo e se w é uma função com derivada normal nula em $\partial \Omega$, então $\frac{\partial}{\partial \vec{n}}\left(\|\nabla w\|^{2}\right) \leq 0$ em $\partial \Omega$.

Demonstração. Tome $D=I_{N}$, onde $I_{N}$ é a matriz identidade de ordem $N$, no argumento usado na demonstração do Teorema.

\subsection{Equações com Coeficientes Constantes}

Consideremos o problema da estabilidade de equilíbrios para uma equação com coeficientes constantes, onde é bem clara a influência de certas características geométricos sobre a estabilidade de equilíbrios não-constantes. Esperamos que a análise do problema nesta situação particular nos dê algumas indicações sobre os procedimentos que devem ser adotados para tratar o problema com coeficientes variáveis. Consideremos, portanto, o seguinte caso particular onde o sistema (1.1) é definido tendo por difusividade uma matriz diagonal $D=\operatorname{diag}\left\{d_{1}, \ldots, d_{N}\right\}$ onde as entradas $d_{j}, 1 \leq j \leq N$, são constantes positivas.

$$
\left\{\begin{array}{l}
u_{t}=\operatorname{div}(D \nabla u)+f(u), \quad x \in \Omega \quad t>0 \\
\frac{\partial u}{\partial \nu}=\langle D \nabla u, \vec{n}\rangle=0, \quad x \in \partial \Omega, \quad t \geq 0
\end{array}\right.
$$

Teorema 1.3.1 Seja $\phi$ um equilíbrio de (1.13). Se $\Omega$ é convexo e $\phi$ é não-constante, então $\phi$ é instável.

Demonstração. Sabemos que a instabilidade do equilíbrio da equação (1.13) é decidida avaliando o sinal do funcional $Q$. Vamos mostrar que existe uma função $w$ tal que $Q[w]<0$. Se $\phi$ é um equilíbrio não-constante de (1.13), podemos considerar, conforme Jimbo e Morita [24], para cada $j, j=1, \ldots, N$, o funcional $Q$ definido em (1.9) calculado na função $\sqrt{d_{j}} \phi_{x_{j}}$ :

$$
Q\left[\sqrt{d_{j}} \phi_{x_{j}}\right]=\int_{\Omega}\left(\left\langle D \nabla\left(\sqrt{d_{j}} \phi_{x_{j}}\right), \nabla\left(\sqrt{d_{j}} \phi_{x_{j}}\right)\right\rangle-f^{\prime}(\phi)\left(\sqrt{d_{j}} \phi_{x_{j}}\right)^{2}\right)
$$

isto é,

$$
Q\left[\sqrt{d_{j}} \phi_{x_{j}}\right]=\int_{\Omega} d_{j}\left(\left\langle D \nabla\left(\phi_{x_{j}}\right), \nabla\left(\phi_{x_{j}}\right)\right\rangle-f^{\prime}(\phi)\left(\phi_{x_{j}}\right)^{2}\right) .
$$

Da condição de equilíbrio temos $\operatorname{div}(D \nabla \phi)+f(\phi)=0$, donde, derivando em relação a $x_{j}$, obtemos $\operatorname{div}\left(D \nabla \phi_{x_{j}}\right)+f^{\prime}(\phi) \phi_{x_{j}}=0$. Multiplicando ambos os membros da igualdade 
por $\phi_{x_{j}}$ obtemos $\phi_{x_{j}} \operatorname{div}\left(D \nabla \phi_{x_{j}}\right)+f^{\prime}(\phi) \phi_{x_{j}}^{2}=0$, donde

$$
\operatorname{div}\left(\phi_{x_{j}} D \nabla \phi_{x_{j}}\right)-\left\langle D \nabla \phi_{x_{j}}, \nabla \phi_{x_{j}}\right\rangle+f^{\prime}(\phi) \phi_{x_{j}}^{2}=0 .
$$

Portanto,

$$
\left\langle D \nabla \phi_{x_{j}}, \nabla \phi_{x_{j}}\right\rangle-f^{\prime}(\phi) \phi_{x_{j}}^{2}=\frac{1}{2} \operatorname{div}\left(D \nabla \phi_{x_{j}}^{2}\right) .
$$

Substituindo na expressão do funcional podemos escrever

$$
Q\left[\sqrt{d_{j}} \phi_{j}\right]=\int_{\Omega}\left(\frac{d_{j}}{2} \operatorname{div}\left(D \nabla \phi_{x_{j}}^{2}\right)\right)=\frac{1}{2} \int_{\partial \Omega}\left(\left\langle D \nabla\left(d_{j} \phi_{x_{j}}^{2}\right), \vec{n}\right\rangle\right) \mathrm{d} \sigma \quad 1 \leq j \leq N .
$$

Somando a expressão acima para $1 \leq j \leq N$ obtemos

$$
\sum_{j=1}^{N} Q\left[\sqrt{d_{j}} \phi_{x_{j}}\right]=\frac{1}{2} \int_{\partial \Omega} \frac{\partial}{\partial \nu}(\langle D \nabla \phi, \nabla \phi\rangle) d \sigma .
$$

Pelo Corolário 1.2.1, temos

$$
\sum_{j=1}^{N} Q\left[\sqrt{d_{j}} \phi_{x_{j}}\right] \leq 0
$$

donde segue que, para algum $j, 1 \leq j \leq N$, tem-se $Q\left[\sqrt{d_{j}} \phi_{x_{j}}\right] \leq 0$, logo $\lambda_{1} \leq 0$. Se tivéssemos $\lambda_{1}=0$, então teríamos que a função $\sqrt{d_{j}} \phi_{x_{j}}$, com $\phi_{x_{j}} \not \equiv 0$, seria múltiplo da autofunção principal $u_{1}$ associada a $\lambda_{1}$. Então, existiria uma constante $m \neq 0$ tal que $\sqrt{d_{j}} \phi_{x_{j}}=m u_{1}$, onde a função $u_{1}$ pode ser escolhida de modo que $u_{1}(x)>0, \forall x \in \bar{\Omega}$. Da condição da fronteira tem-se $\langle D \nabla \phi, \vec{n}\rangle=0, \forall x \in \partial \Omega$. Como $\Omega$ é convexo e $\partial \Omega$ é suficientemente suave, existe $\bar{x} \in \partial \Omega$ tal que $\vec{n}=\vec{e}_{j}$ e, portanto, $\left\langle D \nabla \phi, \vec{e}_{j}\right\rangle=d_{j} \phi_{x_{j}}=0$. Como $d_{j}>0$, temos $\phi_{x_{j}}(\bar{x})=\frac{m}{\sqrt{d_{j}}} u_{1}(\bar{x})=0$, o que é absurdo. Portanto, $Q\left[\sqrt{d_{j}} \phi_{x_{j}}\right]<0$. Pelo Teorema 1.1.1, $\phi$ é instável como equilíbrio de (1.13).

Como conseqüência direta do Teorema 1.3.1 temos, como caso particular, o seguinte resultado bem conhecido obtido, independentemente, por Casten e Holland [7] e Matano [33].

Corolário 1.3.1 Sejam $\Omega \subset \mathbb{R}^{N}$ um aberto limitado com bordo $\partial \Omega$ suave e $\phi=\phi(x)$ um equilibrio não-constante do problema

$$
\left\{\begin{array}{l}
u_{t}=\Delta u+f(u), \quad x \in \Omega, \quad t>0 \\
\frac{\partial u}{\partial \vec{n}}=\langle\nabla u, \vec{n}\rangle=0, \quad x \in \partial \Omega, \quad t \geq 0 .
\end{array}\right.
$$

Se $\Omega$ é convexo, então $\phi$ é instável. 
Demonstração. Considerando a função $u=\phi_{x_{j}}$ na expressão (1.9) temos

$$
Q\left[\phi_{x_{j}}\right]=\frac{1}{2} \int_{\partial \Omega} \frac{\partial\left(\phi_{x_{j}}\right)^{2}}{\partial \vec{n}}, \quad 1 \leq j \leq N .
$$

Somando para $1 \leq j \leq N$ tem-se $\sum_{j=1}^{N} Q\left[\phi_{x_{j}}\right]=\frac{1}{2} \int_{\partial \Omega} \sum_{j=1}^{N} \frac{\partial\left(\phi_{x_{j}}\right)^{2}}{\partial \vec{n}} \mathrm{~d} \sigma$. Como a expressão do integrando acima é a derivada normal da função $\|\nabla \phi\|^{2}$, da convexidade do domínio e das condições de fronteira da função $\phi$, pelo Corolário 1.2.2 vem

$$
\sum_{j=1}^{N} Q\left[\phi_{x_{j}}\right]=\frac{1}{2} \int_{\partial \Omega} \frac{\partial}{\partial \vec{n}}\left(\|\nabla \phi\|^{2}\right) \mathrm{d} \sigma \leq 0 .
$$

Pelo Teorema 1.1.1 obtemos o resultado enunciado.

Para finalizar, vamos observar que o problema (1.13) com coeficientes constantes pode ser tratado diretamente com mudança de variáveis, De fato, no resultado a seguir mostramos como transformar (1.13) num problema do tipo considerado por Matano [33] e Casten e Holland [7]. Como a mudança de coordenadas só envolve homotetias, a propriedade da convexidade do domínio não se altera.

Lema 1.3.1 Existe um difeomorfismo $\Psi: \mathbb{R}^{N} \longrightarrow \mathbb{R}^{N}$ tal que $y=\Psi(x)$, em relação ao qual o problema (1.13) é dado por

$$
(P \tilde{N} C)\left\{\begin{aligned}
& \tilde{u}_{t}=\Delta \tilde{u}+f(\tilde{u}), \quad y \in \tilde{\Omega}, t>0 \\
& \frac{\partial \tilde{u}}{\partial \tilde{\tilde{n}}}=0, \quad y \in \partial \tilde{\Omega}, t>0 .
\end{aligned}\right.
$$

onde $\tilde{u}(y)=u(x) \operatorname{com} \tilde{\Omega}=\Psi(\Omega)$.

Demonstração. Como $D$ é uma matriz simétrica constante e positiva definida, então existe uma matriz ortogonal $P$ tal que $P^{T} D P=\operatorname{diag}\left\{\lambda_{1}, \ldots, \lambda_{N}\right\}$, onde $\lambda_{j}>0$ é autovalor de $D$. Seja $\Lambda=\operatorname{diag}\left\{\frac{1}{\sqrt{\lambda_{1}}}, \ldots, \frac{1}{\sqrt{\lambda_{N}}}\right\}$. Consideremos o difeomorfismo linear $\Psi: \mathbb{R}^{N} \longrightarrow \mathbb{R}^{N}$ dado por

$$
y=\Psi(x)=P \Lambda \cdot x, \quad \forall x \in \mathbb{R}^{N} .
$$

Nessas condições, observamos que, para cada $j$, vale $\frac{\partial u}{\partial x_{j}}=\sum_{k=1}^{N} \frac{\partial \tilde{u}}{\partial y_{k}} \frac{p_{k j}}{\sqrt{\lambda_{j}}}$. Portanto, para $1 \leq i, j \leq N$ temos

$$
\frac{\partial^{2} u}{\partial x_{i} \partial x_{j}}=\sum_{k, l=1}^{N} \frac{\partial^{2} \tilde{u}}{\partial y_{k} \partial y_{l}} \frac{p_{k i} p_{l j}}{\sqrt{\lambda_{i}} \sqrt{\lambda_{j}}} .
$$


Assim, a expressão da divergência na equação (1.13) se escreve como

$$
\begin{gathered}
\operatorname{div}(D \nabla u)=\sum_{i, j=1}^{N} a_{i j} \frac{\partial^{2} u}{\partial x_{i} \partial x_{j}}=\sum_{i, j=1}^{N} a_{i j}\left(\sum_{k, l=1}^{N} \frac{\partial^{2} \tilde{u}}{\partial y_{k} \partial y_{l}} \frac{p_{k i}}{\sqrt{\lambda_{i}}} \frac{p_{l j}}{\sqrt{\lambda_{j}}}\right) . \\
\operatorname{div}(D \nabla u)=\sum_{k, l=1}^{N}\left(\frac{p_{k i}}{\sqrt{\lambda_{i}}} a_{i j} \frac{p_{l j}}{\sqrt{\lambda_{j}}}\right) \frac{\partial^{2} \tilde{u}}{\partial y_{k} \partial y_{l}} .
\end{gathered}
$$

Como $(P \Lambda)^{T} D(P \Lambda)=\Lambda^{T} P^{T} D P \Lambda=I_{N}$ segue

$$
\operatorname{div}\left(D \nabla_{x} u\right)=\operatorname{div}\left(\nabla_{y} \tilde{u}\right)
$$

Considerando que a aplicação $\Psi$ é um difeomorfismo linear temos que se $\Omega$ é convexo, então $\Omega_{1}=\Psi(\Omega)$ também é convexo. Além disso, $\Psi$ leva o interior de $\Omega$ no interior de $\Omega_{1}$ e a fronteira de $\Omega$ na fronteira de $\Omega_{1}$. Vale também a seguinte condição $\nabla_{x} u=P \Lambda \nabla_{y} \tilde{u}$ donde

$$
\frac{\partial u}{\partial \nu}=\langle D \nabla u, \vec{n}\rangle=\left\langle D P \Lambda \nabla_{y} \tilde{u}, P \Lambda \overrightarrow{\tilde{n}}\right\rangle=\left\langle\nabla_{y} \tilde{u}, \overrightarrow{\tilde{n}}\right\rangle .
$$

Vale, portanto, $\frac{\partial u}{\partial \nu}(x)=\frac{\partial \tilde{u}}{\partial \tilde{\tilde{n}}}(y) \quad \forall x \in \partial \Omega$.

\subsection{Coeficientes Variáveis}

Nesta seção vamos abordar o problema da estabilidade de equilíbrios não-constantes para a equação (1.1) com coeficientes de difusão variáveis expressos na forma da matriz diagonal $D=D(x)$ definida num aberto $\Omega \subset \mathbb{R}^{N}$. Uma das razões para isso vem do fato que se $u=u(x, t)$ é uma solução do problema:

$$
(P N 1):\left\{\begin{aligned}
u_{t} & =\left(a^{2}(x) u_{x}\right)_{x}+f(u), \quad x \in(0,1) t>0 \\
u_{x}(0, t) & =u_{x}(1, t)=0, \quad t>0
\end{aligned}\right.
$$

então a função independente de $y$ definida por $w(x, y, t)=u(x, t)$ é uma solução do problema

$$
(P N 2):\left\{\begin{aligned}
w_{t} & =\left(a^{2}(x) w_{x}\right)_{x}+\left(b^{2}(y) w_{y}\right)_{y}+f(w), \quad(x, y) \in \Omega t>0 \\
w_{x}(0, y, t) & =w_{x}(1, y, t)=0,0<y<1, \quad t>0 \\
w_{y}(x, 0, t) & =w_{y}(x, 1, t)=0,0<x<1, \quad t>0
\end{aligned}\right.
$$


onde $\Omega=(0,1) \times(0,1)$. Além disso, para todo equilíbrio $\phi=\phi(x)$ de (PN1) a função independente de $y$ definida por $\Phi(x, y)=\phi(x)$ é um equilíbrio de (PN2). Se $\phi$ é instável como equilíbrio de (PN1), então $\Phi$ é instável como equilíbrio de (PN2). Portanto, algumas das conclusões obtidas para as equações unidimensionais traduzem-se para os problemas em dimensões mais altas como (PN2), por exemplo. Temos assim uma motivação para abordar os problemas análogos em dimensões maiores, tanto para os casos em que o domínio $\Omega$ é mais geral do que os blocos retangulares, quanto para os casos onde os coeficientes de difusividade não sejam constantes. Um outro motivo para considerar situações desse tipo vem do problema escalar tratado em Yanagida [42] e do resultado geométrico dado pelo Teorema 1.2.1.

Consideremos a equação

$$
\left\{\begin{array}{l}
u_{t}=\operatorname{div}(D(x) \nabla u)+f(u), \quad x \in \Omega, \quad t>0 \\
\frac{\partial u}{\partial \nu}=\langle D \nabla u, \vec{n}\rangle=0, \quad x \in \partial \Omega, \quad t \geq 0
\end{array}\right.
$$

onde $\Omega \subset \mathbb{R}^{N}$ um subconjunto aberto, conexo, limitado com bordo $\partial \Omega$ de classe $\mathscr{C}^{2}$, $f: \mathbb{R} \longrightarrow \mathbb{R}$ é uma função diferenciável e $D: \Omega \longrightarrow \mathbb{S}_{N}^{+}(\mathbb{R})$ é uma aplicação de classe $\mathscr{C}^{2}$ que a cada $x \in \Omega$ associa uma $N \times N$ matriz $D(x)=\operatorname{diag}\left\{d_{1}^{2}, \ldots, d_{N}^{2}\right\}$ positiva definida, isto é, para cada $j$, as funções $d_{j}: \Omega \rightarrow \mathbb{R}$ satisfazem $d_{j}(x) \geq d_{0}>0$, $\forall x \in \Omega, \vec{n}=\left(n_{1}, \ldots, n_{N}\right)$ indica o vetor normal exterior no ponto $x \in \partial \Omega$ e a expressão $\frac{\partial u}{\partial \nu}=\langle D \nabla u, \vec{n}\rangle=\sum_{j=1}^{N} d_{j}^{2} u_{j} n_{j}$ indica a derivada co-normal de $u$ em $x \in \partial \Omega$.

Um equilíbrio de (1.15) é uma função $\phi: \bar{\Omega} \longrightarrow \mathbb{R}$ de classe $\mathscr{C}^{2}(\Omega) \cap \mathscr{C}^{0}(\bar{\Omega})$ satisfazendo o seguinte problema elíptico:

$$
\left\{\begin{aligned}
\operatorname{div}(D(x) \nabla \phi)+f(\phi) & =0, \quad x \in \Omega \\
\langle D(x) \nabla \phi, \vec{n}\rangle & =0, \quad x \in \partial \Omega .
\end{aligned}\right.
$$

Vamos estudar a estabilidade dos equilíbrios do problema (1.15) via estabilidade do problema linear correspondente e, para tanto, precisamos avaliar o autovalor principal do operador linear $\mathscr{L}: \mathbf{H}^{2}(\Omega) \longrightarrow \mathrm{L}^{2}(\Omega)$ dado por

$$
\mathscr{L}[v]=-\operatorname{div}(D(x) \nabla v)-f^{\prime}(\phi) v
$$

onde $v \in \mathbf{H}^{2}(\Omega)$ e satisfaz $\frac{\partial v}{\partial \nu}=0$ em $\partial \Omega$. 
Consideremos o problema de auto-valores para o operador linearizado (1.17) expresso por:

$$
\left\{\begin{aligned}
\operatorname{div}(D(x) \nabla v)+f^{\prime}(\phi) v+\lambda v & =0, & x \in \Omega \\
\langle D(x) \nabla v, \vec{n}\rangle & =0, & x \in \partial \Omega .
\end{aligned}\right.
$$

Análogamente ao funcional definido em (1.9), consideremos o funcional $Q: \mathbf{H}^{1}(\Omega) \longrightarrow \mathbb{R}$ dado por

$$
Q[v]=\int_{\Omega}\left(\langle D \nabla v, \nabla v\rangle-f^{\prime}(\phi) v^{2}\right), \quad \forall v \in \mathbf{H}^{1}(\Omega) .
$$

Para provar que um equilíbrios $\phi$ é estável precisamos mostrar que todos os autovalores do operador (1.17) têm parte real positiva o que não é uma tarefa fácil em geral. Entretanto, para mostrar a instabilidade do equilíbrio $\phi$ basta mostrar que existe um autovalor com parte real negativa. Como nos casos que estamos abordando os autovalores do operador linear $\mathscr{L}$ são reais e obtidos por meio do funcional $Q$ vamos explorar algumas propriedades desse funcional e do fato de $\phi$ ser uma função definida em $\Omega$ com propriedades geométricas no bordo $\partial \Omega$. Analogamente ao que foi feito no caso das equações de coeficientes constantes temos em mente usar as conclusões geométricas do Teorema 1.2.1 para obter informações sobre o sinal do autovalor principal de $\mathscr{L}$. Se o problema (1.15) admite um equilíbrio não-constante $\phi=\phi(x)$ podemos considerar, para cada $j, j=1, \ldots, N$, o funcional $Q$ definido em (1.19) calculado na função $d_{j} \phi_{x_{j}}$

$$
Q\left[d_{j} \phi_{x_{j}}\right]=\int_{\Omega}\left[\left\langle D \nabla\left(d_{j} \phi_{x_{j}}\right), \nabla\left(d_{j} \phi_{x_{j}}\right)\right\rangle-f^{\prime}(\phi)\left(d_{j} \phi_{x_{j}}^{2}\right)\right) \mathrm{d} x, 1 \leq j \leq N .
$$

Pelos exemplos considerados por Yanagida [42], no caso $N=1$, um dos aspectos que interferem na estabilidade de equilíbrios é o sinal da segunda derivada dos coeficientes de difusão das equações estudadas pelo autor. Para as equações em dimensões $N \geq 2$ parece, então, ser razoável exigir uma condição análoga sobre a difusividade. Uma condição referente à segunda derivada diz respeito, por exemplo, ao Laplaciano das funções entradas da matriz $D$. Esta é uma motivação para investigar a influência do sinal do Laplaciano dos coeficientes da matriz $D$ sobre os equilíbrios do problema (1.15). Na tarefa de estimar o sinal do funcional $Q$, os cálculos efetuados acima mostram que há outras características que interferem na determinação da instabilidade de um equilíbrio não-constante do problema dado no caso de dimensão $N \geq 2$. Essas características referem-se às propriedades tanto geométricas quanto analíticas das funções de difusividade conforme veremos a seguir. 
Como o problema de estabelecer os domínios convexos para os quais seja válida uma generalização do resultado de Casten e Holland para equações com coeficientes variáveis no caso geral é algo complicado, vamos nos restringir a alguns modelos de domínios convexos quando $N=2$ e $N=3$ de modo a se ter uma noção razoável da situação e sem que isso comprometa a generalidade uma vez que, no caso geral, a situação é análoga.

\subsubsection{Caso Bidimensional}

Consideremos, portanto, o problema

$$
\left\{\begin{array}{l}
u_{t}=\left(a^{2}(x, y) u_{x}\right)_{x}+\left(b^{2}(x, y) u_{y}\right)_{y}+f(u), \quad(x, y) \in \Omega, \quad t>0 \\
\frac{\partial u}{\partial \nu}=a^{2} u_{x} n_{1}+b^{2} u_{y} n_{2}=0, \quad(x, y) \in \partial \Omega, \quad t \geq 0
\end{array}\right.
$$

onde $\Omega \subset \mathbb{R}^{2}$ um subconjunto aberto, conexo, limitado com bordo $\partial \Omega$ curva de classe $\mathscr{C}^{2}, f: \mathbb{R} \longrightarrow \mathbb{R}$ é uma função diferenciável e $D: \Omega \longrightarrow \mathbb{S}_{2}^{+}(\mathbb{R})$ é uma aplicação de classe $\mathscr{C}^{2}$ que a cada $x \in \Omega$ associa uma $2 \times 2$ matriz $D(x)=\operatorname{diag}\left\{a^{2}(x, y), b^{2}(x, y)\right\}$ positiva definida. As funções $a, b: \Omega \rightarrow \mathbb{R}$ satisfazem $a(x, y) \geq a_{0}>0, b(x, y) \geq b_{0}>0 \quad \forall x \in \Omega$. $\vec{n}=\left(n_{1}, n_{2}\right)$ indica o vetor normal exterior no ponto $(x, y) \in \partial \Omega$ e $a^{2} u_{x} n_{1}+b^{2} u_{y} n_{2}$ é a expressão da derivada co-normal de $u$ em $(x, y) \in \partial \Omega$. Como vimos no caso do estudo da estabilidade do equilíbrio das equações com coeficientes constantes, o sinal do autovalor principal foi decidido analisando o valor de uma expressão envolvendo o funcional $Q$. No caso das equações com coeficientes variáveis, usaremos um argumento semelhante. Para tanto estimamos o valor de uma expressão envolvendo $Q$ usando algumas funções particulares cujas propriedades são conhecidas.

Nessas condições, examinemos as expressões do funcional $Q$ avaliado nas funções $a \phi_{x}$ e $b \phi_{y}$, respectivamente. Para $u=a \phi_{x}$ temos

$$
Q\left[a \phi_{x}\right]=\int_{\Omega}\left(\left\langle D \nabla\left(a \phi_{x}\right), \nabla\left(a \phi_{x}\right)\right\rangle-f^{\prime}(\phi)\left(a \phi_{x}\right)^{2}\right) \mathrm{d} x
$$

Observamos que vale a seguinte propriedade $\operatorname{div}(u D \nabla u)=u \operatorname{div}(D \nabla u)-\langle D \nabla u, \nabla u\rangle$, donde $\langle D \nabla u, \nabla u\rangle=\frac{1}{2} \operatorname{div}\left(D \nabla(u)^{2}\right)-u \operatorname{div}(D \nabla u)$ e, com isso, podemos escrever o funcional como

$$
Q[u]=\frac{1}{2} \int_{\partial \Omega} \frac{\partial}{\partial \nu}\left(u^{2}\right) \mathrm{d} \sigma-\int_{\Omega} u\left(\operatorname{div}(D \nabla u)+f^{\prime}(\phi) u\right) .
$$


Assim, para $u=a \phi_{x}$ temos

$$
Q\left[a \phi_{x}\right]=\frac{1}{2} \int_{\partial \Omega} \frac{\partial}{\partial \nu}\left(a \phi_{x}\right)^{2}+\int_{\Omega}-\left(a \phi_{x}\right)\left(\operatorname{div}\left(D \nabla\left(a \phi_{x}\right)\right)+f^{\prime}(\phi)\left(a \phi_{x}\right)\right) \mathrm{d} x .
$$

Da condição de equilíbrio do problema dado vem $\operatorname{div}(D \nabla \phi)+f(\phi)=0$ que, derivada em relação a $x$ e multiplicada por $a^{2} \phi_{x}$, resulta

$$
a^{2} \phi_{x} \operatorname{div}\left(D_{x} \nabla \phi\right)+a \phi_{x}\left(a \operatorname{div}\left(D \nabla \phi_{x}\right)\right)+f^{\prime}(\phi)\left(a \phi_{x}\right)^{2}=0
$$

Pelas propriedades do divergente temos

$$
a^{2} \phi_{x} \operatorname{div}\left(D_{x} \nabla \phi\right)+a \phi_{x}\left(\operatorname{div}\left(a D \nabla \phi_{x}\right)-\left\langle D \nabla \phi_{x}, \nabla a\right\rangle\right)+f^{\prime}(\phi)\left(a \phi_{x}\right)^{2}=0
$$

donde vem

$\left.a^{2} \phi_{x} \operatorname{div}\left(D_{x} \nabla \phi\right)-a \phi_{x}\left\langle D \nabla \phi_{x}, \nabla a\right\rangle\right)+a \phi_{x}\left(\operatorname{div}\left(D \nabla\left(a \phi_{x}\right)-D \phi_{x} \nabla a\right)\right)+f^{\prime}(\phi)\left(a^{2} \phi_{x}\right)^{2}=0$.

Indicando por $\Sigma_{x}$ o integrando da segunda integral em (1.21), da equação acima podemos escrever

$$
\Sigma_{x}=a^{2} \phi_{x} \operatorname{div}\left(D_{x} \nabla \phi\right)-a \phi_{x}\left\langle D \nabla \phi_{x}, \nabla a\right\rangle-a \phi_{x} \operatorname{div}\left(\phi_{x} D \nabla a\right)
$$

Como

$$
\begin{aligned}
a^{2} \phi_{x} \operatorname{div}\left(D_{x} \nabla \phi\right) & =\operatorname{div}\left(a^{2} \phi_{x} D_{x} \nabla \phi\right)-\left\langle D_{x} \nabla \phi, \nabla\left(a^{2} \phi_{x}\right)\right\rangle, \\
a \phi_{x} \operatorname{div}\left(\phi_{x} D \nabla a\right) & =\operatorname{div}\left(a \phi_{x}^{2} D \nabla a\right)-\left\langle\phi_{x} D \nabla a, \nabla\left(a \phi_{x}\right)\right\rangle, \\
\left\langle\phi_{x} D \nabla a, \nabla\left(a \phi_{x}\right)\right\rangle & =\langle D \nabla a, \nabla a\rangle \phi_{x}^{2}+a \phi_{x}\left\langle D \nabla a, \nabla \phi_{x}\right\rangle
\end{aligned}
$$

substituindo na expressão (1.22), obtemos

$$
\Sigma_{x}=\operatorname{div}\left(a^{2} \phi_{x} D_{x} \nabla \phi\right)-\frac{1}{2} \operatorname{div}\left(\phi_{x}^{2} D \nabla a^{2}\right)-\left\langle\nabla \phi, D_{x} \nabla\left(a^{2} \phi_{x}\right)\right\rangle+\langle D \nabla a, \nabla a\rangle \phi_{x}^{2} .
$$

Assim, a segunda integral da expressão (1.21) se escreve como

$$
\begin{aligned}
\int_{\Omega} \Sigma_{x}=\int_{\partial \Omega}\left[\left\langle a^{2} \phi_{x} D_{x} \nabla \phi, \vec{n}\right\rangle-\frac{1}{2}\left\langle\phi_{x}^{2} D \nabla a^{2}, \vec{n}\right\rangle\right] \\
+\int_{\Omega}\left[\langle D \nabla a, \nabla a\rangle \phi_{x}^{2}-\left\langle D_{x} \nabla\left(a^{2} \phi_{x}\right), \nabla \phi\right\rangle\right] .
\end{aligned}
$$

Analogamente, a expressão do funcional $Q$ calculado na função $b \phi_{y}$ é

$$
Q\left[b \phi_{y}\right]=\frac{1}{2} \int_{\partial \Omega} \frac{\partial}{\partial \nu}\left(b \phi_{y}\right)^{2}+\int_{\Omega}-\left(b \phi_{y}\right)\left(\operatorname{div}\left(D \nabla\left(b \phi_{y}\right)\right)+f^{\prime}(\phi)\left(b \phi_{y}\right)\right) \mathrm{d} x .
$$


Para $\Sigma_{y}=-b \phi_{y}\left(\operatorname{div}\left(D \nabla\left(b \phi_{y}\right)\right)\right)+f^{\prime}(\phi)\left(b \phi_{y}\right)^{2}$ temos

$$
\Sigma_{y}=\operatorname{div}\left(b^{2} \phi_{y} D_{y} \nabla \phi\right)-\frac{1}{2} \operatorname{div}\left(\phi_{y}^{2} D \nabla b^{2}\right)-\left\langle\nabla \phi, D_{y} \nabla\left(b^{2} \phi_{y}\right)\right\rangle+\langle D \nabla b, \nabla b\rangle \phi_{y}^{2},
$$

donde

$$
\begin{aligned}
\int_{\Omega} \Sigma_{y}=\int_{\partial \Omega}\left[\left\langle b^{2} \phi_{y} D_{y} \nabla \phi, \vec{n}\right\rangle-\frac{1}{2}\left\langle\phi_{y}^{2} D \nabla b^{2}, \vec{n}\right\rangle\right] \\
+\int_{\Omega}\left[\langle D \nabla b, \nabla b\rangle \phi_{y}^{2}-\left\langle D_{y} \nabla\left(b^{2} \phi_{y}\right), \nabla \phi\right\rangle\right] .
\end{aligned}
$$

Representando por $\Sigma=Q\left[a \phi_{x}\right]+Q\left[b \phi_{y}\right]$, temos

$$
\Sigma=Q\left[a \phi_{x}\right]+Q\left[b \phi_{y}\right]=\frac{1}{2} \int_{\partial \Omega} \frac{\partial}{\partial \nu}(\langle D \nabla \phi, \nabla \phi\rangle)+\int_{\Omega}\left(\Sigma_{x}+\Sigma_{y}\right) .
$$

Usando as expressões (1.23) e (1.25), indicaremos $\Sigma$ por

$$
\begin{aligned}
\Sigma=\frac{1}{2} \int_{\partial \Omega}[ & \left.\frac{\partial}{\partial \nu}(\langle D \nabla \phi, \nabla \phi\rangle)+2\left\langle\left(a^{2} \phi_{x} D_{x}+b^{2} \phi_{y} D_{y}\right) \nabla \phi, \vec{n}\right\rangle-\left\langle D\left(\phi_{x}^{2} \nabla a^{2}+\phi_{y}^{2} \nabla b^{2}\right), \vec{n}\right\rangle\right] \\
& +\int_{\Omega}\left[\langle D \nabla a, \nabla a\rangle \phi_{x}^{2}+\langle D \nabla b, \nabla b\rangle \phi_{y}^{2}-\left\langle D_{x} \nabla\left(a^{2} \phi_{x}\right)+D_{y} \nabla\left(b^{2} \phi_{y}\right), \nabla \phi\right\rangle\right]
\end{aligned}
$$

No caso geral vale uma expressão correspondente, a saber,

$$
\begin{aligned}
\Sigma=\frac{1}{2} \int_{\partial \Omega}\left[\frac{\partial}{\partial \nu}(\langle D \nabla \phi, \nabla \phi\rangle)\right. & \left.+2\left\langle\left(\sum_{j} a_{j}^{2} \phi_{x_{j}} D_{x_{j}}\right) \nabla \phi, \vec{n}\right\rangle-\left\langle D\left(\sum_{j} \phi_{x_{j}}^{2} \nabla a_{j}^{2}\right), \vec{n}\right\rangle\right] \\
& +\int_{\Omega}\left[\sum_{j}\left\langle D \nabla a_{j}, \nabla a_{j}\right\rangle \phi_{x_{j}}^{2}-\left\langle\sum_{j} D_{x_{j}} \nabla\left(a_{j}^{2} \phi_{x_{j}}\right), \nabla \phi\right\rangle\right] .
\end{aligned}
$$

Quando $\Omega$ é um domínio limitado convexo e $\phi: \bar{\Omega} \rightarrow \mathbb{R}$ é tal que $\frac{\partial \phi}{\partial \nu}=0$ em $\partial \Omega$ então, pelo Teorema 1.2 .1 vale

$$
\frac{\partial}{\partial \nu}(\langle D \nabla \phi, \nabla \phi\rangle)+2\left\langle\left(\sum_{j} a_{j}^{2} \phi_{x_{j}} D_{x_{j}}\right) \nabla \phi, \vec{n}\right\rangle-\left\langle D\left(\sum_{j} \phi_{x_{j}}^{2} \nabla a_{j}^{2}\right), \vec{n}\right\rangle \leq 0 .
$$

Se $\Omega$ é um domínio limitado convexo com fronteira lisa por partes e $\phi: \bar{\Omega} \rightarrow \mathbb{R}$ tal que $\frac{\partial \phi}{\partial \nu}=0$ em $\partial \Omega$ então, pelo Teorema 1.2 .1 , temos

$$
\int_{\partial \Omega}\left[\frac{\partial}{\partial \nu}(\langle D \nabla \phi, \nabla \phi\rangle)+2\left\langle\left(\sum_{j} a_{j}^{2} \phi_{x_{j}} D_{x_{j}}\right) \nabla \phi, \vec{n}\right\rangle-\left\langle D\left(\sum_{j} \phi_{x_{j}}^{2} \nabla a_{j}^{2}\right), \vec{n}\right\rangle\right] \leq 0 .
$$


Portanto, quando o problema (1.20) for definido em domínios convexos do plano, a determinação do sinal da expressão $\Sigma=Q\left[a \phi_{x}\right]+Q b \phi_{y}$ reduzir-se-a à avaliação do sinal da integral sobre $\Omega$ dada em (1.26), para $N=2$. No caso geral a situação é análoga.

Indiquemos por $I$ a integral sobre $\Omega$ da expressão (1.26), dada por

$$
I=\int_{\Omega}\left[\langle D \nabla a, \nabla a\rangle \phi_{x}^{2}+\langle D \nabla b, \nabla b\rangle \phi_{y}^{2}-\left\langle D_{x} \nabla\left(a^{2} \phi_{x}\right)+D_{y} \nabla\left(b^{2} \phi_{y}\right), \nabla \phi\right\rangle\right] \mathrm{d} x .
$$

Vamos analisar as expressões no integrando de $I$ no sentido de estabelecer algumas relações entre os coeficientes de difusividade $a, b$ que nos permitam decidir em que condições se tem $I \leq 0$.

Para isso, observemos que

$$
\langle D \nabla a, \nabla a\rangle \phi_{x}^{2}+\langle D \nabla b, \nabla b\rangle \phi_{y}^{2}=a^{2} a_{x}^{2} \phi_{x}^{2}+b^{2} a_{y}^{2} \phi_{x}^{2}+a^{2} b_{x}^{2} \phi_{y}^{2}+b^{2} b_{y}^{2} \phi_{y}^{2} .
$$

Além disso, da expressão $\left\langle D_{x} \nabla\left(a^{2} \phi_{x}\right)+D_{y} \nabla\left(b^{2} \phi_{y}\right), \nabla \phi\right\rangle$, temos

$$
\left\langle D_{x} \nabla\left(a^{2} \phi_{x}\right), \nabla \phi\right\rangle=\left(a^{2}\right)_{x}\left(a^{2} \phi_{x}\right)_{x} \phi_{x}+\left(b^{2}\right)_{x}\left(a^{2} \phi_{x}\right)_{y} \phi_{y}
$$

onde cada parcela pode ser desenvolvida de modo que se tenha

$$
\left\{\begin{aligned}
\left(a^{2}\right)_{x}\left(a^{2} \phi_{x}\right)_{x} \phi_{x} & =a^{2} a_{x}^{2} \phi_{x}^{2}+\frac{\partial}{\partial x}\left(a\left(a \phi_{x}^{2}\right)^{2} a_{x}\right)-a \phi_{x}^{2} a^{2} a_{x x} \\
\left(b^{2}\right)_{x}\left(a^{2} \phi_{x}\right)_{y} \phi_{y} & =4 a a_{y} b b_{x} \phi_{x} \phi_{y}+\frac{\partial}{\partial x}\left(b\left(a \phi_{y}\right)^{2} b_{x}\right)-b \phi_{y}^{2} a^{2} b_{x x}-2 a a_{x} b b_{x} \phi_{y}^{2}-a^{2} b_{x}^{2} \phi_{y}^{2} .
\end{aligned}\right.
$$

Analogamente, para a parcela

$$
\left\langle D_{y} \nabla\left(b^{2} \phi_{y}\right), \nabla \phi\right\rangle=\left(a^{2}\right)_{y}\left(b^{2} \phi_{y}\right)_{x} \phi_{x}+\left(b^{2}\right)_{y}\left(b^{2} \phi_{y}\right)_{y} \phi_{y}
$$

temos,

$$
\left\{\begin{array}{l}
\left(b^{2}\right)_{y}\left(b^{2} \phi_{y}\right)_{y} \phi_{y}=b^{2} b_{y}^{2} \phi_{y}^{2}+\frac{\partial}{\partial y}\left(b\left(b \phi_{y}\right)^{2} b_{y}\right)-b \phi_{y}^{2} b^{2} b_{y y} \\
\left(a^{2}\right)_{y}\left(b^{2} \phi_{y}\right)_{x} \phi_{x}=4 a a_{y} b b_{x} \phi_{x} \phi_{y}+\frac{\partial}{\partial y}\left(a\left(b \phi_{y}\right)^{2} a_{y}\right)-a \phi_{y}^{2} b^{2} a_{y y}-2 a a_{y} b b_{y} \phi_{x}^{2}-b^{2} a_{y}^{2} \phi_{x}^{2} .
\end{array}\right.
$$

Substituindo essas expressões no integrando da expressão $I$ obtemos

$$
\begin{aligned}
I=-\int_{\Omega}\left[\operatorname{div}\left(a \phi_{x}^{2} D \nabla a\right)\right. & \left.+\operatorname{div}\left(b \phi_{y}^{2} D \nabla b\right)\right] \\
& +\int_{\Omega} a \phi_{x}^{2}\left(a^{2} a_{x x}+b^{2} a_{y y}\right)+b \phi_{y}^{2}\left(a^{2} b_{x x}+b^{2} b_{y y}\right) \\
& +2 \int_{\Omega}\left[\left(a a_{y} b b_{y}+b^{2} a_{y}^{2}\right) \phi_{x}^{2}-4 a a_{y} b b_{x} \phi_{x} \phi_{y}+\left(a a_{x} b b_{x}+a^{2} b_{x}^{2}\right) \phi_{y}^{2}\right] .
\end{aligned}
$$


Usando o Teorema da Divergência podemos escrever

$$
\begin{gathered}
I=-\int_{\partial \Omega}\left[a \phi_{x}^{2} \frac{\partial a}{\partial \nu}+b \phi_{y}^{2} \frac{\partial b}{\partial \nu}\right] \mathrm{d} \sigma+\int_{\Omega}\left[a \phi_{x}^{2}\left(a^{2} a_{x x}+b^{2} a_{y y}\right)+b \phi_{y}^{2}\left(a^{2} b_{x x}+b^{2} b_{y y}\right)\right] \mathrm{d} x \mathrm{~d} y \\
+2 \int_{\Omega}\left[\left(b^{2} a_{y}^{2}+a a_{y} b b_{y}\right) \phi_{x}^{2}-4 a a_{y} b b_{x} \phi_{x} \phi_{y}+\left(a^{2} b_{x}^{2}+a a_{x} b b_{x}\right) \phi_{y}^{2}\right] \mathrm{d} x \mathrm{~d} y
\end{gathered}
$$

onde $\frac{\partial a}{\partial \nu}=\langle D \nabla a, \vec{n}\rangle=a^{2} a_{x} n_{1}+b^{2} a_{y} n_{2}$ e $\frac{\partial b}{\partial \nu}=\langle D \nabla b, \vec{n}\rangle=a^{2} b_{x} n_{1}+b^{2} b_{y} n_{2}$.

Definindo as expressões $D \Delta a=a^{2} a_{x x}+b^{2} a_{y y}$ e $D \Delta b=a^{2} b_{x x}+b^{2} b_{y y}$ e a forma quadrática $\langle\mathbb{A} \xi, \xi\rangle, \xi \in \mathbb{R}^{2}$, com a matriz $\mathbb{A}=\mathbb{A}(x, y),(x, y) \in \Omega$, dada por

$$
\mathbb{A}=\left(\begin{array}{cc}
b^{2} a_{y}^{2}+a a_{y} b b_{y} & -2 a a_{y} b b_{x} \\
-2 a a_{y} b b_{x} & a^{2} b_{x}^{2}+a a_{x} b b_{x}
\end{array}\right)
$$

temos, em conseqüência, a seguinte expressão para a segunda integral $I$ de (1.26)

$$
I=-\int_{\partial \Omega}\left[a \phi_{x}^{2} \frac{\partial a}{\partial \nu}+b \phi_{y}^{2} \frac{\partial b}{\partial \nu}\right] \mathrm{d} \sigma+\int_{\Omega}\left[a \phi_{x}^{2} D \Delta a+b \phi_{y}^{2} D \Delta b\right]+2 \int_{\Omega}\langle\mathbb{A} \nabla \phi, \nabla \phi\rangle .
$$

Como a instabilidade do equilíbrio $\phi$ da equação (1.20) pode ser determinada pelo sinal da expressão $\Sigma$ os cálculos efetuados acima explicitaram as condições que permitem enunciar e provar o teorema seguinte.

Teorema 1.4.1 Seja $\phi$ um equilíbrio do problema (1.20) onde $\Omega \subset \mathbb{R}^{2}$ é um domínio limitado convexo com bordo $\partial \Omega$ uma curva de classe $\mathscr{C}^{2}$ e $D: \Omega \rightarrow \mathbb{M}_{2}(\mathbb{R})$ é uma matriz diagonal satisfazendo $D \Delta a \leq 0$ e $D \Delta b \leq 0 \mathrm{em} \Omega, \frac{\partial a}{\partial \nu} \geq 0$ e $\frac{\partial b}{\partial \nu} \geq 0$ em $\partial \Omega$ e a forma quadrática definida pela matriz $\mathbb{A}$, definida em (1.28), satisfaz $\langle\mathbb{A} \xi, \xi\rangle \leq 0$ em $\Omega$ para todo $\xi \in \mathbb{R}^{2}$. Nessas condições, se $\phi$ é um equilibrio não-constante do problema (1.20), então $\phi$ é instável.

Demonstração. Seja $\phi$ um equilíbrio não-constante do problema (1.20) e considere a expressão $\Sigma=Q\left[a \phi_{x}\right]+Q\left[b \phi_{y}\right]$ dada em (1.26). Da convexidade do domínio $\Omega$, pelo Teorema 1.2.1, fórmula (1.12), temos que a primeira integral da expressão (1.26) é menor que ou igual a zero. Quanto à segunda integral de (1.26), representada por $I$, os cálculos efetuados acima mostram que podemos indicar $I$ pela expressão (1.29). Pelas integrais envolvidas na expressão de $I$ e pelas hipóteses admitidas, temos que $I$ é menor que ou igual a zero, donde concluimos que

$$
\Sigma=Q\left[a \phi_{x}\right]+Q\left[b \phi_{y}\right] \leq 0 .
$$


Dessa desigualdade segue a instabilidade de $\phi$ como equilíbrio da equação dada.

A seguir temos vários exemplos de problemas com coeficientes de difusão variáveis, definidos em domínios convexos do plano para os quais valem as conclusões do Teorema 1.4.1.

Corolário 1.4.1 Consideremos um domínio limitado convexo $\Omega$ dado por

$$
\Omega=\left\{(x, y) \in \mathbb{R}^{2} ; f_{1}(x)<y<f_{2}(x) ; c<x<d\right\}
$$

onde $f_{1}, f_{2}:[c, d] \rightarrow \mathbb{R}$ são funções de classe $\mathscr{C}^{2}$. Seja $a:[c, d] \rightarrow \mathbb{R}$ de classe $\mathscr{C}^{2}$ tal que $a^{\prime \prime}(x) \leq 0, a^{\prime}(x) f_{2}^{\prime}(x) \leq 0$ e $a^{\prime}(x) f_{1}^{\prime}(x) \geq 0$ para todo $x$ em $[c, d]$. Nessas condições, se $\phi$ é um equilibrio não-constante do problema

$$
\left\{\begin{array}{l}
u_{t}=\left(a^{2}(x) u_{x}\right)_{x}+u_{y y}+f(u), \quad(x, y) \in \Omega, t>0 \\
\frac{\partial u}{\partial \nu}=a^{2}(x) u_{x} n_{1}+u_{y} n_{2}=0, \quad(x, y) \in \partial \Omega, t>0
\end{array}\right.
$$

então, $\phi$ é instável.

Demonstração. Admitamos que $\phi$ seja um equilíbrio não-constante do problema dado e consideremos a expressão $\Sigma=Q\left[a \phi_{x}\right]+Q\left[\phi_{y}\right]$ correspondente, conforme (1.26). Como $\Omega$ é convexo com a fronteira $\partial \Omega$ lisa por partes, pelo Teorema 1.2.1, fórmula (1.12), a primeira integral da expressão (1.26) é menor que ou igual a zero. Resta decidir o sinal da segunda integral. Para tanto vamos utilizar a expressão (1.29). Pelas hipóteses temos

$$
\left\{\begin{aligned}
D \Delta a & =a^{2} a_{x x}+b^{2} a_{y y}=a^{2} a_{x x} \text { em } \Omega \\
D \Delta b & =a^{2} b_{x x}+b^{2} b_{y y}=0 \text { em } \Omega, \\
\frac{\partial a}{\partial \nu} & =a^{2} a_{x} n_{1}+b^{2} a_{y} n_{2}=a^{2} a_{x} n_{1} \text { em } \partial \Omega \\
\frac{\partial b}{\partial \nu} & =a^{2} b_{x} n_{1}+b^{2} b_{y} n_{2}=0 \text { em } \partial \Omega
\end{aligned}\right.
$$

Como a matriz $\mathbb{A}$ é identicamente nula em $\Omega$ a forma quadrática definida é nula e, assim, a expressão (1.29) reduz-se a

$$
I=\int_{\Omega} a \phi_{x}^{2} a^{2} a_{x x} \mathrm{~d} x-\int_{\partial \Omega} a \phi_{x}^{2} a^{2} a_{x} n_{1} \mathrm{~d} \sigma .
$$

Por hipótese, $a_{x x} \leq 0$ em $\Omega$ donde vem $\int_{\Omega} a a_{x x}\left(a \phi_{x}\right)^{2} \mathrm{~d} x \leq 0$. Para concluir que $I \leq 0$ resta obter $\int_{\partial \Omega} a \phi_{x}^{2} a^{2} a_{x} n_{1} \mathrm{~d} \sigma \geq 0$. Para tanto, consideremos

$$
\int_{\partial \Omega} a \phi_{x}^{2} a^{2} a_{x} n_{1} \mathrm{~d} \sigma=\sum_{k=1}^{4} \int_{\partial \Omega_{k}} a \phi_{x}^{2} a^{2} a_{x} n_{1} \mathrm{~d} \sigma
$$


onde $\partial \Omega=\partial \Omega_{1} \cup \partial \Omega_{2} \cup \partial \Omega_{3} \cup \partial \Omega_{4}$.

Consideremos, por exemplo, o caso de um domínio $\Omega$ delimitado pelos gráficos das funções $f_{1}$ e $f_{2}$, conforme mostra a figura (1.1).

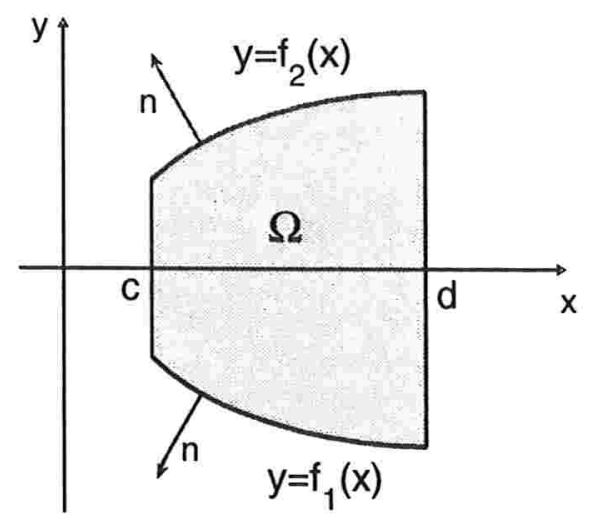

Figura 1.1: Domínio convexo delimitado por $f_{1}$ e $f_{2}$.

Sejam $\partial \Omega_{1}=\left\{(d, y) ; f_{1}(d) \leq y \leq f_{2}(d)\right\}$ com vetor normal exterior dado por $\vec{n}=(1,0)$ e $\partial \Omega_{3}=\left\{\left(c, f_{1}(c)+f_{2}(c)-y\right) ; f_{1}(c) \leq y \leq f_{2}(c)\right\}$ com vetor normal exterior $\vec{n}=(-1,0)$. Pelas condições de fronteira do problema temos $a^{2}(d) \phi_{x}(d, y)=0$ em $\partial \Omega_{1}$ $\mathrm{e}-a^{2}(c) \phi_{x}(c, y)=0$ em $\partial \Omega_{3}$ donde segue

$$
\int_{\partial \Omega_{1}} a \phi_{x}^{2} a^{2} a_{x} n_{1} \mathrm{~d} \sigma=\int_{\partial \Omega_{3}} a \phi_{x}^{2} a^{2} a_{x} n_{1} \mathrm{~d} \sigma=0 .
$$

Temos assim,

$$
\int_{\partial \Omega} a \phi_{x}^{2} a^{2} a_{x} n_{1} \mathrm{~d} \sigma=\int_{\partial \Omega_{2}} a \phi_{x}^{2} a^{2} a_{x} n_{1} \mathrm{~d} \sigma+\int_{\partial \Omega_{4}} a \phi_{x}^{2} a^{2} a_{x} n_{1} \mathrm{~d} \sigma .
$$

Considerando $\gamma:[c, d] \rightarrow \mathbb{R}^{2}$ dada por $\gamma(x)=\left(c+d-x, f_{2}(c+d-x)\right), c<x<d$, temos

$$
\partial \Omega_{2}=\left\{\left(c+d-x, f_{2}(c+d-x)\right) ; \quad c \leq x \leq d\right\}
$$

com vetor normal exterior dado por

$$
\vec{n}=\frac{1}{\sqrt{1+\left(f_{2}^{\prime}\right)^{2}}}\left(-f_{2}^{\prime}(c+d-x), 1\right), \quad c \leq x \leq d .
$$

Neste caso,

$$
\int_{\partial \Omega_{2}} a \phi_{x}^{2} a^{2} a_{x} n_{1} \mathrm{~d} \sigma=\int_{c}^{d} a(x) \phi_{x}^{2}(\gamma(x)) a^{2}(x)\left[-a^{\prime}(x) f_{2}^{\prime}(x)\right] \mathrm{d} x .
$$


Como $a^{\prime} f_{2}^{\prime} \leq 0$ em $(c, d)$ temos $\int_{\partial \Omega_{2}} a \phi_{x}^{2} a^{2} a_{x} n_{1} \mathrm{~d} \sigma \geq 0$.

Analogamente, para $\partial \Omega_{4}=\left\{\left(x, f_{1}(x)\right) ; \quad c \leq x \leq d\right\}$ com vetor normal exterior

$$
\vec{n}=\frac{1}{\sqrt{1+\left(f_{1}^{\prime}\right)^{2}}}\left(f_{1}^{\prime}(x),-1\right), \quad c \leq x \leq d,
$$

temos

$$
\int_{\partial \Omega_{4}} a \phi_{x}^{2} a^{2} a_{x} n_{1} \mathrm{~d} \sigma=\int_{c}^{d} a(x) \phi_{x}^{2}(\gamma(x)) a^{2}(x)\left[a^{\prime}(x) f_{1}^{\prime}(x)\right] \mathrm{d} x .
$$

Como $a^{\prime}(x) f_{1}^{\prime}(x) \geq 0$ vem que $\int_{\partial \Omega_{4}} a \phi_{x}^{2} a^{2} a_{x} n_{1} \mathrm{~d} \sigma \geq 0$.

Dos resultados acima temos que $\Sigma=Q\left[a \phi_{x}\right]+Q\left[\phi_{y}\right] \leq 0$, donde segue a instabilidade do equilíbrio $\phi$.

De maneira análoga podemos enunciar e provar o seguinte teorema.

Corolário 1.4.2 Consideremos um domínio limitado convexo $\Omega$ dado por

$$
\Omega=\left\{(x, y) \in \mathbb{R}^{2} ; g_{1}(y)<x<g_{2}(y) ; p<y<q\right\}
$$

onde $g_{1}, g_{2}:[p, q] \rightarrow \mathbb{R}$ são funções de classe $\mathscr{C}^{2}$. Seja $b:[p, q] \rightarrow \mathbb{R}$ de classe $\mathscr{C}^{2}$ tal que $b^{\prime \prime}(y) \leq 0, b^{\prime}(y) g_{2}^{\prime}(y) \leq 0$ e $b^{\prime}(y) g_{1}^{\prime}(y) \geq 0$ para todo $y$ em $[p, q]$. Nessas condições, se $\phi$ é um equilíbrio não-constante do problema

$$
(P N)=\left\{\begin{array}{l}
u_{t}=u_{x x}+\left(b^{2}(y) u_{y}\right)_{y}+f(u), \quad(x, y) \in \Omega, t>0 \\
\frac{\partial u}{\partial \nu}=u_{x} n_{1}+b^{2}(y) u_{y} n_{2}=0,(x, y) \in \partial \Omega, t>0
\end{array}\right.
$$

então, $\phi$ é instável.

Demonstração. A prova é análoga à do Corolário 1.4.1 considerando $\Sigma=Q\left[\phi_{x}\right]+$ $Q\left[b \phi_{y}\right]$ na expressão (1.26) e levando em consideração que, pelo Teorema 1.2 .1 , a primeira integral da expressão é menor que ou igual a zero e que a segunda integral reduz-se a

$$
I=\int_{\Omega} b \phi_{y}^{2} b^{2} b_{y y} \mathrm{~d} x-\int_{\partial \Omega} b \phi_{y}^{2} b^{2} b_{y} n_{2} \mathrm{~d} \sigma
$$


Corolário 1.4.3 Consideremos um domínio limitado convexo $\Omega$ dado por

$$
\Omega=\left\{(x, y) \in \mathbb{R}^{2} ; c<x<d, p<y<q\right\}
$$

Sejam $a:[c, d] \rightarrow \mathbb{R}$ e b $:[p, q] \rightarrow \mathbb{R}$ de classe $\mathscr{C}^{2}$ tais que $a^{\prime \prime}(x) \leq 0$ em $[c, d]$ e $b^{\prime \prime}(y) \leq 0$ em $[p, q]$. Nessas condições, se $\phi$ é um equilibrio não-constante do problema

$$
(P N)=\left\{\begin{array}{l}
u_{t}=\left(a^{2}(x) u_{x}\right)_{x}+\left(b^{2}(y) u_{y}\right)_{y}+f(u), \quad(x, y) \in \Omega, t>0 \\
\frac{\partial u}{\partial \nu}=a^{2}(x) u_{x} n_{1}+b^{2}(y) u_{y} n_{2}=0, \quad(x, y) \in \partial \Omega, t>0
\end{array}\right.
$$

então, $\phi$ é instável.

Demonstração. Consideremos a expressão $\Sigma=Q\left[a \phi_{x}\right]+Q\left[b \phi_{y}\right]$ dada em (1.26) onde

$$
I=\int_{\Omega}\left[a \phi_{x}^{2} a^{2} a_{x x}+b \phi_{y}^{2} b^{2} b_{y y}\right] \mathrm{d} x-\int_{\partial \Omega}\left[a \phi_{x}^{2} a^{2} a_{x} n_{1}+b \phi_{y}^{2} b^{2} b_{y} n_{2}\right] \mathrm{d} \sigma .
$$

Como o domínio $\Omega$ é um convexo com bordo liso por partes sabemos que a integral sobre $\partial \Omega$ é menor que ou igual a zero. Como $\phi$ é um equilíbrio do problema, notamos que a integral sobre $\partial \Omega$ da expressão $I$ acima é nula. Além disso, por hipótese também temos $a_{x x} \leq 0$ e $b_{y y} \leq 0$, donde $I \leq 0$ e, conseqüentemente, $\Sigma \leq 0$ donde segue a instabilidade do equilíbrio $\phi$.

Observação 1.4.1 Analisemos as condições do Corolário 1.4.1 onde o domínio convexo $\Omega$ é delimitado pelas retas verticais $x=c, x=d$ e pelos gráficos das funções $f_{1}, f_{2}$ : $[c, d] \rightarrow \mathbb{R}$, de classe $\mathscr{C}^{2}$, onde a difusividade $a:[c, d] \rightarrow \mathbb{R}$ é de classe $\mathscr{C}^{2}$ satisfazendo $a^{\prime \prime}(x) \leq 0, a^{\prime}(x) f_{2}^{\prime}(x) \leq 0$ e $a^{\prime}(x) f_{1}^{\prime}(x) \geq 0$ para todo $x$ em $[c, d]$.

Pela convexidade de $\Omega$ temos que $f_{2}^{\prime \prime} \leq 0$ e que $f_{1}^{\prime \prime} \geq 0$ em $[c, d]$. No caso em que $f_{1}$ e $f_{2}$ são monótonas temos quatro possibilidades distintas.

(a). $f_{1}^{\prime} \leq 0$ e $f_{2}^{\prime} \geq 0$, como na figura (1.1). Nesse caso, pela hipóteses $a^{\prime} f_{2}^{\prime} \leq 0$ e $a^{\prime} f_{1}^{\prime} \geq 0$ temos que $a^{\prime} \leq 0$. Como $a^{\prime \prime} \leq 0$ vem que $a$ deve ser uma função não-crescente com concavidade voltada para baixo. Como exemplos de funções com essas características temos $a(x)=m x+n, m<0$, satisfazendo $m d+n>0$ e $a(x)=M+\log (1+d-x)$ $\operatorname{com} M>0$.

(b). $f_{1}^{\prime} \geq 0$ e $f_{2}^{\prime} \leq 0$ Nesse caso, pela hipóteses $a^{\prime} f_{2}^{\prime} \leq 0$ e $a^{\prime} f_{1}^{\prime} \geq 0$ temos que $a^{\prime} \geq 0$. De $a^{\prime \prime} \leq 0$, vem que $a$ deve ser uma função não-decrescente com concavidade voltada 
para baixo. Como exemplos temos $a(x)=m x+n, m>0$, satisfazendo $m c+n>0$ e $a(x)=M+\log (1+x-c) \operatorname{com} M>0$.

(c). $f_{1}^{\prime} \geq 0$ e $f_{2}^{\prime} \geq 0$ Nesse caso, pela hipóteses $a^{\prime} f_{2}^{\prime} \leq 0$ e $a^{\prime} f_{1}^{\prime} \geq 0$ temos que $a^{\prime} \leq 0$ e que $a^{\prime} \geq 0$. Portanto, $a^{\prime}=0$ e $a$ é constante.

(d). Analogamente ao caso (c), se $f_{1}^{\prime} \leq 0$ e $f_{2}^{\prime} \leq 0$, temos que $a$ é constante.

Por outro lado, no caso em que $f_{1}$ é constante e $f_{2}$ não é monótona pelas hipóteses do Corolário 1.4.1 devemos ter $a^{\prime} \leq 0$ onde $f_{2}^{\prime} \geq 0$ e $a^{\prime} \geq 0$ onde $f_{2}^{\prime} \leq 0$. Nesse caso, a condição $a^{\prime \prime} \leq 0$ indica que $a$ deve ser uma função constante.

Analogamente, no caso em que $f_{2}$ é constante e $f_{1}$ não é monótona pelas hipóteses do Corolário 1.4 .1 devemos ter $a^{\prime} \leq 0$ onde $f_{2}^{\prime} \leq 0$ e $a^{\prime} \geq 0$ onde $f_{2}^{\prime} \geq 0$. Nesse caso, a condição $a^{\prime \prime} \leq 0$ é indica que $a$ deve ser constante.

Pela discussão acima concluimos que, se $\Omega$ é um domínio convexo delimitado pelas retas verticais $x=c, x=d$ e pelos gráficos da função convexa $y=f_{2}(x)$ e da função côncava $y=f_{1}(x)$, não monótonas, então uma função $a$ satisfazendo as hipóteses do Corolário 1.4.1 deve ser constante.

Valem observações análogas para o caso do Corolário 1.4.2.

Corolário 1.4.4 Seja $g:[c, d] \rightarrow[p, q]$ uma função de classe $\mathscr{C}^{2}$ tal que $g^{\prime}>0$ em $(c, d)$ e seja $h:[p, q] \rightarrow[c, d]$ sua inversa. Consideremos um domínio limitado convexo $\Omega$ dado por

$$
\Omega=\left\{(x, y) \in \mathbb{R}^{2} ; c<x<d, \quad p<y<g(x)\right\} .
$$

Sejam $a:[c, d] \rightarrow \mathbb{R}$ e $b:[p, q] \rightarrow \mathbb{R}$ de classe $\mathscr{C}^{2}$ tais que $a^{\prime \prime}(x) \leq 0$ em $[c, d]$ e $b^{\prime \prime}(y) \leq 0$ em $[p, q]$ e, além disso, $a^{\prime} g^{\prime} \leq 0$ e $b^{\prime} h^{\prime} \leq 0$ em seus respectivos domínios. Nessas condições, se $\phi$ é um equilíbrio não-constante do problema

$$
(P N)=\left\{\begin{array}{l}
u_{t}=\left(a^{2}(x) u_{x}\right)_{x}+\left(b^{2}(y) u_{y}\right)_{y}+f(u), \quad(x, y) \in \Omega, t>0 \\
\frac{\partial u}{\partial \nu}=a^{2}(x) u_{x} n_{1}+b^{2}(y) u_{y} n_{2}=0, \quad(x, y) \in \partial \Omega, t>0
\end{array}\right.
$$

então, $\phi$ é instável.

\section{Demonstração.}

Como o domínio é convexo já sabemos o sinal da primeira integral de (1.26) para a expressão $\Sigma=Q\left[a \phi_{x}\right]+Q\left[b \phi_{y}\right]$. Quanto à segunda integral de (1.26), pela fórmula 
(1.29), podemos escrever

$$
I=\int_{\Omega}\left(a \phi_{x}^{2} a^{2} a_{x x}+b \phi_{y}^{2} b^{2} b_{y y}\right)-\int_{\partial \Omega}\left(a \phi_{x}^{2} a^{2} a_{x} n_{1}+b \phi_{y}^{2} b^{2} b_{y} n_{2}\right) \mathrm{d} \sigma
$$

Com as hipóteses $a_{x x} \leq 0$ e $b_{y y} \leq 0$ a primeira integral da expressão acima é menor que ou igual a zero.

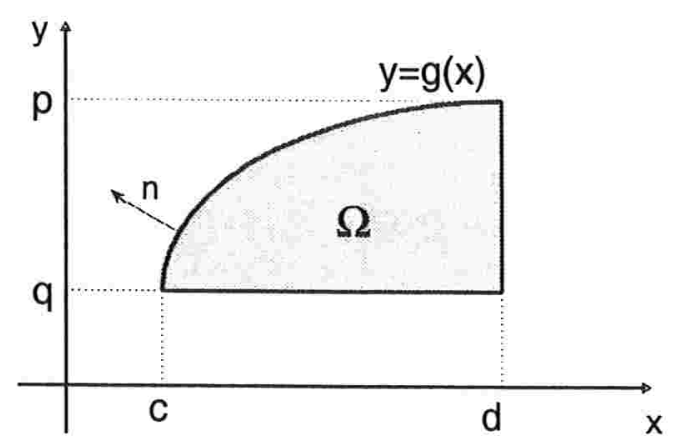

Figura 1.2: Exemplo de domínio convexo.

Considerando $\partial \Omega=\partial \Omega_{1} \cup \partial \Omega_{2} \cup \partial \Omega_{3}$ temos:

Em $\partial \Omega_{1}=\{(x, p) ; c \leq x \leq d\}$ o vetor normal exterior é $\vec{n}=(0,-1)$ e, nesse caso, temos $\int_{\partial \Omega_{1}} a \phi_{x}^{2} a^{2} a_{x} n_{1}=0$.

$\operatorname{Em} \partial \Omega_{2}=\{(d, y) ; p \leq y \leq g(d)\}$ o vetor normal exterior é $\vec{n}=(1,0)$. Pela condição de fronteira do equilíbrio temos $a^{2}(d) \phi_{x}(d, y)=0$, donde $\int_{\partial \Omega_{2}} a \phi_{x}^{2} a^{2} a_{x} n_{1}=0$.

Em $\partial \Omega_{3}=\{(c+d-x, g(c+d-x)) ; c<x<d\}$ o vetor normal exterior é $\vec{n}=$ $\frac{1}{\sqrt{1+g^{\prime 2}}}\left(-g^{\prime}(x), 1\right)$, donde

$$
\int_{\partial \Omega_{3}} a \phi_{x}^{2} a^{2} a_{x} n_{1}=\int_{c}^{d} a \phi_{x}^{2} a^{2}\left(-a_{x} g^{\prime}(x)\right) \mathrm{d} x
$$

a qual, por hipótese é menor que ou igual a zero. Com isso, temos $\int_{\Omega} a \phi_{x}^{2} a^{2} a_{x x}$ $\int_{\partial \Omega} a \phi_{x}^{2} a^{2} a_{x} n_{1} \leq 0$.

Analogamente, obtemos $\int_{\Omega} b \phi_{y}^{2} b^{2} b_{y y}-\int_{\partial \Omega} b \phi_{y}^{2} b^{2} b_{y} n_{2} \leq 0$. Dessas considerações obte$\operatorname{mos} \Sigma=Q\left[a \phi_{x}\right]+Q\left[b \phi_{y}\right] \leq 0$ donde segue a instabilidade do equilíbrio $\phi$.

Observação 1.4.2 No caso em que a matriz dos coeficientes de difusividades é dada por

$$
D(x, y)=\operatorname{diag}\left\{a^{2}(y), b^{2}(x)\right\}
$$


com a função $a$ dependendo somente de $y$ e a função $b$ dependendo somente de $x$, a matriz $\mathbb{A}$ é dada por

$$
\mathbb{A}=\left(\begin{array}{cc}
b^{2} a_{y}^{2} & -2 a a_{y} b b_{x} \\
-2 a a_{y} b b_{x} & a^{2} b_{x}^{2}
\end{array}\right)
$$

Nas hipóteses do Teorema 1.4.1 temos que a matriz $\mathbb{A}$ deve ser negativa semi-definida, donde, devem valer as seguintes desigualdades

$$
\left\{\begin{array}{r}
b^{2} a_{y}^{2} \leq 0 \\
a^{2} b_{x}^{2} \leq 0 \\
\operatorname{det} \mathbb{A} \geq 0 .
\end{array}\right.
$$

Nessas condições, para que as hipóteses do Teorema 1.4.1 estejam verificadas as funções difusividade $a$ e $b$ devem ser constantes.

\subsubsection{O caso tridimensional}

Consideremos o sistema representado por

$$
\left\{\begin{array}{l}
u_{t}=\operatorname{div}(D(x, y, z) \nabla u)+f(u), \quad(x, y, z) \in \Omega t>0 \\
\frac{\partial u}{\partial \nu}=\langle D \nabla u, \vec{n}\rangle=0, \quad x \in \partial \Omega, \quad t>0
\end{array}\right.
$$

onde $\Omega \subset \mathbb{R}^{3}$ é um domínio limitado cujo bordo $\partial \Omega$ é uma superfície lisa por partes,( de classe $\mathscr{C}^{2}$ por partes.) Neste caso, a matriz dos coeficientes de difusividade é dada por $D(x, y, z)=\operatorname{diag}\left\{a^{2}(x, y, z), b^{2}(x, y, z), c^{2}(x, y, z)\right\}$ onde $a, b, c: \Omega \longrightarrow \mathbb{R}$ são funções positivas de classe $\mathscr{C}^{2}$. e $f: \mathbb{R} \longrightarrow \mathbb{R}$ é uma função de classe $\mathscr{C}^{1}, \vec{n}$ indica o vetor normal exterior no ponto $(x, y, z) \in \partial \Omega$ e $\frac{\partial u}{\partial \nu}=\langle D \nabla u, \vec{n}\rangle$ a derivada co-normal de $u$ em $\partial \Omega$ indicada por

$$
\frac{\partial u}{\partial \nu}=\langle D \nabla u, \vec{n}\rangle=a^{2} \phi_{x} n_{1}+b^{2} \phi_{y} n_{2}+c^{2} \phi_{z} n_{3}
$$

$\operatorname{com} \vec{n}=\left(n_{1}, n_{2}, n_{3}\right)$.

Vamos analisar o sinal da expressão (1.27) que, para $N=3$, é representada por $\Sigma=Q\left[a \phi_{x}\right]+Q\left[b \phi_{y}\right]+Q\left[c \phi_{z}\right]$

Como interessa-nos abordar o caso das equações definidas em domínios convexos o sinal da primeira das integrais em (1.27) já é conhecido. Portanto, vamos estudar o sinal 
da segunda integral dessa expressão, a saber,

$$
\begin{aligned}
I=\int_{\Omega}[\langle D \nabla a, & \left.\nabla a\rangle \phi_{x}^{2}+\langle D \nabla b, \nabla b\rangle \phi_{y}^{2}+\langle D \nabla c, \nabla c\rangle \phi_{z}^{2}\right] \\
& -\int_{\Omega}\left\langle\left(D_{x} \nabla\left(a^{2} \phi_{x}\right)+D_{y} \nabla\left(b^{2} \phi_{y}\right)+D_{z} \nabla\left(c^{2} \phi_{z}\right)\right), \nabla \phi\right\rangle .
\end{aligned}
$$

Das três parcelas do integrando da primeira integral de $I$ em (1.31) temos

$$
\begin{aligned}
\langle D \nabla a, \nabla a\rangle \phi_{x}^{2} & =\left(a^{2}\left(a_{x}\right)^{2}+b^{2}\left(a_{y}\right)^{2}+c^{2}\left(a_{z}\right)^{2}\right) \phi_{x}^{2} \\
\langle D \nabla b, \nabla b\rangle \phi_{y}^{2} & =\left(a^{2}\left(b_{x}\right)^{2}+b^{2}\left(b_{y}\right)^{2}+c^{2}\left(b_{z}\right)^{2}\right) \phi_{y}^{2} \\
\langle D \nabla c, \nabla c\rangle \phi_{z}^{2} & =\left(a^{2}\left(c_{x}\right)^{2}+b^{2}\left(c_{y}\right)^{2}+c^{2}\left(c_{z}\right)^{2}\right) \phi_{z}^{2} .
\end{aligned}
$$

Por outro lado, para cada parcela da segunda integral de $I$ podemos escrever

$$
\left\langle D_{x} \nabla\left(a^{2} \phi_{x}\right), \nabla \phi\right\rangle=\left(a^{2}\right)_{x}\left(a^{2} \phi_{x}\right)_{x} \phi_{x}+\left(b^{2}\right)_{x}\left(a^{2} \phi_{x}\right)_{y} \phi_{y}+\left(c^{2}\right)_{x}\left(a^{2} \phi_{x}\right)_{z} \phi_{z}
$$

donde obtemos

$$
\left\{\begin{array}{l}
\left(a^{2}\right)_{x}\left(a^{2} \phi_{x}\right)_{x} \phi_{x}=a^{2} a_{x}^{2} \phi_{x}^{2}+\frac{\partial}{\partial x}\left(a\left(a \phi_{x}\right)^{2} a_{x}\right)-a \phi_{x}^{2} a^{2} a_{x x} \\
\left(b^{2}\right)_{x}\left(a^{2} \phi_{x}\right)_{y} \phi_{y}=4 b b_{x} a a_{y} \phi_{x} \phi_{y}+\frac{\partial}{\partial x}\left(b\left(a \phi_{y}\right)^{2} b_{x}\right)-b \phi_{y}^{2} a^{2} b_{x x}-2 a a_{x} b b_{x} \phi_{y}^{2}-a^{2} b_{x}^{2} \phi_{y}^{2} \\
\left(c^{2}\right)_{x}\left(a^{2} \phi_{x}\right)_{z} \phi_{z}=4 c c_{x} a a_{z} \phi_{x} \phi_{z}+\frac{\partial}{\partial x}\left(c\left(a \phi_{z}\right)^{2} c_{x}\right)-c \phi_{z}^{2} a^{2} c_{x x}-2 a a_{x} c c_{x} \phi_{z}^{2}-a^{2} c_{x}^{2} \phi_{z}^{2}
\end{array}\right.
$$

Analogamente, escrevemos

$$
\left\langle D_{y} \nabla\left(b^{2} \phi_{y}\right) \nabla \phi\right\rangle=\left(a^{2}\right)_{y}\left(b^{2} \phi_{y}\right)_{x} \phi_{x}+\left(b^{2}\right)\left(b^{2} \phi_{y}\right)_{y} \phi_{y}+\left(c^{2}\right)_{y}\left(b^{2} \phi_{y}\right)_{z} \phi_{z}
$$

donde, cada uma das parcelas se escreve como

$$
\left\{\begin{aligned}
\left(a^{2}\right)_{y}\left(b^{2} \phi_{y}\right)_{x} \phi_{x} & =4 a a_{y} b b_{x} \phi_{x} \phi_{y} \frac{\partial}{\partial y}\left(a\left(b \phi_{y}\right)^{2} a_{y}\right)-a \phi_{x}^{2} b^{2} a_{y y}-2 a a_{y} b b_{y} \phi_{x}^{2}-b^{2} a_{y}^{2} \phi_{x}^{2} \\
\left(b^{2}\right)\left(b^{2} \phi_{y}\right)_{y} \phi_{y} & =b^{2} b_{y}^{2} \phi_{y}^{2}+\frac{\partial}{\partial y}\left(b\left(b \phi_{y}\right)^{2} b_{y}\right)-b \phi_{y}^{2} b^{2} b_{y y} \\
\left(c^{2}\right)_{y}\left(b^{2} \phi_{y}\right)_{z} \phi_{z} & =4 c c_{y} b b_{z} \phi_{y} \phi_{z}+\frac{\partial}{\partial y}\left(c\left(b \phi_{z}\right)^{2} c_{y}\right)-c \phi_{z}^{2} b^{2} c_{y y}-2 b b_{y} c c_{y} \phi_{z}^{2}-b^{2} c_{y}^{2} \phi_{z}^{2}
\end{aligned}\right.
$$

e

$$
\left\langle D_{z} \nabla\left(c^{2} \phi_{z}\right), \nabla \phi\right\rangle=\left(a^{2}\right)_{z}\left(c^{2} \phi_{z}\right)_{x} \phi_{x}+\left(b^{2}\right)\left(c^{2} \phi_{z}\right)_{y} \phi_{y}+\left(c^{2}\right)_{z}\left(c^{2} \phi_{z}\right)_{z} \phi_{z}
$$

onde cada parcela se expressa como

$$
\left\{\begin{array}{l}
\left(a^{2}\right)_{z}\left(c^{2} \phi_{z}\right)_{x} \phi_{x}=4 a a_{z} c c_{x} \phi_{x} \phi_{z}+\frac{\partial}{\partial z}\left(a\left(c \phi_{x}\right)^{2} a_{z}\right)-a \phi_{x}^{2} c^{2} a_{z z}-2 a a_{z} c c_{z} \phi_{x}^{2}-c^{2} a_{z}^{2} \phi_{x}^{2} \\
\left(b^{2}\right)_{z}\left(c^{2} \phi_{z}\right)_{y} \phi_{y}=4 b b_{z} c c_{y} \phi_{y} \phi_{z}+\frac{\partial}{\partial z}\left(b\left(c \phi_{y}\right)^{2} b_{z}\right)-b \phi_{y}^{2} c^{2} b_{z z}-2 b b_{z} c c_{z} \phi_{y}^{2}-c^{2} b_{z}^{2} \phi_{y}^{2} \\
\left(c^{2}\right)_{z}\left(c^{2} \phi_{z}\right)_{z} \phi_{z}=c^{2} c_{z}^{2} \phi_{z}^{2}+\frac{\partial}{\partial z}\left(c\left(c \phi_{z}\right)^{2} c_{z}\right)-c \phi_{z}^{2} c^{2} c_{z z}
\end{array}\right.
$$


Finalmente, com as expressões obtidas nas manipulações acima podemos escrever as integrais da fórmula (1.31) alternativamente como

$$
\begin{aligned}
I=-\int_{\partial \Omega}\left[a \phi_{x}^{2}\right. & \left.\frac{\partial a}{\partial \nu}+b \phi_{y}^{2} \frac{\partial b}{\partial \nu}+c \phi_{z}^{2} \frac{\partial c}{\partial \nu}\right] \mathrm{d} \sigma \\
& +\int_{\Omega}\left[a \phi_{x}^{2} D \Delta a+b \phi_{y}^{2} D \Delta b+c \phi_{z}^{2} D \Delta c\right]+2 \int_{\Omega}\langle\mathbb{A} \nabla \phi, \nabla \phi\rangle
\end{aligned}
$$

onde estamos considerando as seguintes expressões $D \Delta a=a^{2} a_{x x}+b^{2} a_{y y}+c^{2} a_{z z}, D \Delta b=$ $a^{2} b_{x x}+b^{2} b_{y y}+c^{2} b_{z z}$, e $D \Delta c=a^{2} c_{x x}+b^{2} c_{y y}+c^{2} c_{z z}$. A forma quadrática $\langle\mathbb{A} \xi, \xi\rangle$ é definida para todo $(x, y, z) \in \Omega$ e todo $\xi \in \mathbb{R}^{3}$, com a matriz $\mathbb{A}$ é dada por

$$
\left(\begin{array}{ccc}
a a_{y} b b_{y}+a a_{z} c c_{z}+b^{2} a_{y}^{2}+c^{2} a_{z}^{2} & -2 a a_{y} b b_{x} & -2 a a_{z} c c_{x} \\
-2 a a_{y} b b_{x} & a a_{x} b b_{x}+b b_{z} c c_{z}+a^{2} b_{x}^{2}+c^{2} b_{z}^{2} & -2 b b_{z} c c_{y} \\
-2 a a_{z} c c_{x} & -2 b b_{z} c c_{y} & a a_{x} c c_{x}+b b_{y} c c_{y}+a^{2} c_{x}^{2} b^{2} c_{y}^{2}
\end{array}\right)
$$

De maneira análoga ao caso bidimensional podemos enunciar e provar o seguinte teorema

Teorema 1.4.2 Seja $\phi$ um equilíbrio do problema (1.30) onde $\Omega \subset \mathbb{R}^{3}$ é um domínio limitado convexo com bordo $\partial \Omega$ uma superfície de classe $\mathscr{C}^{2}$ por partes e $D: \Omega \rightarrow \mathbb{M}_{3}(\mathbb{R})$ é uma matriz diagonal satisfazendo as seguintes condições: $D \Delta a \leq 0, D \Delta b \leq 0$ e $D \Delta c \leq 0$ em $\Omega, \frac{\partial a}{\partial \nu} \geq 0, \frac{\partial b}{\partial \nu} \geq 0$ e $\frac{\partial b}{\partial \nu} \geq 0$ em $\partial \Omega$ e a forma quadrática definida pela matriz $\mathbb{A}$, dada pela expressão (1.33), satisfaz $\langle\mathbb{A} \xi, \xi\rangle \leq 0$ em $\Omega, \forall \xi \in \mathbb{R}^{3}$. Nessas condições, se $\phi$ é um equilibrio não-constante do problema (1.30), então $\phi$ é instável.

Demonstração. A demonstração é análoga à do caso bidimensional e será omitida.

Alguns exemplos de problemas com difusividades variáveis definidos em domínios convexos do espaço $\mathbb{R}^{3}$ são dados conforme o corolário seguinte.

\section{Corolário 1.4.5 Seja}

$$
\Omega=\left\{(x, y, z) \in \mathbb{R}^{3} ; c<x<d, m<y<n, f_{1}(x)<z<f_{2}(x)\right\}
$$

onde $f_{1}, f_{2}:[c, d] \longrightarrow \mathbb{R}$ são funções de classe $\mathscr{C}^{2}$. Seja $a:[c, d] \rightarrow \mathbb{R}$ de classe $\mathscr{C}^{2}$ tal que $a^{\prime \prime} \leq 0$, e $a^{\prime} f_{2}^{\prime} \leq 0$ e $a^{\prime} f_{1}^{\prime} \geq 0$ em $(c, d)$. Nessas condições, se $\phi$ é um equilibrio 
não-constante do problema

$$
\left\{\begin{array}{l}
u_{t}=\left(a^{2} u_{x}\right)_{x}+u_{y y}+u_{z z}+f(u),(x, y, z) \in \Omega, t>0 \\
\frac{\partial u}{\partial \nu}=a^{2} u_{x} n_{1}+u_{y} n_{2}+u_{z} n_{3}=0,(x, y, z) \in \partial \Omega, t>0
\end{array}\right.
$$

então, $\phi$ é instável.

Demonstração. Seja $\phi$ equilíbrio não-constante do problema dado. Como o domínio é convexo com fronteira lisa por partes, do Teorema 1.2.1, segue que a primeira integral da expressão (1.27), onde, $\Sigma=Q\left[a \phi_{x}\right]+Q\left[\phi_{y}\right]+Q\left[\phi_{z}\right]$, é menor que ou igual a zero. Pelas condições do problema, a matriz $\mathbb{A}$ definida em (1.33) é nula, donde a forma quadrática associada também é nula e, nesse caso, a segunda integral da expressão $\Sigma$, segundo a fórmula (1.32), é dada por

$$
I=\int_{\Omega} a \phi_{x}^{2} a^{2} a_{x x} \mathrm{~d} x-\int_{\partial \Omega} a \phi_{x}^{2} a^{2} a_{x} n_{1} \mathrm{~d} \sigma
$$

Como $a^{\prime \prime} \leq 0$ a primeira das integrais de $I$ é menor que ou igual a zero. Quanto ao sinal da segunda integral observamos que a fronteira $\partial \Omega$ se decompôe como

$$
\partial \Omega=\partial \Omega_{1} \cup \partial \Omega_{2} \cup \partial \Omega_{3} \cup \partial \Omega_{4} \cup \partial \Omega_{5} \cup \partial \Omega_{6}
$$

Em $\partial \Omega_{1}=\left\{(d, y, z) ; m<y<n, f_{1}(x)<z<f_{2}(x)\right\}$ o vetor normal exterior é $\vec{n}=$ $(1,0,0)$ e, pela condição de fronteira, $a^{2}(d) \phi_{x}(d, y, z)=0$, donde, $\int_{\partial \Omega_{1}} a \phi_{x}^{2} a^{2} a_{x} n_{1}=0$. Analogamente, em $\partial \Omega_{2}=\left\{(c, y, z) ; m<y<n, f_{1}(x)<z<f_{2}(x)\right\}$ o vetor normal exterior é $\vec{n}=(-1,0,0)$ e pela condição de fronteira, temos $\int_{\partial \Omega_{2}} a \phi_{x}^{2} a^{2} a_{x} n_{1}=0$.

Em $\partial \Omega_{3}=\left\{(x, m, z), c<x<d, f_{1}(x)<z<f_{2}(x)\right\}$ e em $\partial \Omega_{4}=\{(x, n, z), c<x<$ $\left.d, f_{1}(x)<z<f_{2}(x)\right\}$ temos $\vec{n}=(0,1,0)$ e $\vec{n}=(0,-1,0)$ respectivamente, os vetores normais exteriores. Em cada um dos casos $n_{1}=0$ donde temos

$$
\int_{\partial \Omega_{3}} a \phi_{x}^{2} a^{2} a_{x} n_{1}=\int_{\partial \Omega_{4}} a \phi_{x}^{2} a^{2} a_{x} n_{1}=0 .
$$

Em $\partial \Omega_{5}=\left\{(x, y, z) ; c<x<d, m<y<n, z=f_{2}(x)\right\}$ o vetor normal exterior é $\vec{n}=\frac{1}{\sqrt{1+f_{2}^{\prime 2}}}\left(-f_{2}^{\prime}, 0,1\right)$ e, relativamente à parametrização $\sigma=(x, y, f(x)), \quad c<x<$ $d, m<y<n$ temos

$$
\mathrm{d} \sigma=\left\|\frac{\partial \sigma}{\partial x} \times \frac{\partial \sigma}{\partial y}\right\| \mathrm{d} x \mathrm{~d} y=\sqrt{1+f_{2}^{\prime 2}} \mathrm{~d} x \mathrm{~d} y .
$$


Como, por hipótese, $a^{\prime} f_{2}^{\prime} \leq 0$ vem

$$
\int_{\partial \Omega_{5}} a \phi_{x}^{2} a^{2} a_{x} n_{1} \mathrm{~d} \sigma=\int_{m}^{n} \int_{c}^{d} a \phi_{x}^{2} a^{2}\left[-a^{\prime}(x) f_{2}^{\prime}(x)\right] \mathrm{d} x \mathrm{~d} y \geq 0 .
$$

Analogamente, em $\partial \Omega_{6}=\left\{(x, y, z) ; c<x<d, m<y<n, z=f_{1}(x)\right\}$ com a hipótese $a^{\prime} f_{1}^{\prime} \geq 0$ concluimos que

$$
\int_{\partial \Omega_{6}} a \phi_{x}^{2} a^{2} a_{x} n_{1} \geq 0
$$

e, com isso, temos $\Sigma=Q\left[a \phi_{x}\right]+Q\left[\phi_{y}\right]+Q\left[\phi_{z}\right] \leq 0$, donde segue a instabilidade do equilíbrio $\phi$.

Para finalizar esta seção comentamos que, no caso geral, para a determinação da instabilidade de equilíbrios de problemas definidos em domínios limitados convexos do $\mathbb{R}^{N}$, a análise do sinal da expressão $\Sigma$ dada em (1.27) depende somente do sinal da integral $I$ sobre $\Omega$. Generalizando o que foi feito para $N=2$ e $N=3$, essa integral pode ser expressa, por

$$
I=-\int_{\partial \Omega} \sum_{j} a_{j} \phi_{x_{j}}^{2} \frac{\partial a_{j}}{\partial \nu}+\int_{\Omega} \sum_{j} a_{j} D \Delta a_{j}+2 \int_{\Omega}\langle\mathbb{A} \nabla \phi, \nabla \phi\rangle
$$

onde as expressões $A \Delta a_{j}, 1 \leq j \leq N$, e a forma quadrática $\langle\mathbb{A} \nabla \phi, \nabla \phi\rangle$ são definidas de maneira análoga à dos casos considerados. Podemos assim, generalizar os resultados obtidos para os casos particulares tratados acima.

\subsection{Coeficientes do Tipo $k(x) I_{N}$}

$\mathrm{Na}$ perspectiva de estudar a influência dos coeficientes de difusão sobre a estabilidade dos equilíbrios da equação (1.1), vamos considerar o caso em que a matriz dos coeficientes é da forma $A(x)=k(x) I_{N}$ onde $I_{N}$ indica a matriz identidade de ordem $N$.

Seja o problema para as equações com coeficientes variáveis do tipo

$$
\left\{\begin{array}{l}
u_{t}=\operatorname{div}(k(x) \nabla u)+f(u), \quad x \in \Omega \quad t>0, \\
\frac{\partial u}{\partial \vec{n}}=\langle\nabla u, \vec{n}\rangle=0, \quad x \in \partial \Omega, \quad t \geq 0,
\end{array}\right.
$$

onde $\Omega \subset \mathbb{R}^{N}$ um subconjunto aberto, conexo, limitado com a fronteira $\partial \Omega$ de classe $\mathscr{C}^{2}, f: \mathbb{R} \longrightarrow \mathbb{R}$ é uma função diferenciável satisfazendo condições para que o problema 
(1.34) seja bem posto e que a solução pertença à classe de regularidade adequada, $k: \Omega \longrightarrow \mathbb{R}$ é uma aplicação de classe $\mathscr{C}^{2}, k(x) \geq k_{0}>0$, para todo $x \in \Omega, \vec{n}$ indica o vetor normal exterior no ponto $x \in \partial \Omega$ e $\frac{\partial u}{\partial \vec{n}}=\langle\nabla u, \vec{n}\rangle$ indica a derivada normal de $u$ em $\partial \Omega$.

Seja $\phi: \bar{\Omega} \longrightarrow \mathbb{R}$ um equilíbrio não-constante de (1.34) e consideremos o operador linearizado em torno de $\phi$ dado por $\mathscr{L}[w]=-\operatorname{div}(k(x) \nabla w)-f^{\prime}(\phi) w, w \in \mathbf{H}^{2}(\Omega)$, $\frac{\partial w}{\partial \vec{n}}=0$ em $\partial \Omega$. A fim de determinar a instabilidade do equilíbrio $\phi$, vamos estimar o sinal do autovalor principal do operador $\mathscr{L}$. Para tanto, consideremos o funcional quadrático $Q: \mathbf{H}^{1}(\Omega) \rightarrow \mathbb{R}$ dado por

$$
Q[u]=\int_{\Omega}\left(k(x)\|\nabla u\|^{2}-f^{\prime}(\phi) u^{2}\right), \quad \forall u \in \mathbf{H}^{1}(\Omega) .
$$

Vamos escolher alguma funções com propriedades conhecidas a partir das quais possamos estabelecer condições para a avaliação do sinal do autovalor principal de $\mathscr{L}$ por meio de uma expressão envolvendo o funcional $Q$. Como $\operatorname{div}(u k(x) \nabla u)=$ $u \operatorname{div}(k(x) \nabla u)+k(x)\|\nabla u\|^{2}$ podemos re-escrever

$$
Q[u]=\int_{\Omega}\left(\operatorname{div}(k(x) u \nabla u)-u \operatorname{div}(k(x) \nabla u)-f^{\prime}(\phi) u^{2}\right) \mathrm{d} x,
$$

donde

$$
Q[u]=\frac{1}{2} \int_{\partial \Omega} k(x) \frac{\partial u^{2}}{\partial \vec{n}} \mathrm{~d} \sigma-\int_{\Omega}\left(u \operatorname{div}(k(x) \nabla u)+f^{\prime}(\phi) u^{2}\right) \mathrm{d} x .
$$

Para cada $j, 1 \leq j \leq N$, consideremos o valor do funcional $Q$ na função $u=\phi_{x_{j}}$

$$
Q\left[\phi_{x_{j}}\right]=\frac{1}{2} \int_{\partial \Omega} k(x) \frac{\partial\left(\phi_{x_{j}}\right)^{2}}{\partial \vec{n}} \mathrm{~d} \sigma-\int_{\Omega}\left(\phi_{x_{j}} \operatorname{div}\left(k(x) \nabla \phi_{x_{j}}\right)+f^{\prime}(\phi) \phi_{x_{j}}^{2}\right) \mathrm{d} x .
$$

Da condição de equilíbrio $\operatorname{div}(k(x) \nabla \phi)+f(\phi)=0$, derivando em relação a $x_{j}$, vem $\operatorname{div}\left(k_{x_{j}} \nabla \phi\right)+\operatorname{div}\left(k \nabla \phi_{x_{j}}\right)+f^{\prime}(\phi) \phi_{x_{j}}=0$, donde segue

$$
\begin{aligned}
-\phi_{x_{j}} \operatorname{div}\left(k \nabla \phi_{x_{j}}\right)-f^{\prime}(\phi) \phi_{x_{j}}^{2} & =\phi_{x_{j}} \operatorname{div}\left(k_{x_{j}} \nabla \phi\right) \\
& =\operatorname{div}\left(\phi_{x_{j}} k_{x_{j}} \nabla \phi\right)-\left\langle k_{x_{j}} \nabla \phi, \nabla \phi_{x_{j}}\right\rangle \\
& =\operatorname{div}\left(\phi_{x_{j}} k_{x_{j}} \nabla \phi\right)-\frac{1}{2} k_{x_{j}} \frac{\partial}{\partial x_{j}}\left(\|\nabla \phi\|^{2}\right) .
\end{aligned}
$$

Desse modo, para cada $j$, podemos expressar

$$
-\phi_{x_{j}} \operatorname{div}\left(k \nabla \phi_{x_{j}}\right)-f^{\prime}(\phi) \phi_{x_{j}}^{2}=\operatorname{div}\left(\phi_{x_{j}} k_{x_{j}} \nabla \phi\right)-\frac{1}{2} \frac{\partial}{\partial x_{j}}\left(k_{x_{j}}\|\nabla \phi\|^{2}\right)+\frac{1}{2}\|\nabla \phi\|^{2} k_{x_{j} x_{j}} .
$$


Portanto, para cada $j, 1 \leq j \leq N$, a segunda integral na expressão (1.35) é dada por

$$
\int_{\Omega}\left[\operatorname{div}\left(\phi_{x_{j}} k_{x_{j}} \nabla \phi\right)-\frac{1}{2} \frac{\partial}{\partial x_{j}}\left(k_{x_{j}}\|\nabla \phi\|^{2}\right)+\frac{1}{2}\|\nabla \phi\|^{2} k_{x_{j} x_{j}}\right] .
$$

Usando o Teorema da Divergência escrevemos essa integral como

$$
\int_{\partial \Omega} \phi_{x_{j}} k_{x_{j}} \frac{\partial \phi}{\partial \vec{n}}+\int_{\partial \Omega}\left[-\frac{1}{2} \frac{\partial}{\partial x_{j}}\left(k_{x_{j}}\|\nabla \phi\|^{2}\right)+\frac{1}{2}\|\nabla \phi\|^{2} k_{x_{j} x_{j}}\right] .
$$

Finalmente, da condição de equilíbrio do problema (1.34) temos $\frac{\partial \phi}{\partial \vec{n}}=0$ em $\partial \Omega$ donde podemos escrever

$$
-\frac{1}{2} \int_{\Omega} \frac{\partial}{\partial x_{j}}\left(k_{x_{j}}\|\nabla \phi\|^{2}\right)+\frac{1}{2} \int_{\Omega}\|\nabla \phi\|^{2} k_{x_{j} x_{j}} .
$$

Com os resultados obtidos acima, para cada $j$, podemos reescrever a expressão (1.35) como

$Q\left[\phi_{x_{j}}\right]=\frac{1}{2} \int_{\partial \Omega} k(x) \frac{\partial\left(\phi_{x_{j}}\right)^{2}}{\partial \vec{n}} \mathrm{~d} \sigma-\frac{1}{2} \int_{\Omega} \frac{\partial}{\partial x_{j}}\left(k_{x_{j}}\|\nabla \phi\|^{2}\right)+\frac{1}{2} \int_{\Omega}\|\nabla \phi\|^{2} k_{x_{j} x_{j}}, \quad 1 \leq j \leq N$.

Somando em $j$

$$
\sum_{j=1}^{N} Q\left[\phi_{x_{j}}\right]=\frac{1}{2} \int_{\partial \Omega} k(x) \frac{\partial(\|\nabla \phi\|)^{2}}{\partial \vec{n}} \mathrm{~d} \sigma-\frac{1}{2} \int_{\Omega} \operatorname{div}\left(\|\nabla \phi\|^{2} \nabla k\right)+\frac{1}{2} \int_{\Omega}\|\nabla \phi\|^{2} \Delta k .
$$

Mais uma aplicação do Teorema da Divergência na segunda integral da expressão acima nos dá

$$
\sum_{j=1}^{N} Q\left[\phi_{x_{j}}\right]=\frac{1}{2} \int_{\partial \Omega} k(x) \frac{\partial}{\partial \vec{n}}\left(\|\nabla \phi\|^{2}\right) \mathrm{d} \sigma-\frac{1}{2} \int_{\partial \Omega}\|\nabla \phi\|^{2} \frac{\partial k}{\partial \vec{n}}+\frac{1}{2} \int_{\Omega}\|\nabla \phi\|^{2} \Delta k
$$

A expressão (1.36) será bastante útil no estudo da instabilidade dos equilíbrios do problema (1.34), uma vez que a análise dos sinais dos autovalores do operador linearizado $\mathscr{L}$ pode ser reduzido ao estudo dos sinais das integrais dessa expressão. Por (1.36) percebemos a influência da propriedade geométrica do domínio uma vez que, quando o domínio $\Omega$ é convexo a primeira integral da expressão é menor que ou igual a zero. Além disso, também estão presentes na expressão, as condições sobre a função difusividade $k$. 


\subsection{O Problema Escalar Heterogêneo}

Seja o problema definido num intervalo $\Omega$ fixado da reta que, pelo fato de ser convexo, essa particularidade geométrica do domínio não será uma propriedade que vá interferir no resultado a ser obtido. Em Smoller [39] e em Smoller e Wassermann [40], por exemplo, são consideradas equações definidas no intervalo $(-l, l)$ com difusividades constantes e são estudados problemas de bifurcação usando o comprimento $l$ do intervalo $\Omega$ como parâmetro. Nosso enfoque aqui será sobre a influência da concavidade da função difusividade sobre a estabilidade dos equilíbrios não-constantes da equação dada.

Consideremos o seguinte problema de valor de fronteira

$$
\left\{\begin{aligned}
u_{t} & =\left(a(x) u_{x}\right)_{x}+f(u), \quad x \in \Omega, t>0 \\
u_{x}(0, t) & =u_{x}(l, t)=0, \quad t>0,
\end{aligned}\right.
$$

onde $\Omega=(0, l) \subset \mathbb{R}, a: \Omega \longrightarrow \mathbb{R}$ é uma função de classe $\mathscr{C}^{2}$ satisfazendo $a(x) \geq a_{0}>0$ para todo $x \in \Omega$ e $f: \mathbb{R} \longrightarrow \mathbb{R}$ é uma função de classe $\mathscr{C}^{1}$.

Seja $\phi: \Omega \longrightarrow \mathbb{R}$ um equilíbrio não constante de (1.37), isto é, $\phi=\phi(x)$ é solução do seguinte problema

$$
\left\{\begin{aligned}
\left(a(x) \phi_{x}\right)_{x}+f(\phi) & =0, \quad 0<x<l \\
\phi^{\prime}(0)=\phi^{\prime}(l) & =0 .
\end{aligned}\right.
$$

O operador linear correspondente ao equilíbrio $\phi$ é dado por $\mathscr{L}[u]=-\left(a u^{\prime}\right)^{\prime}-f^{\prime}(\phi) u$ para $u \in \mathbf{H}^{2}(\Omega)$ satisfazendo $u^{\prime}(0)=0$ e $u^{\prime}(l)=0$. Para o estudo do sinal dos autovalores do operador linear temos a forma quadrática definida por

$$
\langle\mathscr{L}[u], u\rangle=\int_{0}^{l}-u\left(\left(a u^{\prime}\right)^{\prime}+f^{\prime}(\phi) u\right) .
$$

Usando uma integração por partes obtemos a expressão

$$
\langle\mathscr{L}[u], u\rangle=-\left.u\left(a u^{\prime}\right)\right|_{0} ^{l}+\int_{0}^{l}\left(a\left(u^{\prime}\right)^{2}-f^{\prime}(\phi) u^{2}\right) .
$$

Definindo o funcional $Q$ por $Q[u]=\int_{0}^{l}\left(a\left(u^{\prime}\right)^{2}-f^{\prime}(\phi) u^{2}\right), u \in \mathbf{H}^{1}(0, l)$, temos, em conseqüência,

$$
Q[u]=\langle\mathscr{L}[u], u\rangle, \forall u \in \mathbf{H}^{2}(0, l), u^{\prime}(0)=u^{\prime}(l)=0
$$


Em particular, considerando um equilíbrio não constante $\phi=\phi(x)$ do sistema (1.37) temos

$$
Q\left[\phi^{\prime}\right]=\int_{0}^{l}-\phi^{\prime}\left(\left(a \phi^{\prime \prime}\right)^{\prime}+f^{\prime}(\phi) \phi^{\prime}\right) \mathrm{d} x
$$

Da condição de equilíbrio $\left(a \phi^{\prime}\right)^{\prime}+f(\phi)=0$ vem

$$
\left(a \phi^{\prime \prime}\right)+a^{\prime} \phi^{\prime}+f(\phi)=0 .
$$

Derivando mais uma vez a expressão obtemos

$$
\left(a \phi^{\prime \prime}\right)^{\prime}+\left(a^{\prime} \phi^{\prime}\right)^{\prime}+f^{\prime}(\phi) \phi^{\prime}=0
$$

multiplicando por $\phi^{\prime}$ podemos escrever

$$
-\phi^{\prime}\left[\left(a \phi^{\prime \prime}\right)^{\prime}+f^{\prime}(\phi) \phi^{\prime}\right]=\phi^{\prime}\left(a^{\prime} \phi^{\prime}\right)^{\prime}=a^{\prime \prime}\left(\phi^{\prime}\right)^{2}+a^{\prime} \phi^{\prime} \phi^{\prime \prime}
$$

Portanto,

$$
\begin{aligned}
Q\left[\phi^{\prime}\right] & =\int_{0}^{l}\left(a^{\prime \prime}\left(\phi^{\prime}\right)^{2}+a^{\prime} \phi^{\prime} \phi^{\prime \prime}\right) \\
& =\int_{0}^{l} a^{\prime \prime}\left(\phi^{\prime}\right)^{2}+\frac{1}{2} \int_{0}^{l} a^{\prime}\left(\left(\phi^{\prime}\right)^{2}\right)^{\prime} \\
& =\frac{1}{2} \int_{0}^{l} a^{\prime \prime}\left(\phi^{\prime}\right)^{2}+\frac{1}{2} \int_{0}^{l}\left(a^{\prime}\left(\phi^{\prime}\right)^{2}\right)^{\prime} .
\end{aligned}
$$

Substituindo em (1.38), temos

$$
Q\left[\phi^{\prime}\right]=\left.\frac{1}{2} a\left(\phi^{\prime 2}\right)^{\prime}\right|_{0} ^{l}+\left.\frac{1}{2}\left(\phi^{\prime}\right)^{2} a^{\prime}\right|_{0} ^{l}+\frac{1}{2} \int_{0}^{l} a^{\prime \prime}\left(\phi^{\prime}\right)^{2} .
$$

Notamos que, para o caso $N=1$, a expressão (1.36) é afetada pelas características geométricas unidimensionais do domínio e pelas propriedades das funções definidas em intervalos da reta. É imediato verificar que

$$
I_{1}=\left.\frac{1}{2} a(x)\left(\left(\phi^{\prime}(x)\right)^{2}\right)^{\prime}\right|_{0} ^{l}=0
$$

$\mathrm{e}$

$$
I_{2}=\left.\frac{1}{2} a^{\prime}(x)\left(\phi^{\prime}(x)\right)^{2}\right|_{0} ^{l}=0
$$

Quando a difusividade $a$ satisfaz $a^{\prime \prime}(x) \leq 0$ em $(0, l)$ então temos

$$
I_{3}=\frac{1}{2} \int_{0}^{l} a^{\prime \prime}(x)\left(\phi^{\prime}(x)\right)^{2} \mathrm{~d} x \leq 0 .
$$

Nessas condições, temos $Q\left[\phi^{\prime}\right]=I_{1}+I_{2}+I_{3} \leq 0$.

Tendo em vista a discussão feita acima e pelo Teorema 1.1.1 temos o seguinte 
Teorema 1.6.1 Se $\phi=\phi(x)$ é um equilibrio não-constante de (1.37) e se $a^{\prime \prime}(x) \leq 0$ para todo $x$ em $\Omega$, então $\phi$ é instável.

Observação 1.6.1 O resultado acima aparece em Chipot e Hale [9] com a hipótese de que $a^{\prime \prime}(x) \leq 0$ em $\Omega$ e em Yanagida [42] que obtém um resultado um pouco mais geral uma vez que exige $a^{\prime \prime}(x) \leq \frac{\left(a^{\prime}\right)^{2}}{2 a}$. Esses resultados representam uma generalização, no caso $N=1$, do resultado de Chafee [8]. Notamos ainda que Yanagida mostra que, para a equação $u_{t}=\left(a^{2}(x) u_{x}\right)_{x}+f(u), 0<x<1$, a existência de um ponto $x_{0} \in(0,1)$ tal que $a^{\prime \prime}\left(x_{0}\right)>0$ permite definir uma função $f: \mathbb{R} \longrightarrow \mathbb{R}$ de modo que a equação dada admita um equilíbrio não-constante, $\phi:(0,1) \longrightarrow \mathbb{R}$, estável.

\subsection{Domínios Convexos}

Consideremos o problema da estabilidade dos equilíbrios da equação (1.34) nos casos em que o domínio de definição $\Omega \subset \mathbb{R}^{N}$ é um subconjunto convexo limitado com bordo $\partial \Omega$ suave. Queremos determinar condições sobre a função difusividade $k$ de modo possamos garantir, para domínios convexos dados, a instabilidade de equilíbrios não-constantes do problema (1.34). Talvez a colocação do problema num aspecto mais geral seria propor uma questão do tipo:

Dados um domínio $\Omega$ convexo e $f: \mathbb{R} \rightarrow \mathbb{R}$ não-convexa para quais funções difusividade $k$ podemos afirmar que todo equilíbrio não-constante $\phi$ do problema (1.34) é instável?

Parte da resposta a essa questão pode ser obtida analisando os sinais das integrais da expressão (1.36). As três integrais que compõem a expressão (1.36) revelam uma estreita influência das características geométricas do domínio $\Omega$ e do equilíbrio $\phi$ sobre a estabilidade do equilíbrio. De certa forma essa influência já foi explorada quando abordamos o caso das equações com coeficientes constantes. No caso das equações com coeficientes variáveis um resultado no sentido de explorar esse tipo de influência vem do fato de que se $\Omega$ é um domínio convexo, então, pelo Corolário 1.2.2, a primeira integral da expressão (1.36) é menor que ou igual a zero.

Uma vez determinado um tipo de domínio $\Omega$ o sinal da primeira integral é conhecido. Várias considerações sobre os integrandos das duas outras integrais que compõem a 
expressão (1.36) podem ser feitas no sentido de determinar o seu sinal. Como estamos interessados em estabelecer condições sobre a difusividade $k$, uma primeira tentativa seria exigir que os respectivos integrandos fossem negativos ou nulos. Nessas condições, deveríamos ter $\Delta k \leq 0$ em $\Omega$ e $\frac{\partial k}{\partial \vec{n}} \geq 0$ em $\partial \Omega$. Da primeira condição sobre $k$ em $\Omega$ viria

$$
\int_{\Omega} \Delta k \leq 0
$$

Mas, essa situação implicaria

$$
\int_{\partial \Omega} \frac{\partial k}{\partial \vec{n}} \leq 0
$$

o que, para se compatibilizar com a condição imposta para $k$ em $\partial \Omega$ levaria a admitir $\frac{\partial k}{\partial \vec{n}}=0 \mathrm{em} \partial \Omega$. Assim, numa primeira abordagem, as exigências acima indicam que, para que as integrais satisfaçam as desigualdades estabelecidas seria suficiente impor que a função difusividade $k$ satisfizesse as seguintes condições

$$
\left\{\begin{aligned}
\Delta k & \leq 0, \text { em } \Omega \\
\frac{\partial k}{\partial \vec{n}} & =0, \text { em } \partial \Omega .
\end{aligned}\right.
$$

Nesse caso, as funções $k$ que verificam as condições acima são super-harmônicas em $\Omega$ com derivadas normais nulas em $\partial \Omega$. Ora, nessa situação, a condição de fronteira implica $\int_{\partial \Omega} \frac{\partial k}{\partial \vec{v}}=\int_{\Omega} \Delta k=0$. Como $\Delta k$ é contínua e satisfaz $\Delta k \leq 0$ em $\Omega$ devemos ter $\Delta k=0$, donde segue que $k$ deve ser constante. Esta é precisamente a situação considerada por Matano [33] e Casten e Holland [7].

Observamos que, em comparação com o caso de coeficientes variáveis dados pela matriz diagonal $D$, o caso presente é mais restritivo. Num sentido mais geral, exigindo apenas a convexidade do domínio $\Omega$ e a não convexidade do termo não-linear $f$, a caracterização de uma classe de funções difusividade $k$, que satisfaça as exigências acima e que responda à pergunta inicial é a clase das constantes positivas. Procuremos estabelecer algumas condições complementares para tornar o problema mais tratável com as ferramentas de que dispomos. Nesse sentido, embora de pouca utilidade prática, mas para efeito de registro enunciamos o resultado seguinte.

Proposição 1.7.1 Se $\phi \in \mathscr{C}^{2}(\Omega) \cap \mathscr{C}(\bar{\Omega})$ é um equilíbrio não-constante de (1.34) e se $k: \Omega \rightarrow \mathbb{R}$ é uma função de classe $\mathscr{C}^{1}$ tal que $\int_{\Omega} k \Delta\left(\|\nabla \phi\|^{2}\right) \leq 0$, então $\phi$ é instável. 
Demonstração. Como $\Omega$ é convexo e $\phi$ é um equilíbrio de (1.34), do Corolário 1.2.2 temos que

$$
\int_{\partial \Omega} \frac{\partial}{\partial \vec{n}}\left(\|\nabla \phi\|^{2}\right) \leq 0
$$

e, pelo Teorema da Divergência, temos

$$
\int_{\Omega} \Delta\left(\|\nabla \phi\|^{2}\right) \leq 0
$$

Da expressão (1.36) temos

$$
\sum_{j=1}^{N} Q\left[\phi_{x_{j}}\right]=\frac{1}{2} \int_{\partial \Omega}\left[k(x) \frac{\partial}{\partial \vec{n}}\left(\|\nabla \phi\|^{2}\right)-\|\nabla \phi\|^{2} \frac{\partial k}{\partial \vec{n}}\right] \mathrm{d} \sigma+\frac{1}{2} \int_{\Omega}\|\nabla \phi\|^{2} \Delta k .
$$

Usando a segunda identidade de Green podemos escrever

$$
\sum_{j=1}^{N} Q\left[\phi_{x_{j}}\right]=\frac{1}{2} \int_{\Omega}\left[k(x) \Delta\left(\|\nabla \phi\|^{2}\right)-\|\nabla \phi\|^{2} \Delta k\right] \mathrm{d} x+\frac{1}{2} \int_{\Omega}\|\nabla \phi\|^{2} \Delta k,
$$

donde temos

$$
\sum_{j=1}^{N} Q\left[\phi_{x_{j}}\right]=\frac{1}{2} \int_{\Omega} k(x) \Delta\left(\|\nabla \phi\|^{2}\right) \mathrm{d} x \leq 0
$$

Exemplo 1.7.1 Como um primeiro exemplo temos as equações com coeficientes de difusividade constantes constantes onde se tem $I_{2}=I_{3}=0$ e, quando o domínio $\Omega$ é convexo, pelo Teorema 1.2.1, $I_{1} \leq 0$.

Observação 1.7.1 Existem equações com coeficientes de difusão variáveis que admitem equilíbrios não constantes estáveis, independentemente da convexidade do domínio $\Omega$ conforme Nascimento [35]. Nesses casos o autor constrói uma função difusividade $k$ : $\Omega \longrightarrow \mathbb{R}$ de modo que, para uma classe particular de não-linearidades $f$, o problema admite equilíbrio não-constante estável. Entretanto, para esses exemplos notamos que $\Delta k$ é estritamente positivo numa região do domínio $\Omega$ o que, de certa forma, generaliza o resultado de Yanagida [42]. 


\subsection{Domínios Simétricos}

$\mathrm{Na}$ seção anterior consideramos equações definidas em domínios convexos $\Omega$ do espaço $\mathbb{R}^{N}$ e vimos que, sob as hipóteses admitidas, não foi possível determinar uma classe de funções difusividade $k$ para as quais fosse possível generalizar o resultado sobre a instabilidade de equilíbrios não-constantes de Matano [33] e Casten e Holland [7] uma vez que as únicas funções que satisfaziam as condições admitidas eram as funções constantes. Se, em lugar da convexidade, admitirmos alguma simetria dos domínios, então, sob hipóteses adequadas sobre a função difusividade, podemos obter um resultado análogo ao dos autores citados. Para as equações com coeficientes constantes, há um resultado análogo em Matano [33] e em Lopes [31].

Vamos considerar domínios invariantes por ação de elementos de $S O(N)$. Indiquemos por $R_{\theta} \in S O(N), \theta \in \mathbb{R}$ a aplicação sobre $\mathbb{R}^{N}$ que, em relação à base canônica, é dada pela matriz

$$
R_{\theta}=\left(\begin{array}{cccc}
\cos \theta & \operatorname{sen} \theta & \mid & 0 \\
\operatorname{sen} \theta & \cos \theta & \mid \\
-- & -- & -- \\
0 & \mid & I_{N-2}
\end{array}\right) .
$$

Diremos que $\Omega \subset \mathbb{R}^{N}$ é invariante por $R_{\theta}$, ou que é $R_{\theta}$-invariante, se $R_{\theta}(\Omega)=\Omega$.

Quando $\Omega$ é invariante por $R_{\theta}$ dizemos que $\phi: \Omega \rightarrow \mathbb{R}$ é invariante por $R_{\theta}$ se $\phi\left(R_{\theta} x\right)=\phi(x)$ para todo $x \in \Omega$.

Quando o domínio $\Omega$ for $R_{\theta}$-invariante poderemos considerar o sistema de coordenadas cilíndricas $\Psi:(0, \infty) \times(0,2 \pi) \times \mathbb{R}^{N-2} \rightarrow \Omega$ em $\Omega$ dado por

$$
(r, \theta, \tilde{x}) \longmapsto \Psi(r, \theta, \tilde{x})=(r \cos \theta, r \operatorname{sen} \theta, \tilde{x})=\left(x_{1}, x_{2}, \tilde{x}\right)
$$

onde $\tilde{x}=\left(x_{3}, \ldots, x_{N}\right)$. Se $N=2, \Psi$ é chamado de coordenadas polares.

Seja $\phi: \Omega \rightarrow \mathbb{R}, \phi \in \mathscr{C}^{2}(\Omega) \cap \mathscr{C}^{1}(\bar{\Omega})$, dada em coordenadas cartesianas por $\phi(x)=$ $\phi\left(x_{1}, x_{2}, \ldots, x_{N}\right)$. Indiquemos $\varphi:(0, \infty) \times(0,2 \pi) \times \mathbb{R}^{N-2} \rightarrow \Omega$ a composta $\varphi(y)=$ $\phi(\Psi(y))=\phi(x)$ onde $x=\Psi(y)$ com $y=(r, \theta, \tilde{x})$. Vemos que se $\phi$ é invariante por $R_{\theta}$, então a função $\varphi$ não depende de $\theta$, isto é, $\frac{\partial \varphi}{\partial \theta}=0$. Indiquemos por $\nabla \phi(x)=$ $\left(\phi_{x_{1}}, \phi_{x_{2}}, \ldots, \phi_{x_{N}}\right)$ o gradiente de $\phi$ nas coordenadas $x_{1}, x_{2}, \ldots, x_{N}$ de $\Omega$. Em relação às 
coordenadas cilíndricas temos $\varphi(y)=\phi(\Psi(y))=\phi(x)$ donde

$\nabla \phi(x)=\nabla \varphi(y)(D \Psi)^{-1}(y)=\left(\cos \theta \frac{\partial \varphi}{\partial r}-\frac{1}{r} \operatorname{sen} \theta \frac{\partial \varphi}{\partial \theta}, \operatorname{sen} \theta \frac{\partial \varphi}{\partial r}+\frac{1}{r} \cos \theta \frac{\partial \varphi}{\partial \theta}, \frac{\partial \varphi}{\partial x_{3}}, \ldots, \frac{\partial \varphi}{\partial x_{N}}\right)$

Se $\xi: \Omega \rightarrow \mathbb{R}$ é uma função diferenciável então indicamos

$$
\langle\nabla \phi(x), \nabla \xi(x)\rangle=\frac{1}{r^{2}} \frac{\partial \phi}{\partial \theta} \frac{\partial \xi}{\partial \theta}+\langle\tilde{\nabla} \varphi, \tilde{\nabla} \xi\rangle
$$

onde

$$
\begin{gathered}
\tilde{\nabla} \varphi=\left(\frac{\partial \varphi}{\partial r}, \frac{\partial \varphi}{\partial x_{3}}, \ldots, \frac{\partial \varphi}{\partial x_{N}}\right), \\
\|\nabla \phi\|^{2}=\frac{1}{r^{2}}\left(\frac{\partial \varphi}{\partial \theta}\right)^{2}+\|\tilde{\nabla} \varphi\|^{2}, \\
\Delta \phi=\frac{\partial^{2} \phi}{\partial x_{1}^{2}}+\frac{\partial^{2} \phi}{\partial x_{2}^{2}}+\cdots+\frac{\partial^{2} \phi}{\partial x_{N}^{2}} .
\end{gathered}
$$

Em coordenadas cilíndricas expressamos

$$
\Delta \phi=\frac{1}{r} \frac{\partial \varphi}{\partial r}+\frac{1}{r^{2}} \frac{\partial^{2} \varphi}{\partial \theta^{2}}+\tilde{\Delta} \varphi
$$

Vamos considerar que o domínio $\Omega$ seja $R_{\theta}$-invariante. Com a mudança de coordenadas a equação (1.34) passa a ser expressa em termos das coordenadas cilíndricas. Suponha que $\phi: \bar{\Omega} \rightarrow \mathbb{R}$ seja um equilíbrio do problema (1.34), isto é, $\operatorname{div}(k \nabla \phi)+f(\phi)=$ 0 em $\Omega$. Se a função $k$ é invariante por $R_{\theta}$ então,

$$
\begin{gathered}
\frac{\partial}{\partial \theta}(\operatorname{div}(k \nabla \phi))+f^{\prime}(\phi) \frac{\partial \phi}{\partial \theta}=0 \\
\operatorname{div}\left(k \nabla\left(\frac{\partial \phi}{\partial \theta}\right)\right)+f^{\prime}(\phi) \frac{\partial \phi}{\partial \theta}=0 .
\end{gathered}
$$

Portanto, podemos escrever

$$
\begin{aligned}
-f^{\prime}(\phi)\left(\frac{\partial \phi}{\partial \theta}\right)^{2} & =\frac{\partial \phi}{\partial \theta} \operatorname{div}\left(k \nabla\left(\frac{\partial \phi}{\partial \theta}\right)\right) \\
& =\operatorname{div}\left(\frac{\partial \phi}{\partial \theta} k \nabla\left(\frac{\partial \phi}{\partial \theta}\right)\right)-\left\langle k \nabla\left(\frac{\partial \phi}{\partial \theta}\right), \nabla\left(\frac{\partial \phi}{\partial \theta}\right)\right\rangle \\
& =\operatorname{div}\left(\frac{\partial \phi}{\partial \theta} k \nabla\left(\frac{\partial \phi}{\partial \theta}\right)\right)-k\left\|\nabla\left(\frac{\partial \phi}{\partial \theta}\right)\right\|^{2}
\end{aligned}
$$

donde segue que, quando $k$ é invariante por $R_{\theta}$ para toda $\phi: \Omega \rightarrow \mathbb{R}$ diferenciável vale

$$
Q\left[\frac{\partial \phi}{\partial \theta}\right]=\int_{\Omega}\left[k\left\|\nabla\left(\frac{\partial \phi}{\partial \theta}\right)\right\|^{2}-f^{\prime}(\phi)\left(\frac{\partial \phi}{\partial \theta}\right)^{2}\right]=\frac{1}{2} \int_{\partial \Omega} k \frac{\partial}{\partial \vec{n}}\left(\frac{\partial \phi}{\partial \theta}\right)^{2} .
$$


Lema 1.8.1 Seja $\Omega \subset \mathbb{R}^{N}$ invariante por $R_{\theta}$ com bordo $\partial \Omega$ suave. Se $\phi: \bar{\Omega} \rightarrow \mathbb{R}$ é uma função de classe $\mathscr{C}^{3}(\bar{\Omega})$ tal que $\frac{\partial \phi}{\partial \vec{n}}=0$ em $\partial \Omega$, então $\frac{\partial}{\partial \vec{n}}\left(\frac{\partial \phi}{\partial \theta}\right)=0$ em $\partial \Omega$.

Demonstração. Seja $h: \Omega_{\delta} \rightarrow \mathbb{R}$ de classe $\mathscr{C}^{3}$ invariante por $R_{\theta}$ tal que $\partial \Omega=h^{-1}(0)$ com $\nabla h(x) \neq 0$ para todo $x \in \partial \Omega$. Como a expressão $\langle\nabla \phi, \nabla h\rangle$ está definida para todo $x \in \bar{\Omega}$ seja $g: \bar{\Omega} \rightarrow \mathbb{R}$ de classe $\mathscr{C}^{1}$ tal que

$$
\langle\nabla \phi(x), \nabla h(x)\rangle=g(x) h(x), \forall x \in \bar{\Omega}
$$

Derivando em relação a $\theta$ e considerando que $h$ independe de $\theta$ vem

$$
\left\langle\nabla\left(\frac{\partial \phi}{\partial \theta}\right), \nabla h\right\rangle=\frac{\partial g}{\partial \theta} h
$$

Para $x \in \partial \Omega$ tem-se

$$
\left\langle\nabla\left(\frac{\partial \phi}{\partial \theta}\right), \vec{n}\right\rangle=\frac{\partial}{\vec{n}}\left(\frac{\partial \phi}{\partial \theta}\right)=0
$$

Teorema 1.8.1 Seja $\Omega \subset \mathbb{R}^{N}$ um domínio $R_{\theta}$-invariante com bordo $\partial \Omega$ suave e seja $\phi: \bar{\Omega} \rightarrow \mathbb{R}$ um equilibrio de (1.34) tal que $\phi$ não seja $R_{\theta}$-invariante. Se a função difusividade $k$ é $R_{\theta}$-invariante, então $\phi$ é instável.

Demonstração. Como $\phi$ não é $R_{\theta}$ invariante temos $\frac{\partial \phi}{\partial \theta} \not \equiv 0$ em $\Omega$. Como $k$ é $R_{\theta}$ invariante vale

$$
Q\left[\frac{\partial \phi}{\partial \theta}\right]=\frac{1}{2} \int_{\partial \Omega} k \frac{\partial}{\partial \vec{n}}\left(\frac{\partial \phi}{\partial \theta}\right) .
$$

Como $\phi$ é equilíbrio de (1.34) vale $\frac{\partial \phi}{\partial \vec{n}}=0$ em $\partial \Omega$. Pelo Lema 1.8.1 temos $\frac{\partial}{\partial \vec{n}}\left(\frac{\partial \phi}{\partial \theta}\right)=0$ em $\partial \Omega$, donde $Q\left[\frac{\partial \phi}{\partial \theta}\right]=0$.

Portanto, $\lambda_{1} \leq 0$. Se ocorresse $\lambda_{1}=0$, então $\frac{\partial \phi}{\partial \theta}$ seria uma autofunção do operador linear $\mathscr{L}$ em torno do equilíbrio $\phi$. Nesse caso, $\frac{\partial \phi}{\partial \theta}$ não trocaria de sinal em $\Omega$. Mas

$$
\int_{0}^{2 \pi} \frac{\partial \phi}{\partial \theta} \mathrm{d} \theta=0
$$

o que seria absurdo. Com isso, vem $\lambda_{1}<0$ implicando a instabilidade de $\phi$ como equilíbrio de (1.34). 
Proposição 1.8.1 Seja $\Omega$ um domínio $R_{\theta}$-invariante com bordo $\partial \Omega$ suave tal que, para todo $\theta$, a seção $\Omega_{\theta}$ seja convexa. Seja $\phi: \bar{\Omega}: \rightarrow \mathbb{R}$ de classe $\mathscr{C}^{2}(\Omega) \cap \mathscr{C}^{1}(\bar{\Omega})$ uma função $R_{\theta}$-invariante tal que $\frac{\partial \phi}{\partial \vec{n}}=0$ em $\partial \Omega$. Então,

$$
\frac{\partial}{\partial \vec{n}}\left(\|\nabla \phi\|^{2}\right) \leq 0 \text { em } \partial \Omega
$$

Demonstração. Considerando $h: \Omega_{\delta} \rightarrow \mathbb{R}$ tal que $\vec{n}=\frac{\nabla h}{\|\nabla h\|}$ para todo $x \in \partial \Omega=$ $h^{-1}(0)$ temos

$$
\frac{\partial}{\vec{n}}\left(\|\nabla \phi\|^{2}\right)=\left\langle\nabla\left(\|\nabla \phi\|^{2}\right), \frac{\nabla h}{\|\nabla h\|}\right\rangle
$$

Como $\phi$ e $h$ são $R_{\theta}$-invariantes temos que $\|\nabla \phi\|=\|\tilde{\nabla} \varphi\|$ e $\|\nabla h\|=\|\tilde{\nabla} h\|$ e, além disso, $\nabla\left(\|\nabla \phi\|^{2}\right)=\tilde{\nabla}\left(\|\tilde{\nabla} \varphi\|^{2}\right) \mathrm{e}$

$$
\frac{\partial}{\partial \vec{n}}\left(\|\nabla \phi\|^{2}\right)=\left\langle\tilde{\nabla}\left(\|\tilde{\nabla} \phi\|^{2}\right), \frac{\tilde{\nabla} h}{\|\tilde{\nabla} h\|}\right\rangle=\frac{\partial}{\partial \overrightarrow{\tilde{n}}}\left(\|\tilde{\nabla} \phi\|^{2}\right) \leq 0,
$$

pois, para cada $\theta$, a seção $\Omega_{\theta}$ é um subconjunto convexo.

\subsubsection{Domínios Anulares}

Um domínio $\Omega \subset \mathbb{R}^{N}$ é chamado anular se for determinado por duas esferas concêntricas, de centro na origem, isto é, $\Omega=\left\{x \in \mathbb{R}^{N} ; 0<r_{1} \leq\|x\| \leq r_{2}\right\}$. Como se percebe este é um tipo particular de domínio invariante por $R_{\theta}$. O bordo $\partial \Omega$ do domínio $\Omega$ é formado pelas esferas $S_{r_{1}}$ e $S_{r_{2}}$ onde $S_{r_{j}}=\left\{x \in \mathbb{R}^{N} ;\|x\|=r_{j}\right\}, j=1,2$. O vetor normal unitário exterior a $\Omega$ em $\partial \Omega$ é dado por $\vec{n}_{1}=-\frac{x}{r_{1}}$ para $x \in S_{r_{1}}$ e $\vec{n}_{2}=\frac{x}{r_{2}}$ para $x \in S_{r_{2}}$. Dizemos que $\phi: \bar{\Omega} \rightarrow \mathbb{R}$ de classe $\mathscr{C}^{2}(\Omega) \cap \mathscr{C}^{1}(\bar{\Omega})$ é uma função radial ou que é simétrica em relação à origem quando existe uma função $\omega:\left[r_{1}, r_{2}\right] \rightarrow \mathbb{R}, \omega \in \mathscr{C}^{2}\left(r_{1}, r_{2}\right) \cap \mathscr{C}^{1}\left(\left[r_{1}, r_{2}\right]\right)$ tal que

$$
\phi(x)=\omega(\|x\|)=\omega(r),
$$

onde $r=r(x)=\|x\|$ para todo $x \in \bar{\Omega}$. Nessas condições, temos

$$
\nabla \phi(x)=\omega^{\prime}(r) \nabla(\|x\|)=\omega^{\prime}(r) \frac{x}{\|x\|}, x \neq 0,
$$

donde segue $\|\nabla \phi(x)\|=\left|\omega^{\prime}(r)\right|$, com $r=\|x\|, x \in \bar{\Omega}$. Portanto, vale

$$
\nabla\left(\|\nabla \phi(x)\|^{2}\right)=\nabla\left(\left(\omega^{\prime}(r(x))\right)^{2}\right)=2 \omega^{\prime}(r(x)) \omega^{\prime \prime}(r(x)) \frac{x}{r} .
$$


Lema 1.8.2 Seja $\Omega \subset \mathbb{R}^{N}$ anular e seja $\phi: \bar{\Omega} \rightarrow \mathbb{R}$ radial, $\phi \in \mathscr{C}^{2}(\Omega) \cap \mathscr{C}^{1}(\bar{\Omega})$. Se $\frac{\partial \phi}{\partial \ddot{n}}=0$ em $\partial \Omega$, então

$$
\frac{\partial}{\partial \vec{n}}\|\nabla \phi\|^{2}=0, \text { em } \partial \Omega
$$

Demonstração. $\frac{\partial}{\partial \vec{n}}\left(\|\nabla \phi\|^{2}\right)=\left\langle\nabla\left(\|\nabla \phi\|^{2}\right), \vec{n}\right\rangle$ em $\partial \Omega$. De $\|\nabla \phi(x)\|=\left|\omega^{\prime}(r)\right|$ vem

$$
\nabla\left(\|\nabla \phi\|^{2}\right)=\nabla\left(\left(\omega^{\prime}(r)\right)^{2}\right)=2 \omega^{\prime}(r) \omega^{\prime \prime}(r) \frac{x}{r} .
$$

Como $\partial \Omega=S_{r_{1}} \cup S_{r_{2}}$, para $x \in S_{r_{1}}$ temos

$$
\frac{\partial}{\partial \vec{n}}\left(\|\nabla \phi\|^{2}\right)=2 \omega^{\prime}\left(r_{1}\right) \omega^{\prime \prime}\left(r_{1}\right)\left\langle\frac{x}{r_{1}}, \frac{-x}{r_{1}}\right\rangle=-2 \omega^{\prime}\left(r_{1}\right) \omega^{\prime \prime}\left(r_{1}\right) .
$$

Analogamente, para $x \in S_{r_{2}}$ temos

$$
\frac{\partial}{\partial \vec{n}}\left(\|\nabla \phi\|^{2}\right)=2 \omega^{\prime}\left(r_{2}\right) \omega^{\prime \prime}\left(r_{2}\right)
$$

Mas, $\frac{\partial \phi}{\partial \vec{n}}=\langle\nabla \phi, \vec{n}\rangle=\omega^{\prime}\left(r_{1}\right)\left\langle\frac{x}{r_{1}}, \frac{-x}{r_{1}}\right\rangle=-\omega^{\prime}\left(r_{1}\right)=0$ para todo $x \in S_{r_{1}}$. Analogamente, $\omega^{\prime}\left(r_{2}\right)=\frac{\partial \phi}{\partial \vec{n}}=0$ para todo $x \in S_{r_{2}}$, donde segue o resultado.

Pelo Lema 1.8.2 temos que a primeira integral da expressão (1.36) é nula, isto é, $\frac{1}{2} \int_{\partial \Omega} k \frac{\partial}{\partial \vec{n}}\left(\|\nabla \phi\|^{2}\right)=0$.

Lema 1.8.3 Seja $\Omega \subset \mathbb{R}^{N}$ anular e seja $\phi: \bar{\Omega} \rightarrow \mathbb{R}$ radial, $\phi \in \mathscr{C}^{2}(\Omega) \cap \mathscr{C}^{1}(\bar{\Omega})$. Se $\frac{\partial \phi}{\partial \vec{n}}=0$ em $\partial \Omega$, então

$$
\|\nabla \phi\|^{2}=0, \text { em } \partial \Omega
$$

Demonstração. Basta notar que $\|\nabla \phi(x)\|=\left|\omega^{\prime}(r)\right|$ e, portanto, para $x \in S_{r_{j}}, j=1,2$ valem $\frac{\partial \phi}{\partial \vec{n}}=-\omega^{\prime}\left(r_{1}\right)=0$ para todo $x \in S_{r_{1}}$ e $\frac{\partial \phi}{\partial \vec{n}}=\omega^{\prime}\left(r_{2}\right)=0$ para todo $x \in S_{r_{2}}$ de modo que

$$
\int_{\partial \Omega}\|\nabla \phi\|^{2} \frac{\partial k}{\partial \vec{n}}=\int_{S_{r_{1}}}\|\nabla \phi\|^{2} \frac{\partial k}{\partial \vec{n}}-\int_{S_{r_{2}}}\|\nabla \phi\|^{2} \frac{\partial k}{\partial \vec{n}}=0 .
$$

Como conseqüência imediata do Lema 1.8.3 temos que, se $\phi$ é um equilíbrio de (1.34), então a segunda integral da expressão (1.36) é nula. Podemos, então, enunciar um resultados para domínios anulares em termos que não foi possível enunciar para os domínios convexos. 
Teorema 1.8.2 Seja $\Omega$ domínio radial e seja $\phi \in \mathscr{C}^{2}(\Omega) \cap \mathscr{C}^{1}(\bar{\Omega})$ é um equilíbrio radial de (1.34). Se $k: \bar{\Omega} \rightarrow \mathbb{R}$ é uma função harmônica (super-harmônica) radial, então $\phi$ é instável.

Demonstração. Se $\phi$ é um equilíbrio de (1.34) então vale a condição de fronteira $\frac{\partial \phi}{\partial \vec{n}}=0$ em $\partial \Omega$. Pelos Lemas 1.8.2 e 1.8.3 temos que as duas primeiras integrais da expressão (1.36) são nulas. Como $k$ é uma função harmônica (super-harmônica) em $\Omega$, a terceira integral satisfaz $\int_{\Omega}\|\nabla \phi\|^{2} \Delta k \leq 0$, donde segue que $\sum_{j=1}^{N} Q\left[\phi_{x_{j}}\right] \leq 0$. Desta desigualdade segue a instabilidade de $\phi$ como equilíbrio de (1.34).

Observamos que se $k: \bar{\Omega} \rightarrow \mathbb{R}$ é uma função harmônica (super-harmônica) radial, então temos $k(x)=\xi(\|x\|)=\xi(r)$ onde $r=\|x\|$ para todo $x \in \bar{\Omega}$. Nesse caso, $\Delta k=0$ em $\Omega$ implica

$$
\Delta k=\sum_{j=1}^{N} k_{x_{j} x_{j}}=\xi^{\prime \prime}(r)+\frac{N-1}{r} \xi^{\prime}(r)=0 .
$$

Para $N=2$ a solução dessa equação é dada $\xi(r)=\log r$, donde $k(x)=\log \|x\|$. No caso $N \geq 3, k(x)=\|x\|^{2-N}$.

Em dimensão $N=2$ para a difusividade $k(x)=\log (\|x\|)$ o domínio anular $\Omega$ deve satisfazer $\Omega=\left\{x \in \mathbb{R}^{N} ; 1<r_{1}<\|x\|<r_{2}\right\}$ de modo que $k(x)>0 \forall x \in \Omega$.

Consideremos o caso em que $\Omega \subset \mathbb{R}^{N}$ é simétrico em relação ao hiperplano de equação $x_{k}=0$ onde $x=\left(x_{1}, \ldots, x_{k}, \ldots, x_{N}\right)$. Nessas condições vale um resultado análogo ao obtido por Lopes [31] para o caso de matriz com coeficientes variáveis, com uma propriedade de simetria. Indicamos por $\tilde{x}=\left(x_{1}, \ldots,-x_{k}, \ldots, x_{N}\right)$, onde $x \in \Omega$.

Teorema 1.8.3 Se a matriz $D=D(x)$ é uma matriz diagonal tal que $D(\tilde{x})=D(x)$ para todo $x \in \Omega$ e se $\phi$ é um equilibrio do problema (1.15) que é um mínimo global do funcional de energia (1.3), então $\phi(\tilde{x})=\phi(x)$ para todo $x \in \Omega$.

Demonstração. Seja $\phi=\phi(x), x \in \Omega$, um equilíbrio de (1.15) tal que $\phi$ seja um minimizante global do funcional de energia (1.3), aqui representado por

$$
E[u]=\int_{\Omega}\left(\frac{1}{2}\langle D(x) \nabla u, \nabla u\rangle-F(u(x))\right) \mathrm{d} x
$$

definido em $\mathbf{H}^{1}(\Omega) \cap \mathbf{L}^{\infty}(\Omega)$. Note que pelas hipóteses de crescimento e regularidade sobre $f$ o funcional está bem definido. Consideremos $\Omega_{d}=\left\{x \in \Omega ; x_{k} \geq 0\right\}$ e $\Omega_{e}=$ 
$\left\{x \in \Omega ; x_{k} \leq 0\right\}$, de modo que $\Omega=\Omega_{d} \cup \Omega_{e}$. Definamos $\xi: \Omega \longrightarrow \mathbb{R}$ por

$$
\xi(x)= \begin{cases}\phi(x), & \text { se } x \in \Omega_{r} \\ \phi(\tilde{x}), & \text { se } x \in \Omega_{l}\end{cases}
$$

é claro que $\xi \in \mathbf{H}^{1}(\Omega) \cap \mathbf{L}^{\infty}(\Omega)$. Portanto,

$$
\begin{gathered}
E[\xi]=\int_{\Omega}\left(\frac{1}{2}\langle D(x) \nabla \xi, \nabla \xi\rangle-F(\xi(x))\right) \mathrm{d} x \\
E[\xi]=\int_{\Omega_{d}}\left(\frac{1}{2}\langle D(x) \nabla \xi, \nabla \xi\rangle-F(\xi(x))\right) \mathrm{d} x+\int_{\Omega_{e}}\left(\frac{1}{2}\langle D(x) \nabla \xi, \nabla \xi\rangle-F(\xi(x))\right) \mathrm{d} x .
\end{gathered}
$$

Pelo Teorema de Mudança de Variáveis para as integrais múltiplas vale $E[\xi]=\int_{\Omega_{d}}\left(\frac{1}{2}\langle D(x) \nabla \phi, \nabla \phi\rangle-F(\phi(x))\right) \mathrm{d} x+\int_{\Omega_{d}}\left(\frac{1}{2}\left\langle D(\tilde{x}) I^{k} \nabla \phi(\tilde{x}), I^{k} \nabla \phi(\tilde{x})\right\rangle-F(\phi(\tilde{x}))\right) \mathrm{d} \tilde{x}$.

Sejam $E_{d}[\phi]=\int_{\Omega_{d}}\left(\frac{1}{2}\langle D(x) \nabla \phi, \nabla \phi\rangle-F(\phi(x))\right) \mathrm{d} x$ e $E_{e}[\phi]=\int_{\Omega_{e}}\left(\frac{1}{2}\langle D(x) \nabla \phi, \nabla \phi\rangle-\right.$ $F(\phi(x))) \mathrm{d} x$. Nessas condições, $E_{d}[\phi]=E_{e}[\phi]$ pois, se ocorresse $E_{d}[\phi]<E_{e}[\phi]$, teríamos

$$
E[\xi]=E_{d}[\phi]+E_{d}[\phi]<E_{d}[\phi]+E_{e}[\phi]=E[\phi]
$$

contrariando o fato de $\phi$ ser um minimizante global do funcional $E$. Da condição acima temos que $E[\phi]=E[\xi]$. Como $\xi$ também é um minimizante do funcional $E$ tem-se que $\xi$ é um equilíbrio de (1.15). Portanto, valem as equações

$$
\left\{\begin{aligned}
\operatorname{div}(D(x) \nabla \phi)+f(\phi) & =0, & & x \in \Omega \\
\operatorname{div}(D(x) \nabla \xi)+f(\xi) & =0, & & x \in \Omega \\
\frac{\partial \phi}{\partial \nu}=\frac{\partial \xi}{\partial \nu} & =0, & & x \in \partial \Omega .
\end{aligned}\right.
$$

Considerando $\eta(x)=\phi(x)-\xi(x), x \in \Omega$, temos

$$
\left\{\begin{aligned}
\operatorname{div}(D(x) \nabla \eta)+f^{\prime}(\phi) \eta & =0, & & x \in \Omega \\
\frac{\partial \eta}{\partial \nu} & =0, & & x \in \partial \Omega .
\end{aligned}\right.
$$

Observamos que $\eta=0$ é solução do sistema acima em $\Omega_{d}$. Como $\Omega$ é simétrico, pelo princípio da continuação, temos $\eta=0$ em $\Omega$. 
Corolário 1.8.1 Seja $\Omega$ uma bola centrada na origem ou um anel centrado na origem e seja a matriz diagonal $D=D(x)$ é simétrica em relação à origem. Se o equilibrio não-constante $\phi$ do sistema (1.15) também é simétrico em relação à origem, então é instável.

Pelo que vimos com os resultados acima parece que apenas a condição $\Delta k>0 \mathrm{em}$ um subconjunto de $\Omega$ ou domínio não-convexo não garantem a existência de equilíbrios não-constantes estáveis. São necessárias condições sobre a não linearidade conforme a proposição seguinte.

Proposição 1.8.2 No problema (1.1) se $f: \mathbb{R} \longrightarrow \mathbb{R}$ é uma função convexa de classe $\mathscr{C}^{2}$, então a equação não admite equilíbrio não-constante estável. O resultado também é válido se f for uma função côncava.

Demonstração. Suponhamos que $\phi: \Omega \longrightarrow \mathbb{R}$ seja um equilíbrio não-constante de (1.1) e seja $m=\min \{\phi(x) ; x \in \bar{\Omega}\}$. Então $\phi(x) \geq m$ para todo $x \in \bar{\Omega}$. Do funcional $Q$ definido em (1.9) temos

$$
\begin{aligned}
Q[\phi-m] & =\int_{\Omega}\left[k(x)\|\nabla(\phi-m)\|^{2}-f^{\prime}(\phi)(\phi-m)^{2}\right] \mathrm{d} x \\
& =\int_{\Omega}[\operatorname{div}((\phi-m) k \nabla(\phi-m))-(\phi-m) \operatorname{div}(k \nabla(\phi-m)) \\
& =\int_{\partial \Omega}(\phi-m) k \frac{\partial(\phi-m)}{\partial \nu} \mathrm{d} \sigma-\int_{\Omega}(\phi-m)\left[\operatorname{div}(k \nabla(\phi-m))+f^{\prime}(\phi)(\phi-m)\right] \\
& =\int_{\Omega}(\phi-m)\left[f(\phi)-f^{\prime}(\phi)(\phi-m)\right],
\end{aligned}
$$

uma vez que a condição de equilíbrio nos dá $\operatorname{div}(k \nabla \phi)=-f(\phi)$ em $\Omega$ e $\frac{\partial \phi}{\partial \nu}=0$ em $\partial \Omega$. Da condição de convexidade da função $f$ temos

$$
\frac{f(\phi)-f(m)}{\phi-m}<f^{\prime}(\phi)
$$

donde segue que $f(m)>f(\phi)-f^{\prime}(\phi)(\phi-m)$ e assim,

$$
Q[\phi-m]<\int_{\Omega} f(m)(\phi(x)-m) \mathrm{d} x .
$$

Vamos mostrar que $f(m) \leq 0$ donde resultará $Q[\phi-m]<0$. Seja $\bar{x} \in \bar{\Omega}$ tal que $\phi(\bar{x})=m$. Se $\bar{x} \in \Omega$ então $\nabla \phi(\bar{x})=0$ e $\Delta \phi(\bar{x}) \geq 0$. Portanto,

$$
\operatorname{div}(k(\bar{x}) \nabla \phi(\bar{x}))+f(\phi(\bar{x}))=0,
$$


donde $f(m)=-k(\bar{x}) \Delta \phi(\bar{x}) \leq 0$.

Se $\bar{x} \in \partial \Omega$ consideremos um sistema de coordenadas

$$
g: \Omega_{\epsilon}(\bar{x}) \longrightarrow \mathbb{R}^{N}
$$

definido numa vizinhança $\Omega_{\epsilon}(\bar{x})$ de $\bar{x}$ tal que $g(\bar{x})=(0,0), g\left(\partial \Omega \cap \Omega_{\epsilon}(\bar{x})\right) \subset \mathbb{R}^{N-1}$ com $g(x)=\left(\tilde{y}, y_{N}\right)$ onde $\tilde{y}=\left(y_{1}, \ldots, y_{N-1}\right)$.

Seja $h: U \longrightarrow \Omega_{\epsilon}(\bar{x})$ uma parametrização tal que $h(0,0)=\bar{x}, h(y)=h\left(\tilde{y}, y_{N}\right)=x$ e $\xi(y)=\phi \circ h(y)=\phi(x)$. Temos que $(0,0)$ é mínimo local de $\xi$

$$
\begin{aligned}
& \xi(0, s)=\xi(0,0)+\frac{\partial \xi}{\partial y_{N}}(0,0) s+\frac{1}{2} \frac{\partial^{2} \xi}{\partial y_{N}^{2}}(0,0) s^{2}+o\left(s^{3}\right) \\
& \xi(r, 0)=\xi(0,0)+\nabla_{\tilde{y}} \xi(0,0) \cdot r+\frac{1}{2} \frac{\partial^{2} \xi}{\partial \tilde{y}^{2}}(0,0)(r, r)+o\left(\|r\|^{3}\right)
\end{aligned}
$$

donde concluimos que $\frac{\partial^{2} \xi}{\partial y_{N}^{2}}(0,0) \geq 0$ e que $\frac{\partial^{2} \xi}{\partial \tilde{y}^{2}}(0,0)(r, r) \geq 0$ donde segue $f(m)=$ $-k(h(0)) \Delta \xi(h(0)) \leq 0$. 


\section{Capítulo 2}

\section{Sistemas Unidimensionais}

Neste capítulo vamos estudar os problemas de estabilidade de alguns sistemas com coeficientes variáveis definidos em intervalos reais. Uma razão para isso vem do fato de que nos casos de equações com coeficientes constantes em dimensão $N=1$ não há equilíbrio estável exceto, possivelmente, os equilíbrios constantes. Entretanto, para as equações com coeficientes variáveis é possível a existência de equilíbrios não-constantes estáveis conforme Yanagida [42]. Pretendemos analisar em que medida uma propriedades análogas são válidas para os sistemas de equações diferenciais parciais.

\subsection{Preliminares}

Nesta seção consideraremos os sistemas definidos em intervalos do tipo $\Omega=(0, l) \subset \mathbb{R}$, dados por

$$
\left\{\begin{aligned}
R(x) u_{t} & =\left(A(x) u_{x}\right)_{x}+f(u, v), 0<x<l, t>0 \\
S(x) v_{t} & =\left(B(x) v_{x}\right)_{x}+g(u, v), 0<x<l t>0 \\
u_{x}(0, t) & =u_{x}(l, t)=0=v_{x}(0, t)=v_{x}(l, t), t>0 .
\end{aligned}\right.
$$

onde $A, R: \Omega \longrightarrow \mathbb{M}_{m}(\mathbb{R})$ e $B, S: \Omega \longrightarrow \mathbb{M}_{n}(\mathbb{R})$ são funções de classe $\mathscr{C}^{2}$ com $A(x)=$ $\operatorname{diag}\left\{a_{1}(x), \ldots, a_{m}(x)\right\}, R(x)=\operatorname{diag}\left\{r_{1}(x), \ldots, r_{m}(x)\right\}$ e $B(x)=\operatorname{diag}\left\{b_{1}(x), \ldots, b_{n}(x)\right\}$, $S(x)=\operatorname{diag}\left\{s_{1}(x), \ldots, s_{n}(x)\right\}$ onde $a_{j}(x) \geq a_{0}>0, r_{j}(x) \geq r_{0}>0,1 \leq j \leq m$, e $b_{k}(x) \geq b_{0}>0, s_{k}(x) \geq s_{0}>0,1 \leq k \leq n$, para todo $x$ em $(0, l), f: \mathbb{R}^{m+n} \longrightarrow \mathbb{R}^{m} \mathrm{e}$ $g: \mathbb{R}^{m+n} \longrightarrow \mathbb{R}^{n}$ são funções de classe $\mathscr{C}^{1}$. 
Uma solução (clássica) do sistema (2.1) é um par de funções $(u, v)=(u(x, t), v(x, t))$ pertencentes a $\left(\mathscr{C}^{0}\left(\bar{\Omega} \times \mathbb{R}^{+} ; \mathbb{R}^{m}\right) \cap \mathscr{C}^{2}\left(\Omega \times \mathbb{R}^{+} ; \mathbb{R}^{m}\right)\right) \times\left(\mathscr{C}^{0}\left(\bar{\Omega} \times \mathbb{R}^{+} ; \mathbb{R}^{m}\right) \cap \mathscr{C}^{2}(\Omega \times\right.$ $\left.\left.\mathbb{R}^{+} ; \mathbb{R}^{m}\right)\right)$ satisfazendo as equações $(2.1)$ em $\Omega=(0, l)$ e as condições de fronteira estabelecidas.

Indicamos $u=\left(u_{1}, \ldots, u_{m}\right)^{T}$ e $v=\left(v_{1}, \ldots, v_{n}\right)^{T}$ onde $u_{j}, v_{k}:(0, l) \times(0, T) \longrightarrow \mathbb{R}$, $1 \leq j \leq m, 1 \leq k \leq n$.

Vamos admitir que sejam válidas as condições para a existência de um semi-grupo (analítico) definido pelo sistema dado. Esse sistema pode ser considerado como um problema de valor inicial num espaço de Banach $X$ dado por uma equação ordinária da forma $u_{t}=\mathbb{A} u+f(u)$ onde $\mathbb{A}$ é um operador setorial densamente definido em $X$ e $f: X \longrightarrow X$ localmente Lipschitz contínua. Pelas condições do problema dado consideramos $X=\left(\mathrm{L}^{2}(0,1)\right)^{(m+n)}, \mathbb{A}: D(\mathbb{A}) \subset X \rightarrow X$ dado por $\mathbb{A}[w]=-\left(\mathbb{D} w^{\prime}\right)^{\prime}$ onde $D(\mathbb{A})=\left(\mathbf{H}^{2}(0,1)\right)^{m} \times\left(\mathbf{H}^{2}(0,1)\right)^{n}, u^{\prime}(0)=u^{\prime}(l)=0$ e $v^{\prime}(0)=v^{\prime}(l)=0$ onde indicamos $\mathbb{D} w=(A u, B v)^{T}$.

Definição 2.1.1 Dizemos que o sistema (2.1) possui estrutura anti-gradiente ou que é um sistema anti-gradiente quando existe uma função $H: \mathbf{R}^{m+n} \longrightarrow \mathbf{R}$ de classe $\mathscr{C}^{2}$ tal que $f(u, v)=\nabla_{u} H(u, v)=\frac{\partial H}{\partial u}(u, v)$ e $g(u, v)=-\nabla_{v} H(u, v)=-\frac{\partial H}{\partial v}(u, v)$, onde $\nabla_{u} H(u, v)=\left(\frac{\partial H}{\partial u_{1}}, \ldots, \frac{\partial H}{\partial u_{m}}\right)^{T}$ e $\nabla_{v} H(u, v)=\left(\frac{\partial H}{\partial v_{1}}, \ldots, \frac{\partial H}{\partial v_{n}}\right)^{T}$.

Os sistemas de equações diferenciais parciais com estrutura anti-gradiente podem ser considerados como acoplamento anti-simétrico de dois sistemas do tipo gradiente o que permite o uso de algumas ferramentas mais usuais para o seu tratamento. Por analogia aos sistemas Hamiltonianos o sistema (2.1) se escreve como

$$
\left\{\begin{aligned}
R(x) u_{t} & =\left(A(x) u_{x}\right)_{x}+\nabla_{u} H(u, v), \quad 0<x<l, t>0 \\
S(x) v_{t} & =\left(B(x) v_{x}\right)_{x}-\nabla_{v} H(u, v), \quad 0<x<l, t>0 \\
u_{x}(0, t) & =u_{x}(l, t)=0=v_{x}(0, t)=v_{x}(l, t), t>0
\end{aligned}\right.
$$

Um dos exemplos de sistemas anti-gradiente a ser estudado com algum detalhe mais adiante é o bem conhecido sistema de equações de FitzHugh-Nagumo, ver Smoller [39], 
dado por

$$
\left\{\begin{aligned}
u_{t} & =u_{x x}+u(1-u)(u-a)-\epsilon v, 0<x<l, t>0, \\
v_{t} & =D v_{x x}+\epsilon(u-\gamma v), 0<x<l, t>0, \\
u_{x}(0, t) & =u_{x}(l, t)=0=v_{x}(0, t)=v_{x}(l, t), t>0,
\end{aligned}\right.
$$

onde $D>0,0<a<1, \varepsilon>0$ e $\gamma>0$ são constantes e $H(u, v)=F(u)-\frac{\epsilon}{2} u v+\frac{\epsilon \gamma}{2} v^{2}$ com $F(u)=\int_{0}^{u} f(z) \mathrm{d} z$.

Um outro exemplo bem conhecido de sistemas anti-gradiente é dado pelo sistema do tipo Predador-Presa, ver Smoller [39]:

$$
\left\{\begin{aligned}
u_{t} & =\alpha \Delta u+u M(u, v), \quad x \in \Omega, \quad t>0 \\
v_{t} & =\beta \Delta v+v N(u, v), \quad x \in \Omega, \quad t>0 \\
\frac{\partial u}{\partial n}(x, t) & =\frac{\partial v}{\partial n}(x, t), \quad x \in \partial \Omega, \quad t>0
\end{aligned}\right.
$$

onde $\alpha>0, \beta>0$ e $u \frac{\partial M}{\partial v}+v \frac{\partial N}{\partial u}=0$, para todo $u, v$.

Definição 2.1.2 Dizemos que o par de funções $(u, v)=(\phi, \psi): \Omega \longrightarrow \mathbb{R}^{m+n}$ é um equilíbrio do problema (2.1) se $\phi$ e $\psi$ forem funções de classe $\mathscr{C}^{2}$ em $(0, l)$ e de classe $\mathscr{C}^{1}$ em $[0, l]$ e são solução do seguinte sistema elíptico

$$
\left\{\begin{array}{c}
\left(A(x) \phi^{\prime}\right)^{\prime}+f(\phi, \psi)=0,0<x<l \\
\left(B(x) \psi^{\prime}\right)^{\prime}+g(\phi, \psi)=0,0<x<l \\
\phi^{\prime}(0)=\phi^{\prime}(l)=0=\psi^{\prime}(0)=\psi^{\prime}(l)
\end{array}\right.
$$

O estudo local da estabilidade será desenvolvido por meio da linearização do problema em torno do equilíbrio. Se $(u, v)=(\phi(x), \psi(x))$ é um equilíbrio do problema (2.1), então a linearização de (2.1) em relação a (em torno de) $(\phi, \psi)$ é dada pelo seguinte sistema:

$$
\left\{\begin{aligned}
R(x) U_{t} & =\left(A(x) U_{x}\right)_{x}+f_{u}(\phi, \psi) U+f_{v}(\phi, \psi) V, \quad 0<x<l, t>0 \\
S(x) V_{t} & =\left(B(x) V_{x}\right)_{x}+g_{u}(\phi, \psi) U+g_{v}(\phi, \psi) V, \quad 0<x<l, t>0 \\
U_{x}(0, t) & =U_{x}(l, t)=0=V_{x}(0, t)=V_{x}(l, t), \quad t>0
\end{aligned}\right.
$$

Associado ao sistema linearizado (2.3) podemos considerar o operador linear $\mathscr{L}$ : $\left(\mathbf{H}^{2}(0,1)\right)^{m} \times\left(\mathbf{H}^{2}(0,1)\right)^{n} \longrightarrow\left(\mathbf{L}^{2}(0,1)\right)^{(m+n)}$ definido por

$$
\mathscr{L}\left(\begin{array}{l}
u \\
v
\end{array}\right)=-\left(\begin{array}{c}
\left(A(x) u^{\prime}\right)^{\prime} \\
\left(B(x) v^{\prime}\right)^{\prime}
\end{array}\right)-\left(\begin{array}{ll}
f_{u}(\phi, \psi) & f_{v}(\phi, \psi) \\
g_{u}(\phi, \psi) & g_{v}(\phi, \psi)
\end{array}\right)\left(\begin{array}{l}
u \\
v
\end{array}\right),
$$


para todo $(u, v) \in\left(\mathbf{H}^{2}(0,1)\right)^{m} \times\left(\mathbf{H}^{2}(0,1)\right)^{n}$ satisfazendo $u_{x}(0)=u_{x}(l)=v_{x}(0)=$ $v_{x}(l)=0$. Notamos que $f_{u}, f_{v}, g_{u}$ e $g_{v}$ são matrizes tais que $f_{u}(\phi, \psi) \in \mathbb{M}_{m \times m}(\mathbb{R})$, $f_{v}(\phi, \psi) \in \mathbb{M}_{m \times n}(\mathbb{R}), g_{u}(\phi, \psi) \in \mathbb{M}_{n \times m}(\mathbb{R})$ e $g_{v}(\phi, \psi) \in \mathbb{M}_{n \times n}(\mathbb{R})$. Da estrutura antigradiente do sistema (2.1), temos que $f_{v}(\phi, \psi)^{T}=-g_{u}(\phi, \psi)$.

O problema de auto-valor para o operador $\mathscr{L}$ associado ao problema linearizado (2.3) com condição de Neumann homogênea na fronteira, expresso por

$$
\left\{\begin{aligned}
\mathscr{L}[w](x) & =\lambda w(x), \quad x \in(0, l) \\
w_{x}(t) & =0, \quad t=0, t=l
\end{aligned}\right.
$$

onde $w=(u, v)^{T}$.

A estabilidade do equilíbrio $(\phi, \psi)$ do sistema (2.1) será estudada considerando-se o problema de auto-valores (2.5). Como o operador $\mathscr{L}$, definido em (2.4), não é autoadjunto seu espectro pode conter autovalores complexos com parte imaginária não-nula.

Definição 2.1.3 Dizemos que o equilíbrio $(\phi, \psi)$ é linearmente estável se existir $\delta>0$ tal que, para todo autovalor $\lambda$ do operador linearizado $\mathscr{L}$, se tenha $\operatorname{Re}(\lambda)>\delta$. Por outro lado, o equilíbrio é chamado linearmente instável se existir $\delta>0$ e existir algum autovalor $\lambda$ tal que $\operatorname{Re}(\lambda)<-\delta$.

Usaremos a propriedade anti-gradiente do sistema (2.1) de maneira a obter algumas informações sobre o espectro do operador $\mathscr{L}$. As manipulações algébricas efetuadas a seguir têm por objetivo mostrar que é possível obter algumas informações sobre a estabilidade ou sobre a instabilidade dos equilíbrios do sistema (2.1) por meio de considerações sobre os sistemas parciais adequadamente definidos. Para tanto, seja $\lambda$ um auto-valor do operador linear (2.4), isto é, $\lambda \in \mathbf{C}$ é tal que o sistema

$$
\left\{\begin{array}{rll}
\lambda R(x) U & =-\left(A(x) U^{\prime}\right)^{\prime}-f_{u}(\phi, \psi) U-f_{v}(\phi, \psi) V, \quad 0<x<l \\
\lambda S(x) V & =-\left(B(x) V^{\prime}\right)^{\prime}-g_{u}(\phi, \psi) U-g_{v}(\phi, \psi) V, \quad 0<x<l \\
U^{\prime}(0) & =U^{\prime}(l)=0=V^{\prime}(0)=V^{\prime}(l) .
\end{array}\right.
$$

tem solução não-nula. Multiplicando escalarmente a primeira equação de (2.6) por $\bar{U}$ e a conjugada da segunda por $V$, respectivamente, obtemos:

$$
\begin{aligned}
\left\langle-\left(A(x) U^{\prime}\right)^{\prime}, \bar{U}\right\rangle-\left\langle f_{u}(\phi, \psi) U, \bar{U}\right\rangle-\left\langle f_{v}(\phi, \psi) V, \bar{U}\right\rangle & =\lambda\langle R(x) U, \bar{U}\rangle \\
\left\langle-\left(B(x) \bar{V}^{\prime}\right)^{\prime}, V\right\rangle-\left\langle g_{u}(\phi, \psi) \bar{U}, V\right\rangle-\left\langle g_{v}(\phi, \psi) \bar{V}, V\right\rangle & =\bar{\lambda}\langle S(x) \bar{V}, V\rangle .
\end{aligned}
$$


Considerando a estrutura anti-gradiente do sistema (2.1), temos que, em $(0, l)$, vale a seguinte equação $\left\langle g_{u}(\phi, \psi) \bar{V}, U\right\rangle+\left\langle f_{v}(\phi, \psi) V, \bar{U}\right\rangle=0$ donde, somando membro a membro as igualdades e integrando em $[0, l]$, temos

$$
\begin{array}{r}
\int_{0}^{l}\left(-\left\langle\left(A U^{\prime}\right)^{\prime}, \bar{U}\right\rangle-\left\langle f_{u} U, \bar{U}\right\rangle\right) \mathrm{d} x+\int_{0}^{l}\left(-\left\langle\left(B \bar{V}^{\prime}\right)^{\prime}, V\right\rangle-\left\langle g_{v} \bar{V}, V\right\rangle\right) \mathrm{d} x= \\
\lambda \int_{0}^{l}\langle R U, \bar{U}\rangle \mathrm{d} x+\bar{\lambda} \int_{0}^{l}\langle S \bar{V}, V\rangle \mathrm{d} x .
\end{array}
$$

Da condição de fronteira e da integração por partes, temos

$$
\int_{0}^{l}-\left\langle\left(A(x) U^{\prime}\right)^{\prime}, \bar{U}\right\rangle \mathrm{d} x=\int_{0}^{l}\langle A(x) U, \bar{U}\rangle \mathrm{d} x
$$

e

$$
\int_{0}^{l}-\left\langle\left(B(x) \bar{V}^{\prime}\right)^{\prime}, V\right\rangle \mathrm{d} x=\int_{0}^{l}\langle B(x) \bar{V}, V\rangle \mathrm{d} x .
$$

Substituindo estas igualdades na expressão acima temos

$$
\begin{aligned}
\lambda \int_{0}^{l}\langle R(x) U, \bar{U}\rangle \mathrm{d} x+\bar{\lambda} \int_{0}^{l}\langle S(x) \bar{V}, V\rangle \mathrm{d} x= \\
\quad \int_{0}^{l}\left(\langle A U, \bar{U}\rangle-\left\langle f_{u} U, \bar{U}\right\rangle\right) \mathrm{d} x+\int_{0}^{l}\left(\langle B \bar{V}, V\rangle-\left\langle g_{v} \bar{V}, V\right\rangle\right) \mathrm{d} x .
\end{aligned}
$$

A expressão (2.7) indica que, para localizar os autovalores do operador $\mathscr{L}$, podemos considerar separadamente dois problemas parciais sendo que, cada um deles, é um problema auto-adjunto.

Definindo os funcionais $I:\left(\mathbf{H}^{1}(0,1)\right)^{m} \longrightarrow \mathbb{R}$ e $J:\left(\mathbf{H}^{1}(0,1)\right)^{n} \longrightarrow \mathbb{R}$ por

$$
I[U]=\int_{0}^{l}\left(\left\langle A U^{\prime}, \bar{U}^{\prime}\right\rangle-\left\langle f_{u} U, \bar{U}\right\rangle\right) \mathrm{d} x, \quad \forall U \in\left(\mathbf{H}^{1}(0,1)\right)^{m},
$$

e

$$
J[V]=\int_{0}^{l}\left(\left\langle B \bar{V}^{\prime}, V^{\prime}\right\rangle-\left\langle g_{v} \bar{V}, V\right\rangle\right) \mathrm{d} x, \quad \forall V \in\left(\mathbf{H}^{1}(0,1)\right)^{n},
$$

podemos escrever (2.7) como

$$
\lambda \int_{0}^{l}\langle R U, \bar{U}\rangle \mathrm{d} x+\bar{\lambda} \int_{0}^{l}\langle S \bar{V}, V\rangle \mathrm{d} x=I[U]+J[V] .
$$

Se considerarmos que $\lambda=\alpha+i \beta$, com $\alpha$ e $\beta$ reais, então poderemos escrever

$$
\begin{aligned}
\lambda \int_{0}^{l}\langle R U, \bar{U}\rangle \mathrm{d} x+\bar{\lambda} \int_{0}^{l}\langle S \bar{V}, V\rangle \mathrm{d} x= & \alpha\left(\int_{0}^{l}\langle R U, \bar{U}\rangle \mathrm{d} x+\int_{0}^{l}\langle S \bar{V}, V\rangle \mathrm{d} x\right) \\
& +i \beta\left(\int_{0}^{l}\langle R U, \bar{U}\rangle \mathrm{d} x-\int_{0}^{l}\langle S \bar{V}, V\rangle \mathrm{d} x\right) .
\end{aligned}
$$


Da expressão acima e da igualdade (2.7) vem

$$
\operatorname{Re}(\lambda)\left(\int_{0}^{1}\langle\mathrm{RU}, \overline{\mathrm{U}}\rangle \mathrm{dx}+\int_{0}^{\mathrm{l}}\langle\mathrm{S} \overline{\mathrm{V}}, \mathrm{V}\rangle \mathrm{dx}\right)=\mathrm{I}[\mathrm{U}]+\mathrm{J}[\mathrm{V}] .
$$

Observamos que a expressão (2.7) e, conseqüentemente, a soma (2.10) são válidas quaisquer que sejam os autovalores do operador (2.4) e suas respectivas auto-funções. Além disso, estamos considerando as seguintes definições $\langle U, \bar{U}\rangle=\sum_{j=1}^{m} u_{j} \bar{u}_{j} \geq 0$, $\langle\bar{V}, V\rangle=\sum_{k=1}^{n} \bar{v}_{k} v_{k} \geq 0,\langle R U, \bar{U}\rangle=\sum_{j=1}^{m} r_{j} u_{j} \bar{u}_{j} \geq 0,\langle S \bar{V}, V\rangle=\sum_{k=1}^{n} s_{k} \bar{v}_{k} v_{k} \geq 0$. Assim, a análise da estabilidade do sistema (2.1) pode ser feita a partir das análises das estabilidades dos equilíbrios de dois sistemas auto-adjuntos que serão definidos oportunamente. A equação (2.7) indica que, para estudar o problema de autovalores do operador $\mathscr{L}$, podemos considerar problemas análogos separadamente para cada uma das incógnitas $U$ e $V$.

Seja o funcional quadrático $E$ definido em $\left(\mathbf{H}^{1}(0, l)\right)^{m} \times\left(\mathbf{H}^{1}(0, l)\right)^{n}$ por

$$
E[u, v]=\int_{0}^{l}\left(\frac{1}{2}\left\langle A u^{\prime}, u^{\prime}\right\rangle-\frac{1}{2}\left\langle B v^{\prime}, v^{\prime}\right\rangle-H(u, v)\right) \mathrm{d} x .
$$

Pelas hipóteses admitidas para a função $H$, o funcional (2.11 )é de classe $\mathscr{C}^{2}$ e, além disso, para cada par de funções $(\phi, \psi)$ de $\left(\mathbf{H}^{1}(0, l)\right)^{m} \times\left(\mathbf{H}^{1}(0, l)\right)^{n}$ valem as derivadas parciais dadas pelas seguintes expressões:

$$
\frac{\mathrm{d}}{\mathrm{d} u} E(\phi, \psi)[U]=\int_{0}^{l}\left(\left\langle A \phi^{\prime}, U^{\prime}\right\rangle-f(\phi, \psi) \cdot U\right) \mathrm{d} x
$$

e

$$
\frac{\mathrm{d}^{2}}{\mathrm{~d} u^{2}} E(\phi, \psi)[U, U]=\int_{0}^{l}\left(\left\langle A U^{\prime}, U^{\prime}\right\rangle-\left\langle f_{u}(\phi, \psi) \cdot U, U\right\rangle\right) \mathrm{d} x
$$

onde a derivada parcial $\frac{\mathrm{d}}{\mathrm{d} u} E(\phi, \psi)$ é um funcional linear definido em $\left(\mathbf{H}^{1}(0, l)\right)^{m}$ e a derivada parcial de segunda ordem $\frac{\mathrm{d}^{2}}{\mathrm{~d} u^{2}} E(\phi, \psi)$ define um funcional quadrático em $\left.\mathbf{H}^{1}(0, l)\right)^{m}$.

Analogamente, valem

$$
\frac{\mathrm{d}}{\mathrm{d} v} E(\phi, \psi)[V]=\int_{0}^{l}\left(-\left\langle B \psi^{\prime}, V^{\prime}\right\rangle+g(\phi, \psi) \cdot V\right) \mathrm{d} x
$$

e

$$
\frac{\mathrm{d}^{2}}{\mathrm{~d} v^{2}} E(\phi, \psi)[V, V]=\int_{0}^{l}\left(-\left\langle B V^{\prime}, V^{\prime}\right\rangle+\left\langle g_{v}(\phi, \psi) \cdot V, V\right\rangle\right) \mathrm{d} x
$$


para o funcional linear $\frac{\mathrm{d}}{\mathrm{d} v} E(\phi, \psi)$ e o funcional quadrático $\frac{\mathrm{d}^{2}}{\mathrm{~d} v^{2}} E(\phi, \psi)$ definidos em $\left(\mathbf{H}^{1}(0, l)\right)^{n}$. Os funcionais $I$ e $J$ definidos, respectivemente, em (2.8) e (2.9) satisfazem as condições

$$
I[U]=\frac{\mathrm{d}^{2}}{\mathrm{~d} u^{2}} E(\phi, \psi)[U, U], \quad \forall U \in\left(\mathbf{H}^{1}(0, l)\right)^{m}
$$

e

$$
-J[V]=\frac{\mathrm{d}^{2}}{\mathrm{~d} v^{2}} E(\phi, \psi)[V, V], \quad \forall V \in\left(\mathbf{H}^{1}(0, l)\right)^{n} .
$$

Os cálculos acima mostram que, abordado adequadamente, o problema da estabilidade de equilíbrios do sistema (2.1) pode ser reduzido às análises das estabilidades de dois problemas parciais correspondentes, conforme definição a seguir.

\subsection{Sistemas parciais}

Vamos analisar independentemente os sistemas parciais que compõem (2.1). Para isso consideremos $\left(\phi, \hat{p} \|_{i} i\right)$ um equilíbrio do problema (2.1) e fixemos, inicialmente, $v=\psi(x)$ na primeira equação, obtendo o seguinte sistema parcial

$$
\left\{\begin{array}{l}
R(x) u_{t}=\left(A(x) u_{x}\right)_{x}+f(u, \psi(x)), 0<x<l, t>0 \\
u_{x}(0, t)=u_{x}(l, t)=0 t>0
\end{array}\right.
$$

Obviamente $u=\phi(x)$ é um equilíbrio de (2.16). Além disso, considerando-se o funcional $E_{1}$ definido por

$$
E_{1}[u]=E[u, \psi]=\int_{0}^{l}\left(\frac{1}{2}\left\langle A u^{\prime}, u^{\prime}\right\rangle-\frac{1}{2}\left\langle B \psi^{\prime}, \psi^{\prime}\right\rangle-H(u, \psi)\right) \mathrm{d} x, \forall u \in\left(\mathbf{H}^{1}(0, l)\right)^{m},
$$

temos que $\phi$ é equilíbrio de (2.16) se, e somente se, é ponto crítico do funcional $E_{1}$, uma vez que é verdadeira a seguinte relação

$$
\frac{\mathrm{d}}{\mathrm{d} u} E_{1}(\phi)[U]=\int_{0}^{l}\left(\left\langle A \phi^{\prime}, U^{\prime}\right\rangle-f(\phi, \psi) \cdot U\right) \mathrm{d} x
$$

Se $u=u(x, t)$ é uma solução do sistema (2.16) então, considerando $E_{1}[u(., t)]$ como função diferenciável de $t$ temos

$$
\frac{\mathrm{d}}{\mathrm{d} t} E_{1}[u(t)]=-\int_{0}^{l}\left\langle R U^{\prime}, U^{\prime}\right\rangle \mathrm{d} x \leq 0,
$$

de modo que o sistema (2.16) representa o fluxo gradiente de $E_{1}$. 
Definição 2.2.1 Dizemos que a função $\phi \in\left(\mathbf{H}^{1}(0, l)\right)^{m}$ é um ponto de mínimo local para o funcional $E_{1}$ se existe $\delta>0$ tal que

$$
E_{1}[\phi] \leq E_{1}[\phi+u], \forall u \in\left(\mathbf{H}^{1}(0, l)\right)^{m}
$$

satisfazendo $\|u-\phi\|_{\mathbf{H}^{1}}<\delta$.

Notamos que se $\phi$ é um ponto de mínimo local do funcional $E_{1}$, então $\phi$ é um equilíbrio do sistema (2.16).

Em relação à análise da estabilidade linear do sistema (2.16) consideremos o sistema linearizado referente ao equilíbrio $\phi$ é dado por

$$
\left\{\begin{array}{l}
R(x) U_{t}=\left(A(x) U_{x}\right)_{x}+f_{u}(\phi(x), \psi(x)) U, 0<x<l, t>0 \\
U_{x}(0, t)=U_{x}(l, t)=0 t>0
\end{array}\right.
$$

Em correspondência, consideremos $\mathscr{A}$ o operador linear em torno do equilíbrio $\phi, \mathscr{A}$ : $\left(\mathbf{H}^{2}(0,1)\right)^{m} \longrightarrow\left(\mathbf{L}^{2}(0,1)\right)^{m}$, dado por

$$
\mathscr{A}[U]=-\left(A(x) U^{\prime}\right)^{\prime}-f_{u}(\phi, \psi) U, \quad \forall U \in\left(\mathbf{H}^{2}(0,1)\right)^{m}, U^{\prime}(0)=U^{\prime}(l)=0,
$$

e seja o problema de auto-valores associado

$$
\left\{\begin{array}{c}
-\left(A(x) U^{\prime}\right)^{\prime}-f_{u}(\phi, \psi) U=\lambda R(x) U \\
U^{\prime}(0)=U^{\prime}(1)=0
\end{array}\right.
$$

No sistema (2.16), a matriz $f_{u}$ é simétrica e o operador $\mathscr{A}$ é auto-adjunto, logo os seus auto-valores formam uma seqüência $\left(\lambda_{n}\right)_{n \in \mathbb{N}}$ de números reais com a propriedade $\lambda_{n} \rightarrow \infty$, quando $n \rightarrow \infty$. Além disso, o menor deles, o autovalor principal, ver Smoller [39], é dado por

$$
\lambda^{u}=\inf _{\substack{U \in\left(\mathbf{H}^{1}(0,1)\right)^{m} \\ U \neq 0}} \frac{\int_{0}^{l}\left(\left\langle A(x) U^{\prime}, U^{\prime}\right\rangle-\left\langle f_{u} U, U\right\rangle\right) \mathrm{d} x}{\int_{0}^{l}\langle R(x) U, U\rangle \mathrm{d} x} .
$$

Definição 2.2.2 Dizemos que $\phi$ é linearmente estável como um equilíbrio de (2.16) quando $\lambda^{u}>0$ e, quando $\lambda^{u}<0$, dizemos que $\phi$ é instável.

Observamos que $I[U]=\int_{0}^{l}\left(\left\langle A U^{\prime}, U^{\prime}\right\rangle-\left\langle f_{u}(\phi, \psi) U, U\right\rangle\right) \mathrm{d} x, \quad \forall U \in\left(\mathrm{H}^{1}(0,1)\right)^{m}$. Se $U$ satisfaz as condições de fronteira, então podemos re-escrever a expressão do funcional 
$I$ de modo que $I[U]=\int_{0}^{l}\left(-\left\langle\left(A U^{\prime}\right)^{\prime}, U\right\rangle-\left\langle f_{u}(\phi, \psi) U, U\right\rangle\right) \mathrm{d} x$, donde temos $I[U]=$ $\langle\mathscr{A}[U], U\rangle$. Nessas condições, se $U$ for uma auto-função normalizada do operador $\mathscr{A}$ associada a um autovalor $\lambda$, então $I[U]=\lambda$.

Essa observação, juntamente com a expressão (2.13), motiva a seguinte definição.

Definição 2.2.3 Dizemos que um equilíbrio $\phi$ do sistema (2.16) é não-degenerado se o operador linearizado correspondente $\mathscr{A}$, definido em (2.17), é invertível.

Proposição 2.2.1 Consideremos $(\phi, \psi)$ um equilíbrio do sistema (2.1) de modo que $\phi$ seja equilíbrio de (2.16). São verdadeiras as seguintes afirmações:

(a). $\phi$ é linearmente estável se, e somente se, é um mínimo local não-degenerado.

(b). Se $\phi$ é linearmente instável, então não é um minimo local do funcional $E_{1}$.

Demonstração. (a). Se $\phi$ é linearmente estável, então $\lambda^{u}>0$ onde $\lambda^{u}$ é o auto-valor principal do operador $\mathscr{A}$. Portanto, pela expressão $(2.20)$, para cada $U$ em $\left(\mathbf{H}^{1}(0, l)\right)^{m}$ tem-se

$$
0<\lambda^{u} \leq I[U]=\int_{0}^{l}\left(\left\langle A(x) U^{\prime}, U^{\prime}\right\rangle-\left\langle f_{u} U, U\right\rangle\right) \mathrm{d} x,
$$

donde segue que $\mathscr{A}$ é invertível. Da expressão (2.13) podemos escrever

$$
E_{1}[\phi+\delta U]-E_{1}[\phi]=\frac{\delta^{2}}{2} \frac{\mathrm{d}^{2}}{\mathrm{~d} u^{2}} E_{1}(\phi)[U]+o\left(\delta^{3}\right) \geq 0
$$

de onde se conclui que $\phi$ é mínimo local de $E_{1}$. Reciprocamente, se $\phi$ é um mínimo não-degenerado de $E_{1}$, então

$$
E_{1}[\phi+\delta U]-E_{1}[\phi]=\frac{\delta^{2}}{2} \frac{\mathrm{d}^{2}}{\mathrm{~d} u^{2}} E_{1}(\phi)[U]+o\left(\delta^{3}\right) \geq 0
$$

donde se tem $I[U] \geq 0$ para todo $U$. De (2.20) concluimos que $\lambda^{u} \geq 0$ e, como $\phi$ é não-degenerado, tem-se $\lambda^{u}>0$.

(b). Se $\phi$ é instável, então $\lambda^{u}<0$. Logo, existe $U$ em $\left(\mathbf{H}^{1}(0, l)\right)^{m}$ tal que $I[U]<0$. Nesse caso,

$$
E_{1}[\phi+\delta U]-E_{1}[\phi]=\frac{\delta^{2}}{2} \frac{\mathrm{d}^{2}}{\mathrm{~d} u^{2}} E_{1}(\phi)[U]+o\left(\delta^{3}\right) \leq 0
$$

e isso mostra que $\phi$ não pode ser mínimo local de (2.16). 
Proposição 2.2.2 Seja $(\phi, \psi)$ equilibrio de (2.1) de modo que $u=\phi$ seja um equilibrio de (2.16). Sejam $a_{j}^{\prime \prime}(x)<0,0<x<l$, para $1 \leq j \leq m$ e $\left\|f_{v}(u, v)\right\|$ suficientemente pequeno num conjunto limitado de $\mathbb{R}^{m+n}$. Se $\phi$ for não-constante, então $\phi$ será instável.

Demonstração. Sejam os equilíbrios $\phi=\left(\phi_{1}, \ldots, \phi_{m}\right)$ e $\psi=\left(\psi_{1}, \ldots, \psi_{n}\right)$ do sistema (2.1) de modo que $\phi$ seja equilíbrio de (2.16), isto é, vale

$$
\left(A(x) \phi^{\prime}\right)^{\prime}+f(\phi, \psi)=0, \forall x \in(0, l) .
$$

Portanto, para cada $j, 1 \leq j \leq m$, vale a seguinte equação

$$
\left(a_{j}(x) \phi_{j}^{\prime}\right)^{\prime}+f_{j}(\phi, \psi)=0, \quad \forall x \in(0, l) .
$$

Derivando essa equação, multiplicando o resultado por $\phi_{j}^{\prime}$ e integrando no intervalo $[0, l]$ obtemos

$$
\int_{0}^{l}\left(a_{j} \phi_{j}^{\prime}\right)^{\prime \prime} \phi_{j}^{\prime} \mathrm{d} x+\int_{0}^{l}\left[\sum_{k=1}^{m} \frac{\partial f_{j}}{\partial u_{k}}(\phi, \psi) \phi_{k}^{\prime} \phi_{j}^{\prime}+\sum_{l=1}^{n} \frac{\partial f_{j}}{\partial v_{l}}(\phi, \psi) \psi_{l}^{\prime} \phi_{j}^{\prime}\right] \mathrm{d} x=0 .
$$

Pela integração por partes e pelas condições de fronteira, a primeira parcela da soma em (2.21) pode ser escrita convenientemente como

$$
\int_{0}^{l}\left(a_{j} \phi_{j}^{\prime}\right)^{\prime \prime} \phi_{j}^{\prime} \mathrm{d} x=-\int_{0}^{l}\left(a_{j} \phi_{j}^{\prime}\right)^{\prime} \phi_{j}^{\prime \prime} \mathrm{d} x=-\int_{0}^{l} a_{j}^{\prime} \phi_{j}^{\prime} \phi_{j}^{\prime \prime}+a_{j}\left(\phi_{j}^{\prime \prime}\right)^{2} \mathrm{~d} x .
$$

Com mais uma integração por partes e usando as condições de fronteira temos

$$
\int_{0}^{l} a_{j}^{\prime} \phi_{j}^{\prime} \phi_{j}^{\prime \prime}=-\frac{1}{2} \int_{0}^{l} a_{j}^{\prime \prime}\left(\phi_{j}^{\prime}\right)^{2} \mathrm{~d} x
$$

Substituindo-se esses resultados na expressão (2.21) temos

$$
\frac{1}{2} \int_{0}^{l} a_{j}^{\prime \prime}\left(\phi^{\prime}\right)^{2} \mathrm{~d} x+\int_{0}^{l}\left[\sum_{l=1}^{n} \frac{\partial f_{j}}{\partial v_{l}}(\phi, \psi) \psi_{l}^{\prime} \phi_{j}^{\prime}\right] \mathrm{d} x=\int_{0}^{l}\left[a_{j}(x)\left(\phi_{j}^{\prime \prime}\right)^{2}-\sum_{k=1}^{m} \frac{\partial f_{j}}{\partial u_{k}}(\phi, \psi) \phi_{k}^{\prime} \phi_{j}^{\prime}\right] \mathrm{d} x .
$$

Somando membro a membro, para $1 \leq j \leq m$, os termos da igualdade (2.22), notamos que o segundo membro da soma obtida pode ser dado por

$$
\sum_{j=1}^{m} a_{j}\left(\phi_{j}^{\prime \prime}\right)^{2}-\sum_{j=1}^{m} \sum_{k=1}^{m} \frac{\partial f_{j}}{\partial u_{k}}(\phi, \psi) \phi_{j}^{\prime} \phi_{k}^{\prime}
$$


que é o integrando da expressão que define o funcional $I$. Portanto, podemos expressar

$$
I\left[\phi^{\prime}\right]=\int_{0}^{l}\left(\left\langle A(x) \phi^{\prime \prime}, \phi^{\prime \prime}\right\rangle-\left\langle f_{u}(\phi, \psi) \phi^{\prime}, \phi^{\prime}\right\rangle\right) \mathrm{d} x .
$$

A soma dos termos do primeiro membro da igualdade (2.22) pode ser indicado por

$$
\frac{1}{2} \int_{0}^{l} \sum_{j=1}^{m} a_{j}^{\prime \prime}\left(\phi_{j}^{\prime}\right)^{2} \mathrm{~d} x+\int_{0}^{l}\left[\sum_{j=1}^{m} \sum_{l=1}^{n} \frac{\partial f_{j}}{\partial v_{l}}(\phi, \psi) \phi_{j}^{\prime} \psi_{l}^{\prime}\right] \mathrm{d} x
$$

Como vale a igualdade $\sum_{j=1}^{m} \sum_{l=1}^{n} \frac{\partial f_{j}}{\partial v_{l}}(\phi, \psi) \phi_{j}^{\prime} \psi_{l}^{\prime}=\left\langle f_{v}(\phi, \psi) \psi^{\prime}, \phi^{\prime}\right\rangle$, podemos escrever

$$
I\left[\phi^{\prime}\right]=\int_{0}^{l} \sum_{j=1}^{m} a_{j}^{\prime \prime}\left(\phi_{j}^{\prime}\right)^{2} \mathrm{~d} x+\int_{0}^{l}\left\langle f_{v}(\phi, \psi) \psi^{\prime}, \phi^{\prime}\right\rangle \mathrm{d} x .
$$

Considerando que os equilíbrios $(\phi, \psi)$ do sistema (2.1) são funções com derivadas limitas temos

$$
\left|\left\langle f_{v}(\phi, \psi) \psi^{\prime}, \phi^{\prime}\right\rangle\right| \leq\left\|f _ { v } ( \phi , \psi ) \left|\left\|| | \phi ^ { \prime } \left|\left\|\mid \psi^{\prime}\right\|\right.\right.\right.\right.
$$

Dessa forma, se a função $f$ for tal que a matriz de sua derivada parcial $f_{v}(u, v)$ tiver norma $\left\|f_{v}(u, v)\right\|$ suficientemente pequena num conjunto limitado, então, pelas hipóteses, $a_{j}^{\prime \prime}<0$ em $(0, l)$ para todo $1 \leq j \leq m$, donde segue da expressão (2.23) que $I\left[\phi^{\prime}\right] \leq 0$. Desse fato, decorre a instabilidade da função $\phi$ como equilíbrio do sistema (2.16).

Analogamente ao que foi feito em relação ao sistema (2.16), podemos estudar o comportamento do sistema parcial na variável $v$ considerando o equilíbrio $(\phi, \psi)$ do sistema (2.1) e fixando $u=\phi(x)$ na segunda equação obtendo

$$
\left\{\begin{aligned}
S(x) v_{t} & =\left(B(x) v_{x}\right)_{x}+g(\phi(x), v), 0<x<l, t>0 \\
v_{x}(0, t) & =v_{x}(l, t)=0 t>0
\end{aligned}\right.
$$

Nas condições em que foi definido $v=\psi(x)$ é um equilíbrio do sistema (2.24). Além disso, considerando-se o funcional quadrático $E_{2}$ definido por

$$
E_{2}[u]=E[\phi, v]=\int_{0}^{l}\left(\frac{1}{2}\left\langle A \phi^{\prime}, \psi^{\prime}\right\rangle-\frac{1}{2}\left\langle B v^{\prime}, v^{\prime}\right\rangle-H(\phi, v)\right) \mathrm{d} x, \forall v \in\left(\mathbf{H}^{1}(0, l)\right)^{n}
$$

temos que $\psi$ é equilíbrio de (2.24) se, e somente se, é ponto crítico do funcional $E_{2}$. Essa correspondência decorre do seguite fato:

$$
\frac{\mathrm{d}}{\mathrm{d} v} E_{2}(\phi)[V]=\int_{0}^{l}\left(-\left\langle B \psi^{\prime}, V^{\prime}\right\rangle+g(\phi, \psi) \cdot V\right) \mathrm{d} x .
$$


Em especial, se $v=v(x, t)$ é uma solução do sistema (2.24), então, considerando-se $E_{2}[v(., t)]$ como função diferenciável de $t$ temos

$$
\frac{\mathrm{d}}{\mathrm{d} t} E_{2}[v(t)]=\int_{0}^{l}\left\langle S V^{\prime}, V^{\prime}\right\rangle \mathrm{d} x \geq 0
$$

de modo que o sistema (2.24) representa o fluxo gradiente de $-E_{2}$.

Definição 2.2.4 A função $\psi \in\left(\mathbf{H}^{1}(0, l)\right)^{n}$ é dita um máximo local para o funcional $E_{2}$ se existe $\delta>0$ tal que

$$
E_{2}[\psi] \geq E_{2}[\phi+v], \forall v \in\left(\mathbf{H}^{1}(0 . l)\right)^{n}
$$

satisfazendo $\|v-\psi\|_{\mathbf{H}^{1}}<\delta$.

Pelas contas efetuadas acima, se $\psi$ é um ponto de máximo local do funcional $E_{2}$, então é um equilíbrio do sistema (2.24).

Para o estudo da estabilidade linear do sistema (2.1) considerando o sistema linearizado referente ao equilíbrio, temos

$$
\left\{\begin{aligned}
S(x) V_{t} & =\left(B(x) V_{x}\right)_{x}+g_{v}(\phi(x), \psi(x)) V, 0<x<l, t>0 \\
V_{x}(0, t) & =V_{x}(l, t)=0 t>0
\end{aligned}\right.
$$

Seja, em correspondência, o operador linear em torno do equilíbrio $\psi, \mathscr{B}:\left(\mathbf{H}^{2}(0,1)\right)^{n} \longrightarrow$ $\left(\mathbf{L}^{2}(0,1)\right)^{n}$ definido por

$$
\mathscr{B}[V]=-\left(B(x) V^{\prime}\right)^{\prime}-g_{v}(\phi, \psi) V, \quad \forall V \in\left(\mathbf{H}^{2}(0,1)\right)^{n}, V^{\prime}(0)=V^{\prime}(l)=0,
$$

e consideremos o problema de auto-valores associado

$$
\left\{\begin{array}{c}
-\left(B(x) V^{\prime}\right)^{\prime}-g_{v}(\phi, \psi) V=\lambda S(x) V, x \in(0, l) ; \\
V^{\prime}(0)=V^{\prime}(l)=0 .
\end{array}\right.
$$

Como o sistema (2.27) é auto-adjunto os auto-valores do operador $\mathscr{B}$ são reais e formam uma seqüência $\left(\lambda_{n}\right)_{n \in \mathbb{N}}$ divergente para $+\infty$. Usando o método variacional, o autovalor principal é obtido por

$$
\lambda^{v}=\inf _{\substack{V \in\left(\mathbf{H}^{1}(0,1)\right)^{n} \\ V \neq 0}} \frac{\int_{0}^{l}\left(\langle B(x) V, V\rangle-\left\langle g_{v} V, V\right\rangle\right) \mathrm{d} x}{\int_{0}^{l}\langle S(x) V, V\rangle \mathrm{d} x} .
$$


Definição 2.2.5 Dizemos que $\psi$ é estável como equilíbrio de (2.24) quando $\lambda^{v}>0$. Se $\lambda^{v}<0$ dizemos que $\psi$ é instável.

Para o caso do sistema (2.24), valem resultados análogos aos enunciados nas Proposições 2.2.1 e 2.2.2 com demonstrações análogas, com as devidas adaptações. Explicitamente, valem

Proposição 2.2.3 Seja $(\phi, \psi)$ um equilibrio do sistema (2.1) e considere $\phi$ seja equilibrio de (2.24). São verdadeiras as seguintes afirmações:

(a). $\psi$ é linearmente estável se, e somente se, é um máximo local não-degenerado.

(b). Se $\psi$ é linearmente instável, então não é um máximo local do funcional $E_{2}$.

Proposição 2.2.4 Seja $(\phi(x), \psi(x))$ equilíbrio de (2.1), de modo que $v=\psi$ seja um equilibrio para (2.24). Se $b_{k}^{\prime \prime}(x)<0,1 \leq k \leq n$ e se $g_{u}(u, v)$ for uma matriz tal que sua norma $\left\|g_{u}(u, v)\right\|$ seja suficientemente pequena num determinado sub-conjunto limitado de $\mathbb{R}^{m+n}$, então, todo equilibrio não-constante $\psi$ de (2.24), se existir, será linearmente instável.

Demonstração. A demonstração é análoga à da Proposição 2.2.2, considerando-se que temos uma expressão para o funcional $J$ dada por

$$
J\left[\psi^{\prime}\right]=\int_{0}^{l} \sum_{k=1}^{n} b_{k}^{\prime \prime}\left(\psi_{k}^{\prime}\right)^{2} \mathrm{~d} x+\int_{0}^{l}\left\langle g_{u}(\phi, \psi) \phi^{\prime}, \psi^{\prime}\right\rangle \mathrm{d} x .
$$

\subsection{Aplicações}

Pelas discussões realizadas na seção anterior, pelas propriedades dos equilíbrios dos sistemas parciais (2.16) e (2.24) e tendo em vista a relação (2.7) podemos analisar o comportamento do equilíbrio do sistema (2.1) dado. Um resultado imediato é o

Teorema 2.3.1 Seja $(u, v)=(\phi(x), \psi(x))$ um equilíbrio do sistema (2.1) tal que $u=$ $\phi(x)$ seja estável como equilibrio de (2.16) e $v=\psi(x)$ seja estável como equilibrio de (2.24). Se (2.1) é um sistema anti-gradiente, então $(\phi(x), \psi(x))$ é estável. 
Demonstração. De fato, por hipótese temos $\lambda^{u}>0$ e $\lambda^{v}>0$ e, das expressões $(2.20)$ e (2.28) vem

$$
\begin{aligned}
& \lambda^{u} \int_{0}^{l}\langle R U, \bar{U}\rangle \mathrm{d} x \leq \int_{0}^{l}\left(\left\langle A U^{\prime}, \bar{U}^{\prime}\right\rangle-\left\langle f_{u} U, \bar{U}\right\rangle\right) \mathrm{d} x, \quad \forall U \in\left(\mathbf{H}^{1}(0, l)\right)^{m}, \\
& \lambda^{v} \int_{0}^{l}\langle S \bar{V}, V\rangle \mathrm{d} x \leq \int_{0}^{l}\left(\left\langle B \bar{V}^{\prime}, V^{\prime}\right\rangle-\left\langle g_{v} \bar{V}, V\right\rangle\right) \mathrm{d} x, \quad \forall V \in\left(\mathbf{H}^{1}(0, l)\right)^{n} .
\end{aligned}
$$

Se $(U, V)$ é uma auto-função qualquer do operador (2.4) associado ao autovalor $\lambda$ das desigualdades acima e da expressão (2.7) vem

$$
\begin{array}{r}
\lambda \int_{0}^{l}\langle R U, \bar{U}\rangle \mathrm{d} x+\bar{\lambda} \int_{0}^{l}\langle S \bar{V}, V\rangle \mathrm{d} x= \\
\int_{0}^{l}\left(\left\langle A U^{\prime}, \bar{U}^{\prime}\right\rangle-\left\langle f_{u} U, \bar{U}\right\rangle\right) \mathrm{d} x+\int_{0}^{l}\left(\left\langle B \bar{V}^{\prime}, V^{\prime}\right\rangle-\left\langle g_{v} \bar{V}, V\right\rangle\right) \mathrm{d} x \\
\geq \lambda^{u} \int_{0}^{l}\langle R U, \bar{U}\rangle \mathrm{d} x+\lambda^{v} \int_{0}^{l}\langle S \bar{V}, V\rangle \mathrm{d} x>0 .
\end{array}
$$

Donde, de (2.10), segue que

$$
\operatorname{Re}(\lambda)\left(\int_{0}^{l}\langle R U, \bar{U}\rangle \mathrm{d} x+\int_{0}^{l}\langle S \bar{V}, V\rangle \mathrm{d} x\right)>0 .
$$

Como $\langle R U, \bar{U}\rangle \geq 0$ e $\langle S \bar{V}, V\rangle \geq 0$, a desigualdade acima indica que a parte real de $\lambda$ é positiva. Como $\lambda$ é um autovalor arbitrário de $\mathscr{L}$, temos a estabilidade do equilíbrio $(\phi, \psi)$.

Exemplo 2.3.1 Sistemas de equações do tipo Predador-Presa com difusividade variáveis têm sido motivo de estudos recentes, ver Du e Hsu [13]. Seja, por exemplo, o seguinte sistema do tipo Predador-Presa dado por

$$
\left\{\begin{aligned}
u_{t} & =\left(a(x) u_{x}\right)_{x}+\alpha u(1-u)-\gamma u v^{2}, \quad 0 \leq x \leq l, t>0 \\
v_{t} & =\left(b(x) v_{x}\right)_{x}+\beta v(1-v)+\gamma v u^{2}, \quad 0 \leq x \leq l, t>0 \\
u_{x}(0, t) & =u_{x}(l, t)=v_{x}(0, t)=v_{x}(l, t)=0, t>0 .
\end{aligned}\right.
$$

onde $a, b:(0, l) \longrightarrow \mathbb{R}$ são funções de classe $\mathscr{C}^{2}$ estitamente positivas em $(0, l), \alpha>0$, $\beta>0$.

O sistema é anti-gradiente uma vez que $f(u, v)=\alpha u(1-u)-\gamma u v^{2}, g(u, v)=$ $\beta v(1-v)+\gamma v u^{2}$ e, considerando $H(u, v)=\frac{\alpha}{2} u^{2}-\frac{\alpha}{3} u^{3}-\frac{\gamma}{2} u^{2} v^{2}-\frac{\beta}{2} v^{2}+\frac{\beta}{3} v^{3}$ tem-se 
$\frac{\partial H}{\partial u}(u, v)=f(u, v)$ e $-\frac{\partial H}{\partial v}(u, v)=g(u, v)$. Os equilíbrios constantes são dados pelas soluções do sistema não linear

$$
\left\{\begin{aligned}
\alpha u(1-u)-\gamma u v^{2} & =0 \\
\beta v(1-v)+\gamma v u^{2} & =0
\end{aligned}\right.
$$

Por inspeção direta obtemos $(0,0),(0,1)$ e $(1,0)$ e, se $\beta>\gamma$, então existem somente essas soluções. Afirmamos que $(0,1)$ é estável como equilíbrio do sistema dado.

Seja $v=1$ fixado na primeira equação do sistema originando

$$
\left\{\begin{aligned}
u_{t} & =\left(a(x) u_{x}\right)_{x}+\alpha u(1-u)-\gamma u, \quad 0 \leq x \leq l, t>0 \\
u_{x}(0, t) & =u_{x}(l, t)=0, \quad t>0 .
\end{aligned}\right.
$$

Temos que $u=0$ é o equilíbrio a ser considerado. Para $f_{1}(u)=(\alpha-\gamma) u-\alpha u^{2}$ $f_{1}^{\prime}(0)=(\alpha-\gamma)$. Se $\alpha<\gamma$, então $u=0$ será estável.

Por outro lado, fazendo $u=0$ e substituindo na segunda equação obtemos

$$
\left\{\begin{aligned}
v_{t} & =\left(b(x) v_{x}\right)_{x}+\beta v(1-v) 0 \leq x \leq l, t>0 \\
v_{x}(0, t) & =v_{x}(l, t)=0, t>0
\end{aligned}\right.
$$

Neste caso $v=1$ é o equilíbrio que interessa analisar. Considerando $g_{1}(v)=\beta v(1-v)=$ $\beta v-\beta v^{2}$ tem-se $g_{1}^{\prime}(1)=-\beta<0$ donde segue que $v=1$ é estável como equilíbrio.

Pelo Teorema 2.3.1, $(0,1)$ é equilíbrio estável do sistema dado.

O Teorema 2.3.1 afirma que as estabilidades parciais implicam a estabilidade do sistema dado e esse é um fato bastante intuitivo. Entretanto, o conhecimento das instabilidades dos equilíbrios dos sistemas parciais (2.16) e (2.24) não implica imediatamente a instabilidade do sistema original (2.1). É exatamente esse o conteúdo da seguinte afirmação.

Teorema 2.3.2 Seja $(\phi, \psi)$ uma solução do sistema (2.2) de modo que, para quaisquer $R$ e $S$, o par $(\phi, \psi)$ seja um equilíbrio de (2.1), um sistema com estrutura anti-gradiente. Se, para uma matriz $R(x)$ fixada, a função $\phi$ for um equilíbrio instável do sistema parcial (2.16) correspondente, então a matriz $S=S(x)$ pode ser escolhida de modo que $(\phi, \psi)$ seja instável como equilibrio de (2.1).

Demonstração. Vamos mostrar que é possível encontrar $\epsilon>0$ tal que, escolhendo-se a matriz $S$ satisfazendo $\left\|S^{-1}\right\|<\epsilon$ existirá um autovalor $\lambda$ do problema (2.6) com parte 
real negativa, conforme Yanagida [43]. Por comodidade vamos re-escrever o sistema (2.6):

$$
\left\{\begin{aligned}
-\left(A(x) U^{\prime}\right)^{\prime}-f_{u}(\phi, \psi) U-f_{v}(\phi, \psi) V & =\lambda R(x) U, \quad 0<x<l, \\
-\left(B(x) V^{\prime}\right)^{\prime}-g_{u}(\phi, \psi) U-g_{v}(\phi, \psi) V & =\lambda S(x) V, \quad 0<x<l, \\
U^{\prime}(0)=U^{\prime}(1)=U^{\prime}(0)=U(l) & =0 .
\end{aligned}\right.
$$

Considerando os operadores $\mathscr{A}$ e $\mathscr{B}$ definidos, respectivamente, em (2.18) e (2.26), este sistema pode ser re-escrito como

$$
\left\{\begin{array}{rlrl}
\mathscr{A} U-f_{v}(\phi, \psi) V & =\lambda R U, & & 0<x<l, \\
\mathscr{B} V-g_{u}(\phi, \psi) U & =\lambda S V, & & 0<x<l, \\
U^{\prime}(0)=U^{\prime}(l) & =V^{\prime}(0)=V^{\prime}(l)=0 . &
\end{array}\right.
$$

Da segunda equação obtemos

$$
(\lambda S-\mathscr{B}) V=-g_{u} U
$$

De $S\left(\lambda I-S^{-1} \mathscr{B}\right)$ observamos que, se $\left\|S^{-1}\right\|$ for suficientemente pequeno, o operador $(\lambda S(x)-\mathscr{B})$ será invertível e teremos

$$
V=-(\lambda S-\mathscr{B})^{-1} g_{u} U
$$

que, substituindo na primeira equação, nos dá

$$
\mathscr{A} U+f_{v}(\lambda S-\mathscr{B})^{-1} g_{u} U=\lambda R U
$$

Temos assim um problema de determinação de autovalores para o perturbado do operador linear $\mathscr{A}$, conforme Kato [25]. Fixado o operador $\mathscr{A}$, consideremos a correspondência que associa a cada número complexo $\mu$ e cada matriz $S$ o operador $\mathscr{A}(\mu, S)$, dado por

$$
(\mu, S) \longmapsto \mathscr{A}(\mu, S)=\mathscr{A}+f_{v}(\lambda S-\mathscr{B})^{-1} g_{u}
$$

Como, por hipótese, $\phi$ é um equilíbrio instável de (2.16), temos que $\lambda^{u}<0$, donde existe $\delta>0$ tal que $\mathscr{U}_{\delta}=\left\{z \in \mathbf{C} ;\left|z-\lambda^{u}\right|<\delta\right\} \subset\{\lambda \in \mathbf{C} ; \operatorname{Re}(\lambda)<0\}$ e $\mathscr{U}_{\delta}$ não contém outro autovalor de $\mathscr{A}$. Restringindo a $\mu \in \mathscr{U}_{\delta}$ podemos considerar o operador dado por

$$
(\mu, S) \mapsto \mathscr{A}_{1}(\mu, S)
$$


Temos assim uma aplicação $\mathscr{A}_{1}: \mathscr{U}_{\delta} \times \mathscr{M}_{K}^{+} \longrightarrow \mathscr{L}\left(\left(\mathbf{H}^{1}(0, l)\right)^{m},\left(\mathbf{L}^{2}(0, l)\right)^{m}\right)$ definida por

$$
\mathscr{A}_{1}=\mathscr{A}_{1}(\lambda, S)=f_{v} S^{-1}\left(\lambda-S^{-1} \mathscr{B}\right)^{-1} g_{u},
$$

onde $\mathscr{M}_{K}^{+}$é o conjunto das matrizes positivas dadas por $S:[0, l] \longrightarrow \mathbb{M}_{m}(\mathbb{R})$ tais que, a cada $x \in(0, l)$, associa uma matriz positiva $\operatorname{com} \operatorname{det} S(x) \geq K>0$ para todo $x$ em $[0, l]$. Observamos que $\mathscr{A}_{1}$ é contínua e que vale

$$
\left\|\mathscr{A}_{1}\right\| \leq\left\|f _ { v } \left|\left\||| S^{-1}\left|\|\|\left(\lambda I-S^{-1} \mathscr{B}\right)^{-1}\right|\right\|\left\|g_{u}\right\| \leq C\left\|S^{-1}\right\|\right.\right.
$$

Como a constante $C$ é bem determinada vem que, existe $K>0$ suficientemente grande tal que, $\left\|S^{-1}\right\|<\frac{\varepsilon}{C}$. Portanto, é possível tomar $S$ de modo que se tenha $\left\|\mathscr{A}_{1}\right\|<\varepsilon$, para cada $\varepsilon>0$ dado. Consideremos o problema de autovalor do operador perturbado por $\mathscr{A}_{1}$

$$
\left(\mathscr{A}+\mathscr{A}_{1}(\mu, S)\right) u=\lambda(\mu, S) R u
$$

onde os autovalores $\lambda=\lambda(\mu, S)$, i.é., para cada $\lambda \in \mathscr{U}_{\delta}$ e cada função $S$ obtemos um autovalor $\lambda(\mu, S)$. Pela observação acima, para $S$ tal que $\left\|S^{-1}\right\|$ seja suficientemente pequeno, temos que $\mid \lambda\left(\mu, S-\lambda^{u} \mid<\delta\right.$. Escolhendo-se, então, uma matriz $S \in \mathscr{M}_{K}^{+}$tal que $\left\|S^{-1}\right\|$ seja suficientemente pequeno podemos considerar a aplicação $\lambda: \mathscr{U}_{\delta} \longrightarrow \mathscr{U}_{\delta}$ expressa pela correspondência

$$
\mu \longmapsto \lambda(\mu, S)=\lambda(\mu)
$$

$\forall \mu \in \mathscr{U}_{\delta}$. Pela continuidade das fuções $\mathscr{A}_{1}$ e $\lambda: \mathscr{U}_{\delta} \longrightarrow \mathscr{U}_{\delta}$ temos, do teorema de ponto fixo de Brouwer que, existe $\mu \in \mathscr{U}_{\delta}$ tal que $\lambda(\mu)=\mu$. Esse $\mu \in \mathscr{U}_{\delta}$ é um autovalor do problema perturbado $(2.30)$ e, como $\operatorname{Re}(\mu)<0$ e o resultado segue.

Teorema 2.3.3 Considere o sistema (2.1) com estrutura anti-gradiente e seja $(\phi, \psi)$ uma solução não-constante do sistema (2.2). Admita que $a_{j}^{\prime \prime}(x) \leq 0,1 \leq j \leq m e$ $b_{k}^{\prime \prime}(x) \leq 0,1 \leq k \leq n$, para cada $x \in(0, l)$. Então, $\lambda^{u}<0$ ou $\lambda^{v}<0$.

Demonstração. Como $(\phi, \psi)$ é não-constante, consideremos os funcionais $I$ e $J$ calculados em $\phi^{\prime}$ e em $\psi^{\prime}$, respectivamente. Pelas expressões (2.23) e (2.29) temos

$$
I\left[\phi^{\prime}\right]=\int_{0}^{l} \sum_{j=1}^{m} a_{j}^{\prime \prime}\left(\phi_{k}^{\prime}\right)^{2} \mathrm{~d} x+\int_{0}^{l}\left\langle\phi^{\prime}, f_{v}(\phi, \psi) \psi^{\prime}\right\rangle \mathrm{d} x
$$


e

$$
J\left[\psi^{\prime}\right]=\int_{0}^{l} \sum_{k=1}^{n} b_{k}^{\prime \prime}\left(\psi_{k}^{\prime}\right)^{2} \mathrm{~d} x+\int_{0}^{l}\left\langle g_{u}(\phi, \psi) \phi^{\prime}, \psi^{\prime}\right\rangle \mathrm{d} x .
$$

Da estrutura anti-gradiente do sistema (2.1) temos

$$
\left\langle\phi^{\prime}, f_{v}(\phi, \psi) \psi^{\prime}\right\rangle+\left\langle g_{u}(\phi, \psi) \phi^{\prime}, \psi^{\prime}\right\rangle=\left\langle f_{v}^{T}(\phi, \psi) \phi^{\prime}+g_{u}(\phi, \psi) \phi^{\prime}, \psi^{\prime}\right\rangle=0
$$

donde segue que

$$
I\left[\phi^{\prime}\right]+J\left[\psi^{\prime}\right]=\int_{0}^{l} \sum_{j=1}^{m} a_{j}^{\prime \prime}\left(\phi_{k}^{\prime}\right)^{2} \mathrm{~d} x+\int_{0}^{l} \sum_{k=1}^{n} b_{k}^{\prime \prime}\left(\psi_{k}^{\prime}\right)^{2} \mathrm{~d} x .
$$

Pela hipóteses, temos $I\left[\phi^{\prime}\right]+J\left[\psi^{\prime}\right] \leq 0$. Afirmamos que ou $\lambda^{u}<0$ ou $\lambda^{v}<0$ pois, caso contrário, teríamos $\lambda^{u} \geq 0$ e $\lambda^{v} \geq 0$ e, por conseqüência, $I\left[\phi^{\prime}\right] \geq 0$ e $J\left[\psi^{\prime}\right] \geq 0$. Nesse caso, da desigualdade acima, teríamos $I\left[\phi^{\prime}\right]=0$ e $J\left[\psi^{\prime}\right]=0$ donde seguiria que $\lambda^{u}=0$ e $\lambda^{v}=0$. Supondo que $\phi^{\prime} \neq 0$, então $I\left[\phi^{\prime}\right]=0$, donde $\phi^{\prime}$ seria uma autofunção do operador $\mathscr{A}$ associada ao autovalor $\lambda^{u}=0$, isto é, $\phi^{\prime}$ seria uma solução do sistema linear

$$
\left\{\begin{aligned}
(A(x) w)^{\prime}+f_{u}(\phi, \psi) w & =0, \quad 0<x<l, \\
w(0)=w(l) & =0 .
\end{aligned}\right.
$$

Neste caso, $\phi^{\prime}=0$, e isso seria uma contradição, o que prova o Teorema.

Corolário 2.3.1 Considere o sistema (2.1) com estrutura anti-gradiente e seja $(\phi, \psi)$ uma solução não-constante do sistema (2.2). Admita que $a_{j}^{\prime \prime}(x) \leq 0,1 \leq j \leq m e$ $b_{k}^{\prime \prime}(x) \leq 0,1 \leq k \leq n$, para cada $x \in(0, l)$. Então, existem matrizes $R$ e $S$ tais que $(\phi, \psi)$ é um equilíbrio não-constante instável do sistema (2.1).

Demonstração. A prova do Corolário é obtida combinando os Teoremas 2.3.3 e 2.3.2.

Observação 2.3.1 Na demonstração do Teorema 2.3.2 um fato fica evidente. Para os sistemas anti-gradientes as matrizes $f_{v}^{T}$ e $-g_{u}^{T}$ têm um papel de provocar um "deslocamento" dos espectros dos operadores lineares $\mathscr{A}$ e $\mathscr{B}$. 
No caso de sistemas anti-gradientes com acoplamento da forma

$$
\left\{\begin{aligned}
u_{t} & =\left(a(x) u_{x}\right)_{x}+f(u)-\epsilon v, \quad 0<x<l, t>0 \\
v_{t} & =\left(b(x) v_{x}\right)_{x}+\epsilon u+g(v), \quad 0<x<l, t>0 \\
u_{x}(0) & =u_{x}(l)=0, \quad t>0 \\
v_{x}(0) & =v_{x}(l)=0, \quad t>0 .
\end{aligned}\right.
$$

onde $f(0)=g(0)=0$ e $H(u, v)=F(u)-\epsilon u v-G(v)$ com $F(u)=\int_{0}^{u} f(s) \mathrm{d} s$ e $G(v)=$ $\int_{0}^{v} g(\xi) \mathrm{d} \xi$, para $\epsilon$ suficientemente pequeno, o espectro do sistema dado pode ser avaliado em termos dos espectros dos sistemas parciais. Uma conseqüência dessa propriedade dos sistemas anti-gradientes é a existência de sistemas equilíbrio não-constante estável conforme o Exemplo (2.3.2) enquanto que, para os sistemas gradientes, isso é impossível.

Embora o resultado sobre os sistemas gradientes seja bem conhecido vamos incluir uma versão para sistemas com coeficientes variáveis a fim de que o texto fique completo. Seja o sistema seguinte

$$
\left\{\begin{aligned}
w_{t} & =\left(A(x) w_{x}\right)_{x}+H(w), 0<x<l, t>0 \\
w_{x}(0) & =w_{x}(l)=0, t>0 .
\end{aligned}\right.
$$

onde $l>0, A:(0, l) \longrightarrow \mathbb{M}_{m}(\mathbb{R})$ é tal que, para $x \in(0, l)$, a matriz diagonal $A(x)=$ $\operatorname{diag}\left\{a_{1}(x), a_{2}(x), \ldots, a_{m}(x)\right\}$ satisfaz $a_{k}(x)>0, k=1,2, \ldots, m, \forall x \in \Omega$. O termo nãolinear $H: \mathbb{R}^{m} \longrightarrow \mathbb{R}^{m}$ é uma função que, a cada $w=\left(u_{1}, u_{2}, \ldots, u_{m}\right) \in \mathbb{R}^{m}$, associa $H(w)=\left(H_{1}(w), H_{2}(w), \ldots, H_{m}(w)\right)$ tal que existe uma função $h: \mathbb{R}^{m} \longrightarrow \mathbb{R}$ de classe $\mathscr{C}^{2}$ satisfazendo $H_{j}(w)=\frac{\partial h}{\partial u_{j}}(w), 1 \leq j \leq m, \forall w \in \mathbb{R}^{m}$.

Nessas condições vale o seguinte

Teorema 2.3.4 Se, para cada $j, 1 \leq j \leq m, a_{j}:(0, l) \longrightarrow \mathbb{R}$ é uma função de classe $\mathscr{C}^{2}$ tal que $a_{j}^{\prime \prime}(x) \leq 0$ para $x \in(0, l)$, então todo equilíbrio $\phi=\phi(x)$ não-constante de (2.31), se existir, será instável.

Demonstração. Consideremos o operador linear correspondente à linearização em torno do equilíbrio $\phi(x)$ dado por

$$
\mathscr{L}(W)=-\left(A(x) W_{x}\right)_{x}-H^{\prime}(\phi(x)) W, \quad W^{\prime}(0)=W^{\prime}(l)=0
$$

e vamos mostrar que existe um autovalor negativo de $\mathscr{L}$. 
Seja o funcional

$$
Q[W]=\int_{0}^{l}\left\{\left\langle A(x) W^{\prime}, W^{\prime}\right\rangle-\left\langle H^{\prime}(\phi) W, W\right\rangle\right\} \mathrm{d} x
$$

Considerando que o equilíbrio não-constante $\phi$ sejam a função $W(x)=\phi^{\prime}(x) \not \equiv 0$ e

$$
Q\left[\phi^{\prime}\right]=\int_{0}^{l}\left\{\left\langle A(x)\left(\phi^{\prime \prime}\right), \phi^{\prime \prime}\right\rangle-\left\langle H^{\prime}(\phi)\left(\phi^{\prime}\right), \phi^{\prime}\right\rangle\right\} \mathrm{d} x .
$$

Vamos mostrar que, com as hipóteses, $Q\left[\phi^{\prime}\right] \leq 0$. Da condição de equilíbrio vale

$$
\left(A(x) \phi^{\prime}\right)^{\prime}+H(\phi)=0, \quad \phi^{\prime}(0)=\phi^{\prime}(l)=0 .
$$

Derivando essa expressão, multiplicando (escalarmente) por $\phi^{\prime}$ e integrando no intervalo $(0, l)$ temos

$$
\int_{0}^{l}\left(\left\langle\left(A(x) \phi^{\prime}\right)^{\prime \prime}, \phi^{\prime}\right\rangle+\left\langle H^{\prime}(\phi) \phi^{\prime}, \phi^{\prime}\right\rangle\right) \mathrm{d} x=0 .
$$

Usando a integração por partes e as condições de $\phi$ na fronteira podemos escrever a primeira integral de (2.32) como

$$
\begin{gathered}
\int_{0}^{l}\left\langle\left(A(x) \phi^{\prime}\right)^{\prime \prime}, \phi^{\prime}\right\rangle \mathrm{d} x=-\int_{0}^{l}\left\langle\left(A(x) \phi^{\prime}\right)^{\prime}, \phi^{\prime \prime}\right\rangle \mathrm{d} x \\
\int_{0}^{l}\left\langle\left(A(x) \phi^{\prime}\right)^{\prime \prime}, \phi^{\prime}\right\rangle \mathrm{d} x=-\int_{0}^{l}\left\langle A^{\prime}(x) \phi^{\prime}, \phi^{\prime \prime}\right\rangle \mathrm{d} x-\int_{0}^{l}\left\langle A \phi^{\prime \prime}, \phi^{\prime \prime}\right\rangle \mathrm{d} x .
\end{gathered}
$$

Pela definição do produto interno, usando a integração por partes e as condições de fronteira podemos escrever

$$
-\int_{0}^{l}\left\langle A^{\prime}(x) \phi^{\prime}, \phi^{\prime \prime}\right\rangle \mathrm{d} x=-\int_{0}^{l} \sum_{j=1}^{m} a_{j}^{\prime} \phi_{j}^{\prime} \phi_{j}^{\prime \prime} \mathrm{d} x=-\frac{1}{2} \int_{0}^{l} \sum_{j=1}^{m} a_{j}^{\prime}\left(\phi_{j}^{\prime 2}\right)^{\prime} \mathrm{d} x .
$$

Uma última integração por partes nos dá

$$
-\int_{0}^{l}\left\langle A^{\prime}(x) \phi^{\prime}, \phi^{\prime \prime}\right\rangle \mathrm{d} x=\frac{1}{2} \int_{0}^{l} \sum_{j=1}^{m} a_{j}^{\prime \prime}\left(\phi_{j}^{\prime}\right)^{2} \mathrm{~d} x
$$

Substituindo esses resultados na expressão (2.32) temos

$$
Q\left[\phi^{\prime}\right]=\int_{0}^{l}\left(\left\langle A \phi^{\prime \prime}, \phi^{\prime \prime}\right\rangle+\left\langle H^{\prime}(\phi) \phi^{\prime}, \phi^{\prime}\right\rangle\right) \mathrm{d} x=\frac{1}{2} \sum_{j=1}^{m}\left(\int_{0}^{l} a_{j}^{\prime \prime}\left(\phi_{j}^{\prime}\right)^{2} \mathrm{~d} x\right) .
$$


Da hipótese $a_{j}^{\prime \prime}(x) \leq 0$ vem $Q\left[\phi^{\prime}\right] \leq 0$, donde segue o resultado.

O seguinte exemplo mostra a existência de sistemas anti-gradientes com coeficientes constantes e com equilíbrio não-constante estável o que, pelo Teorema 2.3.4, é impossível para os sistemas gradientes. Além desse fato, o exemplo mostra um sistema antigradiente no qual uma das equações parciais tem equilíbrio instável e, no entanto, o sistema dado tem o equilíbrio correspondente estável.

Exemplo 2.3.2 Considere o seguinte sistema anti-gradiente

$$
\left\{\begin{aligned}
u_{t} & =u_{x x}+5 u+10 v, 0<x<\pi, t>0 \\
v_{t} & =15 v_{x x}-10 u-10 v, 0<x<\pi, t>0 \\
u_{x}(0, t) & =u_{x}(\pi, t)=0=v_{x}(0, t)=v_{x}(\pi, t), t>0
\end{aligned}\right.
$$

Como o sistema (2.33) tem coeficientes constantes, podemos usar o método de expansão em autofunções $\phi_{n}(x)=\cos n x$ correspondentes aos autovalores $\lambda_{n}=n^{2}, n=0,1,2, \ldots$. do problema de valor de fronteira com condições de Neumann homogêneo

$$
\left\{\begin{array}{l}
w_{t}=w_{x x}, \quad 0<x<\pi, t>0 \\
w_{x}=w_{x}=0, t>0 .
\end{array}\right.
$$

Os autovalores do sistema (2.33) são obtidos por análise dos autovalores das matrizes $A_{n}$ onde, para cada $n$, temos

$$
A_{n}=\left(\begin{array}{cc}
5-n^{2} & 10 \\
-10 & -10-15 n^{2}
\end{array}\right)
$$

e, correspondentemente, os autovalores de $A_{n}$, indicados por

$$
\lambda_{n}=\frac{-\left(16 n^{2}+5\right) \pm \sqrt{\left(16 n^{2}+5\right)^{2}-4\left(15 n^{4}-65 n^{2}+50\right)}}{2}, n=0,1,2, \ldots
$$

O sistema (2.33) tem equilíbrio não-constante quando existe $n \geq 1$ tal que $\lambda_{n}=0$. Pela expressão de $\lambda_{n}$ devemos encontrar $n$ tal que

$$
15 n^{4}-65 n^{2}+50=0
$$

o que ocorre para $n=1$. Considerando que um autovetor da matriz $A_{1}$ associado ao autovalor $\lambda=0$ é $(-5,2)$ obtemos, assim, o equilíbrio não-constante $(\phi(x), \psi(x))=$ $(-5 \cos x, 2 \cos x)$ como uma solução não-constante do sistema (2.33). 
Fixando $v(x)=2 \cos x$ no sistema (2.33) temos

$$
\left\{\begin{aligned}
u_{t} & =u_{x x}+5 u+20 \cos x, 0<x<\pi, t>0, \\
u_{x}(0, t) & =u_{x}(\pi, t)=0, \quad t>0 .
\end{aligned}\right.
$$

para o qual $u(x)=-5 \cos x$ é equilíbrio. Como $f^{\prime}(-5 \cos x)=5$ é constante e positivo o autovalor principal do operador linear em torno do equilíbrio é negativo e, nesse caso, o equilíbrio é $u=-5 \cos x$ é instável.

Por outro lado, fixando $u(x)=-5 \cos x$ na segunda equação, temos

$$
\left\{\begin{aligned}
v_{t} & =15 v_{x x}-10 v+50 \cos x, 0<x<\pi, t>0 \\
v_{x}(0, t) & =v_{x}(\pi, t)=0 t>0
\end{aligned}\right.
$$

$v=2 \cos x$ é equilíbrio estável desse sistema. De fato, isso ocorre porque $g^{\prime}(2 \cos x)=$ -10 é constante negativa e o autovalor principal do operador linear correspondente é negativo.

Como o sistema (2.33) é linear com coeficientes constantes, o seu linearizado em torno do equilíbrio $(\phi, \psi)$ é o próprio sistema dado, de modo que a análise da estabilidade do equilíbrio $(0,0)$ é feita considerando-se os autovalores das mesmas matrizes $A_{n}$. Pela expressão para os autovalores $\lambda_{n}$ obtida acima, concluimos que $\lambda_{n} \leq 0$ para todo $n$, donde segue que $(0,0)$ é estável como equilíbrio do linearizado. Portanto, $(\phi, \psi)$ é estável como equilíbrio do sistema dado.

Observação 2.3.2 Note que no segundo sistema parcial do exemplo acima temos uma equação parabólica com coeficientes constantes que possui um equilíbrio não constante estável. Comparar com o caso tratado em Chafee [8].

Observação 2.3.3 Note que, no exemplo acima, se os coeficientes de difusão $a_{1}$ e $a_{2}$ fossem iguais, então o sistema não teria solução não-constante estável.

Para o caso de sistemas do tipo gradiente obtemos um resultado análogo ao obtido em Yanagida [42]. Consideremos o sistema gradiente seguinte

$$
\left\{\begin{aligned}
w_{t} & =\left(D(x) w_{x}\right)_{x}+H(w), 0<x<l, t>0 \\
w_{x}(0) & =w_{x}(l)=0, t>0 .
\end{aligned}\right.
$$

onde $l>0$, e $D:(0, l) \longrightarrow \mathbb{M}_{m}(\mathbb{R}), x \in(0, l)$, é a matriz diagonal múltiplo da identidade de ordem $m, D(x)=a^{2}(x) I_{m}$, com $a(x)>0 \forall x \in \Omega$. H: $\mathbb{R}^{m} \longrightarrow \mathbb{R}^{m}$ é uma função 
que a cada $w=\left(u_{1}, u_{2}, \ldots, u_{m}\right) \in \mathbb{R}^{m}$ associa $H(w)=\left(H_{1}(w), H_{2}(w), \ldots, H_{m}(w)\right)$ onde $H_{j}: \mathbb{R}^{m} \longrightarrow \mathbb{R}$ são funções de classe $\mathscr{C}^{1}$ tais que existe uma função $h: \mathbb{R}^{m} \longrightarrow \mathbb{R}$ de classe $\mathscr{C}^{2}$ tal que $H_{j}(w)=\frac{\partial h}{\partial u_{j}}(w), 1 \leq j \leq m$.

Teorema 2.3.5 Se a $: \Omega \longrightarrow \mathbb{R}$ é uma função de classe $\mathscr{C}^{2}$ tal que $a^{\prime \prime}(x) \leq 0$ para $x \in$ $(0, l)$, então todo equilíbrio $\Psi(x)=\left(\phi_{1}(x), \phi_{2}(x), \ldots, \phi_{m}(x)\right)$ não-constante de (2.34), se existir, será instável.

Demonstração. Consideremos o operador linear correspondente à linearização em torno do equilíbrio $\Psi(x)$ dado por

$$
\begin{gathered}
\mathscr{L}(W)=-\left(a^{2}(x) W_{x}\right)_{x}-H^{\prime}(\Psi(x)) W \\
\langle\mathscr{L} W, W\rangle=\int_{0}^{l}\left\{-\left\langle\left(a^{2}(x) W_{x}\right)_{x}, W\right\rangle-\left\langle H^{\prime}(\Psi(x)) W, W\right\rangle\right\} \mathrm{d} x .
\end{gathered}
$$

$\langle\mathscr{L} W, W\rangle=-\left.a^{2}(x)\left\langle W(x), W^{\prime}(x)\right\rangle\right|_{0} ^{l}+\int_{0}^{l}\left\{a^{2}(x)\left\|W_{x}\right\|^{2}-\left\langle H^{\prime}(\Psi(x)) W, W\right\rangle\right\} \mathrm{d} x$.

Seja o funcional

$$
Q[W]=\int_{0}^{l}\left\{a^{2}(x)\left\|W^{\prime}\right\|^{2}-\left\langle H^{\prime}(\Psi) W, W\right\rangle\right\} \mathrm{d} x
$$

Considerando que o equilíbrio $\Psi$ é não-constante para a função $W(x)=a(x) \Psi^{\prime}(x) \not \equiv$ 0 vale

$$
\begin{gathered}
Q\left[a \Psi^{\prime}\right]=\int_{0}^{l}\left\{a^{2}(x)\left\|\left(a \Psi^{\prime}\right)^{\prime}\right\|^{2}-\left\langle H^{\prime}(\Psi)\left(a \Psi^{\prime}\right), a \Psi^{\prime}\right\rangle\right\} \mathrm{d} x . \\
\left.Q\left[a \Psi^{\prime}\right]=\int_{0}^{l} a^{2} \| \Psi^{\prime}\right)^{\prime} \|^{2} \mathrm{~d} x-\int_{0}^{l}\left\langle H^{\prime}(\Psi) \Psi^{\prime}, a\left(a \Psi^{\prime}\right)\right\rangle \mathrm{d} x .
\end{gathered}
$$

Pela regra da cadeia podemos escrever $\frac{d}{d x} H(\Psi(x))=H^{\prime}(\Psi(x)) \cdot \Psi^{\prime}(x)$ e

$$
\int_{0}^{l}\left\langle H^{\prime}(\Psi) \Psi^{\prime}, a\left(a \Psi^{\prime}\right)\right\rangle \mathrm{d} x=\int_{0}^{l}\left\langle\frac{d}{d x} H(\Psi(x)), a\left(a \Psi^{\prime}\right)\right\rangle \mathrm{d} x .
$$

Pelas condições de fronteira, a integração por partes nos dá

$$
\int_{0}^{l}\left\langle H^{\prime}(\Psi) \Psi^{\prime}, a\left(a \Psi^{\prime}\right)\right\rangle \mathrm{d} x=-\int_{0}^{l}\left\langle H(\Psi),\left(a\left(a \Psi^{\prime}\right)\right)^{\prime}\right\rangle \mathrm{d} x=-\int_{0}^{l}\left\langle\left(a\left(a \Psi^{\prime}\right)\right)^{\prime},\left(a\left(a \Psi^{\prime}\right)\right)^{\prime}\right\rangle \mathrm{d} x .
$$


Desenvolvendo a expressão do integrando na última integral da igualdade acima temos

$$
\int_{0}^{l}\left\langle\left(a\left(a \Psi^{\prime}\right)\right)^{\prime},\left(a\left(a \Psi^{\prime}\right)\right)^{\prime}\right\rangle \mathrm{d} x=\int_{0}^{l}\left\{a^{2}\left\|\left(a \Psi^{\prime}\right)^{\prime}\right\|^{2}-2 a a^{\prime}\left\langle a \Psi^{\prime},\left(a \Psi^{\prime}\right)^{\prime}\right\rangle+\left(a^{\prime}\right)^{2}\left\|\left(a \Phi^{\prime}\right)\right\|^{2}\right\} .
$$

Substituindo na expressão 2.35 resulta

$$
Q\left[a \Psi^{\prime}\right]=-\int_{0}^{l} a a^{\prime}\left(\left\|a \Psi^{\prime}\right\|^{2}\right)^{\prime}+\left(a^{\prime}\right)^{2}\left\|a \Psi^{\prime}\right\|^{2} \mathrm{~d} x .
$$

Com mais uma integração por partes e usando as condições de fronteira temos

$$
\begin{gathered}
Q\left[a \Psi^{\prime}\right]=\int_{0}^{l}\left(a a^{\prime}\right)^{\prime}\left\|a \Psi^{\prime}\right\|^{2}-\left(a^{\prime}\right)^{2}\left\|a \Psi^{\prime}\right\|^{2} \mathrm{~d} x . \\
\therefore Q\left[a \Psi^{\prime}\right]=\int_{0}^{l} a a^{\prime \prime}\left\|a \Psi^{\prime}\right\|^{2} \mathrm{~d} x .
\end{gathered}
$$

Como $a^{\prime \prime}(x) \leq 0$ temos que $Q\left[a \Psi^{\prime}\right] \leq 0$, donde $\lambda_{0}<0$. Logo, $\Psi(x)$ é instável como equilíbrio do sistema (2.34).

O argumento usado na prova do Teorema 2.3.5 não vale para sistemas cujos coeficientes sejam dados por uma matriz diagonal da forma $D(x)=\operatorname{diag}\left\{a_{1}^{2}(x), \ldots, a_{m}^{2}(x)\right\}$, com $a_{j} \neq a_{k}$ se $j \neq k$, exceto se o sistema for desacoplado uma vez que não vale, em geral, a igualdade $H^{\prime}\left(D \Psi^{\prime}\right)=D H^{\prime} \Psi^{\prime}$ que foi usada na passagem que resultou a expressão (2.35).

Exemplo 2.3.3 Existe sistema anti-gradiente com coeficientes de difusividade variáveis que admite equilíbrio não-constante estável. De fato, sejam $a$ e $b$ funções definidas em $(0,1)$ tais que $a^{\prime \prime}\left(x_{0}\right)>0$ e $b^{\prime \prime}\left(x_{1}\right)$ para $x_{0}, x_{1} \in(0,1)$. De acordo com Yanagida [42] podemos determinar $\phi=\phi(x)$ e $\tilde{f}$ de modo que $\phi$ seja equilíbrio estável para o sistema:

$$
\left\{\begin{aligned}
r(x) u_{t} & =\left(a^{2}(x) u_{x}\right)_{x}+\tilde{f}(u), 0<x<1, t>0, \\
u_{x}(0, t) & =u_{x}(1, t)=0, t>0
\end{aligned}\right.
$$

e, analogamente, determinar funções $\psi=\psi(x)$ e $\tilde{g}$ de modo que $\psi$ seja equilíbrio estável para

$$
\left\{\begin{aligned}
s(x) v_{t} & =\left(b^{2}(x) v_{x}\right)_{x}+\tilde{g}(v), 0<x<1, t>0 \\
v_{x}(0, t) & =v_{x}(1, t)=0, \quad t>0
\end{aligned}\right.
$$


Portanto, $(\phi, \psi)$ é um equilíbrio do sistema (desacoplado)

$$
\left\{\begin{aligned}
r(x) u_{t} & =\left(a^{2}(x) u_{x}\right)_{x}+\tilde{f}(u), 0<x<1, t>0 \\
s(x) v_{t} & =\left(b^{2}(x) v_{x}\right)_{x}+\tilde{g}(v), 0<x<1, t>0 \\
u_{x}(0, t) & =u_{x}(1, t)=0, t>0 \\
v_{x}(0, t) & =v_{x}(1, t)=0, t>0
\end{aligned}\right.
$$

com estrutura anti-gradiente onde

$$
H(u, v)=\int \tilde{f}(u) d u-\int \tilde{g}(v) d v .
$$

Pelo Teorema (2.3.1) temos que $(\phi, \psi)$ é um equilíbrio não constante estável do sistema assim construído.

\subsection{As Equações de FitzHugh-Nagumo}

Um dos exemplos de sistemas anti-gradientes é o bem conhecido sistema de equações de FitzHugh-Nagumo, com coeficientes constantes, abordado por vários autores. Esse sistema possui propriedades de estabilidade correspondentes às propriedades de estabilidade de equilíbrios de uma equação de evolução associada. Mostraremos que algumas propriedades dos sistema com coeficientes variáveis também são herdadas da equação do calor correspondente. Pelo fato de $b(x) \equiv 0$ há, nesses sistemas, determinadas peculiaridades que revelam as características comuns entre os equilíbrios dos sistemas e os das equações correspondentes.

Vamos considerar o seguinte sistema anti-gradiente conhecido como equações de FitzHugh-Nagumo, ver Conley e Smoller[10] e Smoller [39]:

$$
\left\{\begin{aligned}
u_{t} & =\left(a^{2}(x) u_{x}\right)_{x}+f(u)-\epsilon v, 0<x<1, t>0 \\
v_{t} & =\epsilon(u-\gamma v), 0<x<1, t>0 \\
u_{x}(0, t) & =u_{x}(1, t)=0, t>0
\end{aligned}\right.
$$

onde $\epsilon, \gamma$ são constantes reais positivas e $f$ é uma função real definida por $f(u)=$ $u(1-u)(u-a)$ onde $0<a<\frac{1}{2}$. Para este caso temos

$$
H(u, v)=\int f(z) d z-\epsilon u v+\frac{\epsilon \gamma}{2} v^{2} .
$$




\subsubsection{Estabilidade}

Supondo que $(u, v)=(\phi(x), \psi(x))$ seja um equilíbrio do sistema (2.36) temos

$$
\left\{\begin{aligned}
\left(a^{2}(x) \phi^{\prime}\right)^{\prime}+f(\phi)-\epsilon \psi & =0,0<x<1 \\
\epsilon(\phi-\gamma \psi) & =0,0<x<1 \\
\phi^{\prime}(0)=\phi^{\prime}(1) & =0
\end{aligned}\right.
$$

Da segunda equação de (2.37) temos $\psi=\frac{1}{\gamma} \phi$ que, substituindo na primeira equação, nos dá

$$
\left\{\begin{aligned}
\left(a^{2}(x) \phi^{\prime}\right)^{\prime}+f(\phi)-\frac{\epsilon}{\gamma} \phi & =0,0<x<1 \\
\phi^{\prime}(0)=\phi^{\prime}(1) & =0 .
\end{aligned}\right.
$$

Por outro lado, se considerarmos a equação do calor associada ao problema (2.38) dada por

$$
\left\{\begin{aligned}
u_{t} & =\left(a^{2}(x) u_{x}\right)_{x}+f(u)-\frac{\epsilon}{\gamma} u, 0<x<1, t>0 \\
u_{x}(0, t) & =u_{x}(1, t)=0, t>0
\end{aligned}\right.
$$

veremos que, se $(u, v)=(\phi(x), \psi(x))$ for equilíbrio de $(2.36)$, então teremos que $\psi(x)=$ $\frac{1}{\gamma} \phi(x)$ e $u=\phi(x)$ será equilíbrio de (2.39). Reciprocamente, se $u=\phi(x)$ for equilíbrio de (2.39) então para $\psi(x)=\frac{1}{\gamma} \phi(x)$ o par $(\phi(x), \psi(x))$ será equilíbrio de (2.36). Portanto, poderemos analisar os equilíbrios de (2.36) a partir do estudo dos equilíbrios do problema (2.39).

Seja o linearizado de (2.36) referente ao equilíbrio $(\phi, \psi)$ dado por

$$
\left\{\begin{aligned}
u_{t} & =\left(a^{2}(x) u_{x}\right)_{x}+f^{\prime}(\phi) u-\epsilon v, \quad 0<x<1, t>0 \\
v_{t} & =\epsilon(u-\gamma v), \quad 0<x<1, t>0 \\
u_{x}(0, t) & =u_{x}(1, t)=0, t>0
\end{aligned}\right.
$$

Consideremos $\mathscr{L}: \mathbf{H}^{2}(0,1) \times \mathbf{H}^{2}(0,1) \longrightarrow \mathbf{L}^{2}(0,1)$, o operador linear associado ao problema (2.40), dado por

$$
\mathscr{L}\left(\begin{array}{l}
u \\
v
\end{array}\right)=-\left(\begin{array}{c}
\left(a^{2}(x) u^{\prime}\right)^{\prime} \\
0
\end{array}\right)-\left(\begin{array}{cc}
f^{\prime}(\phi) & -\epsilon \\
\epsilon & -\epsilon \gamma
\end{array}\right)\left(\begin{array}{l}
u \\
v
\end{array}\right)
$$

com as seguintes condições de fronteira $u^{\prime}(0)=u^{\prime}(1)=0$. Seja o problema de autovalor para o operador $\mathscr{L}$, definido em (2.41) com condição de Neumann homogênea na 
fronteira, expresso por

$$
\left\{\begin{aligned}
\mathscr{L}[w](x) & =\lambda w(x), \quad x \in(0,1) \\
w^{\prime}(x) & =0, \quad x=0, \quad x=1
\end{aligned}\right.
$$

onde $w=(u, v)^{t}$, com $(u, v) \in \mathbf{H}^{2}(0,1) \times \mathbf{H}^{2}(0,1)$.

O problema de auto-valores consiste em determinar $\lambda \in \mathbf{C}$ tal que o sistema

$$
\left\{\begin{aligned}
\left(a^{2}(x) u^{\prime}\right)^{\prime}+f^{\prime}(\phi) u-\epsilon v+\lambda u & =0,0<x<1 \\
\epsilon(u-\gamma v)+\lambda v & =0,0<x<1 \\
u^{\prime}(0)=u^{\prime}(1) & =0 .
\end{aligned}\right.
$$

tenha solução não-nula. Como $u \not \equiv 0$, da segunda equação de (2.42) segue que $\epsilon \gamma$ não é autovalor de $\mathscr{L}$ e, portanto, podemos fazer

$$
v=\frac{\epsilon}{\epsilon \gamma-\lambda} u
$$

Substituindo na primeira equação de (2.42) nos dá

$$
\left\{\begin{aligned}
\left(a^{2}(x) u^{\prime}\right)^{\prime}+f^{\prime}(\phi) u+\frac{\epsilon^{2}}{\lambda-\epsilon \gamma} u+\lambda u & =0,0<x<1, \\
u^{\prime}(0)=u^{\prime}(1) & =0 .
\end{aligned}\right.
$$

Por outro lado, em relação ao equilíbrio $u=\phi$ do problema (2.39), consideremos o sistema linearizado dado por:

$$
\left\{\begin{aligned}
u_{t} & =\left(a^{2}(x) u_{x}\right)_{x}+f^{\prime}(\phi) u-\frac{\epsilon}{\gamma} u, 0<x<1, \quad t>0 \\
u_{x}(0, t) & =u_{x}(1, t)=0, \quad t>0
\end{aligned}\right.
$$

Em correspondência ao sistema $(2.44)$, temos o operador linear $\tilde{\mathscr{A}}: \mathbf{H}^{2}(0,1) \longrightarrow \mathbf{L}^{2}(0,1)$ dado por

$$
\tilde{\mathscr{A}}[u]=-\left(a^{2}(x) u^{\prime}\right)^{\prime}-f^{\prime}(\phi) u+\frac{\epsilon}{\gamma} u, \quad u^{\prime}(0)=u^{\prime}(1)=0,
$$

associado ao qual consideramos o seguinte problema de auto-valores

$$
\left\{\begin{array}{c}
\left(a^{2}(x) u^{\prime}\right)^{\prime}+f^{\prime}(\phi) u-\frac{\epsilon}{\gamma} u+\tilde{\lambda} u=0,0<x<1, \\
u^{\prime}(0)=u^{\prime}(1)=0 .
\end{array}\right.
$$

Temos, portanto, a seguinte situação, se $(u, v)=(\phi(x), \psi(x))$ for equilíbrio de (2.36), então $u=\phi(x)$ será equilíbrio de (2.39) e, reciprocamente, se $u=\phi(x)$ for equilíbrio 
de (2.39) então, definindo $\psi(x)=\frac{1}{\gamma} \phi(x)$ teremos que $(\phi(x), \psi(x))$ será equilíbrio de (2.36). Portanto, poderemos estudar a estabilidade dos equilíbrios do sistema (2.36) relacionando-os com os equilíbrios de (2.39) e explorando as relações convenientemente estabelecidas entre os auto-valores dos operadores $\mathscr{L}$ e $\tilde{\mathscr{A}}$ dados, respectivemente, em (2.41) e (2.45).

Os autovalores do problema (2.46) formam uma seqüência não-decrescente de números reais $\left(\tilde{\lambda}_{n}\right)_{n \in \mathbb{N}} \subset \mathbb{R}$, com a propriedade $\tilde{\lambda}_{n} \longrightarrow \infty$ quando $n \longrightarrow \infty$.

Podemos re-escrever o sistema (2.43) como

$$
\left\{\begin{aligned}
\left(a^{2}(x) u^{\prime}\right)^{\prime}+f^{\prime}(\phi) u-\frac{\epsilon}{\gamma} u+\left(\frac{\epsilon}{\gamma} u+\frac{\epsilon^{2}}{\lambda-\epsilon \gamma} u+\lambda u\right) & =0,0<x<1, \\
u^{\prime}(0)=u^{\prime}(1) & =0 .
\end{aligned}\right.
$$

Comparando os sistemas (2.47) e (2.46) concluimos que, para todo $n \in \mathbb{N}$, vale a seguinte relação entre os autovalores dos operadores $\mathscr{L}$ e $\tilde{\mathscr{A}}$ :

$$
\lambda_{n}+\frac{\epsilon^{2}}{\lambda_{n}-\epsilon \gamma}+\frac{\epsilon}{\gamma}=\tilde{\lambda}_{n} .
$$

Podemos expressar $\lambda_{n}$ em função de $\tilde{\lambda}_{n}$ considerando que, para cada $n \in \mathbb{N}$, vale a equação,

$$
\lambda_{n}^{2}-\left(\tilde{\lambda}_{n}-\frac{\epsilon}{\gamma}+\epsilon \gamma\right) \lambda_{n}+\tilde{\lambda}_{n} \epsilon \gamma=0 .
$$

Portanto, para cada $n \in \mathbb{N}$, temos

$$
\lambda_{n}=\frac{\left(\tilde{\lambda}_{n}-\frac{\epsilon}{\gamma}+\epsilon \gamma\right) \pm \sqrt{\Delta_{n}}}{2}
$$

e

$$
\Delta_{n}=\left(\tilde{\lambda}_{n}-\frac{\epsilon}{\gamma}+\epsilon \gamma\right)^{2}-4 \tilde{\lambda}_{n} \epsilon \gamma
$$

Por outro lado, da expressão (2.50) para $\Delta_{n}$, observamos que

$$
\begin{aligned}
\Delta_{n} & =\left(\tilde{\lambda}_{n}-\frac{\epsilon}{\gamma}\right)^{2}+2\left(\tilde{\lambda}_{n}-\frac{\epsilon}{\gamma}\right) \epsilon \gamma+(\epsilon \gamma)^{2}-4 \tilde{\lambda}_{n} \epsilon \gamma \\
& =\left(\tilde{\lambda}_{n}-\frac{\epsilon}{\gamma}\right)^{2}+2\left(\tilde{\lambda}_{n}-\frac{\epsilon}{\gamma}\right) \epsilon \gamma+(\epsilon \gamma)^{2}-4\left(\tilde{\lambda}_{n}-\frac{\epsilon}{\gamma}+\frac{\epsilon}{\gamma}\right) \epsilon \gamma \\
& =\left(\tilde{\lambda}_{n}-\frac{\epsilon}{\gamma}\right)^{2}-2\left(\tilde{\lambda}_{n}-\frac{\epsilon}{\gamma}\right) \epsilon \gamma+(\epsilon \gamma)^{2}-4 \epsilon^{2}
\end{aligned}
$$

Portanto, obtemos a seguinte expressão alternativa para $\Delta_{n}$

$$
\Delta_{n}=\left(\tilde{\lambda}_{n}-\frac{\epsilon}{\gamma}-\epsilon \gamma\right)^{2}-4 \epsilon^{2}
$$


Da expressão (2.51) temos que se, $\tilde{\lambda}_{n} \rightarrow \infty$, então $\Delta_{n} \rightarrow \infty$ quando $n \rightarrow \infty$. Portanto, existe eventualmente, um número finito de índices $n$ para os quais se tem $\Delta_{n}<0$ e, assim existe, no máximo, um número finito de autovalores complexos do problema (2.42).

Cada autovalor $\tilde{\lambda}_{n}$ do problema (2.46) dá origem a dois autovalores $\lambda_{n}^{+}$e $\lambda_{n}^{-}$do problema (2.42), relacionados entre si e indicados, respectivamente, por

$$
\lambda_{n}^{+}=\frac{\left(\tilde{\lambda}_{n}-\frac{\epsilon}{\gamma}+\epsilon \gamma\right)+\sqrt{\Delta_{n}}}{2} \quad \text { e } \quad \lambda_{n}^{-}=\frac{\left(\tilde{\lambda}_{n}-\frac{\epsilon}{\gamma}+\epsilon \gamma\right)-\sqrt{\Delta_{n}}}{2} .
$$

Temos, em conseqüência, duas seqüências $\left(\lambda_{n}^{+}\right)_{n \in \mathbb{N}}$ e $\left(\lambda_{n}^{-}\right)_{n \in \mathbb{N}}$ formadas por autovalores de (2.42). Como $\tilde{\lambda}_{n} \rightarrow+\infty$ quando $n \rightarrow+\infty$ existe $n_{0} \in \mathbb{N}$ tal que, para todo inteiro $n \geq n_{0}$, vale $\left(\tilde{\lambda}_{n}-\epsilon \gamma-\frac{\epsilon}{\gamma}\right)^{2}-4 \epsilon^{2}=\Delta_{n}>0$. Nessas condições, para $n \geq m \geq n_{0}$ valem $\Delta_{n} \geq \Delta_{m} \geq 0$ e

$$
\lambda_{n}^{-}=\frac{\left(\tilde{\lambda}_{n}+\epsilon \gamma-\frac{\epsilon}{\gamma}\right)-\sqrt{\Delta_{n}}}{2} \leq \frac{\left(\tilde{\lambda}_{n}+\epsilon \gamma-\frac{\epsilon}{\gamma}\right)+\sqrt{\Delta_{n}}}{2}=\lambda_{n}^{+}
$$

Assim $\left(\lambda_{n}^{+}\right)_{n \geq 0} \subset \mathbb{C}$ é uma seqüência tal que, para $n \geq n_{0}, \lambda_{n}^{+} \in \mathbb{R}$, com $\lambda_{n}^{+} \rightarrow \infty$ quando $n \rightarrow \infty$.

Quanto à seqüência $\left(\lambda_{n}^{-}\right)_{n \in \mathbb{N}}$, de auto-valores em (2.52), temos

$$
\begin{gathered}
\lim _{n \rightarrow \infty} \lambda_{n}^{-}=\lim _{n \rightarrow \infty} \frac{\left(\tilde{\lambda}_{n}-\frac{\epsilon}{\gamma}+\epsilon \gamma\right)-\sqrt{\Delta_{n}}}{2} \\
=\lim _{n \rightarrow \infty}\left[\frac{2 \tilde{\lambda}_{n} \epsilon \gamma}{\left(\tilde{\lambda}_{n}-\frac{\epsilon}{\gamma}+\epsilon \gamma\right)+\sqrt{\Delta_{n}}}\right] \\
=\lim _{n \rightarrow \infty}\left[\frac{2\left(\tilde{\lambda}_{n}-\frac{\epsilon}{\gamma}+\epsilon \gamma\right) \epsilon \gamma+2 \epsilon \gamma\left(\frac{\epsilon}{\gamma}-\epsilon \gamma\right)}{\left(\tilde{\lambda}_{n}-\frac{\epsilon}{\gamma}+\epsilon \gamma\right)+\sqrt{\Delta_{n}}}\right] \\
=\lim _{n \rightarrow \infty}\left[\frac{2\left(\tilde{\lambda}_{n}-\frac{\epsilon}{\gamma}+\epsilon \gamma\right) \epsilon \gamma}{\left(\tilde{\lambda}_{n}-\frac{\epsilon}{\gamma}+\epsilon \gamma\right)+\sqrt{\Delta_{n}}}+\frac{2 \epsilon \gamma\left(\frac{\epsilon}{\gamma}-\epsilon \gamma\right)}{\left(\tilde{\lambda}_{n}-\frac{\epsilon}{\gamma}+\epsilon \gamma\right)+\sqrt{\Delta_{n}}}\right] \\
=\lim _{n \rightarrow \infty}\left[\frac{2\left(\tilde{\lambda}_{n}-\frac{\epsilon}{\gamma}+\epsilon \gamma\right) \epsilon \gamma}{\left(\tilde{\lambda}_{n}-\frac{\epsilon}{\gamma}+\epsilon \gamma\right)+\sqrt{\left(\tilde{\lambda}_{n}-\frac{\epsilon}{\gamma}+\epsilon \gamma\right)^{2}-4 \tilde{\lambda}_{n} \epsilon \gamma}}+\frac{2 \epsilon \gamma\left(\frac{\epsilon}{\gamma}-\epsilon \gamma\right)}{\left(\tilde{\lambda}_{n}-\frac{\epsilon}{\gamma}+\epsilon \gamma\right)+\sqrt{\left(\tilde{\lambda}_{n}-\frac{\epsilon}{\gamma}+\epsilon \gamma\right)^{2}-4 \tilde{\lambda}_{n} \epsilon \gamma}}\right]
\end{gathered}
$$




$$
=\lim _{n \rightarrow \infty}\left[\frac{2 \epsilon \gamma}{1+\sqrt{1-\frac{4 \tilde{\lambda}_{n} \epsilon \gamma}{\left(\tilde{\lambda}_{n}-\frac{\epsilon}{\gamma}+\epsilon \gamma\right)^{2}}}}+\frac{2 \epsilon \gamma\left(\frac{\epsilon}{\gamma}-\epsilon \gamma\right)}{\left(\tilde{\lambda}_{n}-\frac{\epsilon}{\gamma}+\epsilon \gamma\right)\left(1+\sqrt{1-\frac{4 \tilde{\lambda}_{n} \epsilon \gamma}{\left(\tilde{\lambda}_{n}-\frac{\epsilon}{\gamma}+\epsilon \gamma\right)^{2}}}\right)}\right],
$$

donde segue que

$$
\lim _{n \rightarrow \infty} \lambda_{n}^{-}=\epsilon \gamma .
$$

Portanto, a seqüência de autovalores $\left(\lambda_{n}^{-}\right)$do operador linear (2.41) converge para $\epsilon \gamma \in$ $\mathbb{R}$.

Um outro aspecto geométrico dos autovalores de (2.42) pode ser observado a partir da relação dada em (2.48). De fato, quando $\Delta_{n}<0$, sabemos que $\lambda_{n} \in \mathbb{C}$ e, da relação (2.48), considerando $\lambda_{n}=\alpha_{n}+i \beta_{n}$, com $\alpha_{n}, \beta_{n} \in \mathbb{R}$, temos

$$
\begin{gathered}
\left(\alpha_{n}+i \beta_{n}\right)+\frac{\epsilon^{2}}{\left(\alpha_{n}+i \beta_{n}\right)-\epsilon \gamma}+\frac{\epsilon}{\gamma} \in \mathbb{R} \\
\left(\alpha_{n}+\frac{\epsilon}{\gamma}+i \beta_{n}\right)+\frac{\epsilon^{2}\left[\left(\alpha_{n}-\epsilon \gamma\right)-i \beta_{n}\right]}{\left(\alpha_{n}-\epsilon \gamma\right)^{2}+\beta_{n}^{2}} \in \mathbb{R} \\
\left(\alpha_{n}+\frac{\epsilon}{\gamma}+\frac{\epsilon^{2}\left(\alpha_{n}-\epsilon \gamma\right)}{\left(\alpha_{n}-\epsilon \gamma\right)^{2}+\beta_{n}^{2}}\right)+i \beta_{n}\left(1-\frac{\epsilon^{2}}{\left(\alpha_{n}-\epsilon \gamma\right)^{2}+\beta_{n}^{2}}\right) \in \mathbb{R}
\end{gathered}
$$

donde segue que

$$
\left(\alpha_{n}-\epsilon \gamma\right)^{2}+\beta_{n}^{2}=\epsilon^{2}
$$

Portanto, quando $\Delta_{n}<0$, temos um número finito de autovalores $\lambda_{n} \in \mathbb{C}$ pertencentes a um círculo $\Gamma \subset \mathbb{C}$ de centro $(\epsilon \gamma, 0)$ e raio $\epsilon$.

Se tivermos $\Delta_{n} \geq 0$, então $\lambda_{n}^{ \pm} \in \mathbb{R}$ com $\lambda_{n}^{-} \leq \lambda_{n}^{+}$e com $\lambda_{n}^{-}$e $\lambda_{n}^{+}$conjugados em relação ao círculo $\Gamma$ uma vez que

$$
\left(\lambda_{n}^{-}-\epsilon \gamma\right)\left(\lambda_{n}^{+}-\epsilon \gamma\right)=\epsilon^{2}
$$

Esta é uma forma geométrica de ver que se $\lambda_{n}^{+} \longrightarrow+\infty$, então, correspondentemente, $\lambda_{n}^{-} \longrightarrow \epsilon \gamma$. Podemos reunir as observações acima na seguinte proposição

Proposição 2.4.1 Sejam $\left(\tilde{\lambda}_{n}\right)_{n \in \mathbb{N}}$ e, correspondentemente, $\left(\lambda_{n}^{ \pm}\right)_{n \in \mathbb{N}}$ as seqüências de autovalores associadas aos problemas (2.46) e (2.42), respectivamente. Então, (a). O número de autovalores complexos das seqüências $\left(\lambda_{n}^{ \pm}\right)_{n \in \mathbb{N}}$ é finito. Se existir algum autovalor complexo, então o seu conjugado também será um autovalor e ambos 
pertencerão ao círculo $\Gamma$ de centro $(\epsilon \gamma, 0)$ e raio $\epsilon$;

(b). A seqüência $\left(\lambda_{n}^{+}\right)_{n \in \mathbb{N}}$ diverge para $+\infty$ e a seqüência $\left(\lambda_{n}^{-}\right)_{n \in \mathbb{N}}$ converge para $\epsilon \gamma$;

(c). Os termos correspondentes das seqüências $\left(\lambda_{n}^{+}\right)_{n \in \mathbb{N}}$ e $\left(\lambda_{n}^{-}\right)_{n \in \mathbb{N}}$ são conjugados em relação ao círculo $\Gamma$.

Observamos que se, para algum $n \in \mathbb{N}$, tivermos $\Delta_{n}=0$, então, pela expressão (2.51), poderemos escrever $\left(\tilde{\lambda}_{n}-\epsilon \gamma-\frac{\epsilon}{\gamma}\right)^{2}=4 \epsilon^{2}$, donde $\tilde{\lambda}_{n}-\frac{\epsilon}{\gamma}+\epsilon \gamma=2 \epsilon(\gamma \pm 1)$. Da igualdade $\lambda_{n}=\frac{\tilde{\lambda}_{n}+\epsilon \gamma-\frac{\epsilon}{\gamma}}{2}$, vem $\lambda_{n}>0$, quando $\gamma>1$.

Como conseqüência das propriedades geométricas vistas acima temos a seguinte proposição.

Proposição 2.4.2 Se $u=\phi(x)$ for um equilibrio instável de (2.39), então, considerando $\psi(x)=\frac{1}{\gamma} \phi(x)$, o par $(\phi(x), \psi(x))$ será instável como equilíbrio de (2.36).

Demonstração. Consideremos $\mu_{0}$ o autovalor principal do problema (2.46). Por hipótese, $\mu_{0}<0$, donde $\Delta_{0}=\left(\mu_{0}-\frac{\epsilon}{\gamma}+\epsilon \gamma\right)^{2}-4 \mu_{0} \epsilon \gamma>0$, e, correspondentemente, $\lambda_{0}^{-}$e $\lambda_{0}^{+}$são reais. Além disso, como

$\sqrt{\Delta_{0}}=\sqrt{\left(\mu_{0}-\frac{\epsilon}{\gamma}+\epsilon \gamma\right)^{2}-4 \mu_{0} \epsilon \gamma}>\sqrt{\left(\mu_{0}-\frac{\epsilon}{\gamma}+\epsilon \gamma\right)^{2}}=\left|\mu_{0}-\frac{\epsilon}{\gamma}+\epsilon \gamma\right| \geq \mu_{0}-\frac{\epsilon}{\gamma}+\epsilon \gamma$ segue

$$
\lambda_{0}^{-}<0
$$

Proposição 2.4.3 Seja $\gamma>1$. Se $u=\phi(x)$ for um equilıbrio estável de (2.39), então o par $\left(\phi(x), \frac{1}{\gamma} \psi(x)\right)$ será estável como equilíbrio de (2.36).

Demonstração. Seja $\left(\mu_{n}\right)_{n \in \mathbb{N}}$ a seqüência de autovalores do problema (2.46). Por hipótese, $\mu_{n}>0$ para todo $n=0,1,2, \ldots$ e, de $\gamma>1$, vem $\gamma^{2}>\gamma>1$, donde $\gamma>\frac{1}{\gamma}$. Para qualquer $\epsilon>0$, vale $\mu_{n}+\epsilon \gamma-\frac{\epsilon}{\gamma}>0$. Se $\Delta_{n}<0$, então $\lambda_{n}^{ \pm} \in \mathbb{C}$ e $\operatorname{Re} \lambda_{n}^{ \pm}=\frac{\mu_{n}+\epsilon \gamma-\frac{\epsilon}{\gamma}}{2}>0$. Se $\Delta_{n} \geq 0$ os autovalores são reais com $\lambda_{n}^{-} \leq \lambda_{n}^{+}$. Da desigualdade

$$
\left(\mu_{n}+\epsilon \gamma-\frac{\epsilon}{\gamma}\right)^{2}>\left(\mu_{n}+\epsilon \gamma-\frac{\epsilon}{\gamma}\right)^{2}-4 \mu_{n} \epsilon \gamma=\Delta_{n} \geq 0
$$

vem $\left(\mu_{n}+\epsilon \gamma-\frac{\epsilon}{\gamma}\right)-\sqrt{\Delta_{n}}>0$ e $\lambda_{n}^{-}>0$, donde segue a estabilidade. 
Teorema 2.4.1 a) Se $\gamma>1$ e se existe $x_{0} \in(0,1)$ tal que $a^{\prime \prime}\left(x_{0}\right)>0$, então é possível determinar $f: \mathbb{R} \longrightarrow \mathbb{R}$, de classe $\mathscr{C}^{1}$, tal que, para todo $\epsilon>0$, o sistema (2.36) admite um equilíbrio não-constante $(\phi(x), \psi(x))$ estável.

b) Se $0<\gamma<1$ e se existe $x_{0} \in(0,1)$ tal que $a^{\prime \prime}\left(x_{0}\right)>0$, então é possível determinar $f: \mathbb{R} \longrightarrow \mathbb{R}$, de classe $\mathscr{C}^{1}$ e existe $\epsilon>0$ tais que o sistema (2.36) admite um equilíbrio não-constante $(\phi(x), \psi(x))$ estável.

Demonstração. Por um resultado bem conhecido de Yanagida [42] é possível encontrar uma função $\tilde{f}: \mathbb{R} \longrightarrow \mathbb{R}$, de classe $\mathscr{C}^{1}$, e uma função $u=\phi(x)$ tal que o problema

$$
\left\{\begin{array}{l}
u_{t}=\left(a^{2}(x) u_{x}\right)_{x}+\tilde{f}(u), 0<x<1, t>0 \\
u_{x}=0, x=0, x=1
\end{array}\right.
$$

admite $\phi$ como equilíbrio não-constante estável. Definindo $f(u)=\tilde{f}(u)+\frac{\epsilon}{\gamma} u$. temos que $\phi$ é um equilíbrio não constante estável de

$$
\left\{\begin{aligned}
u_{t}= & \left(a^{2}(x) u_{x}\right)_{x}+f(u)-\frac{\epsilon}{\gamma} u, \quad 0<x<1, t>0 \\
& u_{x}=0, \text { para } x=0, \quad x=1 .
\end{aligned}\right.
$$

Como $\gamma>1$, pela Proposição 2.4 .3 considerando $\psi=\frac{\epsilon}{\gamma} \phi$, temos que $(\phi, \psi)$ é equilíbrio não-constante estável do sistema

$$
\left\{\begin{aligned}
u_{t} & =\left(a^{2}(x) u_{x}\right)_{x}+f(u)-\epsilon v, \quad 0<x<1, t>0 \\
v_{t} & =\epsilon(u-\gamma v), \quad 0<x<1, t>0 \\
u_{x} & =0, \text { para } x=0, x=1
\end{aligned}\right.
$$

Sejam $\tilde{\lambda}_{1}$ e $\lambda_{1}$ os primeiros autovalores correspondentes aos problemas (2.54) e (2.55) respectivamente. De (2.49), vem que

$$
\lambda_{1}=\frac{\left(\tilde{\lambda}_{1}-\frac{\epsilon}{\gamma}+\epsilon \gamma\right) \pm \sqrt{\Delta_{1}}}{2}
$$

e de (2.51) vem que

$$
\Delta_{1}=\left(\tilde{\lambda}_{1}-\frac{\epsilon}{\gamma}+\epsilon \gamma\right)^{2}-4 \epsilon^{2}
$$

a) Se $\gamma>1$, então $0<\frac{1}{\gamma}<\gamma$, donde $0<-\frac{\epsilon}{\gamma}+\epsilon \gamma, \forall \epsilon>0$. Como $\tilde{\lambda}_{1}$ é positivo, concluimos que $0<\tilde{\lambda}_{1}<\tilde{\lambda}_{1}-\frac{\epsilon}{\gamma}+\epsilon \gamma, \forall \epsilon>0$. Temos três casos a considerar:

(i) Se $\Delta_{1}<0$, então $\lambda_{1} \in \mathbb{C}$ e, da conclusão acima, $\operatorname{Re}\left(\lambda_{1}\right)=\frac{\tilde{\lambda}_{1}-\frac{\epsilon}{\gamma}+\epsilon \gamma}{2}>0$. 
(ii) Se $\Delta=0$, então $\lambda_{1}=\frac{\dot{\lambda}_{1}-\frac{\epsilon}{\gamma}+\epsilon \gamma}{2}>0$.

(iii) Se $\Delta_{1}>0$, então $\lambda_{1}^{-}$e $\lambda_{1}^{+}$são reais com $\lambda_{1}^{-}<\lambda_{1}^{+}$, donde basta analisar o sinal de $\lambda_{1}^{-}$. De $\tilde{\lambda}_{1}>0$ vem que $\tilde{\lambda}_{1}-\frac{\epsilon}{\gamma}>-\frac{\epsilon}{\gamma}$ e $\gamma\left(\tilde{\lambda}_{1}-\frac{\epsilon}{\gamma}\right)>-\epsilon$, donde

$$
4 \epsilon \gamma\left(\tilde{\lambda}_{1}-\frac{\epsilon}{\gamma}\right)>-4 \epsilon^{2}, \quad \forall \gamma>0, \quad \forall \epsilon>0 .
$$

Desta última inequação segue

$$
\left(\tilde{\lambda}_{1}-\frac{\epsilon}{\gamma}\right)^{2}+2 \epsilon \gamma\left(\tilde{\lambda}_{1}-\frac{\epsilon}{\gamma}\right)+(\epsilon \gamma)^{2}>\left(\tilde{\lambda}_{1}-\frac{\epsilon}{\gamma}\right)^{2}-2 \epsilon \gamma\left(\tilde{\lambda}_{1}-\frac{\epsilon}{\gamma}\right)+(\epsilon \gamma)^{2}-4 \epsilon^{2},
$$

donde obtemos

$$
\left(\tilde{\lambda}_{1}-\frac{\epsilon}{\gamma}+\epsilon \gamma\right)^{2}>\left(\tilde{\lambda}_{1}-\frac{\epsilon}{\gamma}-\epsilon \gamma\right)^{2}-4 \epsilon^{2} .
$$

Como $\left(\tilde{\lambda}_{1}-\frac{\epsilon}{\gamma}+\epsilon \gamma\right)>0$ temos

$$
\lambda_{1}^{-}=\frac{\left(\tilde{\lambda}_{1}-\frac{\epsilon}{\gamma}+\epsilon \gamma\right)-\sqrt{\left(\tilde{\lambda}_{1}-\frac{\epsilon}{\gamma}-\epsilon \gamma\right)^{2}-4 \epsilon^{2}}}{2}>0 .
$$

Dos resultados de (i), (ii), (iii) segue que $(\phi, \psi)$ será estável como equilíbrio de (2.55).

b) Se $0<\gamma<1$, então $\frac{1}{\gamma}-\gamma>0$. Como $\tilde{\lambda}_{1}>0$ é possível escolher $\epsilon>0$ tal que $0<\epsilon\left(\frac{1}{\gamma}-\gamma\right)<\tilde{\lambda}_{1}$ de modo que se tenha $\tilde{\lambda}_{1}-\frac{\epsilon}{\gamma}+\epsilon \gamma>0$. Podemos obter conclusões análogas às obtidas em (i), (ii) e (iii), donde segue a estabilidade do equilíbrio $(\phi, \psi)$.

Teorema 2.4.2 Se $a^{\prime \prime}\left(x_{0}\right)>0$ para algum $x_{0} \in(0,1)$ então, para cada $\gamma>0$ dado, existe $f: \mathbb{R} \longrightarrow \mathbb{R}$, de classe $\mathscr{C}^{1}$, e existe $\epsilon>0$ tais que o sistema (2.36) tenha um equilibrio não-constante estável.

Demonstração. Segundo Yanagida [42] é possível definir $\phi=\phi(x)$ e $\tilde{f}$, de classe $\mathscr{C}^{1}$, tal que

$$
\left\{\begin{array}{l}
u_{t}=\left(a^{2}(x) u_{x}\right)_{x}+\tilde{f}(u), \quad 0<x<1, t>0 \\
u_{x}=0, \quad x=0, \quad x=1 .
\end{array}\right.
$$

admita $\phi$ como equilíbrio não constante estável. Definindo $f(u)=\tilde{f}(u)+\frac{\epsilon}{\gamma} u$ temos que $f$ é de classe $\mathscr{C}^{1}$.

Considerando, então, $\psi=\frac{\epsilon}{\gamma} \phi$, temos que $(\phi, \psi)$ é equilíbrio não constante do sistema

$$
\left\{\begin{aligned}
u_{t} & =\left(a^{2}(x) u_{x}\right)_{x}+f(u)-\epsilon v, \quad 0<x<1, t>0 \\
v_{t} & =\epsilon(u-\gamma v), \quad 0<x<1, t>0 \\
u_{x} & =0, \text { para } x=0, x=1 .
\end{aligned}\right.
$$


Fixando $v=\psi=\frac{1}{\gamma} \phi$ na primeira equação de (2.57) temos

$$
\left\{\begin{array}{l}
u_{t}=\left(a^{2}(x) u_{x}\right)_{x}+f(u)-\frac{\epsilon}{\gamma} \phi(x), \quad 0<x<1, \quad t>0 \\
u_{x}=0, \quad \text { para } x=0,1 .
\end{array}\right.
$$

Temos que $u=\phi$ é equilíbrio do sistema (2.58). O problema de autovalor correspondente a (2.58) é expresso por:

$$
\left\{\begin{aligned}
\left(a^{2}(x) u^{\prime}\right)^{\prime}+f^{\prime}(\phi) u+\bar{\lambda} u & =0, \quad 0<x<1 \\
u^{\prime}(0)=u^{\prime}(1) & =0 .
\end{aligned}\right.
$$

Por outro lado, como $u=\phi$ é equilíbrio estável de (2.56), então $\tilde{\lambda}_{1}>0$ onde $\tilde{\lambda}_{1}$ é o primeiro autovalor para o problema de autovalor associado ao problema (2.56), dado por:

$$
\left\{\begin{aligned}
\left(a^{2}(x) u^{\prime}\right)^{\prime}+\tilde{f}^{\prime}(\phi) u+\tilde{\lambda} & =0, \quad 0<x<1 \\
u^{\prime}(0)=u^{\prime}(1) & =0 .
\end{aligned}\right.
$$

Notando que $f^{\prime}(\phi)=\tilde{f}^{\prime}(\phi)+\frac{\epsilon}{\gamma}$, da comparação entre os problemas (2.59) e (2.60) temos que, para cada $n \in \mathbb{N}$, vale a relação

$$
\tilde{\lambda}_{n}=\bar{\lambda}_{n}+\frac{\epsilon}{\gamma}
$$

a) Se $\gamma>1$, então $0<\frac{1}{\gamma}<1$ e $-\frac{\epsilon}{\gamma}>-\epsilon, \forall \epsilon>0$, donde

$$
\bar{\lambda}_{1}=\tilde{\lambda}_{1}-\frac{\epsilon}{\gamma}>\tilde{\lambda}_{1}-\epsilon
$$

Como $\tilde{\lambda}_{1}>0$, podemos escolher $\epsilon>0$ tal que $0<\epsilon<\tilde{\lambda}_{1}$ o que implica $\bar{\lambda}_{1}>0$.

b) Se $0<\gamma<1$, então $\frac{1}{\gamma}>1$ e podemos escolher $\epsilon>0$ tal que $0<\frac{\epsilon}{\gamma}<\tilde{\lambda}_{1}$ o que implica $\bar{\lambda}_{1}>0$. Portanto, em qualquer um dos casos $a$ ), $b$ ) considerados acima é possível escolher $\epsilon>0$ tal que $\bar{\lambda}_{1}>0$. Donde segue que $u=\phi$ é estável como equilíbrio de (2.58).

Fixando, agora, $u=\phi$ na segunda equação de (2.57) temos

$$
\begin{gathered}
v_{t}=\epsilon \phi(x)-\epsilon \gamma v, \quad 0<x<1, \quad t>0 . \\
\frac{\partial}{\partial t}\left(e^{\epsilon \gamma t} v(x, t)\right)=\epsilon \phi(x) e^{\epsilon \gamma t}, \\
v(x, t) e^{\epsilon \gamma t}-v(x, 0)=\frac{1}{\gamma} \phi(x)\left(e^{\epsilon \gamma t}-1\right),
\end{gathered}
$$




$$
\begin{gathered}
v(x, t)=\frac{1}{\gamma} \phi(x)+\left(v(x, 0)-\frac{1}{\gamma} \phi(x)\right) e^{-\epsilon \gamma t}, \\
v(x, t)=\psi(x)+\left(v_{0}(x)-\psi(x)\right) e^{-\epsilon \gamma t} .
\end{gathered}
$$

Portanto, para quaisquer $\gamma>0, \epsilon>0$, temos que $v=\psi$ é equilíbrio exponencialmente estável de (2.61). Pelo Teorema (2.3.1) temos que $(\phi(x), \psi(x))$ é equilíbrio não constante estável de (2.36).

Teorema 2.4.3 Se $a^{\prime \prime}(x) \leq 0, \forall x \in(0,1)$, então todo equilibrio não-constante $(\phi, \psi)$ de (2.36) será instável.

Demonstração. Seja $(\phi, \psi)$ equilíbrio não-constante de (2.36). Então, pela equação (2.42) temos que $\psi=\frac{1}{\gamma} \phi$, de modo que $u=\phi$ é equilíbrio não-constante de (2.39). Por Yanagida [42] temos que $\phi$ é instável como equilíbrio de (2.39), donde $\tilde{\lambda}_{1}$, o primeiro autovalor do problema (2.46) é negativo. Para obter o resultado basta considerar o autovalor $\lambda_{1}^{-}$.

De fato, de (2.50) temos

$$
\Delta_{1}=\left(\tilde{\lambda}_{1}-\frac{\epsilon}{\gamma}+\epsilon \gamma\right)^{2}-4 \tilde{\lambda}_{1} \epsilon \gamma
$$

e, de $\tilde{\lambda}_{1}<0$, concluimos que $\Delta_{1}>0$, donde $\lambda_{1}$ é real e vale $\lambda_{1}^{-}<\lambda_{1}^{+}$.

Pode ocorrer $\left(\tilde{\lambda}_{1}-\frac{\epsilon}{\gamma}+\epsilon \gamma\right)<0$ ou $\left(\tilde{\lambda}_{1}-\frac{\epsilon}{\gamma}+\epsilon \gamma\right) \geq 0$. No primeiro caso, a condição $\left(\tilde{\lambda}_{1}-\frac{\epsilon}{\gamma}+\epsilon \gamma\right)<0$ implica

$$
\lambda_{1}^{-}<0 \text {. }
$$

Por outro lado, se $\left(\tilde{\lambda}_{1}-\frac{\epsilon}{\gamma}+\epsilon \gamma\right) \geq 0$, da condição $\tilde{\lambda}_{1}<0$ e da expressão (2.50) vem

$$
\left(\tilde{\lambda}_{1}-\frac{\epsilon}{\gamma}+\epsilon \gamma\right)^{2}<\left(\tilde{\lambda}_{1}-\frac{\epsilon}{\gamma}+\epsilon \gamma\right)^{2}-4 \tilde{\lambda}_{1} \epsilon \gamma=\Delta_{1}
$$

e

$$
\left(\tilde{\lambda}_{1}-\frac{\epsilon}{\gamma}+\epsilon \gamma\right)-\sqrt{\Delta_{1}}<0
$$

donde $\lambda_{1}^{-}<0$.

Portanto, em qualquer um dos casos temos $\lambda_{1}^{-}<0$, donde $(\phi, \psi)$ é instável como equilíbrio de (2.36). 
Observação 2.4.1 Se o coeficiente $a$ do sistema (2.36) for constante, então todo equilíbrio não constante será instável.

Observação 2.4.2 Consideremos $\mathscr{E}_{1}$ o conjunto dos equilíbrios do sistema (2.36) e $\mathscr{E}_{2}$ o conjunto dos equilíbrios da equação (2.39). Então,

$$
\mathscr{E}_{1} \equiv \mathscr{E}_{2}
$$

Observação 2.4.3 (1.1) $\phi \in \mathscr{E}_{2}$ estável, $\gamma>1 \Longrightarrow\left(\phi, \frac{1}{\gamma} \phi\right) \in \mathscr{E}_{1}$ estável.

(1.2) $\left(\phi, \frac{1}{\gamma} \phi\right) \in \mathscr{E}_{1}$ estável, $\gamma>1 \Longrightarrow \phi \in \mathscr{E}_{2}$ estável. 


\section{Capítulo 3}

\section{Sistemas Anti-Gradientes}

Neste capítulo vamos abordar o problema da instabilidade de equilíbrios não-constantes para sistemas de equações diferenciais parciais do tipo parabólico, equações de reaçãodifusão, com estrutura anti-gradiente definidas num domínio limitado $\Omega$ do $\mathbb{R}^{N}$, com condições de Neumann homogêneas na fronteira. Sistemas de equações difenciais parciais com coeficientes constantes já têm sido motivo de extensos estudos desde algum tempo, mas os casos com coeficientes variáveis só mais recentemente têm sido tratados ver, por exemplo, em Willie [41].

Vimos no Capítulo 1 algumas condições em que o sistema constituído por uma equação possui um equilíbrio não-constante instável. Para os sistemas com mais de uma equação escalar, as condições geométricas de convexidade do domínio não são suficientes para garantir a instabilidade de equilíbrios não-constantes como se viu no Exemplo 2.3.2. Em dimensão $N \geq 2$, para as equações com coeficientes constantes, a convexidade do domínio determina a instabilidade dos equilíbrios não-constantes conforme Casten e Holland [7] e Matano [33]. No caso de sistemas de equações com coeficientes constantes também há uma certa influência da geometria do domínio na estabilidade dos equilíbrios conforme Yanagida [43] e Lopes [31]. Veremos que propriedades análogas também permanecem válidas no caso de sistemas de equações com coeficientes variáveis definidas em alguns domínios convexos particulares. 


\subsection{Formulação Geral}

Seja o sistema de equações de reação-difusão dado por

$$
\left\{\begin{aligned}
R u_{t} & =\operatorname{div}(A(x) \nabla u)+f(u, v), \quad x \in \Omega, \quad t>0 \\
S v_{t} & =\operatorname{div}(B(x) \nabla v)+g(u, v), \quad x \in \Omega, \quad t>0 \\
\frac{\partial u}{\partial \nu} & =\frac{\partial v}{\partial \nu}=0, \quad x \in \partial \Omega, t>0,
\end{aligned}\right.
$$

onde $\Omega \subset \mathbb{R}^{N}$ é um domínio limitado com fronteira $\partial \Omega$ de classe $\mathscr{C}^{k}, k \geq 2, \vec{n}$ é o vetor normal exterior em $\partial \Omega, R: \Omega \longrightarrow \mathbb{M}_{m}(\mathbb{R})$, matriz diagonal positiva de ordem $m, A: \Omega \longrightarrow\left(\mathbb{M}_{N}(\mathbb{R})\right)^{m}$ é uma função de classe $\mathscr{C}^{2}$ que a cada $x \in \Omega$ associa uma $m-$ upla de matrizes diagonais positivas $A_{i}(x)=\operatorname{diag}\left\{a_{i 1}^{2}(x), \ldots, a_{i N}^{2}(x)\right\}$ de ordem $N$, $S: \Omega \longrightarrow \mathbb{M}_{n}(\mathbb{R})$ matriz diagonal positiva de ordem $n$ e $B: \Omega \longrightarrow\left(\mathbb{M}_{N}(\mathbb{R})\right)^{n}$ a função de classe $\mathscr{C}^{2}$ que a cada $x \in \Omega$ faz corresponder a uma $n$ - upla de matrizes diagonais positivas $B_{k}(x)=\operatorname{diag}\left\{b_{k 1}^{2}(x) \ldots, b_{k N}^{2}(x)\right\}$ de ordem $N$. As derivadas co-normais de $u$ e de $v$ satisfazem $\frac{\partial u}{\partial \nu}=\frac{\partial v}{\partial \nu}=0$ em $\partial \Omega$ e $f: \mathbb{R}^{m+n} \longrightarrow \mathbb{R}^{m}$ e $g: \mathbb{R}^{m+n} \longrightarrow \mathbb{R}^{n}$ são funções de classe $\mathscr{C}^{1}$.

NOTAÇÃO. Fixemos as notações seguintes: $u: \Omega \rightarrow \mathbb{R}^{m}, u(x)=\left(u_{1}(x), \ldots, u_{m}(x)\right)^{T}$, com $u_{i}: \Omega \rightarrow \mathbb{R}, 1 \leq i \leq m$, com análogo para a função $v: \Omega \rightarrow \mathbb{R}^{n}, v(x)=$ $\left(v_{1}(x), \ldots, v_{n}(x)\right)^{T}$. Indiquemos com a notação gradiente $\nabla u=\left(\nabla u_{1}, \ldots, \nabla u_{m}\right)$, onde $\nabla u_{i}=\left(\frac{\partial u_{i}}{\partial x_{1}}, \ldots, \frac{\partial u_{i}}{\partial x_{N}}\right)^{T}, 1 \leq i \leq m$, com análogo para $\nabla v=\left(\nabla v_{1}, \ldots, \nabla v_{n}\right)$. Para $A(x)=\left(A_{1}(x), \ldots, A_{m}(x)\right)$ denotamos

$$
\operatorname{div}(A(x) \nabla u)=\left(\operatorname{div}\left(A_{1} \nabla u_{1}\right), \ldots, \operatorname{div}\left(A_{m} \nabla u_{m}\right)\right)^{T},
$$

com expressão análoga para $\operatorname{div}(B(x) \nabla v)$. Indicamos $\frac{\partial u}{\partial \nu}=\langle A \nabla u, \vec{n}\rangle=\left(\frac{\partial u_{1}}{\partial \nu}, \ldots, \frac{\partial u_{m}}{\partial \nu}\right)^{T}$ a derivada co-normal, onde $\frac{\partial u_{i}}{\partial \nu}=\left\langle A_{i} \nabla u_{i}, \vec{n}\right\rangle, 1 \leq i \leq m$, indica a derivada conormal que vimos usando anteriormente. Usamos expressão análoga para $\frac{\partial v}{\partial \nu}$. Denotamos $\langle A \nabla u, \nabla v\rangle=\sum_{i=1}^{m}\left(A_{i} \nabla u_{i}\right) \cdot \nabla v_{i}=\sum_{i=1}^{m}\left(\sum_{k=1}^{N} a_{i k}^{2} \frac{\partial u_{i}}{\partial x_{k}} \frac{\partial v_{i}}{\partial x_{k}}\right)$.

Lembramos que, com a estrutura anti-gradiente, conforme a Definição 2.1.1, o sistema (3.1) se escreve como

$$
(P N)=\left\{\begin{aligned}
R u_{t} & =\operatorname{div}(A(x) \nabla u)+H_{u}(u, v), \quad x \in \Omega, t>0 \\
S v_{t} & =\operatorname{div}(B(x) \nabla v)-H_{v}(u, v), \quad x \in \Omega, t>0 \\
\frac{\partial u}{\partial \nu} & =\frac{\partial v}{\partial \nu}=0 \text { em } \Omega, t>0,
\end{aligned}\right.
$$

onde fica evidente o acoplamento anti-simétrico de dois sistemas do tipo gradiente. 
Definição 3.1.1 Dizemos que o par de funções $(u, v)=(\phi, \psi): \Omega \longrightarrow \mathbb{R}^{2} \phi, \psi \in$ $\mathscr{C}^{1}(\bar{\Omega}) \cap \mathscr{C}^{2}(\Omega)$ é um equilíbrio do problema (3.1) se a condição seguinte estiver verificada:

$$
\left\{\begin{aligned}
\operatorname{div}(A(x) \nabla \phi)+f(\phi, \psi) & =0, \quad x \in \Omega, \\
\operatorname{div}(B(x) \nabla \psi)+g(\phi, \psi) & =0, \quad x \in \Omega, \\
\frac{\partial \phi}{\partial \nu} & =\frac{\partial \psi}{\partial \nu}=0 \operatorname{em} \Omega .
\end{aligned}\right.
$$

Se $(u, v)=(\phi(x), \psi(x))$ for um equilíbrio do problema (3.1), então indicamos a linearização referente a $(\phi, \psi)$ que será dada pelo seguinte sistema linear:

$$
\left\{\begin{aligned}
R U_{t} & =(A(x) \nabla U)+f_{u}(\phi, \psi) U+f_{v}(\phi, \psi) V, x \in \Omega, t>0 \\
S V_{t} & =(B(x) \nabla V)+g_{u}(\phi, \psi) U+g_{v}(\phi, \psi) V, x \in \Omega, t>0 \\
\frac{\partial U}{\partial \nu} & =\frac{\partial V}{\partial \nu}=0 \text { em } \Omega
\end{aligned}\right.
$$

Consideremos o seguinte espaço funcional $X=\left(\mathbf{L}^{2}(\Omega)\right)^{m} \times\left(\mathbf{L}^{2}(\Omega)\right)^{n}$ munido da norma proveniente do produto interno definido por

$$
\langle u, v\rangle=\int_{\Omega}\left(\sum_{j=1}^{m} u_{j} \bar{u}_{j}+\sum_{k=1}^{n} v_{k} \bar{v}_{k}\right) \mathrm{d} x=\int_{\Omega}(u(x) \cdot \bar{u}(x)+v(x) \cdot \bar{v}(x)) \mathrm{d} x
$$

para todo $u \in\left(\mathbf{L}^{2}(\Omega)\right)^{m}$ e todo $v \in\left(\mathbf{L}^{2}(\Omega)\right)^{m}$, onde a barra significa a conjugação complexa. Sejam os operadores lineares $\mathbb{A}: D(\mathbb{A}) \subset X \rightarrow X$ e $\mathbb{B}: D(\mathbb{B}) \longrightarrow X$, associados ao problema (3.1), dados, respectivamente, por $\mathbb{A} u=-\operatorname{div}(A(x) \nabla u)$ e $\mathbb{B} v=$ $-\operatorname{div}(B(x) \nabla v)$ onde $D(\mathbb{A})=\left\{u \in\left(\mathbf{H}^{2}(\Omega)\right)^{m} ; \frac{\partial u}{\partial \nu}=0 ;\right.$ em $\left.\partial \Omega\right\}$ e $D(\mathbb{B})=\{v \in$ $\left(\mathbf{H}^{2}(\Omega)\right)^{n} ; \frac{\partial v}{\partial \nu}=0 ;$ em $\left.\partial \Omega\right\}$.

Associado ao sistema linearizado (3.3) consideremos o operador linear $\mathscr{L}:\left(\mathbf{H}^{2}(\Omega)\right)^{m} \times$ $\left(\mathbf{H}^{2}(\Omega)\right)^{n} \longrightarrow\left(\mathbf{L}^{2}(\Omega)\right)^{m+n}$ definido por

$$
\mathscr{L}\left(\begin{array}{c}
U \\
V
\end{array}\right)=-\left(\begin{array}{c}
\operatorname{div}(A(x) \nabla U) \\
\operatorname{div}(B(x) \nabla V)
\end{array}\right)-\left(\begin{array}{cc}
f_{u}(\phi, \psi) & f_{v}(\phi, \psi) \\
g_{u}(\phi, \psi) & g_{v}(\phi, \psi)
\end{array}\right)\left(\begin{array}{c}
U \\
V
\end{array}\right),
$$

para todo $(u, v) \in\left(\mathbf{H}^{2}(\Omega)\right)^{m} \times\left(\mathbf{H}^{2}(\Omega)\right)^{n}$ satisfazendo as condições de fronteira $\frac{\partial U}{\partial \nu}=$ $\frac{\partial V}{\partial \nu}=0$ em $\partial \Omega$, onde $f_{u}, f_{v}, g_{u}$ e $g_{v}$ são matrizes tais que $f_{u}(\phi, \psi) \in \mathbb{M}_{m \times m}(\mathbb{R}), f_{v}(\phi, \psi) \in$ $\mathbb{M}_{m \times n}(\mathbb{R}), g_{u}(\phi, \psi) \in \mathbb{M}_{n \times m}(\mathbb{R}), g_{v}(\phi, \psi) \in \mathbb{M}_{n \times n}(\mathbb{R})$ e, pela estrutura anti-gradiente do sistema (3.1), $f_{v}(\phi, \psi)^{T}=-g_{u}(\phi, \psi)$.

O estudo da estabilidade dos equilíbrios do sistema (3.1) reduz-se ao estudo do problema de auto-valor para o operador $\mathscr{L}$ associado, problema linearizado (3.3), com 
condição de Neumann homogênea na fronteira, expresso por

$$
(P A)=\left\{\begin{array}{l}
\mathscr{L}[w]=\lambda T w, \quad x \in \Omega \\
\frac{\partial w}{\partial \nu}(x)=0, \quad x \in \partial \Omega
\end{array}\right.
$$

onde, $T=(R, S), w=(u, v)$ e $T w=(R u, S v)$.

Como o operador linear $\mathscr{L}$ associado ao equilíbrio $(\phi, \psi)$ do sistema anti-gradiente (3.1), definido em (3.4), não é auto-adjunto, seus autovalores podem ser complexos. Entretanto, justamente pela estrutura anti-gradiente de (3.1), podemos dividir o problema da análise da instabilidade do equilíbrio do sistema dado em dois sistemas parciais correspondentes. Analogamente ao que foi feito no caso unidimensional, vamos explorar determinadas propriedades de anti-simetria de (3.1). Para tanto, consideremos o seguinte problema

$$
\left\{\begin{aligned}
-\operatorname{div}(A(x) \nabla U)-f_{u}(\phi, \psi) U-f_{v}(\phi, \psi) V & =\lambda R U, \quad x \in \Omega, \\
-\operatorname{div}(B(x) \nabla V)-g_{u}(\phi, \psi) U-g_{v}(\phi, \psi) V & =\lambda S V, \quad x \in \Omega, \\
\frac{\partial U}{\partial \nu}=\frac{\partial V}{\partial \nu} & =0, \quad x \in \partial \Omega,
\end{aligned}\right.
$$

e vamos procurar $\lambda \in \mathbb{C}$ para o qual o sistema admite solução não-nula.

Observamos que, se $(U, V)$ é uma solução do sistema (3.5), então, usando o Teorema da Divergência, com as condições de fronteira temos as igualdades seguintes

$$
\int_{\Omega}-\bar{U} \cdot \operatorname{div}(A(x) \nabla U) \mathrm{d} x=\int_{\Omega}\langle A(x) \nabla U, \nabla \bar{U}\rangle \mathrm{d} x
$$

e

$$
\int_{\Omega}-V \cdot \operatorname{div}(B(x) \nabla \bar{V}) \mathrm{d} x=\int_{\Omega}\langle B(x) \nabla \bar{V}, \nabla V\rangle \mathrm{d} x .
$$

Pela estrutura anti-gradiente do sistema (3.1) tem-se

$$
f_{v}(\phi, \psi) V \cdot \bar{U}+g_{u}(\phi, \psi) \bar{U} \cdot V=0 .
$$

Portanto, integrando sobre o domínio $\Omega$, a soma da primeira equação de (3.5) multiplicada escalarmente pelo vetor $\bar{U}$ com a conjugada da segunda equação multiplicada escalarmente pelo vetor $V$ obtemos

$$
\begin{aligned}
\lambda \int_{\Omega} R U \cdot \bar{U} \mathrm{~d} x+\bar{\lambda} \int_{\Omega} S \bar{V} \cdot V \mathrm{~d} x=\int_{\Omega}\left(\langle A \nabla U, \nabla \bar{U}\rangle-f_{u}(\phi, \psi) U \cdot \bar{U}\right) \mathrm{d} x & \\
& +\int_{\phi_{\Omega}}\left(\langle B \nabla \bar{V}, \nabla V\rangle-g_{v}(\phi, \psi) \bar{V} \cdot V\right) \mathrm{d} x .
\end{aligned}
$$


Definindo os funcionais $I:\left(\mathbf{H}^{1}(0,1)\right)^{m} \longrightarrow \mathbb{R}$ e $J:\left(\mathbf{H}^{1}(0,1)\right)^{n} \longrightarrow \mathbb{R}$ por

$$
I[U]=\int_{\Omega}\left(\langle A \nabla U, \nabla \bar{U}\rangle-f_{u}(\phi, \psi) U \cdot \bar{U}\right) \mathrm{d} x, \quad \forall U \in\left(\mathbf{H}^{1}(0,1)\right)^{m},
$$

e

$$
J[V]=\int_{\Omega}\left(\langle B \nabla \bar{V}, \nabla V\rangle-g_{v}(\phi, \psi) \bar{V} \cdot V\right) \mathrm{d} x, \quad \forall V \in\left(\mathbf{H}^{1}(0,1)\right)^{n},
$$

podemos escrever (3.6) como

$$
\lambda \int_{\Omega} R U \cdot \bar{U} \mathrm{~d} x+\bar{\lambda} \int_{\Omega} S \bar{V} \cdot V \mathrm{~d} x=I[U]+J[V] .
$$

Se o autovalor $\lambda$ do operador (3.4) for dado como $\lambda=\alpha+i \beta$ onde $\alpha$ e $\beta$ são reais, então poderemos escrever

$$
\begin{aligned}
\lambda \int_{\Omega} R U \cdot \bar{U} \mathrm{~d} x+\bar{\lambda} \int_{\Omega} S \bar{V} \cdot V \mathrm{~d} x= & \alpha\left(\int_{\Omega} R U \cdot \bar{U} \mathrm{~d} x+\int_{\Omega} S \bar{V} \cdot V \mathrm{~d} x\right) \\
& +i \beta\left(\int_{\Omega} R U \cdot \bar{U} \mathrm{~d} x-\int_{\Omega} S \bar{V} \cdot V \mathrm{~d} x\right) .
\end{aligned}
$$

Da expressão acima e da igualdade (3.6) vem

$$
\operatorname{Re}(\lambda)\left(\int_{\Omega} \mathrm{RU} \cdot \overline{\mathrm{U}} \mathrm{dx}+\int_{\Omega} \mathrm{S} \overline{\mathrm{V}} \cdot \mathrm{Vdx}\right)=\mathrm{I}[\mathrm{U}]+\mathrm{J}[\mathrm{V}]
$$

Analogamente ao caso dos sistemas unidimensionais, a expressão (3.7) mostra que, o estudo da estabilidade linear dos equilíbrios do sistema anti-gradiente (3.1) resume-se ao estudo dos sinais dos funcionais $I, J$.

\subsection{Sistemas Parciais}

Consideremos o sistema (3.1) no caso particular $m=n=1$ uma vez que para $m>1$ e $n>1$ o estudo é análogo, com as adaptações correspondentes. Neste caso, $f, g$ : $\mathbb{R}^{2} \longrightarrow \mathbb{R}$ são de classe $\mathscr{C}^{1}$ tais que existe uma função $H: \mathbb{R}^{2} \longrightarrow \mathbb{R}$ satisfazendo $f(u, v)=h_{u}(u, v)$ e $g(u, v)=-h_{v}(u, v)$, de modo que a condição de anti-gradiente do sistema (3.1) dado corresponde a $f_{v}+g_{u}=0$ em $\mathbb{R}^{2}$.

Tendo em vista a relação (3.7), vamos analisar independentemente os sistemas parciais que compõem o sistema (3.1). Considerando $(\phi, \psi)$ um equilíbrio do sistema dado fixemos, inicialmente, $v=\psi(x)$ na primeira equação, obtemos o seguinte sistema parcial

$$
\left\{\begin{aligned}
R u_{t} & =\operatorname{div}(A(x) \nabla u)+f(u, \psi(x)), \quad x \in \Omega, \quad t>0 \\
\frac{\partial u}{\partial \nu} & =0, \quad x \in \partial \Omega, \quad t>0
\end{aligned}\right.
$$


É imediato verificar que $\phi$ é equilíbrio deste sistema.

Indiquemos o linearizado do sistema parcial (3.8) em torno do equilíbrio $\phi$ por

$$
\left\{\begin{aligned}
R U_{t} & =\operatorname{div}(A(x) \nabla U)+f_{u}(\phi, \psi(x)) U, \quad x \in \Omega, \quad t>0 \\
\frac{\partial U}{\partial \nu} & =0, \quad x \in \partial \Omega, \quad t>0
\end{aligned}\right.
$$

e o operador linear $\mathscr{L}_{1}$ definido em $\left(\mathbf{H}^{2}(\Omega)\right)$ dado por

$$
\mathscr{L}_{1}[u]=-\operatorname{div}(A(x) \nabla u)-f_{u}(\phi, \psi) u, \quad \frac{\partial u}{\partial \nu}=0 \quad \text { em } \quad \partial \Omega .
$$

Observando que o operador $\mathscr{L}_{1}$ é auto-adjunto, para análise dos sinais dos seus autovalores, consideremos o sistema

$$
\left\{\begin{aligned}
\mathscr{L}_{1} U & =\mu R U, \quad x \in \Omega \\
\frac{\partial U}{\partial \vec{n}} & =0, \quad x \in \partial \Omega
\end{aligned}\right.
$$

Para tanto, consideremos o funcional quadrático $I:\left(\mathbf{H}^{1}(\Omega)\right) \longrightarrow \mathbb{R}$ dado por

$$
I[u]=\int_{\Omega}\left[\langle A \nabla u, \nabla u\rangle-f_{u}(\phi, \psi) u^{2}\right] \mathrm{d} x, \forall u \in \mathbf{H}^{1}(\Omega) .
$$

Se existir $\xi \in\left(\mathbf{H}^{1}(\Omega)\right)$ tal que $I(\xi) \leq 0$, então o auto-valor principal $\mu_{1}$ do operador $\mathscr{L}_{1}$ é negativo e, como conseqüência, $\phi$ é instável como equilíbrio de (3.8).

Para determinar o sinal de $I$ vamos escolher funções adequadas. Se $\phi$ é equilíbrio não-constante então, podemos calcular o funcional nas funções $u=a_{j} \phi_{x_{j}}, 1 \leq j \leq N$,

$$
I\left[a_{j} \phi_{x_{j}}\right]=\int_{\Omega}\left[\left\langle A(x) \nabla\left(a_{j} \phi_{x_{j}}\right), \nabla\left(a_{j} \phi_{x_{j}}\right)\right\rangle-f_{u}(\phi, \psi)\left(a_{j} \phi_{x_{j}}\right)^{2}\right] \mathrm{d} x, x \in \Omega .
$$

Sem perder a generalidade, vamos considerar o sistema (3.8) definido num domínio limitado $\Omega$ do plano $\mathbb{R}^{2}$ uma vez que o caso geral decorre facilmente deste. As matrizes das difusividades serão dadas por $A(x, y)=\operatorname{diag}\left\{a_{1}^{2}, a_{2}^{2}\right\}$ e $B(x, y)=\operatorname{diag}\left\{b_{1}^{2}, b_{2}^{2}\right\}$. Indicaremos o sistema (3.8), particularmente, por

$$
\left\{\begin{aligned}
R u_{t} & =\left(a_{1}^{2}(x, y) u_{x}\right)_{x}+\left(a_{2}^{2}(x, y) u_{y}\right)_{y}+f(u, \psi), \quad(x, y) \in \Omega, \quad t>0 \\
\frac{\partial u}{\partial \nu} & =a_{1}^{2} u_{x} n_{1}+a_{2}^{2} u_{y} n_{2}=0, \quad(x, y) \in \partial \Omega, \quad t>0,
\end{aligned}\right.
$$

Nessas condições, temos

$$
I\left[a_{1} \phi_{x}\right]=\int_{\Omega}\left\langle A(x, y) \nabla\left(a_{1} \phi_{x}\right), \nabla\left(a_{1} \phi_{x}\right)\right\rangle-f_{u}(\phi, \psi)\left(a_{1} \phi_{x}\right)^{2}
$$


e

$$
I\left[a_{2} \phi_{y}\right]=\int_{\Omega}\left\langle A(x, y) \nabla\left(a_{2} \phi_{y}\right), \nabla\left(a_{2} \phi_{y}\right)\right\rangle-f_{u}(\phi, \psi)\left(a_{2} \phi_{y}\right)^{2} .
$$

Pela propriedade do divergente temos

$$
I\left[a_{1} \phi_{x}\right]=\frac{1}{2} \int_{\partial \Omega} \frac{\partial}{\partial \nu}\left(a_{1} \phi_{x}\right)^{2}+\int_{\Omega}-\left(a_{1} \phi_{x}\right)\left(\operatorname{div}\left(A \nabla\left(a_{1} \phi_{x}\right)\right)+f_{u}(\phi, \psi)\left(a_{1} \phi_{x}\right)\right) \mathrm{d} x .
$$

Indicando por $\Sigma_{x}$ o integrando da segunda integral em (3.12), da condição de equilíbrio do sistema dado, $\operatorname{div}(A(x, y) \nabla \phi)+f(\phi, \psi)=0$, e das propriedades do divergente, podemos escrever:

$$
\Sigma_{x}=a_{1}^{2} \phi_{x} \operatorname{div}\left(A_{x} \nabla \phi\right)-a_{1} \phi_{x}\left\langle A \nabla \phi_{x}, \nabla a_{1}\right\rangle-a_{1} \phi_{x} \operatorname{div}\left(\phi_{x} A \nabla a_{1}\right)+f_{v}(\phi, \psi) a_{1}^{2} \phi_{x} \psi_{x} .
$$

A expressão $\Sigma_{x}$ pode ser re-escrita como

$$
\begin{aligned}
\Sigma_{x}=\operatorname{div}\left(a_{1}^{2} \phi_{x} A_{x} \nabla \phi\right)-\frac{1}{2} \operatorname{div}\left(\phi_{x}^{2} A \nabla a_{1}^{2}\right) & -\left\langle\nabla \phi, A_{x} \nabla\left(a_{1}^{2} \phi_{x}\right)\right\rangle \\
& +\left\langle A \nabla a_{1}, \nabla a_{1}\right\rangle \phi_{x}^{2}+f_{v}(\phi, \psi) a_{1}^{2} \phi_{x} \psi_{x} .
\end{aligned}
$$

Assim, a segunda integral da expressão (3.12) se escreve como

$$
\begin{aligned}
\int_{\Omega} \Sigma_{x}=\int_{\partial \Omega}\left[\left\langle a_{1}^{2} \phi_{x} A_{x} \nabla \phi, \vec{n}\right\rangle-\frac{1}{2}\left\langle\phi_{x}^{2} A \nabla a_{1}^{2}, \vec{n}\right\rangle\right] \\
+\int_{\Omega}\left[\left\langle A \nabla a_{1}, \nabla a_{1}\right\rangle \phi_{x}^{2}-\left\langle A_{x} \nabla\left(a_{1}^{2} \phi_{x}\right), \nabla \phi\right\rangle+f_{v}(\phi, \psi) a_{1}^{2} \phi_{x} \psi_{x}\right] .
\end{aligned}
$$

Analogamente, a expressão do funcional $I$ calculado na função $a_{2} \phi_{y}$ é

$$
\begin{aligned}
\int_{\Omega} \Sigma_{y}=\int_{\partial \Omega}\left[\left\langle a_{2}^{2} \phi_{y} A_{y} \nabla \phi, \vec{n}\right\rangle-\frac{1}{2}\left\langle\phi_{y}^{2} A \nabla a_{2}^{2}, \vec{n}\right\rangle\right] \\
+\int_{\Omega}\left[\left\langle A \nabla a_{2}, \nabla a_{2}\right\rangle \phi_{y}^{2}-\left\langle A_{y} \nabla\left(a_{2}^{2} \phi_{y}\right), \nabla \phi\right\rangle+f_{v}(\phi, \psi) a_{2}^{2} \phi_{y} \psi_{y}\right] .
\end{aligned}
$$

Somando as expressões anteriores podemos indicar $\Sigma_{A}=I\left[a_{1} \phi_{x}\right]+I\left[a_{2} \phi_{y}\right]$ por

$$
\Sigma_{A}=I\left[a_{1} \phi_{x}\right]+I\left[a_{2} \phi_{y}\right]=\frac{1}{2} \int_{\partial \Omega} \frac{\partial}{\partial \nu}(\langle A \nabla \phi, \nabla \phi\rangle)+\int_{\Omega}\left(\Sigma_{x}+\Sigma_{y}\right),
$$

que, escrevemos

$$
\begin{aligned}
& \Sigma_{A}= \\
& \frac{1}{2} \int_{\partial \Omega}\left[\frac{\partial}{\partial \nu}(\langle A \nabla \phi, \nabla \phi\rangle)+2\left\langle\left(a_{1}^{2} \phi_{x} A_{x}+a_{2}^{2} \phi_{y} A_{y}\right) \nabla \phi, \vec{n}\right\rangle-\left\langle A\left(\phi_{x}^{2} \nabla a_{1}^{2}+\phi_{y}^{2} \nabla a_{2}^{2}\right), \vec{n}\right\rangle\right] \\
& +\int_{\Omega}\left[\left\langle A \nabla a_{1}, \nabla a_{1}\right\rangle \phi_{x}^{2}+\left\langle A \nabla a_{2}, \nabla a_{2}\right\rangle \phi_{y}^{2}-\left\langle A_{x} \nabla\left(a_{1}^{2} \phi_{x}\right)+A_{y} \nabla\left(a_{2}^{2} \phi_{y}\right), \nabla \phi\right\rangle\right] \\
& +\int_{\Omega} f_{v}(\phi, \psi)\langle A \nabla \phi, \nabla \psi\rangle .
\end{aligned}
$$


Comparando a expressão (3.13) com a expressão análoga (1.26) observamos a presença da parcela contendo o termo $f_{v}$ correspondente ao acoplamento do sistema (3.1). Comparando as expressões (2.23) e (3.13) percebemos a influência da dimensão $N$, da geometria do domínio, das propriedades analíticas dos coeficientes de difusividade e da derivada parcial $f_{v}$ no valor da expressão $\Sigma_{A}$ definida como a soma dos valores do funcional $I$ avaliado em funções adequadas. Portanto, a determinação da estabilidade ou da instabilidade do equilíbrio $\phi$ do sistema parcial (3.11), feita a partir da análise do sinal da expressão (3.13), depende de todas essas características, o que não ocorre quando se aplica o mesmo método para estudar a instabilidade de equilíbrios das equações com coeficientes constantes.

Consideremos

$$
I_{A}=\int_{\Omega}\left[\left\langle A \nabla a_{1}, \nabla a_{1}\right\rangle \phi_{x}^{2}+\left\langle A \nabla a_{2}, \nabla a_{2}\right\rangle \phi_{y}^{2}-\left\langle A_{x} \nabla\left(a_{1}^{2} \phi_{x}\right)+A_{y} \nabla\left(a_{2}^{2} \phi_{y}\right), \nabla \phi\right\rangle\right]
$$

a segunda integral da expressão $\Sigma_{A}$ dada em (3.13). De maneira análoga à usada para representar a segunda integral, $I$, da soma (1.26), vamos re-escrever a integral $I_{A}$ de maneira conveniente, de modo a usar as propriedades tanto geométricas quanto analíticas das funções difusividades $a_{1}$ e $a_{2}$. Para tanto, consideremos as seguintes definições $A \Delta a_{1}=a_{1}^{2}\left(a_{1}\right)_{x x}+a_{2}^{2}\left(a_{1}\right)_{y y}, A \Delta a_{2}=a_{1}^{2}\left(a_{2}\right)_{x x}+a_{2}^{2}\left(a_{2}\right)_{y y}$ e a forma quadrática $\langle\mathbb{A} \xi, \xi\rangle, \xi \in \mathbb{R}^{2}$, com a matriz $\mathbb{A}=\mathbb{A}(x, y),(x, y) \in \Omega$, dada por

$$
\mathbb{A}=\left(\begin{array}{cc}
a_{2}^{2}\left(a_{1}\right)_{y}^{2}+a_{1}\left(a_{1}\right)_{y} a_{2}\left(a_{2}\right)_{y} & -2 a_{1}\left(a_{1}\right)_{y} a_{2}\left(a_{2}\right)_{x} \\
-2 a_{1}\left(a_{1}\right)_{y} a_{2}\left(a_{2}\right)_{x} & a_{1}^{2}\left(a_{2}\right)_{x}^{2}+a_{1}\left(a_{1}\right)_{x} a_{2}\left(a_{2}\right)_{x}
\end{array}\right)
$$

Temos, assim, a seguinte expressão alternativa para a integral $I_{A}$ de (3.13)

$$
I_{A}=-\int_{\partial \Omega}\left[a_{1} \phi_{x}^{2} \frac{\partial a_{1}}{\partial \nu}+a_{2} \phi_{y}^{2} \frac{\partial a_{2}}{\partial \nu}\right] \mathrm{d} \sigma+\int_{\Omega}\left[a_{1} \phi_{x}^{2} A \Delta a_{1}+a_{2} \phi_{y}^{2} A \Delta a_{2}\right]+2 \int_{\Omega}\langle\mathbb{A} \nabla \phi, \nabla \phi\rangle
$$

Notamos que para a análise do sinal da expressão (3.15) é necessário levar em consideração as características das funções difusividade. Quando o domínio $\Omega$ é convexo a determinação do sinal da expressão (3.13) só depende de $I_{A}$ e da integral envolvendo a 
derivada parcial $f_{v}$. Por comodidade, indicamos

$$
\begin{aligned}
& \Sigma_{A}= \\
& \frac{1}{2} \int_{\partial \Omega}\left[\frac{\partial}{\partial \nu}(\langle A \nabla \phi, \nabla \phi\rangle)+2\left\langle\left(a_{1}^{2} \phi_{x} A_{x}+a_{2}^{2} \phi_{y} A_{y}\right) \nabla \phi, \vec{n}\right\rangle-\left\langle A\left(\phi_{x}^{2} \nabla a_{1}^{2}+\phi_{y}^{2} \nabla a_{2}^{2}\right), \vec{n}\right\rangle\right] \\
& -\int_{\partial \Omega}\left[a_{1} \phi_{x}^{2} \frac{\partial a_{1}}{\partial \nu}+a_{2} \phi_{y}^{2} \frac{\partial a_{2}}{\partial \nu}\right] \mathrm{d} \sigma+\int_{\Omega}\left[a_{1} \phi_{x}^{2} A \Delta a_{1}+a_{2} \phi_{y}^{2} A \Delta a_{2}\right]+2 \int_{\Omega}\langle\mathbb{A} \nabla \phi, \nabla \phi\rangle \\
& +\int_{\Omega} f_{v}(\phi, \psi)\langle A \nabla \phi, \nabla \psi\rangle .
\end{aligned}
$$

Pela expressão (3.16), podemos enunciar o seguinte resultado a respeito da instabilidade de quilíbrios não-constantes do problema parcial (3.11).

Teorema 3.2.1 Sejam $\Omega$ um domínio limitado convexo do plano com bordo $\partial \Omega$, curva de classe $\mathscr{C}^{2} e(\phi, \psi)$ equilibrio de (3.1) de modo que $u=\phi$ seja um equilibrio de (3.11). Considere que a matriz de difusividade $A: \Omega \rightarrow \mathbb{M}_{2}(\mathbb{R})$ seja uma matriz diagonal satisfazendo $A \Delta a_{1}<0$ e $A \Delta a_{2}<0$ em $\Omega, \frac{\partial a_{1}}{\partial \nu} \geq 0$ e $\frac{\partial a_{2}}{\partial \nu} \geq 0$ em $\partial \Omega$ e que a forma quadrática $\langle\mathbb{A} \xi, \xi\rangle$, definida pela matriz $\mathbb{A}$, dada em (3.14), satisfaça $\langle\mathbb{A} \xi, \xi\rangle \leq 0$ em $\Omega$ para todo $\xi \in \mathbb{R}^{2}$. Nessas condições, se $\phi$ for um equilibrio não-constante do problema (3.11) e se $\left|f_{v}(u, v)\right|$ for suficientemente pequeno num conjunto limitado de $\mathbb{R}^{2}$, então $\phi$ será instável.

Demonstração. A demonstração segue a mesma linha de raciocínio usada para provar o Teorema 2.2.2 e é esboçada como segue. Consideremos a expressão $\Sigma_{A}=I\left[a_{1} \phi_{x}\right]+$ $I\left[a_{2} \phi_{y}\right]$ dada em (3.16). Como o domínio $\Omega$ é convexo, pelo Teorema 1.2.1, temos que a integral sobre $\partial \Omega$ da expressão (3.13) é menor que ou igual a zero. Resta decidir os sinais das duas outras integrais. Quanto à integral $I_{A}$, da expressão (3.15) e, pelas hipóteses admitidas, temos que $I_{A}$ é estritamente menor que zero. Finalmente, da condição sobre $f_{v}$ num limitado de $\mathbb{R}^{2}$ concluimos que vale

$$
\Sigma_{A}=I\left[a_{1} \phi_{x}\right]+I\left[a_{2} \phi_{y}\right] \leq 0
$$

Dessa desigualdade, por um argumento análogo ao usado para demonstrar o Teorema 1.4.1, segue a instabilidade de $\phi$ como equilíbrio do problema parcial (3.11).

Ainda considerando fixado o equilíbrio $(\phi, \psi)$ do sistema (3.1), seja o sistema parcial 
obtido substituindo-se a função $u=\phi(x)$ na segunda equação do sistema (3.1) dado por

$$
\left\{\begin{aligned}
S v_{t} & =\operatorname{div}(B(x) \nabla v)+g(\phi(x), v), \quad x \in \Omega, \quad t>0 \\
\frac{\partial v}{\partial \nu} & =0, \quad x \in \partial \Omega, \quad t>0
\end{aligned}\right.
$$

Note que $v=\psi$ é um equilíbrio do sistema parcial (3.17).

O sistema linearizado em torno do equilíbrio $\psi$ é

$$
\left\{\begin{aligned}
S V_{t} & =\operatorname{div}(B(x) \nabla V)+g_{v}(\phi, \psi(x)) V, \quad x \in \Omega, \quad t>0 \\
\frac{\partial V}{\partial \nu} & =0, \quad x \in \partial \Omega, \quad t>0
\end{aligned}\right.
$$

com o operador linear $\mathscr{L}_{2}$ definido em $\left(\mathbf{H}^{2}(\Omega)\right)$ por

$$
\mathscr{L}_{2}[v]=-\operatorname{div}(B(x) \nabla v)-g_{v}(\phi, \psi) v, \quad \operatorname{com} \quad \frac{\partial v}{\partial \nu}=0 \quad \text { em } \quad \partial \Omega
$$

Para avaliar o sinal do autovalor principal do operador linear $\mathscr{L}_{2}$ consideremos o funcional quạdrático $J:\left(\mathbf{H}^{1}(\Omega)\right) \longrightarrow \mathbb{R}$ dado por

$$
J[v]=\int_{\Omega}\left[\langle B \nabla v, \nabla v\rangle-g_{v}(\phi, \psi) v^{2}\right] \mathrm{d} x, \quad \forall v \in \mathbf{H}^{1}(\Omega) .
$$

Se $\psi$ é equilíbrio não-constante de (3.17) calculando o funcional $J$ nas funções $v=b_{j} \psi_{x_{j}}$, $1 \leq j \leq N$, temos

$$
J\left[b_{j} \psi_{x_{j}}\right]=\int_{\Omega}\left[\left\langle B(x) \nabla\left(B_{j} \psi_{x_{j}}\right), \nabla\left(b_{j} \psi_{x_{j}}\right)\right\rangle-g_{v}(\phi, \psi)\left(b_{j} \psi_{x_{j}}\right)^{2}\right] \mathrm{d} x
$$

Como fizemos anteriormente no caso do sistema (3.8) vamos considerar o sistema (3.17) em dimensão $N=2$ definido num domínio limitado $\Omega$ do plano dado por.

$$
\left\{\begin{aligned}
v_{t} & =\left(b_{1}^{2}(x, y) v_{x}\right)_{x}+\left(b_{2}^{2}(x, y) v_{y}\right)_{y}+g(\phi, v), \quad(x, y) \in \Omega, \quad t>0 \\
\frac{\partial v}{\partial \nu} & =b_{1}^{2} v_{x} n_{1}+b_{2}^{2} v_{y} n_{2}=0, \quad(x, y) \in \partial \Omega, \quad t \geq 0
\end{aligned}\right.
$$

Nessas condições, temos as seguintes expressões para o funcional $J$ :

$$
J\left[b_{1} \psi_{x}\right]=\int_{\partial \Omega}\left\langle B(x) \nabla\left(b_{1} \psi_{x}\right), \nabla\left(b_{1} \psi_{x}\right)\right\rangle-g_{v}(\phi, \psi)\left(b_{1} \psi_{x}\right)^{2}
$$

e

$$
J\left[b_{2} \psi_{y}\right]=\int_{\partial \Omega}\left\langle B(x) \nabla\left(b_{2} \psi_{y}\right), \nabla\left(b_{2} \psi_{y}\right)\right\rangle-g_{v}(\phi, \psi)\left(b_{2} \psi_{y}\right)^{2}
$$


De maneira análoga ao caso do sistema parcial (3.11), consideremos a seguinte expressão $\Sigma_{B}=J\left[b_{1} \psi_{x}\right]+J\left[b_{2} \psi_{y}\right]$ :

$$
\begin{array}{r}
\Sigma_{B}= \\
\begin{array}{r}
\frac{1}{2} \int_{\partial \Omega}\left[\frac{\partial}{\partial \nu}(\langle B \nabla \psi, \nabla \psi\rangle)+2\left\langle\left(b_{1}^{2} \psi_{x} B_{x}+b_{2}^{2} \psi_{y} B_{y}\right) \nabla \psi, \vec{n}\right\rangle-\left\langle B\left(\psi_{x}^{2} \nabla b_{1}^{2}+\psi_{y}^{2} \nabla b_{2}^{2}\right), \vec{n}\right\rangle\right] \\
+\int_{\Omega}\left[\left\langle B \nabla b_{1}, \nabla b_{1}\right\rangle \psi_{x}^{2}+\left\langle B \nabla b_{2}, \nabla b_{2}\right\rangle \psi_{y}^{2}-\left\langle B_{x} \nabla\left(b_{1}^{2} \psi_{x}\right)+B_{y} \nabla\left(b_{2}^{2} \psi_{y}\right), \nabla \psi\right\rangle\right] \\
+\int_{\Omega} g_{u}(\phi, \psi)\langle\nabla \phi, B \nabla \psi\rangle .
\end{array}
\end{array}
$$

Analogamente ao caso do problema (3.11) o estudo da instabilidade do equilíbrio $\psi$ do sistema (3.20) será feito analisando o sinal da expressão (3.21). A segunda integral em (3.21) pode ser indicada por $I_{B}$ dada por

$$
I_{B}=-\int_{\partial \Omega}\left[b_{1} \psi_{x}^{2} \frac{\partial b_{1}}{\partial \nu}+b_{2} \psi_{y}^{2} \frac{\partial b_{2}}{\partial \nu}\right] \mathrm{d} \sigma+\int_{\Omega}\left[b_{1} \psi_{x}^{2} B \Delta b_{1}+b_{2} \psi_{y}^{2} B \Delta b_{2}\right]+2 \int_{\Omega}\langle\mathbb{B} \nabla \psi, \nabla \psi\rangle
$$

onde estamos considerando as seguintes expressões $B \Delta b_{1}=b_{1}^{2}\left(b_{1}\right)_{x x}+b_{2}^{2}\left(b_{1}\right)_{y y}, B \Delta b_{2}=$ $b_{1}^{2}\left(b_{2}\right)_{x x}+b_{2}^{2}\left(b_{2}\right)_{y y}$ e a forma quadrática $\langle\mathbb{B} \chi, \chi\rangle, \chi \in \mathbb{R}^{2}$, com a matriz $\mathbb{B}=\mathbb{B}(x, y)$, $(x, y) \in \Omega$, dada por

$$
\mathbb{B}=\left(\begin{array}{cc}
b_{2}^{2}\left(b_{1}\right)_{y}^{2}+b_{1}\left(b_{1}\right)_{y} b_{2}\left(b_{2}\right)_{y} & -2 b_{1}\left(b_{1}\right)_{y} b_{2}\left(b_{2}\right)_{x} \\
-2 b_{1}\left(b_{1}\right)_{y} b_{2}\left(b_{2}\right)_{x} & b_{1}^{2}\left(b_{2}\right)_{x}^{2}+b_{1}\left(b_{1}\right)_{x} b_{2}\left(b_{2}\right)_{x}
\end{array}\right)
$$

Vemos que, para sistemas definidos em domínios convexos, a determinação do sinal da expressão $\Sigma_{B}$ depende apenas dos sinais da expressão (3.22) e da integral contendo o termo $g_{u}$. Os cálculos acima permitem indicar

$$
\begin{array}{r}
\Sigma_{B}= \\
\frac{1}{2} \int_{\partial \Omega}\left[\frac{\partial}{\partial \nu}(\langle B \nabla \psi, \nabla \psi\rangle)+2\left\langle\left(b_{1}^{2} \psi_{x} B_{x}+b_{2}^{2} \psi_{y} B_{y}\right) \nabla \psi, \vec{n}\right\rangle-\left\langle B\left(\psi_{x}^{2} \nabla b_{1}^{2}+\psi_{y}^{2} \nabla b_{2}^{2}\right), \vec{n}\right\rangle\right] \\
-\int_{\partial \Omega}\left[b_{1} \psi_{x}^{2} \frac{\partial b_{1}}{\partial \nu}+b_{2} \psi_{y}^{2} \frac{\partial b_{2}}{\partial \nu}\right] \mathrm{d} \sigma+\int_{\Omega}\left[b_{1} \psi_{x}^{2} B \Delta b_{1}+b_{2} \psi_{y}^{2} B \Delta b_{2}\right]+2 \int_{\Omega}\langle\mathbb{B} \nabla \psi, \nabla \psi\rangle \\
+\int_{\Omega} g_{u}(\phi, \psi)\langle\nabla \phi, B \nabla \psi\rangle .
\end{array}
$$

Para o sistema parcial (3.20), temos um resultado análogo ao Teorema 3.2.1, referente à instabilidade de equilíbrios não-constantes que decorre da expressão (3.24), a saber 
Teorema 3.2.2 Sejam $\Omega$ um dominio limitado convexo do plano com bordo $\partial \Omega$, curva de classe $\mathscr{C}^{2} e(\phi, \psi)$ equilibrio de (3.1) de modo que $v=\psi$ seja um equilibrio de (3.20). Considere que a matriz de difusividade $B: \Omega \rightarrow \mathbb{M}_{2}(\mathbb{R})$ seja uma matriz diagonal satisfazendo $B \Delta b_{1}<0$ e $B \Delta b_{2}<0$ em $\Omega, \frac{\partial b_{1}}{\partial \nu} \geq 0$ e $\frac{\partial b_{2}}{\partial \nu} \geq 0$ em $\partial \Omega$ e que a forma quadrática $\langle\mathbb{B} \chi, \chi\rangle$, definida pela matriz $\mathbb{B}$, dada em (3.23), satisfaça $\langle\mathbb{B} \chi, \chi\rangle \leq 0$ em $\Omega$ para todo $\chi \in \mathbb{R}^{2}$. Nessas condições, se $\psi$ for um equilíbrio não-constante do problema (3.20) e se $\left|g_{u}(u, v)\right|$ for suficientemente pequeno num conjunto limitado de $\mathbb{R}^{2}$, então $\psi$ será instável.

\subsection{O Sistema Original}

Um fato sobre a estabilidade do equilíbrio $(\phi, \psi)$ do sistema (3.1) que decorre diretamente da expressão (3.7) e cuja demonstração é, essencialmente, a mesma dada no caso análogo para os sistema unidimensionais do Capítulo 2 é a proposição seguinte.

Proposição 3.3.1 Seja $(\phi, \psi)$ equilíbrio de (3.1) tal que $\phi$ é um equilíbrio estável de (3.8) e $\psi$ um equilibrio estável de (3.17). Nessas condições, se o sistema é anti-gradiente, então $(\phi, \psi)$ é estável.

Entretanto, conforme mostra o Exemplo (3.28), não se pode afirmar a instabilidade de um equilíbrio $(\phi, \psi)$ do sistema (3.1) simplesmente sabendo-se que $\phi$ e $\psi$ são instáveis como equilíbrios dos respectivos sistemas parciais. Podemos enunciar um resultado análogo ao Teorema 2.3.2, como segue.

Teorema 3.3.1 Seja $(\phi, \psi)$ uma solução do sistema (3.2) de modo que, para quaisquer $R$ e $S$, o par $(\phi, \psi)$ seja um equilibrio do sistema anti-gradiente (3.1). Se a função $\phi$ for um equilíbrio instável do sistema parcial

$$
\left\{\begin{array}{l}
u_{t}=\operatorname{div}(A(x) \nabla u)+f(u, \psi), x \in \Omega, t>0 \\
\frac{\partial u}{\partial \nu}=0, \quad x \in \partial \Omega, \quad t>0
\end{array}\right.
$$

correspondente, então podemos determinar $\Gamma>0$ de modo que $(\phi, \psi)$ seja instável como 
equilibrio de

$$
\left\{\begin{aligned}
u_{t} & =\operatorname{div}(A(x) \nabla u)+f(u, v), x \in \Omega, t>0 \\
\Gamma v_{t} & =\operatorname{div}(B(x) \nabla v)+g(u, v), x \in \Omega, t>0 \\
\frac{\partial u}{\partial \nu} & =\frac{\partial u}{\partial \nu}=0, \quad x \in \partial \Omega, \quad t>0
\end{aligned}\right.
$$

Para obter alguma informação sobre a instabilidade do equilíbrio $(\phi, \psi)$ do problema (3.1) vamos combinar as expressões (3.16) e (3.24), de modo a afirmar um resultado análogo ao Teorema 2.3.3. Com esse objetivo, efetuemos alguns cálculos preliminares. Consideremos, portanto,

$$
\Sigma=\Sigma_{A}+\Sigma_{B}=I\left[a_{1} \phi_{x}\right]+I\left[a_{2} \phi_{y}\right]+J\left[b_{1} \psi_{x}\right]+J\left[b_{2} \psi_{y}\right]
$$

Somando as parcelas das expressões (3.16) e (3.24) correspondentes aos integrandos contendo $f_{v}$ e $g_{u}$ obtemos

$$
\int_{\Omega} f_{v}(\phi, \psi)\langle A \nabla \phi, \nabla \psi\rangle+g_{u}(\phi, \psi)\langle\nabla \phi, B \nabla \psi\rangle=\int_{\Omega}\left\langle\left(f_{v}(\phi, \psi) A+g_{u}(\phi, \psi) B\right) \nabla \phi, \nabla \psi\right\rangle .
$$

Pela condição de anti-gradiente do sistema (3.1) dado, temos $g_{u}=-f_{v}$, donde

$$
\int_{\Omega} f_{v}(\phi, \psi)\langle A \nabla \phi, \nabla \psi\rangle+g_{u}(\phi, \psi)\langle B \nabla \phi, \nabla \psi\rangle=\int_{\Omega}\left\langle f_{v}(\phi, \psi)(A-B) \nabla \phi, \nabla \psi\right\rangle .
$$

Em particular, se as matrizes dos coeficientes de difusividade forem iguais, então, de (3.25) vem que a soma das últimas integrais de (3.16) e (3.24), é zero e a expressão $\Sigma=\Sigma_{A}+\Sigma_{B}$ reduz-se a expressões que envolvem conhecimentos sobre o comportamento das funções difusividades em $\Omega$ e em $\partial \Omega$ cujos resultados já são conhecidos do Capítulo 1 . Por outro lado, se $\left|f_{v}(u, v)\right|=\left|g_{u}(u, v)\right|$ for suficientemente pequeno num subconjunto limitado de $\mathbb{R}^{2}$ ou se, para todo $x \in \Omega$, a diferença $A(x)-B(x)$ for suficientemente pequena, então a influência da integral $\int_{\Omega}\left\langle f_{v}(A-B) \nabla \phi, \nabla \psi\right\rangle$ na soma $\Sigma$ acima também será pequena.

\subsubsection{Caso $A=B$}

Indiquemos $A=\operatorname{diag}\left\{a^{2}, b^{2}\right\}$, de modo que o sistema (3.1) é dado particularmente por

$$
\left\{\begin{aligned}
R u_{t} & =\operatorname{div}(A(x, y) \nabla u)+f(u, v), \quad(x, y) \in \Omega, \quad t>0 \\
S v_{t} & =\operatorname{div}(A(x, y) \nabla v)+g(u, v), \quad(x, y) \in \Omega, \quad t>0 \\
\frac{\partial u}{\partial \nu} & =\frac{\partial v}{\partial \nu}=0, \quad(x, y) \in \partial \Omega, \quad t>0
\end{aligned}\right.
$$


Desse modo, a soma $\Sigma$ é expressa por

$$
\begin{aligned}
& \Sigma= \\
= & \Sigma_{A}+\Sigma_{B}=I\left[a \phi_{x}\right]+I\left[b \phi_{y}\right]+J\left[a \psi_{x}\right]+J\left[b \psi_{y}\right] \\
= & \frac{1}{2} \int_{\partial \Omega}\left[\frac{\partial}{\partial \nu}(\langle A \nabla \phi, \nabla \phi\rangle)+2\left\langle\left(a^{2} \phi_{x} A_{x}+b^{2} \phi_{y} A_{y}\right) \nabla \phi, \vec{n}\right\rangle-\left\langle A\left(\phi_{x}^{2} \nabla a^{2}+\phi_{y}^{2} \nabla b^{2}\right), \vec{n}\right\rangle\right] \\
+ & \frac{1}{2} \int_{\partial \Omega}\left[\frac{\partial}{\partial \nu}(\langle A \nabla \psi, \nabla \psi\rangle)+2\left\langle\left(a^{2} \psi_{x} A_{x}+b^{2} \psi_{y} A_{y}\right) \nabla \psi, \vec{n}\right\rangle-\left\langle A\left(\psi_{x}^{2} \nabla a^{2}+\psi_{y}^{2} \nabla b^{2}\right), \vec{n}\right\rangle\right] \\
+ & I_{A}+I_{B}
\end{aligned}
$$

onde estamos considerando

$$
I_{A}=-\int_{\partial \Omega}\left[a \phi_{x}^{2} \frac{\partial a}{\partial \nu}+b \phi_{y}^{2} \frac{\partial b}{\partial \nu}\right] \mathrm{d} \sigma+\int_{\Omega}\left[a \phi_{x}^{2} A \Delta a+b \phi_{y}^{2} A \Delta b\right]+2 \int_{\Omega}\langle\mathbb{A} \nabla \phi, \nabla \phi\rangle
$$

e

$$
I_{B}=-\int_{\partial \Omega}\left[a \psi_{x}^{2} \frac{\partial a}{\partial \nu}+b \psi_{y}^{2} \frac{\partial b}{\partial \nu}\right] \mathrm{d} \sigma+\int_{\Omega}\left[a \psi_{x}^{2} A \Delta a+b \psi_{y}^{2} A \Delta b\right]+2 \int_{\Omega}\langle\mathbb{A} \nabla \psi, \nabla \psi\rangle
$$

Nessas condições, podemos afirmar o seguinte resultado.

Teorema 3.3.2 Seja $\Omega$ um domínio limitado convexo do plano $\mathbb{R}^{2}$ com bordo suave $e$ considere o sistema (3.26) com estrutura anti-gradiente com a matriz de difusividades $A$ satisfazendo $A \Delta a \leq 0, A \Delta b \leq 0$ em $\Omega, \frac{\partial a}{\partial \nu} \geq 0$ e $\frac{\partial b}{\partial \nu} \geq 0$ em $\partial \Omega$ com a forma quadrática $\langle\mathbb{A} \xi, \xi\rangle$ definida pela matriz $\mathbb{A}$, dada em (1.28), satisfazendo $\langle\mathbb{A} \xi, \xi\rangle \leq 0$ em $\Omega$ para todo $\xi \in \mathbb{R}^{2}$. Nessas circunstâncias, se $(\phi, \psi)$ é um equilibrio não-constante do sistema (3.26), então, $\lambda^{u}<0$ ou $\lambda^{v}<0$.

Demonstração. A demonstração do Teorema segue a mesma linha de reciocínio usada para demonstrar o Teorema 2.3.3 baseando-se na análise do sinal da expressão $\Sigma$ dada em (3.27). Da convexidade do domínio $\Omega$ segue que as duas integrais sobre $\partial \Omega$ são menores que ou iguais a zero. Com as hipóteses sobre os coeficientes de difusividade temos $I_{A} \leq 0$ e $I_{B} \leq 0$. Desse modo temos $\Sigma \leq 0$, donde segue o resultado.

Corolário 3.3.1 Seja $\Omega$ um dominio limitado convexo do plano $\mathbb{R}^{2}$ com bordo suave $e$ considere o sistema (3.26) com estrutura anti-gradiente e com a matriz de difusividades satisfazendo $A \Delta a \leq 0, A \Delta b \leq 0$ em $\Omega, \frac{\partial a}{\partial \nu} \geq 0$ e $\frac{\partial b}{\partial \nu} \geq 0$ em $\partial \Omega$ com a forma quadrática $\langle\mathbb{A} \xi, \xi\rangle$ definida pela matriz $\mathbb{A}$ dada em (1.28), satisfazendo $\langle\mathbb{A} \xi, \xi\rangle \leq 0$ em $\Omega$ para todo 
$\xi \in \mathbb{R}^{2}$. Nessas circunstâncias, se $(\phi, \psi)$ é uma solução não-constante do sistema elíptico (3.2), com $A=B$, de modo que $\phi$ seja um equilíbrio instável do sistema parcial (3.11), então, existe $\Gamma>0$ suficientemente grande tal que $(\phi, \psi)$ seja instável como equilíbrio do sistema (3.26).

Demonstração. Das hipóteses, pela expressão (3.27), temos $\Sigma \leq 0$, donde, pelo Teorema 3.3.2, temos $\lambda^{u}<0$ ou $\lambda^{v}<0$. Das hipóteses sabemos ainda que $\phi$ é instável como equilíbrio de (3.11), donde vale $\lambda^{u}<0$. Pelo Teorema 3.3.1, podemos escolher $\Gamma>0$ de modo que $(\phi, \psi)$ seja instável como equilíbrio do sistema (3.26).

Podemos enunciar um outro fato sobre a instabilidade de equilíbrios não-constantes do sistema (3.1), a saber,

Corolário 3.3.2 Com as mesmas hipóteses do Corolário anterior, se $(\phi, \psi)$ é uma solução não-constante do sistema elíptico (3.2), com $A=B$, então, existem constantes $R>0$ e $S>0$ tais que $(\phi, \psi)$ é um equilíbrio não-constante instável do sistema (3.1).

Para uma classe particular de domínios planos convexos e para uma família especial de sistemas com difusividades variáveis e com estrutura anti-gradiente podemos enunciar resultados análogos aos enunciados no caso das equações. Como aplicações dos resultados que vimos anteriormente, podemos obter alguns exemplos de sistemas com coeficientes variáveis, definidos em convexos especiais do plano, para os quais valem as conclusões do Corolário 3.3.1.

Corolário 3.3.3 Consideremos um domínio limitado convexo $\Omega$ dado por

$$
\Omega=\left\{(x, y) \in \mathbb{R}^{2} ; f_{1}(x)<y<f_{2}(x) ; c<x<d\right\}
$$

onde $f_{1}, f_{2}:[c, d] \rightarrow \mathbb{R}$ são funções de classe $\mathscr{C}^{2}$. Seja $a:[c, d] \rightarrow \mathbb{R}$ de classe $\mathscr{C}^{2}$ tal que $a^{\prime \prime}(x)<0, a^{\prime}(x) f_{2}^{\prime}(x) \leq 0$ e $a^{\prime}(x) f_{1}^{\prime}(x) \geq 0$ para todo $x$ em $[c, d]$. Seja $(\phi, \psi)$ uma solução não-constante do problema elíptico (3.2), com $A=B$, Nessas condições, se $\left|f_{v}\right|$ for suficientemente pequeno num subconjunto limitado de $\mathbb{R}^{2}$, então $\Gamma>0$ pode ser escolhido de modo que $(\phi, \psi)$ seja um equilibrio não-constante instável do sistema 
anti-gradiente

$$
\left\{\begin{aligned}
u_{t} & =\left(a^{2}(x) u_{x}\right)_{x}+u_{y y}+f(u, v), \quad(x, y) \in \Omega, t>0 \\
\Gamma v_{t} & =\left(a^{2}(x) v_{x}\right)_{x}+v_{y y}+g(u, v), \quad(x, y) \in \Omega, t>0 \\
\frac{\partial u}{\partial \nu} & =a^{2}(x) u_{x} n_{1}+u_{y} n_{2}=0, \quad(x, y) \in \partial \Omega, t>0 \\
\frac{\partial v}{\partial \nu} & =a^{2}(x) v_{x} n_{1}+v_{y} n_{2}=0, \quad(x, y) \in \partial \Omega, t>0 .
\end{aligned}\right.
$$

Demonstração. Conforme nos indica a expressão (3.7), o sinal do autovalor principal do linearizado do sistema em torno do equilíbrio $(\phi, \psi)$ pode ser decidido pela soma $\Sigma=\Sigma_{A}+\Sigma_{B}$, dada em (3.27). Da convexidade do domínio temos que as integrais sobre $\partial \Omega$ da expressão (3.27) são menores que ou iguais a zero. Portanto, resta decidir o sinal das integrais $I_{A}$ e $I_{B}$. Notamos que, para a análise do sinal das integrais restantes, procedemos de maneira análoga à demonstração do Teorema 1.4.1. De fato, basta observar que, quanto à parcela $I_{A}$, pelo uso das hipóteses, temos

$$
\begin{aligned}
I_{A} & =\int_{\Omega}\left[a a_{x x}\left(a \phi_{x}\right)^{2}-\frac{\partial}{\partial x}\left(a a_{x}\left(a \phi_{x}\right)^{2}\right)\right] \mathrm{d} x \\
& =\int_{\Omega} a\left(\phi_{x}\right)^{2} a^{2} a_{x x} \mathrm{~d} x-\int_{\partial \Omega} a\left(\phi_{x}\right)^{2} a^{2} a_{x} n_{1} \mathrm{~d} \sigma \leq 0
\end{aligned}
$$

e, quanto à parcela $I_{B}$, com as hipóteses obtemos

$$
I_{B}=\quad \int_{\Omega} a\left(\psi_{x}\right)^{2} a^{2} a_{x x} \mathrm{~d} x-\int_{\partial \Omega} a\left(\psi_{x}\right)^{2} a^{2} a_{x} n_{1} \mathrm{~d} \sigma \leq 0 .
$$

Como $a^{\prime \prime}(x)<0$ em $\Omega$ e pelas condições da difusividade $a$ sobre o bordo $\partial \Omega$ temos que $\Sigma=I\left[a \phi_{x}\right]+I\left[\phi_{y}\right]+J\left[a \psi_{x}\right]+J\left[\psi_{y}\right] \leq 0$. Pelo Teorema 3.3 .2 temos $\lambda^{u}<0$ ou $\lambda^{v}<0$. Da condição $\left|f_{v}\right|$ suficientemente pequena podemos concluir que $\phi$ é um equilíbrio instável do problema parcial correspondente. A conclusão decorre, agora, de uma aplicação do Corolário 3.3.1.

Um outro exemplo de sistema com coeficientes de difusão variáveis definido num domínio convexo particular do plano para o qual vale a conclusão do Corolário 3.3.1 é o seguinte

Corolário 3.3.4 Seja $g:[c, d] \rightarrow[p, q]$ uma função de classe $\mathscr{C}^{2}$ tal que $g^{\prime}>0$ em $(c, d)$ e seja $h:[p, q] \rightarrow[c, d]$ sua inversa. Consideremos um domínio limitado convexo $\Omega$ dado por

$$
\Omega=\left\{(x, y) \in \mathbb{R}^{2} ; c<x<d, \quad p<y<g(x)\right\} .
$$


Sejam $a:[c, d] \rightarrow \mathbb{R}$ e $b:[p, q] \rightarrow \mathbb{R}$ de classe $\mathscr{C}^{2}$ tais que $a^{\prime \prime}(x) \leq 0$ em $[c, d]$ e $b^{\prime \prime}(y) \leq 0$ em $[p, q]$ e, além disso, $a^{\prime} g^{\prime} \leq 0$ e $b^{\prime} h^{\prime} \leq 0$ em seus respectivos domínios. Seja $(\phi, \psi)$ uma solução não-constante do problema elíptico (3.2), com $A=B$. Se $\left|f_{v}\right|$ for suficientemente pequeno num subconjunto limitado do plano então, existe $\Gamma>0$ tal que $(\phi, \psi)$ é um equilíbrio não-constante instável do sistema anti-gradiente

$$
\left\{\begin{aligned}
u_{t} & =\left(a^{2}(x) u_{x}\right)_{x}+\left(b^{2}(y) u_{y}\right)_{y}+f(u, v), \quad(x, y) \in \Omega, t>0 \\
\Gamma v_{t} & =\left(a^{2}(x) v_{x}\right)_{x}+\left(b^{2}(y) v_{y}\right)_{y}+g(u, v), \quad(x, y) \in \Omega, t>0 \\
\frac{\partial u}{\partial \nu} & =a^{2}(x) u_{x} n_{1}+b^{2}(y) u_{y} n_{2}=0, \quad(x, y) \in \partial \Omega, t>0 \\
\frac{\partial v}{\partial \nu} & =a^{2}(x) v_{x} n_{1}+b^{2}(y) v_{y} n_{2}=0, \quad(x, y) \in \partial \Omega, t>0 .
\end{aligned}\right.
$$

\subsubsection{Caso $A \neq B$}

No caso em que $A(x, y) \neq B(x, y)$, pela expressão $\Sigma=\Sigma_{A}+\Sigma_{B}$ onde $\Sigma_{A}$ e $\Sigma_{B}$ são dados por (3.16) e (3.24), respectivamente, para que a soma das integrais contendo as derivadas parciais $f_{v}$ e $g_{u}$ não afetem os sinais das demais integrais da expressão, vamos exigir que $\left|f_{v}\right|$ seja suficientemente pequeno num subconjunto limitado do plano ou que $\|A-B\|$ seja suficientemente pequeno em $\Omega$. Nessas condições, ainda podemos enunciamos resultados análogos ao do Teorema 3.3.2, a saber,

Teorema 3.3.3 Seja $\Omega$ um domínio limitado convexo do plano $\mathbb{R}^{2}$ com bordo suave $e$ considere o sistema (3.1) com estrutura anti-gradiente com as matrizes de difusividades $A, B$ satisfazendo $A \Delta a_{1}<0, A \Delta a_{2}<0 \mathrm{em} \Omega, \frac{\partial a_{1}}{\partial \nu} \geq 0$ e $\frac{\partial a_{2}}{\partial \nu} \geq 0 \mathrm{em} \partial \Omega$ com a forma quadrática $\langle\mathbb{A} \xi, \xi\rangle$ definida pela matriz $\mathbb{A}$, dada em (3.14), satisfazendo $\langle\mathbb{A} \xi, \xi\rangle \leq 0$ em $\Omega$ para todo $\xi \in \mathbb{R}^{2}: B \Delta b_{1}<0, B \Delta b_{2}<0$ em $\Omega, \frac{\partial b_{1}}{\partial \nu} \geq 0$ e $\frac{\partial b_{2}}{\partial \nu} \geq 0$ em $\partial \Omega$ com a forma quadrática $\langle\mathbb{B} \chi, \chi\rangle$ definida pela matriz $\mathbb{B}$, dada em (3.23), satisfazendo $\langle\mathbb{B} \chi, \chi\rangle \leq 0$ em $\Omega$ para todo $\xi \in \mathbb{R}^{2}$. Nessas circunstâncias, se $\left|f_{v}\right|$ for suficientemente pequeno num limitado de $\mathbb{R}^{2}$ ou se $\|A-B\|$ for suficientemente pequeno em $\Omega$, e se $(\phi, \psi)$ é uma solução não-constante do sistema (3.1), então, $\lambda^{u}<0$ ou $\lambda^{v}<0$.

Demonstração. As hipóteses foram admitidas para que se tivesse $\Sigma=\Sigma_{A}+\Sigma_{B} \leq 0$ Das expressões (3.16) e (3.24) temos que as integrais sobre o bordo $\partial \Omega$ são menores que ou iguais a zero devido à convexidade do domínio. Pelas hipóteses, as integrais $I_{A} \mathrm{e}$ $I_{B}$ são estritamente negativas. Como o sistema é anti-gradiente temos $\left|f_{v}\right|=\left|g_{u}\right|$ e das 
hipóteses sobre $\left|f_{v}\right|$ ou sobre $\|A-B\|$ decorre $\Sigma \leq 0$. A conclusão segue de maneira análoga à do Teorema 3.3.2.

Corolário 3.3.5 Seja $(\phi, \psi)$ uma solução do sistema elíptico (3.2). Seja o problema (3.1) definido num domínio limitado $\Omega \subset \mathbb{R}^{N}$ convexo onde as matrizes de difusividades $A$ e $B$ satisfazem as condições $A \Delta a_{j} \leq 0, B \Delta b_{k} \leq 0$ em $\Omega$ e $\frac{\partial a_{j}}{\partial \nu}=0, \frac{\partial b_{k}}{\partial \nu}=0 \mathrm{em}$ $\partial \Omega$ para $1 \leq j \leq 2$ e $1 \leq k \leq 2,\langle\mathbb{A} \chi, \chi\rangle \leq 0$ e $\langle\mathbb{B} \xi, \xi\rangle \leq 0$ em $\Omega, \chi, \xi \in \mathbb{R}^{N}$. $f_{v}^{T}(\phi, \psi) A+B^{T} g_{u}(\phi, \psi)=0$ de modo que $\phi$ seja um equilibrio instável de (3.8). Nessas condições, existe $\Gamma>0$ tal que $(\phi, \psi)$ é um equilíbrio instável de (3.1).

Demonstração. A demonstração decorre de adaptação natural feita na demosntração do Corolário 3.3.1.

\subsection{Uma Classe de Exemplos}

Obtemos aqui uma família de exemplos de sistemas do tipo anti-gradiente com coeficientes constantes que admitem equilíbrios estáveis, embora os equilíbrios dos sistemas parciais correspondentes sejam instáveis. É obtida uma relação geométrica entre os coeficientes de modo que o sistema resultante tenha a propriedade desejada. Para tanto, consideremos o problema

$$
\left\{\begin{aligned}
\frac{\partial u}{\partial t} & =\Delta u+u+a v_{1}+b v_{2}, \quad x \in \Omega, \quad t>0 \\
\frac{\partial v_{1}}{\partial t} & =\Delta v_{1}-a u+c v_{1}, \quad x \in \Omega, \quad t>0 \\
\frac{\partial v_{2}}{\partial t} & =\Delta v_{2}-b u+d v_{2}, \quad x \in \Omega, \quad t>0 \\
\frac{\partial u}{\partial \nu} & =\frac{\partial v}{\partial \nu}=0, \quad x \in \partial \Omega, \quad t>0
\end{aligned}\right.
$$

No exemplo temos $f\left(u, v_{1}, v_{2}\right)=u+a v_{1}+b v_{2}$ e $g\left(u, v_{1}, v_{2}\right)=\left(-a u+c v_{1},-b u+d v_{2}\right)$, donde podemos considerar $H: \mathbb{R}^{3} \longrightarrow \mathbb{R}$ dada por $H\left(u, v_{1}, v_{2}\right)=\frac{1}{2} u^{2}-\frac{c}{2} v_{1}^{2}-\frac{d}{2} v_{2}^{2}+$ $u\left(a v_{1}+b v_{2}\right)$ de modo que temos $f=h_{u}$ e $g=h_{v}$.

Temos que $(0,0,0)$ é um equilíbrio do sistema. Vamos determinar algumas condições sobre os coeficientes $a, b, c, d$ para que o equilíbrio seja estável e que os equilíbrios 0 e $(0,0)$ dos sistemas parciais correspondentes sejam instáveis. 
Fixando $v=\left(v_{1}, v_{2}\right)=(0,0)$ na primeira equação temos

$$
\left\{\begin{array}{l}
\frac{\partial u}{\partial t}=\Delta u+u \quad x \in \Omega, \quad t>0 \\
\frac{\partial u}{\partial \nu}=0, \quad x \in \partial \Omega, \quad t>0
\end{array}\right.
$$

Temos que $u=0$ é um equilíbrio do sistema resultante. Considerando $\left(\mu_{k}\right)_{k \in \mathbb{N}}$ os autovalores do operador $-\Delta$ com condições de Neumann homogêneas na fronteira $\partial \Omega$ temos que os autovalores do linearizado em torno do equilíbrio $u=0$ são dados por $\lambda_{k}=\mu_{k}-1$. Como $\mu_{0}=0$ vem que $\lambda_{0}=-1$, donde segue que $u=0$ é equilíbrio instável.

Fixando $u=0$ nas demais equações do sistema dado temos

$$
\left\{\begin{aligned}
\frac{\partial v_{1}}{\partial t}=\Delta v_{1}+c v_{1}, & x \in \Omega, \quad t>0 \\
\frac{\partial v_{2}}{\partial t}=\Delta v_{2}+d v_{2}, & x \in \Omega, \quad t>0 \\
\frac{\partial v_{1}}{\partial \nu}=\frac{\partial v_{2}}{\partial \nu}=0, & x \in \partial \Omega, \quad t>0
\end{aligned}\right.
$$

É claro que $v=(0,0)$ é equilíbrio do sistema resultante. Considerando o problema de autovalores para o linearizado em torno do equilíbrio $v=(0,0)$ temos

$$
-\left(\begin{array}{c}
\Delta v_{1} \\
\Delta v_{2}
\end{array}\right)-\left(\begin{array}{ll}
c & 0 \\
0 & d
\end{array}\right)\left(\begin{array}{l}
v_{1} \\
v_{2}
\end{array}\right)=\lambda\left(\begin{array}{l}
v_{1} \\
v_{2}
\end{array}\right)
$$

Considerando $\left(\mu_{k}\right)_{k \in \mathbb{N}}$ os autovalores do operador $-\Delta$ com condições de Neumann homogêneas na fronteira $\partial \Omega$ temos que os autovalores do linearizado em torno do equilíbrio $v=(0,0)$ são dados por $\lambda_{k}=\mu_{k}-\xi$, onde $\xi$ é um autovalor da matriz $\left(\begin{array}{ll}c & 0 \\ 0 & d\end{array}\right)$. Como $\xi=c$ ou $\xi=d$ e $\mu_{0}=0$ vem que $\lambda_{0}=-c$ ou $\lambda_{0}=-d$ donde, para que $v=(0,0)$ seja instável devemos ter $c>0$ ou $d>0$.

Considerando o problema de autovalores para o linearizado em torno do equilíbrio $(0,0,0)$ do sistema dado temos

$$
-\left(\begin{array}{c}
\Delta u \\
\Delta v_{1} \\
\Delta v_{2}
\end{array}\right)-\left(\begin{array}{ccc}
1 & a & b \\
-a & c & 0 \\
-b & 0 & d
\end{array}\right)\left(\begin{array}{c}
u \\
v_{1} \\
v_{2}
\end{array}\right)=\lambda\left(\begin{array}{c}
u \\
v_{1} \\
v_{2}
\end{array}\right)
$$

com condições de Neumann homogêneas na fronteira. Queremos que os autovalores $\lambda_{k}$ satisfaçam $\operatorname{Re} \lambda_{k}>0, \forall k \in \mathbb{N}$. 
Considerando $\left(\mu_{k}\right)_{k \in \mathbb{N}}$ os autovalores do operador $-\Delta$ com condições de Neumann homogêneas na fronteira $\partial \Omega$ temos que os autovalores do linearizado em torno do equilíbrio $(0,0,0)$ são dados por $\lambda_{k}=\mu_{k}-\xi$, onde $\xi$ é um autovalor da matriz $A=\left(\begin{array}{ccc}1 & a & b \\ -a & c & 0 \\ -b & 0 & d\end{array}\right)$.

$$
\begin{gathered}
\operatorname{det}\left(\begin{array}{ccc}
\xi-1 & -a & -b \\
a & \xi-c & 0 \\
b & 0 & \xi-d
\end{array}\right)=0 \\
(\xi-1)(\xi-c)(\xi-d)+a^{2}(\xi-d)+b^{2}(\xi-c)=0 \\
\xi^{3}-(1+c+d) \xi^{2}+\left(a^{2}+b^{2}+c d+c+d\right) \xi-\left(a^{2} d+b^{2} c+c d\right)=0
\end{gathered}
$$

Impondo que -1 seja um autovalor da matriz $A$ obtemos a seguinte condição

$$
2[(1+c)(1+d)]+\left[a^{2}(1+d)+b^{2}(1+c)\right]=0 .
$$

Admitindo que sejam verificadas as condições $1+c \neq 0$ e $1+d \neq 0$ podemos escrever

$$
\frac{a^{2}}{1+c}+\frac{b^{2}}{1+d}=-2 \text {. }
$$

Observamos que a condição acima só não se verifica para $a, b$ reais se $1+c>0$ e $1+d>0$. Assim, se $1+c<0$ e $1+d<0 a, b$ pertencem à elipse

$$
\frac{a^{2}}{-2(1+c)}+\frac{b^{2}}{-2(1+d)}=1 \text {. }
$$

Se $1+c>0$ e $1+d<0 a, b$ pertencem à hipérbole

$$
\frac{b^{2}}{-2(1+d)}-\frac{a^{2}}{2(1+c)}=1 .
$$

Dividindo-se a expressão

$$
\xi^{3}-(1+c+d) \xi^{2}+\left(a^{2}+b^{2}+c d+c+d\right) \xi-\left(a^{2} d+b^{2} c+c d\right)
$$

por $\xi+1$ obtemos $\xi^{2}-(2+c+d) \xi^{2}+\left(a^{2}+b^{2}+c d+2 c+2 d+2\right)$. 
Impondo que a equação

$$
\xi^{2}-(2+c+d) \xi^{2}+\left(a^{2}+b^{2}+c d+2 c+2 d+2\right)=0
$$

tenha raízes complexas $\xi_{1}$ e $\xi_{2}$ com partes reais negativas devemos ter

$$
\operatorname{Re} \xi_{1}=\operatorname{Re} \xi_{2}=\frac{2+c+d}{2}<0,
$$

donde segue que $c+d<-2$.

Como as raízes devem ser complexas devemos ter

$$
(2+c+d)^{2}-4\left(a^{2}+b^{2}+c d+2 c+2 d+2\right)<0
$$

donde

$$
\left(\frac{c-d}{2}\right)^{2}-(c+d)<1+a^{2}+b^{2} .
$$

Como estamos admitindo $\xi_{1}=-1$, se adotarmos $\operatorname{Re} \xi_{1}=\operatorname{Re} \xi_{2}=-1$ teremos

$$
\frac{2+c+d}{2}=-1
$$

donde vem que $c+d=-4$.

Por exemplo, fazendo $c=1$ temos $d=-5$. Da expressão envolvendo $a$ e $b$ temos

$$
\frac{b^{2}}{8}-\frac{a^{2}}{4}=1
$$

Para $a=2$ obtemos $b= \pm 4$ e a matriz $A$ é dada por $A=\left(\begin{array}{ccc}1 & 2 & 4 \\ -2 & 1 & 0 \\ -4 & 0 & -5\end{array}\right)$ com os autovalores $\xi_{1}=-1, \xi_{2}=-1+i 2 \sqrt{2}$ e $\xi_{2}=-1-i 2 \sqrt{2}$

Assim, o sistema

$$
\left\{\begin{aligned}
\frac{\partial u}{\partial t} & =\Delta u+u+2 v_{1}+4 v_{2}, \quad x \in \Omega \quad t>0 \\
\frac{\partial v_{1}}{\partial t} & =\Delta v_{1}-2 u+v_{1}, \quad x \in \Omega, \quad t>0, \\
\frac{\partial v_{2}}{\partial t} & =\Delta v_{2}-4 u-5 v_{2}, \quad x \in \Omega, \quad t>0, \\
\frac{\partial u}{\partial \nu} & =\frac{\partial v}{\partial \nu}=0, \quad x \in \partial \Omega, \quad t>0,
\end{aligned}\right.
$$

possui estrutura anti-gradiente com $H\left(u, v_{1}, v_{2}\right)=\frac{1}{2} u^{2}-\frac{1}{2} v_{1}^{2}+\frac{5}{2} v_{2}^{2}+u\left(2 v_{1}+4 v_{2}\right)$ e admite $(0,0,0)$ como equilíbrio estável pois temos $\lambda_{k}=\mu_{k}+1, \lambda_{k}=\mu_{k}+1+i 2 \sqrt{2}$ ou 
$\lambda_{k}=\mu_{k}+1-i 2 \sqrt{2}, k=0,1,2, \ldots$ como os autovalores do linearizado correspondente. Entretanto, com relação aos sistemas "parciais" temos $u=0$ como equilíbrio instável de

$$
\left\{\begin{array}{l}
\frac{\partial u}{\partial t}=\Delta u+u, x \in \Omega, \quad t>0 \\
\frac{\partial u}{\partial \nu}=0, \quad x \in \partial \Omega, \quad t>0
\end{array}\right.
$$

e $\left(v_{1}, v_{2}\right)=(0,0)$ equilíbrio instável de

$$
\left\{\begin{array}{l}
\frac{\partial v_{1}}{\partial t}=\Delta v_{1}-2 u+v_{1}, \quad x \in \Omega, \quad t>0 \\
\frac{\partial v_{2}}{\partial t}=\Delta v_{2}-4 u-5 v_{2}, \quad x \in \Omega, \quad t>0 \\
\frac{\partial v_{1}}{\partial \nu}=\frac{\partial v_{2}}{\partial \nu}=0, \quad x \in \partial \Omega, \quad t>0 .
\end{array}\right.
$$

O exemplo acima mostra também que podemos obter sistemas de equações diferenciais parciais não-lineares em que as instabilidades dos equilíbrios parciais não implicam a instabilidade do equilíbrio correspondente do sistema original. De fato, consideremos o seguinte sistema não-linear com estrutura anti-gradiente

$$
\left\{\begin{aligned}
\frac{\partial u}{\partial t} & =\Delta u+f(u)+2 v_{1}+4 v_{2}, x \in \Omega, t>0 \\
\frac{\partial v_{1}}{\partial t} & =\Delta v_{1}-2 u+g_{1}\left(v_{1}, v_{2}\right), \quad x \in \Omega, \quad t>0 \\
\frac{\partial v_{2}}{\partial t} & =\Delta v_{2}-4 u+g_{2}\left(v_{1}, v_{2}\right), \quad x \in \Omega, \quad t>0 \\
u_{x} & =0, \quad x=0,1, \quad t>0, \\
\left(v_{1}\right)_{x} & =\left(v_{2}\right)_{x}=0 \quad x=0,1, \quad t>0
\end{aligned}\right.
$$

onde $f: \mathbb{R} \longrightarrow \mathbb{R}, u \mapsto f(u)$, é tal que $\frac{\partial H}{\partial u}\left(u, v_{1}, v_{2}\right)=f(u)+2 v_{1}+4 v_{2}$ e $g: \mathbb{R}^{2} \longrightarrow \mathbb{R}^{2}$, $\left(v_{1}, v_{2}\right) \mapsto g\left(v_{1}, v_{2}\right)=\left(g_{1}\left(v_{1}, v_{2}\right), g_{2}\left(v_{1}, v_{2}\right)\right)$, é tal que $\frac{\partial H}{\partial\left(v_{1}, v_{2}\right)}=\left(2 u-g_{1}\left(v_{1}, v_{2}\right), 4 u-\right.$ $\left.g_{2}\left(v_{1}, v_{2}\right)\right)$ e verificam as condições seguintes: $f(0)=0, g(0,0)=(0,0), f^{\prime}(0)=1$, $\nabla g_{1}(0,0)=(1,0)$ e $\nabla g_{2}(0,0)=(0,-5)$. Pela discussão realizada no exemplo temos que $(0,0,0)$ é um equilíbrio estável do sistema dado, mas separadamente 0 e $(0,0)$ são equilíbrios instáveis dos sistemas parciais correspondentes. 


\section{Conclusões}

Neste trabalho foi realizado um estudo sobre a instabilidade de equilíbrios não-constantes para equações diferenciais parciais do tipo reação-difusão, com coeficientes de difusão variáveis, definidas em domínios convexos limitados do espaço $\mathbb{R}^{N}$. Também foram abordados os sistemas de equações diferenciais parciais com acoplamento do tipo antigradiente para os quais foram admitidas condições e hipóteses no sentido de obter resultados análogos aos obtidos para as equações.

Foram obtidos alguns resultados que mostram a influência da convexidade do domínio sobre a instabilidade de equilíbrios não-constantes para equações com coeficientes variáveis, análogos aos resultados já bem conhecidos para o caso das equações com coeficientes constantes. Além disso, para $N \geq 2$, foram estabelecidas algumas condições sobre os coeficientes de difusividade que generalizam as condições obtidas em Yanagida [42] para o caso da equação escalar com coeficiente de difusão variável.

Para a verificação da infuência da propriedade geométrica do domínio sobre o equilíbrio não-constante da equação, foi obtida uma relação geométrica válida para toda função $\phi$ definida num domínio limitado $\Omega$, com derivada co-normal nula no bordo $\partial \Omega$, o Teorema 1.2.1, mediante a qual foi possível estabelecer parte da análise dos sinais dos autovalores principais dos operadores lineares associados. A relação obtida generaliza, para o caso dos coeficientes variáveis, um resultado provado em Matano [33] e em Payne [36] para o caso das equações com coeficientes constantes. Verificou-se assim, que a influência da convexidade do domínio $\Omega$ sobre a condição de instabilidade de equilíbrios nãoconstantes do problema de Neumann homogêneo para uma equação diferncial parcial dada na forma de divergência permanece verdadeira, embora seja necessário, como era de se esperar, o estabelecimento de algumas relações entre os coeficientes não-constantes da equação dada e as condições do bordo do domínio convexo. Como aplicação desse 
resultado geométrico foram obtidos, como corolários, os resultados de Casten e Holland [7] e Matano [33] sobre a instabilidade de equilíbrios não-constantes para equações de coeficientes constantes definidas em domínios convexos.

O estudo sobre a estabilidade ou a instabilidade dos equilíbrios das equações foi feito por meio da estabilidade ou da instabilidade linear e foi usado um método mini-max para avaliação dos autovalores principais dos operadores lineares associados aos equilíbrios dos problemas correspondentes dados. Com o uso do método mini-max apareceu qual deveria ser a relação entre os coeficientes de difusividade para a determinação da instabilidade dos equilíbrios não-constantes, tanto no caso das equações quanto no caso dos sistemas anti-gradientes. Por exemplo, para $N=2$ com a matriz de difusividade dada por

$$
D(x, y)=\operatorname{diag}\left\{a^{2}(x, y), b^{2}(x, y)\right\}
$$

para a garantia da instabilidade de equilíbrios não-constantes dos problemas definidos em domínios convexos é necessário exigir que os coeficientes $a$ e $b$ satisfaçam as condições seguintes:

(-) $D \Delta a=a^{2} a_{x x}+b^{2} a_{y y} \leq 0$ e $D \Delta b=a^{2} b_{x x}+b^{2} b_{y y} \leq 0$ em $\Omega$

$(-)\langle D \nabla a, \vec{n}\rangle \geq 0$ e $\langle D \nabla b, \vec{n}\rangle \geq 0$ em $\partial \Omega$;

$(-)\langle\mathbb{D}(x, y) \xi, \xi\rangle \leq 0$ para todo $(x, y) \in \Omega$ e todo $\xi \in \mathbb{R}^{2}$ onde a forma quadrática é definida pela matriz $\mathbb{D}(x, y)$ dada em (1.28).

Foram exibidos exemplos de equações com difusividades variáveis definidas em domínios convexos particulares para as quais vale a propriedade citada.

Entretanto, para as equações com coeficientes de difusividade variáveis dados na forma $k(x) I_{N}$ onde $I_{N}$ é a matriz identidade de ordem $N$, não foi possível afirmar um resultado sobre a influência da convexidade sobre a instabilidade de equilíbrio nãoconstante, com hipóteses análogas às admitidas para as equações com coeficientes de difusividade expressos por uma matriz diagonal $D(x)$.

No caso dos sistemas de equações escalares, isto é, dado quando $m=n=1$, além de relações análogas às das equações, a relação $f_{v} A+g_{u} B=0$ obtida particularizou uma classe de sistemas anti-gradientes para os quais é possível adaptar as conclusões sobre instabilidade de equilíbrios não-constantes provados para as equações. Para os sistemas de equações com coeficientes variáveis com acoplamento do tipo anti-gradiente foi obtida, portanto, uma generalização dos resultados de Yanagida [43]. Como continuação natural 
do trabalho realizado resta, entretanto, desenvolver as análises para casos de sistemas onde $m>1$ e $n>1$.

Uma questão que naturalmente se coloca é sobre a influência do método mini-max nos resultados obtidos. Em outras palavras é de se perguntar o quanto as conclusões obtidas estariam dependendo do método utilizado. Uma proposta para trabalhos futuros seria, portanto, a utilização de métodos de comparação, como utilizado em Kishimoto e Weinberger [28], por exemplo, para verificar quais das condições obtidas pelo método da linearização ainda permanecem válidas.

Uma outra direção de investigação seria o uso da ferramenta dada pela $\Gamma$-convergência como recurso talvez mais adequado para se lidar com esse tipo particular de problema, conforme sugerido por Nascimento em "Stable stationary solutions induced by spatial inhomogeneity via $\Gamma$-convergence". Bol. Soc. Bras. Mat. Vol. 29, N. 1, (1998), 75-97. 


\section{Referências Bibliográficas}

[1] R. A. Adams, Sobolev Spaces, Academic Press, NY, 1975.

[2] S. Agmon, A. Douglis and L. Niremberg, "Estimates near the boundary for solutions of elliptic partial differential equations satisfying general boundary conditions", Comm. Pure Appl. Math., 12 (1959), 623-727.

[3] N. I. Akhiezer and I. M. Glazman, Theory of Linear Operators in Hilbert Space, vol. I, II, Dover Publ., Inc., NY, 1993.

[4] P. W. Bates and K. J. Brown, "Convergence to equilibrium in a reaction-diffusion system", Nonlinear Analysis, vol 8, n. 3 (1984), 227-235.

[5] H. Brezis, Analyse Fonctionnelle: Théorie et applications, $2^{e}$ tirage, Masson, Paris, 1987.

[6] A. N. Carvalho and J. G. Ruas-Filho, "Attractors in fractional power spaces", SIAM J. Math. Anal., vol 26 no.2 (1995), 415-427.

[7] R. G. Casten and C. J. Holland, "Instability results for reaction diffusion equations with Neumann boundary conditions", J. Differential Equations, 27 (1978), 266273.

[8] N. Chafee, "Asymptotic behavior for solutions of a one-dimensional parabolic equation with homogeneous Neumann boundary conditions", J. Differential Equations, 18 (1975), 111-134.

[9] M. Chipot and J. K. Hale, "Stable equilibria with variable diffusion", Contemp. Math. 17 (J. A. Smoller, ed.), Amer. Amth. Soc., Providence, R.I., (1983), 209213. 
[10] C. Conley and J. Smoller, "Bifurcation and stability of stationary solutions of the Fitz-Hugh-Nagumo equations", J. Differential Equations, 63 (1986), 389-405.

[11] E. Conley, D. Hoff and J. Smoller, "Large time behavior of solutions of system of nonlinear reaction-diffusion equations", SIAM J. Appl. Math., vol 35, no. 1, (1978), 1-16.

[12] J. Crema, A. S. do Nascimento, "On the role of the equal-area condition in internal layer stationary solutions to a class of reaction-diffusion systems", EJDE, vol 2004 (2004), no. 99, 1-13.

[13] Y. Du and S. B. Hsu, "A difusive predator-prey model in heterogeneous environment," J. Differential Equations 203 (2004), 331-364.

[14] C. Dunford and J. T. Schwartz, Linear Operators. Part II: Spectral Theory, Self Adjoint Operators in Hilbert Space, Interscience, NY, 1958.

[15] W. H. Fleming, "A selection-migration model in population genetics ," J. Math. Biol. 2, (1975), 219-234.

[16] A. Friedman, Partial Differential Equations, Holt, Reinhart and Winstom, Inc. NY, 1969.

[17] A. Friedman, Partial Differential Equations of Parabolic Type, Prentice-Hall, Englewood Cliffs, N.J., 1964.

[18] G. B. Folland, Introduction to Partial Differential Equations, 2nd. ed., Academic Press, NJ, 1995.

[19] G. Fusco and J. K. Hale, "Stable equilibria in a scalar parabolic equation with variable diffusion", SIAM J. Math. Anal. vol 16, n. 6 (1985), 1152-1164.

[20] A. Giglioli, Equações Diferencias Parciais Elípticas, $10^{0}$ Colóquio Brasileiro de Matemática, IMPA-CNPq, 1975.

[21] D. Gilbarg and N. S. Trudinger, Elliptic Partial Differerential Equations of Second Order, Classics in Mathematics, Springer-Verlag, Berlin-Heidelberg, 2001. 
[22] J. K. Hale, Asymptotic Behavior of Dissipative Systems, Mathematical Surveys and Monographs, No. 25, Providence: American Mathematical Society, 1988.

[23] D. Henry, Geometric Theory of Semilinear Parabolic Equations, Lecture Notes in Math., vol 840, Springer-Verlag, Berlin/NY, 1981.

[24] S. Jimbo and Y. Morita, "Stability of nonconstant steady-state solutions to a Ginzburg-Landau equation in higher space dimensions," Nonlinear Analysis 22 (1984), 753-770.

[25] T. Kato, Perturbation Theory for Linear Operators, Springer-Verlag, Berlin, 1966.

[26] H. Kielhöfer, "Stability and semilinear evolution equations in Hilbert space", Arch. Rational Mech. Anal. 57 (1974), 150-165.

[27] K. Kishimoto, "Instability of non-constant equilibrium solutions of a system of competition-diffusion equations", J. Math. Biol. 13 (1981), 105-114.

[28] K. Kishimoto and H. F. Weinberger, "The spatial homogeneity of stable equilibria of some reaction-diffusion systems on convex domains", J. Differential Equations, 58 (1985), 15-21.

[29] M. G. Krein and M. A. Rutman, "Linear operators which leave a cone in Banach space invariant", Amer. Math. Soc. Trans. Series 1, n. 26, (1950). (translated from Uspekhi Mat. (N.S.) 3 (1948), 3-95).

[30] P. L. Lions, "Structure of the set of steady-state solutions and asymptotic behaviour of semilinear heat equations", J. Differential Equations, 53 (1984), 362386.

[31] O. Lopes, "Radial and nonradial minimizers for some radially symmetric functionals", Electronic Journal of Differential Equations 1996 no.3 (1996), 1-14.

[32] K. Maginu, "Stability of stationary solutions of a semilinear parabolic partial differential equation", J. Math. Anal. Appl., 63 (1978), 224-243.

[33] H. Matano, "Asymptotic behavior and stability of solutions of semilinear diffusion equations", Publ. RIMS, Kyoto University, 15 (1979), 401-454, 
[34] Y. Morita, "Reaction-diffusion systems in nonconvex domains: Invariant manifold and reduced form", J. Dynam. Differential Equations 2 (1990), 69-115.

[35] A. S. do Nascimento, "On the role of diffusivity in some stable equilibria of a diffusion equation", J. Differential Equations 155 (1999), 231-244.

[36] L. E. Payne, "Some remarks on maximum principles," Journal D'Analyse Mathématique, vol 30 (1976),421-433.

[37] M. H. Protter and H. F. Weinberger, Maximum principles in Differential Equations, Prentice-Hall, Englewood Cliffs, N.J., 1967.

[38] F. Riesz and B Sz-Nagy, Functional Analysis, Frederick Ungar, NY, 1955.

[39] J. Smoller, Shock-Waves and Reaction-Diffusion Equations, 2nd. ed. SpringerVerlag, NY, 1994.

[40] J. Smoller and A. Wasserman, "Global bifurcation of steady-state solutions," J. Differential Equations, 39 (1981), 269-290.

[41] R. Willie, "A semilinear reaction-diffusion system of equations and large diffusion," J. Dyn. and Diff. Equations, vol. 16 no. 1, (2004), 35-63.

[42] E. Yanagida, "Stability of stationary distributions in a space-dependent population growth process," J. Math. Biology, 15 (1982), 37-50.

[43] E. Yanagida, "Mini-maximizers for reaction-diffusion systems with skew-gradient structure," J. Differential Equations, 179 (2002), 311-335.

[44] E. Yanagida, "Standing pulse solutions in reaction-diffusion systems with skewgradient structure," J. Dyn. Diff. Eqn., vol 14, No.1, (2002), 189-205.

[45] K. Yosida, Functional Analysis, Springer-Verlag, NY, 1965. 